Analyse von Kundenaktivitäten und der Bereitstellungsqualität von Büchern aus Kundensicht Mit einer Untersuchung von Kundenkommentaren zu Mietangeboten für E-Books 

JANINA WITTROCK

\section{Analyse von Kundenaktivitäten und der Bereitstellungsqualität von Büchern aus Kundensicht}

Mit einer Untersuchung von Kundenkommentaren zu

Mietangeboten für E-Books 


\section{Über den Autor}

Janina Wittrock studierte bis 2012 Buchhandel und Verlagswirtschaft an der HTWK Leipzig. Während des Studiums sammelte sie unter anderem Erfahrungen im Handels- und Direktmarketing. 2012 folgte das Master-Studium Verlags- und Handelsmanagement an der HTWK Leipzig, in dessen Rahmen das vorliegende Werk als Abschlussarbeit verfasst wurde. Seit 2016 ist Wittrock für den Kundenservice und das Handelsmarketing im St. Benno Verlag in Leipzig verantwortlich.

\section{Bibliografische Information der Deutschen Nationalbibliothek}

Die Deutsche Nationalbibliothek verzeichnet diese Publikation in der Deutschen Nationalbibliografie. Detaillierte bibliografische Daten sind im Internet unter http://dnb.de abrufbar.

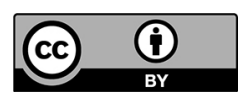

Der Text dieses Werks ist unter der Creative-Commons-Lizenz CC BY 4.0 International veröffentlicht. Den Vertragstext der Lizenz finden Sie unter https://creativecommons.org/licenses/by/4.0/. Die Abbildungen sind von dieser Lizenz ausgenommen, hier liegt das Urheberrecht beim jeweiligen Rechteinhaber.

口憏口 Die Online-Version dieser Publikation ist abrufbar unter

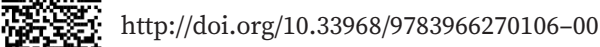

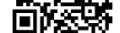

(C) 2019 Janina Wittrock

\section{Herausgeber}

Open-Access-Hochschulverlag

Hochschule für Technik, Wirtschaft und Kultur Leipzig

Karl-Liebknecht-Str. 132

04277 Leipzig

Deutschland

Druck und Bindung in Deutschland und den Niederlanden Gedruckt auf säurefreiem Papier

ISBN (Hardcover) 978-3-96627-008-3

ISBN (Softcover) 978-3-96627-009-0

ISBN (ePub) 978-3-96627-011-3

ISBN (PDF) 978-3-96627-010-6 


\section{Inhaltsverzeichnis}

Abkürzungsverzeichnis .......................................................... VII

Abbildungs- und Tabellenverzeichnis .............................................. IX

1 Einleitung .................................................................. 11

2 Systematisierung und Verbreitung von Angebotsformen für Bücher ............... 15

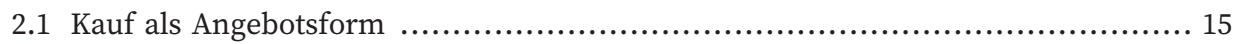

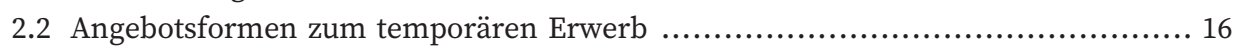

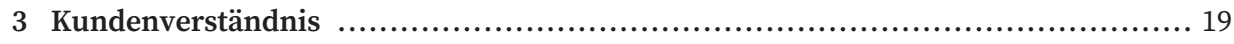

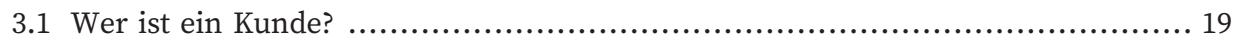

3.2 Unterschiede bei der Intensität des Bücherlesens ............................... 20

3.3 Zuwendung und Abkehr vom Bücherlesen im Lebensverlauf ...................... 21

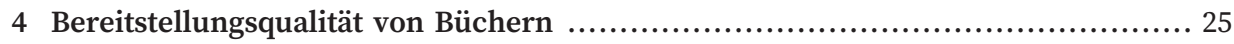

4.1 Definition der Bereitstellungsqualität ........................................ 25

4.2 Bereitstellungsdimension: Inhaltliche Vielfalt und Ordnung der Bücher ............ 27

4.3 Bereitstellungsdimension: Räumlicher und zeitlicher Zugang ...................... 32

4.3.1 Allgemeine Bereitstellungsmodi von Büchern ............................ 32

4.3.2 Bereitstellungsverfahren von Büchern bei Online-Angeboten ............... 34

4.4 Bereitstellungsdimension: Technologische Bedingungen des Zugangs .............. 36

4.4.1 Geräte zum Bücherlesen ............................................ 36

4.4.2 Softwarebasierte Benutzungsschnittstelle zum Bücherlesen ................. 39

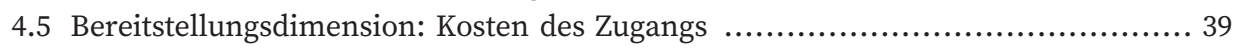

4.5.1 Finanzielle Kosten ....................................................... 39

4.5.2 Fehlende Transparenz der Benutzungsschnittstelle zum Bücherlesen ....... 43

4.5.3 Folgekosten einer Fehlentscheidung ................................... 45

4.6 Bereitstellungsdimension: Leichtigkeit der Benutzung ........................... 46

5 Kundenaktivitäten ................................................................ 49

5.1 Verständnis von Kundenaktivitäten in der aktuellen Forschung ................... 49

5.2 Verständnis von Kundenaktivitäten aus tätigkeitstheoretischer Perspektive ........ 50

5.2.1 Grundverständnis der kulturhistorischen Schule ........................ 50

5.2.2 Makrostruktur der Tätigkeit ............................................... 51

5.2.3 Tätigkeit als Austauschprozess zwischen Kunde und Umwelt ............... 53

5.3 Bedeutung des Anbieters bei den Kundenaktivitäten ............................. 54

5.4 Bedeutung von Aktivität für Kunden und Anbieter ................................ 56

5.5 Bedeutung der Umgebung für Kundenaktivität ..................................... 58

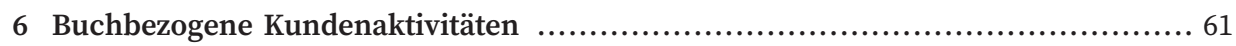

6.1 Systematisierung von buchbezogenen Kundenaktivitäten ......................... 61

6.2 Entwicklung eines Sets von buchbezogenen Kundenaktivitäten .................... 62

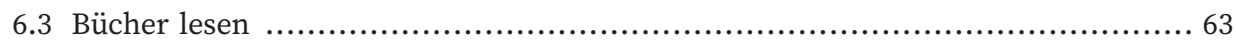

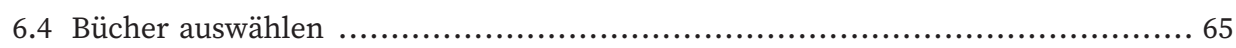

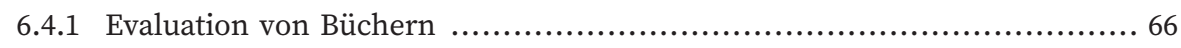




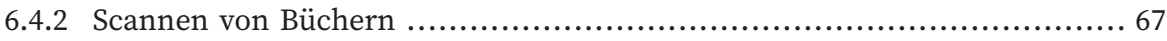

6.4.3 Verbindungen zwischen Büchern wahrnehmen .......................... 68

6.4.4 Suchanfragen für Bücher stellen .......................................... 70

6.4.5 Stöbern in einer Menge von Büchern .................................. 71

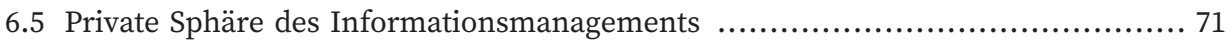

6.5.1 Bücher organisieren .................................................. 71

6.5.2 Informationen über eigene Aktivitäten organisieren ..................... 72

6.5.3 Informationen über Aktivitäten von anderen organisieren .................. 73

6.6 Öffentliche Sphäre des Informationsmanagements bei Online-Angeboten ......... 74

6.6.1 Voraussetzungen für das gemeinsame Informationsmanagement ........... 75

6.6.2 Informationen bereitstellen ohne eigene Inhalte zu erstellen ............... 76

6.6.3 Informationen bereitstellen und eigene Inhalte erstellen .................. 79

7 Inhaltsanalyse von Kundenkommentaren bei Readfy und Skoobe ................. 81

7.1 Kurzbeschreibung der untersuchten Angebote ..................................... 81

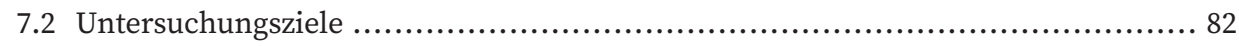

7.3 Beschreibung der Methode .................................................... 82

7.4 Festlegung des Materials und Analyse des Entstehungskontextes .................. 84

7.5 Beschreibung der Analyseeinheit und Ablauf der Untersuchung ................... 84

7.6 Maßnahmen zur Sicherstellung der Reliabilität der Ergebnisse ...................... 86

7.7 Darstellung der Ergebnisse .................................................... 88

7.8 Methodenkritik ................................................................ 102

8 Zusammenfassung der Ergebnisse und Schluss ................................ 105

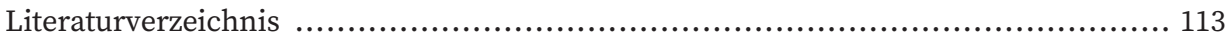

Anhang A Codeplan | „Inhaltsanalyse von Kundenkommentaren zur App von Readfy

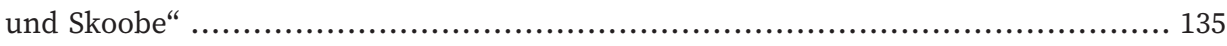

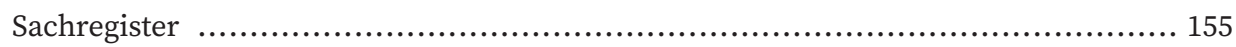




\section{Abkürzungsverzeichnis}

\begin{tabular}{|c|c|}
\hline Abb. & Abbildung \\
\hline AGB & Allgemeine Geschäftsbedingungen \\
\hline AWA & Allensbacher Markt- und Werbeträger-Analyse \\
\hline App & Application \\
\hline Aufl. & Auflage \\
\hline B2B & Business-to-Business \\
\hline BGB & Bürgerliches Gesetzbuch \\
\hline BGH & Bundesgerichtshof \\
\hline Bitkom & $\begin{array}{l}\text { Bundesverband Informationswirtschaft, Telekommunikation und neue } \\
\text { Medien }\end{array}$ \\
\hline BVMI & Bundesverband Musikindustrie \\
\hline BVT & Bundesverband Technik des Einzelhandels \\
\hline bzw. & beziehungsweise \\
\hline CEO & Chief Executive Officer \\
\hline ca. & cirka \\
\hline d. h. & das heißt \\
\hline ebd. & ebenda \\
\hline E-Book & Electronic Book \\
\hline E-Commerce & Electronic Commerce \\
\hline E-Mail & Electronic Mail \\
\hline E-Reader & Electronic Reader \\
\hline et al. & und andere \\
\hline etc. & et cetera \\
\hline f. & folgende Seite \\
\hline ff. & folgende Seiten \\
\hline GfK & Gesellschaft für Konsumforschung \\
\hline gfu & Gesellschaft für Unterhaltungs-und Kommunikationselektronik \\
\hline Hrsg. & Herausgeber \\
\hline Jg. & Jahrgang \\
\hline IfH & Institut für Handelsforschung \\
\hline Kap. & Kapitel \\
\hline LpA & Leser pro Ausgabe \\
\hline mind. & mindestens \\
\hline $\mathrm{n}$ & Stichprobenumfang \\
\hline o. J. & ohne Jahr \\
\hline PC & Personal Computer \\
\hline PDF & Portable Document Format \\
\hline Tab. & Tabelle \\
\hline u. a. & unter anderem \\
\hline
\end{tabular}


VIII Abkürzungsverzeichnis

$\begin{array}{ll}\text { USB } & \text { Universal Serial Bus } \\ \text { v. a. } & \text { vor allem } \\ \text { Vgl. } & \text { Vergleiche } \\ \text { z. B. } & \text { zum Beispiel } \\ \text { z. T. } & \text { zum Teil }\end{array}$




\section{Abbildungs- und Tabellenverzeichnis}

Tab. 3.1 Lesertypen in der Bevölkerung nach Leseintensität ......................... 20

Tab. 7.1 Reliabilitätswerte für einzelne Kategorien .............................. 86

Tab. 7.2 Übersicht der Qualitätsmerkmale bei der Bereitstellung von Büchern ....... 88

Tab. 7.3 Merkmale der Bereitstellungsdimension „Inhaltliche Vielfalt \& Ordnung der Bücher" .............................................................. 90

Tab. 7.4 Übersicht der buchbezogenen Kundenaktivitäten ............................ 96

Abb. 4.1 Dimensionen der Bereitstellungsqualität von Büchern ...................... 27

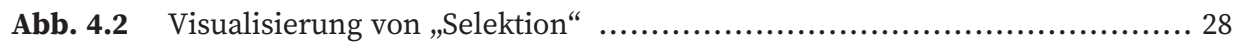

Abb. 4.3 Visualisierung von ,Zusammenfassung“ ................................... 28

Abb. 4.4 Bucharten nach Leserreichweite ............................................. 30

Abb. 4.5 Visualisierung der Bereitstellungsmodi .................................. 33

Abb. 4.6 Wesentliche Formen der Arbeitsaufteilung zwischen Server und Client ..... 35

Abb. 4.7 Entwicklung der Absatzzahlen verschiedener Gerätetypen

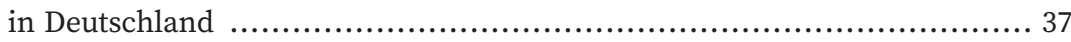

Abb. 4.8 Ausstattung deutscher Haushalte mit verschiedenen Gerätetypen ........... 38

Abb. 5.1 Makrostruktur der Tätigkeit ................................................ 52

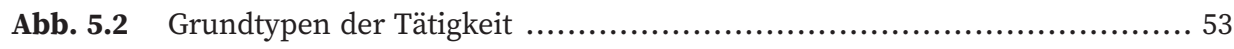

Abb. 5.3 Position und Navigation im Buch unterstützt durch Software ................ 59

Abb. 6.1 Aktivitäten eines Skripts für den Bucheinkauf im Laden ..................... 62

Abb. 6.2 Customization durch Festlegung von Themenpräferenzen ................... 67

Abb. 6.3 Visualisierung der Suchstrategie „Verbindungen folgen“ .................... 68

Abb. 6.4 Personalisierung durch algorithmisch erzeugte Empfehlungen von Kundenprofilen ..................................................... 69

Abb. 6.5 Tagcloud als Navigationswerkzeug ..................................... 70

Abb. 6.6 Rangfolge von Aktivitäten nach Einflussstärke auf das Gefühl der Agency .. 74

Abb. 6.7 Customization durch Privatsphäre-Einstellungen .......................... 76

Abb. 7.1 Beispiele für Werbeformen in einer softwarebasierten Benutzungsschnittstelle ............................................ 81

Abb. 7.2 Merkmale der Bereitstellungsdimension „Basistechnologie“................. 89

Abb. 7.3 Wahrgenommene Übereinstimmung des Bestands mit persönlichen Präferenzen .............................................................. 91

Abb. 7.4 Merkmale der wahrgenommenen Freiheit beim Bücherlesen ................. 93

Abb. 7.5 Optimierungspotenziale von Aktivitäten aus Kundensicht .................... 98 



\section{Einleitung}

Infolge der digitalisierten Bereitstellung von Medienprodukten ergibt sich für Anbieter die Chance, alternative Formen des Konsums neben dem Kauf zu etablieren. Die Akzeptanz neuer Angebotsformen spiegelt sich beispielsweise in der schnell wachsenden Kundenreichweite von Musik-On-Demand-Streaming wider. Insbesondere in der Altersgruppe 20-29 Jahre konsumieren bereits $28 \%$ (kostenlose Nutzung) bzw. 33 \% (kostenpflichtige Nutzung) der deutschen Bevölkerung Musik auf diese Weise ${ }^{1}$ Auch E-Books werden mittlerweile nicht nur zum Kauf angeboten, sondern es besteht zusätzlich die Möglichkeit, temporär auf diese zuzugreifen.

Um die damit einhergehenden Veränderungen nachvollziehen zu können, widmet sich die Forschung u. a. der Frage, welche Veränderungen sich durch neue Geschäftsmodelle für die jeweilige Branche ergeben und welche Faktoren dazu führen, dass Personen die neuen Angebote nutzen. ${ }^{2}$ Ein anderes Forschungsgebiet geht der Frage nach, warum und unter welchen Bedingungen Personen Medien in digitaler statt in materieller Form konsumieren. ${ }^{3}$

Das Forschungsinteresse dieser Arbeit besteht hingegen darin, herauszufinden, ob sich Merkmale und Bedingungen aufdecken lassen, die für Buchleser unabhängig von einer bestimmten Angebotsform wichtig sind, wenn sie Bücher erwerben.

1. Forschungsleitende Frage: Gibt es Qualitätsmerkmale bei der Bereitstellung von Büchern, die grundsätzlich wichtig für Buchleser sind?

Daneben geht es in der Arbeit einerseits um die Frage, welche Aktivitäten Leser rund um das Buch ausüben - unabhängig vom technischen Format des Buches (gedruckt/ elektronisch). Zudem soll untersucht werden, welchen buchbezogenen Aktivitäten Leser speziell in Online-Angeboten nachgehen können.

2. Forschungsleitende Frage: Welche Aktivitäten haben sich im Alltag von Buchlesern etabliert und welche buchbezogenen Aktivitäten können speziell in Online-Angeboten ausgeübt werden?

\footnotetext{
1 BVMI (2016), S. 33.

${ }^{2}$ Vgl. z. B. für Musik Dörr (2012): Music as a Service. Ein neues Geschäftsmodell für digitale Musik, Berlin: epubli; vgl. z. B. für Bücher Lulei (2015): E-Books kaufen, abonnieren, leihen? Eine Analyse auf Basis einer Konsumentenbefragung, in: Initialen. Mainzer Institut für Buchwissenschaft; vgl. Tesar (2013).

${ }^{3}$ Vgl. z. B. für Musik Hartmann et al. (2007): Nutzen und Kosten von Online-Optionen der Musikbeschaffung, in: M\&K. Sonderband 1 „Musik und Medien“, S. 105 -119; vgl. z. B. für Bücher Gerlach (2014).
} 
Aufgrund der zwei Themenkomplexe gliedert sich die Arbeit in zwei Hauptteile: In Kapitel 4 wird das Konzept der Bereitstellungsqualität eingeführt und die einzelnen Qualitätsmerkmale, die aus Kundensicht bei der Bereitstellung von Büchern eine Rolle spielen können, erläutert. In Kapitel 5 und 6 geht es um die Kundenaktivitäten und ihre Bedeutung sowohl für den Kunden als auch für den Anbieter. In den zwei vorangehenden Kapiteln werden die Angebotsformen für Bücher auf dem Markt systematisiert (Kauf, Leihe, Miete) (Kap. 2) sowie das Kundenverständnis definiert und die Bevölkerung nach ihrer Leseaktivität typisiert (Kap. 3).

Der Arbeit liegt in Anlehnung an die Tätigkeitstheorie die Annahme zugrunde, dass Angebote für Kunden Mittel sind, die ihnen helfen ihre Ziele zu erreichen bzw. ihren Interessen nachzugehen. Indem Kunden die Angebote instrumentell einsetzen, um z. B. Bücher zu suchen und zu lesen oder um Objekte zu erzeugen und zu verändern, können sie ihr Verhalten selbstbestimmt kontrollieren. ${ }^{4}$ In welchem Ausmaß sie dies tun können, hängt entschieden von der Angebotsgestaltung ab. Die leitende Frage aus Anbietersicht ist: Wie kann das Angebot gestalten werden, sodass Kunden bereit sind, dafür zu bezahlen bzw. es überhaupt zu nutzen? ${ }^{5}$ Die Bezugnahme auf die Selbstbestimmung von Kunden deutet bereits an, dass Anbieter bei der Beantwortung dieser Frage nicht umhinkommen, die Lebenswirklichkeit von Buchlesern einzubeziehen, um ein Angebot zu schaffen, auf dass Kunden im Alltag gerne zurückgreifen. Insbesondere bei E-Books werden Anbieter von Lesegeräten und -software direkt mit dem Nutzungsverhalten ihrer Kunden konfrontiert, was die Herausforderung mit sich bringt zu verstehen, was Kunden angenehm und nützlich finden, wenn sie mit Büchern umgehen:

„Im E-Book-Bereich sind wir Händler nicht mehr nur dafür verantwortlich, dass Kunden das passende Buch finden und kaufen können, sondern für das gesamte Leseerlebnis: die Präsentation des Buches, die Interaktion des Lesers mit dem Buch, das Umblättern der Seiten, die Optik. Das bringt uns dazu, dass wir darüber nachdenken, was es bedeutet, ein Leser zu sein. Wir machen uns Gedanken darüber, wie wir den gesamten Lese-Prozess interessanter und angenehmer gestalten können. Es geht um mehr als nur den technischen Wandel von Print zu Digital. "6 (Tamblyn (CEO Rakuten Kobo)

Tatsächlich ist über die Art und Weise, wie Bücher z. B. gelesen werden, jedoch wenig bekannt:

„Was uns aber fehlt (...) ist, dass wir tatsächlich zur Nutzung von gedruckten Büchern und wie intensiv und wie konzentriert und wie vollständig Menschen das eigentlich gelesen haben (...) eigentlich überhaupt gar keine gute Empirie haben. Das heißt, wir referenzieren jetzt immer auf so Wunschvorstellungen zurück wie das wohl im Print gewesen sein könnte oder nach unseren Vorstellungen gewesen zu sein hat-

\footnotetext{
${ }^{4}$ Engeström (2008), S. 33.

${ }^{5}$ Vgl. Heinonen/ Strandvik (2015), S. 476.

${ }^{6}$ Kivelip (2011), buchreport.de.
} 
wir wissen aber nicht, ob das so war. "7 (Hagenhoff (Professorin am Institut für Buchwissenschaften in Erlangen))

Daraus ergibt sich die Fragestellung, welche Funktionen für Buchleser konkret nützlich sind.

3. Forschungsleitende Frage: Mit welchen Funktionen können Anbieter von Büchern ihre Kunden bei buchbezogenen Aktivitäten unterstützen?

Mithilfe einer Inhaltsanalyse werden die in den Kapiteln 3 bis 6 gewonnenen Erkenntnisse am Ende der Arbeit (Kap. 7) dahingehend geprüft, ob diese die Sichtweise von Kunden und ihre Aktivität widerspiegeln. Als Untersuchungsmaterial werden Kundenkommentare zu zwei Vermietungsangeboten für E-Books herangezogen. Kapitel 8 fasst die Untersuchungsergebnisse zusammen, leitet Gestaltungsempfehlungen für Anbieter ab und benennt offene gebliebene Fragen.

\footnotetext{
${ }^{7}$ Vgl. Siegemund (2016), ab Min. 6.40 Min., https://detektor.fm/kultur/netflixierung-des-buches.
} 



\section{Systematisierung und Verbreitung von Angebotsformen für Bücher}

In der Realität existieren zwei Nutzungsformen von Objekten, die sich darin unterscheiden, dass eine Person entweder dauerhaft über ein Objekt verfügen kann oder nur temporär. ${ }^{8}$ Zieht man diese Unterscheidung zur Typisierung von Angeboten heran, lässt sich auf dem Buchmarkt das Kaufmodell vom Leih- und vom Mietmodell abgrenzen, bei denen Bücher nur temporär genutzt werden können. ${ }^{9}$ Aus rechtlicher Sicht kommen Tausch und Schenkung als Erwerbstypen hinzu. ${ }^{10}$ Daneben können Bücher mit Freunden/Bekannten geteilt ${ }^{11}$ oder Kopien erstellt werden. ${ }^{12}$ Nachfolgend werden jedoch nur der Kauf, die Leihe und die Miete näher betrachtet.

\subsection{Kauf als Angebotsform}

Juristisch kann der dauerhafte Erwerb von E-Books nicht mit dem Kauf im Sinne des BGB bei gedruckten Büchern gleichgesetzt werden, da häufig kein Eigentum erworben wird und/oder die Verträge zeitlich befristet bzw. kündbar sind. ${ }^{13}$ Trotzdem ist die Bezeichnung Kauf üblich.

Die Käuferreichweite bei gedruckten Büchern nimmt kaum merklich, aber kontinuierlich ab: im Jahr 2013 lag sie bei 59,1 \%, ein Jahr später bei 59 \%, im Jahr 2015 bei 58,3 \% und im Jahr 2016 bei 58,1 \%. ${ }^{14}$ Die Käuferreichweite bei E-Books hingegen steigt. Insgesamt lässt sich ein Wachstum um 2,3 \% seit dem Jahr 2013 auf aktuell 8,3 $\%$ (2016) feststellen. ${ }^{15}$ Es lässt sich beobachten, dass Buchleser zunehmend auf Alternativen zum Kauf zurückgreifen, sodass mittlerweile $40 \%$ (2015) von ihnen keine Bücher kaufen. ${ }^{16}$ Seit 2005 hat sich der Anteil der selbst gekauften an den gelesenen Büchern um $15 \%$ verringert. Der Rückgang betrifft fast nur neue Bücher, da der Gebrauchtkaufanteil stabil bei rund $18 \%$ liegt. ${ }^{17}$ Den größten Anteil am Gesamtumsatz (Bruttoverkaufspreis) aller buchhändlerischen Betriebe haben Sortiments-

\footnotetext{
${ }^{8}$ Vgl. Zentes/ Freer/ Beham (2013), S. 5.

9 Vgl. Stiftung Lesen (Hrsg.) (2009), S. 166 f.; vgl. Börsenverein (Hrsg.) (2015), S. 82.

${ }^{10}$ Vgl. Weyers/ Sujbert/ Eckensberger (2007), S. 126; vgl. auch Zentes/ Freer/ Beham (2013), S. 5.

11 Vgl. Ebd.

12 Vgl. Tietzel (1995), S. 108.

${ }^{13}$ Vgl. Graef (2016), S. 35.

${ }^{14}$ Vgl. AWA (2013), S. 58; vgl. AWA (2014), S. 65; vgl. AWA (2015), S. 65; vgl. AWA (2016), S. 64.

${ }^{15}$ Vgl. AWA (2013), S. 59; vgl. AWA (2014), S. 66; vgl. AWA (2015), S. 66; vgl. AWA (2016), S. 65.

16 Vgl. Börsenverein (Hrsg.) (2015), S. $107 \mathrm{f}$.

17 Vgl. Börsenverein (Hrsg.) (2008), S. 113; vgl. Börsenverein (Hrsg.) (2015), S. 81.
} 
buchhandel und E-Commerce. ${ }^{18}$ Daneben zeichnet sich auch ein gesellschaftlicher Trend der temporären Nutzung ab, der u. a. durch ökologische Motive erklärt wird. ${ }^{19}$ Der Trend betrifft sowohl materielle als auch digitale Produkte. ${ }^{20}$ Das Phänomen wird auch als Ausdruck einer Anti-Konsum-Einstellung untersucht, die dazu führt, dass das Teilen von Ressourcen bevorzugt wird. Die Einstellung weist eine soziale Sphäre, z. B. Umweltschutz, und/oder eine persönliche Sphäre, z. B. Lebensvereinfachung, auf. ${ }^{21}$

\subsection{Angebotsformen zum temporären Erwerb}

Auch beim temporären Erwerb ist bei E-Books die juristisch korrekte Begriffsverwendung nicht eindeutig geklärt. Dies hat zwei Gründe: Zum einen wird von Verleih gesprochen, wenn es aus rechtlicher Sicht um Vermietung geht. Bitkom grenzt die EBook-Ausleihe bei kommerziellen Anbietern von der E-Book-Ausleihe in öffentlichen Bibliotheken $a b .^{22}$ Damit orientieren sich die Marktforscher am alltäglichen Sprachgebrauch, da z. B. auch in Videotheken Filme ,ausgeliehen“ werden. ${ }^{23}$ Zum anderen ist analog zum Kauf keine einfache Übertragung der Begriffe „Leihe“ und „Vermietung“ auf E-Books möglich. Da E-Books keine Sachen im Sinne des BGB sind, handelt es sich um Lizenzverträge oder mietähnliche Verträge. ${ }^{24}$ Gemäß dieser haben Kunden einen schuldrechtlichen Anspruch auf die Möglichkeit des Werkgenusses per Cloud-Computing ${ }^{25}$ und je nach Vertrag auf die Möglichkeit, ein Buch offline zu lesen. ${ }^{26}$

Beim Begriff „Leihe“ ist problematisch, dass bei E-Books kein Besitzerwechsel und keine Rückgabe stattfinden. ${ }^{27}$ Graef hält den Begriff trotzdem für geeignet, wenn die Zugangsberechtigung nur temporär besteht und die Datei nach Ablauf der Leihfrist unbrauchbar gemacht wird. ${ }^{28}$ Das Leihmodell für E-Books wird in dieser Form von den öffentlichen Bibliotheken in Kooperation mit Dienstleistern umge-

\footnotetext{
${ }^{18}$ Vgl. Börsenverein (Hrsg.) (2015), S. 9; Beim Direktvertrieb von Verlagen sind die Angebotsformen unklar.

19 Vgl. Zentes/ Freer/ Beham (2013), S. 4.

20 Vgl. ebd.

${ }^{21}$ Vgl. Iyer/ Munci (2009), S. $160 \mathrm{f}$.

22 Vgl. Bitkom (2015), S. 11.

${ }^{23}$ Vgl. Loest (1984), S. 61.

${ }^{24}$ Vgl. Graef (2016), S. 32, S. 36; vgl. Zech (2014).

${ }^{25}$ Vgl. Zech (2014).

${ }^{26}$ Ob dem Benutzer das Nutzungsrecht der Vervielfältigung eingeräumt werden muss, ist rechtlich nicht geklärt (vgl. Zech (2014)). Generell wird zwischen der Nutzung im Sinn einer urheberrechtlich relevanten Handlung und dem Werkgenuss, der urheberrechtlich ohne Bedeutung ist, unterschieden (Juraschko (2015), S. 6; Vgl. Zech (2014)).

27 Vgl. Graef (2016), S. 46 f.

${ }^{28}$ Vgl. Graef (2016), S. 47.
} 
setzt $^{29}$, da das Angebot nicht-kommerziell und der Leihvorgang selbst kostenlos ist. $^{30}{ }^{31}$ Insgesamt sind derzeit $26 \%$ der Bevölkerung ein aktives Mitglied bei einer öffentlichen Bibliothek. ${ }^{32}$ Die Möglichkeit der E-Book-Leihe nutzen 4 \% der Bevölkerung. ${ }^{33}$ Betrachtet man ausschließlich das Verhalten von E-Book-Lesern, ergibt sich seit 2013 eine Steigerung der E-Book-Leihe um $15 \%$ auf $32 \%$ im Jahr $2015 .{ }^{34} \mathrm{Da}$ die Anzahl an teilnehmenden Bibliotheken bzw. Verbünden und Kunden kontinuierlich wächst, führt dies zu mehr Ausleihen. Ihre Anzahl ist seit $2013 \mathrm{um}$ ca. 9,5 Millionen auf ca. 17,5 Millionen im Jahr 2015 angestiegen (Hörbücher und Bücher). ${ }^{35}$ Zudem können Bibliotheken mittlerweile E-Books von marktführenden Publikumsverlagen lizenzieren. ${ }^{36}$ Die Entwicklung lässt Verlage vermuten, dass ein Zusammenhang zum eher stagnierenden Umsatz und Absatz auf dem Käufermarkt besteht. $^{37}$

Beim Mietmodell gewährleistet der Anbieter eine zeitlich befristete Zugangsberechtigung zu den Büchern. ${ }^{38}$ Das Erlösmodell spielt für die Definition keine Rolle, sondern es kommt darauf an, dass die Gebrauchsüberlassung Erwerbszwecken dient. ${ }^{39}$ Jedoch besteht das Problem, dass dem Benutzer kein Besitz verschafft wird, sondern nur die Zugangsmöglichkeit. ${ }^{40}$ Nach Graef könnte mit Blick auf ein Urteil des BGH zu Computerprogrammen jedoch die Ansicht vertreten werden, dass die Besitzverschaffung nicht nötig ist, sondern die Abrufoption über das Internet ausreicht. ${ }^{41}$ Bei gedruckten Büchern ist die Miete als historische Angebotsform einzustufen, da sich das Aussterben des Gewerbes der Leihbüchereien bereits Ende der 1960er Jahre abzeichnete. ${ }^{42}$ Trotzdem bietet z. B. das Angebot „CampusRitter“ die Möglichkeit, Fachbücher zu mieten. ${ }^{43}$ Als Herausforderung betrachten die Betreiber die Situation, dass in Deutschland bei gedruckten Büchern das Mietmodell mental nicht mehr repräsentiert ist: „Dieser Markt ist bisher nicht entwickelt.“44 (Thiel, Mitbegründer von „CampusRitter“). Bei E-Books gewinnt die Miete wieder an Be-

\footnotetext{
${ }^{29}$ In den meisten Fällen ist der Dienstleister die divibib GmbH mit dem Angebot „Onleihe“.

${ }^{30}$ Vgl. Graef (2016), S. $48 \mathrm{f}$.

${ }^{31}$ Alternativ können auch gemeinschaftlich, nicht öffentlich finanzierte Leihangebote entwickelt werden. Ein Beispiel hierfür sind Toy librarys in Neuseeland (vgl. Ozanne/Ballantine (2010), S. 486)

${ }^{32}$ Institut für Demoskopie Allensbach (Hrsg.) (2016), S. 2; Vorgabe: „Bibliothek in den letzten 12 Monaten genutzt."

${ }^{33}$ Vgl. Börsenverein (Hrsg.) (2015), S. $81 \mathrm{f}$.

${ }^{34}$ Vgl. Bitkom (2013), S. 11; vgl. Bitkom (2014), S. 9; vgl. Bitkom (2015), S. 10.

${ }^{35}$ Vgl. Nutzen oder Schaden? (2016), buchreport.de; vgl. Verkauf vs. Onleihe (2015)/ Buchreport, S. 8.

${ }^{36}$ Vgl. Bonnier gibt E-Books für Onleihe frei (2016), buchreport.de.

37 Vgl. Verkauf vs. Onleihe (2015), S. 8.

${ }^{38}$ Vgl. Graef (2016), S. 39.

${ }^{39}$ Vgl. Graef (2016), S. 41 f., S. 48.

${ }^{40}$ Vgl. Zech (2014).

${ }^{41}$ Vgl. Graef (2016), S. 40 f.

${ }^{42}$ Vgl. Arnim/ Knilli (1966), S. 132.

${ }^{43}$ https://www.campusritter.de

${ }^{44}$ Vgl. Horch (2014).
} 
deutung. Derzeit mieten $3 \%$ der Bevölkerung E-Books. ${ }^{45}$ Im Zeitverlauf hat sich die Nutzungsintensität der Miete bei E-Book-Lesern jedoch nicht so stark erhöht wie bei der Leihe. Aktuell nutzen $19 \%$ von ihnen Mietmodelle, was einer Steigerung von $4 \%$ zum Vergleichsjahr 2013 entspricht. ${ }^{46}$ Sowohl bei der Leihe als auch bei der Miete kann die Nutzungsmöglichkeit auf einen Einzelabruf beschränkt oder durch ein Dauerschuldverhältnis in einem vereinbarten Zeitraum geregelt sein.

Der empirische Teil der Arbeit untersucht Kundenkommentare zu den beiden deutschen E-Book-Vermietungsangeboten „Readfy“ und „Skoobe“.

${ }^{45}$ Vgl. Börsenverein (Hrsg.) (2015), S. $81 \mathrm{f}$.

${ }^{46}$ Vgl. Bitkom (2013), S. 11; vgl. Bitkom (2014), S. 9; vgl. Bitkom (2015), S. 10. 


\section{Kundenverständnis}

\subsection{Wer ist ein Kunde?}

Kunden in dieser Arbeit sind diejenigen Personen, die ein Angebot nutzen oder genutzt haben. ${ }^{47}$ Durch den direkten Kontakt mit dem Angebot konnten Kunden Erfahrungen sammeln und sich ein Urteil über das Angebot bilden. Es wird angenommen, dass Kunden ein Angebot dahingehend beurteilen, in welchen Situationen die Nutzung für sie wertvoll ist. Heinonen spricht vom Value-in-use und meint damit „the positive (or sometimes negative) value that the customer experiences when using the service ${ }^{\text {“48 }}$. Der Wert ergibt sich nicht, weil bestimmte Eigenschaften vorhanden sind, sondern Wert entsteht durch die Einbettung des Angebots in die Aktivitäten des Kunden. ${ }^{49}$ Value-in-Use entsteht auch außerhalb der Angebotsnutzung, also davor und danach z. B. wenn der Kunde Vorfreude entwickelt oder sich an positive Erlebnisse erinnert. ${ }^{50}$ Ein Kunde erlebt den Wert immer in Relation zu etwas anderem, also als geringwertiger, gleichwertig oder wertvoller. ${ }^{51}$ Frühere Erfahrungen und Erlebnisse des Kunden mit dem Anbieter (Vorgeschichte) beeinflussen das Erleben ebenso wie Erlebnisse mit anderen Anbietern. ${ }^{52}$ Was der Kunde als wertvoll empfindet, wird u. a. durch die Aufgaben, denen ein Kunde Zeit widmen möchte oder muss und dem, was er dazu braucht, bestimmt. Der Wert wird durch Prozesse des Erlebens und Interpretierens hervorgerufen, die ihm nicht unbedingt bewusst sind. ${ }^{53}$

Darüber hinaus wird angenommen, dass Kunden Buchleser sind und ein allgemeines Interesse an Büchern haben. Interesse lässt sich als „längerfristiger oder dauerhafter Bezug einer Person zu einem Gegenstand oder Gegenstandsbereich“ ${ }^{\text { }}{ }^{4}$ definieren. Dabei muss unterschieden werden, ob sich das Interesse auf ein Objekt (Buch als Sammelobjekt) oder auf eine Tätigkeit richtet, sodass das Objekt nur Mittel zum Zweck ist (z. B. Bücher lesen). ${ }^{55}$ In dieser Arbeit geht es um das Tätigkeitsinteresse. Das Interesse selbst geht mit Motivation einher, die sich im Verhalten zeigt, sich Gegenständen/Situationen anzunähern und daran, dass sich eine Person in-

\footnotetext{
${ }^{47}$ Vgl. Silberer (2009), S. 17.

${ }^{48}$ Heinonen (2009), S. 3.

49 Vgl. Mickelsson (2014), S. 48.

${ }^{50}$ Vgl. Heinonen et al. (2010), S. 539.

${ }^{51}$ Vgl. Heinonen/ Strandvik/ Voima (2013), S. 111; vgl. Holbrook (1999), S. 6; vgl. Heinonen (2009), S. 8.

52 Vgl. Heinonen/ Strandvik/ Voima (2013), S. 110; vgl. Heinonen/ Strandvik (2015), S. 480.

${ }^{53}$ Vgl. Heinonen/ Strandvik (2015), S. 479; vgl. Heinonen/ Strandvik/ Voima (2013), S. 105, S. 113.

${ }^{54}$ Oerter/ Montada (2008), S. 965.

${ }^{55}$ Holodynski/ Oerter (2008), S. 541; vgl. Oerter/ Montada (2008), S. 965 f.
} 
nerlich bewegen lässt, d. h. stark betroffen ist. ${ }^{56}$ Buchleser werden im Folgenden danach typisiert, wie intensiv sie die Lesetätigkeit ausüben und welche Bedeutung das Bücherlesen in ihrem Leben hat.

\subsection{Unterschiede bei der Intensität des Bücherlesens}

Um messen zu können, wie intensiv eine Tätigkeit ausgeübt wird, ist eine Definition von „Intensität“ nötig. Da es sich um einen abstrakten Begriff mit nur indirektem empirischem Bezug zur Realität handelt, muss mindestens ein Indikator herangezogen werden, der dem Begriff seine Identität verleiht. ${ }^{57}$ Beispiele sind die gelesenen Bücher oder die Häufigkeit der Ausübung. Auf diese Weise lässt sich die Bevölkerung in Intensitätsgruppen einteilen. Börsenverein und Stiftung Lesen erfassen hierfür die jährlich gelesene Bücherzahl. ${ }^{58} \mathrm{Zum}$ anderen ermitteln Stiftung Lesen und AWA die Häufigkeit über Intervalle. Von Viellesern lässt sich sprechen, wenn täglich oder fast täglich gelesen wird (vgl. Tab. 3.1). Vergleicht man die Typeneinteilung nach Indikator, zeigt sich, dass bei Viellesern und Durchschnittslesern ähnlich hohe Anteile ermittelt werden (31\% zu $25 \%$ bzw. $28 \%$ zu $23 \%$ ), während er sich bei Weniglesern stark unterscheidet (16\% zu $43 \%$ ).

Tab. 3.1 Lesertypen in der Bevölkerung nach Leseintensität, in \%, Werte in () = BV (Quelle: SL: Stiftung Lesen (2008), S. 160, S. 164; BV: Börsenverein (Hrsg.) (2005), S. 18; Börsenverein (Hrsg.) (2008), S. 19; Börsenverein (Hrsg.) (2015), S. 54); AWA (2011), S. 61; AWA (2013), S. 58; AWA (2015), S. 65).

\begin{tabular}{|c|c|c|c|c|c|c|}
\hline Lesertyp & $\begin{array}{l}\text { Kriterium: } \\
\text { Häufigkeit }\end{array}$ & $\begin{array}{l}S L \\
2008\end{array}$ & $\begin{array}{l}\text { AWA } \\
11 / \\
13 / \\
15\end{array}$ & $\begin{array}{l}\text { Kriterium: } \\
\text { Stück pro Jahr }\end{array}$ & $\begin{array}{l}\text { SL } \\
2008\end{array}$ & $\begin{array}{l}\text { BV } \\
05 / \\
08 / \\
15\end{array}$ \\
\hline Nichtleser & I & 25 & I & kein Buch & I & $\begin{array}{l}9 / \\
10 / \\
17\end{array}$ \\
\hline \multirow[b]{2}{*}{ Wenigleser } & \multirow{2}{*}{$\begin{array}{l}\text { seltener als } 1 \mathrm{x} \text { im } \\
\text { Monat }\end{array}$} & \multirow[b]{2}{*}{16} & \multirow{2}{*}{$\begin{array}{l}35 / \\
34 / \\
38\end{array}$} & $\begin{array}{l}\text { 1- } 5 \text { Bücher } \\
6-10 \text { (9) Bücher }\end{array}$ & $\begin{array}{l}44 \\
27\end{array}$ & \\
\hline & & & & Summe & 71 & $\begin{array}{l}40 / \\
43 / \\
41\end{array}$ \\
\hline
\end{tabular}

56 Vgl. Holodynski/ Oerter (2008), S. 540.

57 Vgl. Brosius/ Haas/ Koschel (2012), S. $21 \mathrm{f}$.

58 Börsenverein: Wenigleser 1-9, Durchschnitts- bzw. Gelegenheitsleser ab 9,5 Bücher und Vielleser ab 18,5 Bücher pro Jahr/ Stiftung Lesen: Wenigleser 1-10 Bücher, Durchschnitts- bzw. Gelegenheitsleser 11 - 20 Bücher, Vielleser ab 21 Bücher (vgl. Börsenverein (Hrsg.) (2015), S. 54; vgl. Stiftung Lesen (Hrsg.) (2009), S. 164). 


\begin{tabular}{|c|c|c|c|c|c|c|}
\hline \multirow[t]{2}{*}{$\begin{array}{l}\text { Durch- } \\
\text { schnittsleser }\end{array}$} & $\begin{array}{l}\text { etwa } 1 x \text { pro } \\
\text { Woche } \\
\text { so alle } 14 \text { Tage } \\
\text { ungefähr } 1 x \\
\text { Monat }\end{array}$ & $\begin{array}{l}12 \\
8 \\
8\end{array}$ & $\begin{array}{l}10 \\
8 \\
10\end{array}$ & \multirow[t]{2}{*}{$\begin{array}{l}11-20(18,5) \\
\text { Bücher }\end{array}$} & \multirow[t]{2}{*}{17} & $\begin{array}{l}27 / \\
23 /\end{array}$ \\
\hline & Summe & 28 & $\begin{array}{l}28 / \\
28 / \\
28\end{array}$ & & & \\
\hline \multirow{2}{*}{ Vielleser } & $\begin{array}{l}\text { täglich } \\
\text { mehrmals in der } \\
\text { Woche }\end{array}$ & $\begin{array}{l}9 \\
22\end{array}$ & $\begin{array}{l}14 \\
21\end{array}$ & $\begin{array}{l}21 \text { - } 50 \text { Bücher } \\
\text { ab } 51 \text { Bücher (ab 18,5 } \\
\text { Bücher) }\end{array}$ & $\begin{array}{l}8 \\
3\end{array}$ & \\
\hline & Summe & 31 & $\begin{array}{l}37 / \\
38 / \\
35\end{array}$ & Summe & 11 & $\begin{array}{l}27 / \\
25 / \\
22\end{array}$ \\
\hline
\end{tabular}

Erschwerend kommt hinzu, dass mitunter die Antwortoption „kein Buch gelesen“ in der Befragung fehlt, sodass die Aussagekraft der Typisierung auf Basis dieser gewonnenen Daten eingeschränkt ist, aber auch die Vergleichbarkeit der Ergebnisse untereinander. Insgesamt werden mit dem Indikator „Lesehäufigkeit“ höhere Anteile bei Viel- und Durchschnittslesern gemessen, was vermutlich dadurch bedingt ist, dass ein Buch über einen längeren Zeitraum gelesen wird. Zudem ergibt sich für Befragte die Unsicherheit, ab wann ein Buch als gelesen gilt. Der Trend im Zeitverlauf ist jedoch unabhängig vom Indikator eindeutig: Während der Anteil an Nicht- und Weniglesern zunimmt, geht der Anteil an Viellesern zurück.

\subsection{Zuwendung und Abkehr vom Bücherlesen im Lebensverlauf}

Im Folgenden soll das Zusammenspiel von Einstellung und Tätigkeitsausübung näher betrachtet werden. Eine hohe Wichtigkeit bzw. subjektive Bedeutsamkeit (kognitiv) und starke Betroffenheit (affektiv) bei einer Tätigkeit deuten darauf hin, dass das Lesen von Büchern für die Identität einer Person eine Rolle spielt. Die Identität wird von der Person selbst konstruiert und definiert sich immer in Relation zu anderen Größen, z. B. „Ich als Leser heute im Vergleich zu früher“ oder „Meine Vorstellungen von mir als Leser gegenüber Vorstellungen von anderen über mich“. ${ }^{59}$ Der Grad der innerlichen Verpflichtung hat einen Einfluss darauf, ob ein Interesse im Sinne einer Gegenstandbeziehung Bestand hat oder nicht. Eine Abkehr

${ }^{59}$ Vgl. Haußer (1995), S. 2 ff., S. 7 f. 
von der Tätigkeit lässt sich als Lösung der Bindung auffassen. ${ }^{60}$ Die wahrgenommene Wichtigkeit kann ein Hinweis darauf sein, dass eine Person beabsichtigt, ein Medium zu nutzen oder dass sie es auch tatsächlich nutzt. Die Einstellung und das tatsächliche Verhalten können sich jedoch widersprechen. ${ }^{61}$ Bei einem Vergleich der Wichtigkeit eines Mediums und seiner Nutzungsfrequenz bestätigt sich, dass von der wahrgenommenen Wichtigkeit nicht automatisch auf das tatsächliche Verhalten geschlossen werden kann. Während $40 \%$ das Lesen von Fach- und Sachbüchern als wichtig bewerten, fällt die Häufigkeit der Tätigkeitsausübung mit durchschnittlich $16 \%$ deutlich geringer aus. ${ }^{62}$ Eine Ausnahme bildet die Altersgruppe $14-19$ Jahre, bei der das Verhältnis nahezu ausgeglichen ist ${ }^{63}$; vermutlich aufgrund von Schulund Ausbildungslektüre. Beim Musikhören ist die Diskrepanz deutlich geringer. Beim Fernsehen wiederum kehrt sich das Diskrepanzverhältnis sogar um. Hier üben Personen die Tätigkeit aus, obwohl sie Fernsehen als eher unwichtig bewerten. ${ }^{64}$ Eine mögliche Erklärung der Diskrepanz beim Bücherlesen ist eine Antwortverzerrung durch soziale Erwünschtheit. Die Antwort sagt in diesem Fall nichts über das aus, was sie messen soll und kann auf den Wunsch nach sozialer Anerkennung des Befragten und seinen Eindruck zurückgeführt werden, dass die Ausübung der Tätigkeit gesellschaftlich erwartet wird. ${ }^{65}$ Ein anderer Grund könnte sein, dass es nicht gelingt, eine Verhaltensabsicht im Alltag zu realisieren. In diesem Fall ist eine Person dem Bücherlesen trotzdem innerlich verbunden (affektive Komponente). Köcher bestätigt empirisch, dass die Ursache für den Verzicht auf das Bücherlesen nicht immer fehlendes Tätigkeitsinteresse ist. Sie untersucht die Lesetätigkeit im Lebensverlauf, d. h. im Mittelpunkt der Betrachtung steht nicht die Ausübung der Tätigkeit zu einem bestimmten Zeitpunkt - wie bei den Lesertypen - sondern wie sich das Verhalten einer Person und die subjektive Bedeutsamkeit der Tätigkeit im Leben entwickeln. Köcher schlägt eine Systematisierung vor, die für die deutsche Bevölkerung repräsentativ ist. Die Rückkehrer mit 6 \% Anteil (Lange nicht gelesen, jetzt wieder intensiv) sind am häufigsten in den Altersgruppen $16-29$ Jahre und über 60 Jahre anzutreffen (jeweils $34 \%$ ), während Abbrecher/ehemalige Leser mit einem Anteil von $17 \%$ (Früher intensiv gelesen, heute wenig oder nie) am häufigsten in der Altersphase zwischen $30-44$ (35\%) und $45-59$ Jahre (31\%) zu finden sind. ${ }^{66}$ Die Rückkehrer bilden die kleinste Gruppe, während über $50 \%$ der Bevölkerung entweder kein Interesse an der Tätigkeit Lesen hat (war nie von persönlicher Bedeutung) oder nur sporadisch liest (nie regelmäßig). Im Zuge von Köchers Untersuchung stellt sich heraus, dass ehemalige Leser häufig verhinderte Leser sind. ${ }^{67}$

\footnotetext{
${ }^{60}$ Vgl. Haußer (1995), S. 49 ff.

${ }^{61}$ Vgl. Mahrt (2014), S. 113.

${ }^{62}$ Vgl. Stiftung Lesen (Hrsg.) (2009), S. 146, S. 148.

${ }^{63}$ Vgl. Stiftung Lesen (Hrsg.) (2009), S. $146 \mathrm{f}$.

${ }^{64}$ Vgl. Stiftung Lesen (Hrsg.) (2009), S. 146, S. 148.

${ }^{65}$ Vgl. Schnell/ Hill/ Esser (2011), S. 348; vgl. Casimir (2008), S. 88.

${ }^{66}$ Vgl. Köcher (1993), S. 224 ff.

${ }^{67}$ Die weiteren Untersuchungsergebnisse beschränken sich auf die Altersgruppe 21 - 29 Jahre.
} 
Von diesen geben über $60 \%$ an, dass sie gerne mehr lesen würden. ${ }^{68}$ Während über die Hälfte von ihnen der Aussage zustimmt „Ich habe zu wenig Zeit für mich allein“, stimmen dieser nur $22 \%$ der kontinuierlichen Leser zu. Zudem geben nur knapp $38 \%$ der ehemaligen Leser an „Ich achte darauf, daß meine Interessen nicht zu kurz kommen", während es bei den kontinuierlichen Lesern $56 \%$ sind. ${ }^{69}$ Köcher schlussfolgert daraus, dass eine hohe Bereitschaft, sich an die Bedingungen des Lebensumfelds anzupassen, eine kontinuierliche Ausübung des Bücherlesens gefährdet, weil die Person nicht/selten den eigenen Interessen nachgeht. ${ }^{70}$ Darüber hinaus stellt Köcher fest, dass keine emotionale Abkehr von der Tätigkeit stattfindet: „Die Erinnerung an die früher genossenen Gratifikationen des Lesens bleiben bei vielen Abbrechern außerordentlich lebendig und lassen die Mehrheit wünschen, zu diesem Interesse zurückzukehren. “71 Im Gedächtnis repräsentierte Belohnungen sind v. a. Entspannung und Ablenkung. ${ }^{72}$

Allgemein haben Lebensereignisse wie berufliche Veränderungen oder Familiengründung einen Einfluss darauf, ob eine Tätigkeit ausgeübt wird. ${ }^{73}$ Der Tätigkeitsverzicht ist dann eine Reaktion auf situative Umstände (zu wenig Zeit, zu erschöpft) abgesehen von der (bewussten) Anpassung des Verhaltens an neue Ziele. ${ }^{74}$ Die Leseintensität wird also von der Lebenssituation beeinflusst, sodass sich Phasen intensiver und geringer/ausbleibender Lesetätigkeit und damit Prozesse der Distanzierung und Annäherung an den Gegenstand abwechseln. ${ }^{75}$ Knapp die Hälfte der Abbrecher hatte wiederholt Phasen, in denen kaum gelesen und dann doch wieder gelesen wurde. ${ }^{76}$ Ebenso wie Lebensumstände die Ausübung beeinträchtigen, können sie zugleich die Rückkehr zum Lesen begünstigen. Zum Beispiel durch Urlaub, Fertigstellung von Ausbildung/Studium, weniger Unternehmungen mit Freunden, keiner ausschließlichen Fokussierung mehr auf den Beruf oder den Wechsel in den Ruhestand. ${ }^{77}$ Am häufigsten stimmen Rückkehrer jedoch der Aussage zu, dass Lesebedürfnisse unabhängig von situativen Umständen durch ein bestimmtes Buch gegenständlich wurden und sie deshalb wieder angefangen haben zu lesen. ${ }^{78}$

\footnotetext{
${ }^{68}$ Vgl. Köcher (1993), S. 280.

${ }^{69}$ Vgl. Köcher (1993), S. 268.

${ }^{70}$ Vgl. Köcher (1993), S. 269; Hierbei spielen auch Persönlichkeitsmerkmale eine Rolle (vgl. Köcher (1993), S. 218).

${ }^{71}$ Köcher (1993), S. 218

${ }^{72}$ Vgl. Köcher (1993), S. 281.

${ }^{73}$ Vgl. Köcher (1993), S. $241 \mathrm{f}$.

${ }^{74}$ Vgl. Naab (2013), S. 81.

${ }^{75}$ Vgl. Köcher (1993), S. 236; vgl. auch Häuser (1995), S. 50 f.

76 Vgl. Köcher (1993), S. 236.

77 Vgl. Naab (2013), S. 79; vgl. Köcher (1993), S. 294 ff.

78 Vgl. Köcher (1993), S. 294
} 
Gemäß dem Value-in-Use-Verständnis wird ein Urteil über ein Angebot immer relativ getroffen. Demnach bewerten Kunden ihre Erlebnisse vor dem Hintergrund ihrer Erfahrungen mit anderen Angeboten. Außerdem reflektieren Kunden ihr eigenes Leseverhalten und entscheiden, ob sie im Alltag Situationen zum Lesen wahrnehmen oder nicht. Im empirischen Teil der Arbeit wird geprüft, ob sich Vergleichsprozesse in den Kundenaussagen nachweisen lassen. 


\section{Bereitstellungsqualität von Büchern}

\subsection{Definition der Bereitstellungsqualität}

Die jeweilige Erscheinungsform von Angeboten in der Realität wird durch die Kombination der Handlungsparameter eines Anbieters bestimmt. Die konkrete Gestaltung eines Angebots ist einzigartig und wird als Betreibungskonzept bezeichnen. ${ }^{79}$ Heinonen et al. verstehen unter einem Angebot ,a generic concept for the holistic entity the provider has designed and intends to provide/sell, containing material and immaterial elements to achieve its own goals“ ${ }^{80}$ Es werden nur solche Parameter betrachtet, die das Erscheinungsbild aus Kundensicht prägen und auf die der Anbieter Einfluss nehmen kann. Zu diesen gehört z. B. das absatzpolitische Instrumentarium (Marketingmix) ${ }^{81}$ bzw. insbesondere die handelsbetriebliche Leistungspolitik. ${ }^{82}$ Die Freiheit bei der Angebotsgestaltung soll in dieser Arbeit jedoch nicht anhand der absatzpolitischen Instrumente untersucht werden, sondern zur Analyse der Angebote wird auf das Konzept der Bereitstellungsqualität von Medien als Bezugsrahmen zurückgegriffen. Der Grund dafür ist, dass nicht die Anbieterziele und seine gewählten Mittel zur Zielerreichung untersucht werden sollen, sondern die Ziele der Kunden und wie der Anbieter diese bei der Angebotsgestaltung berücksichtigen kann. Ähnlich verfährt Purper bei der Entwicklung von Betriebsformen im Handel aus Kundensicht. Er bestätigt, dass sich Angebote unterschiedlich gut zur Befriedigung folgender Kundenmotive eignen: sich stimulieren lassen und länger verweilen (Erlebnis), niedrige Preise, hoher Servicegrad sowie Auswahl aus einer großen Anzahl an Optionen. ${ }^{83}$

Der Begriff „Bereitstellungsqualität“ wurde von Saxer eingeführt, um u. a. das Leistungsvermögen von Medien aus gesellschaftlicher Sicht miteinander vergleichen zu können. ${ }^{84}$ Das Konzept ist somit von seiner Entstehungsgeschichte her als Vergleichsmaßstab angelegt, der theoretisch auf alle Angebotsformen angewendet werden kann (vgl. Kap. 2). Saxers Überlegungen basieren auf Platte, der Bereitstellung nicht nur bei den Produzenten verortet, sondern auch bei Einrichtungen, die den Zugang zu den Medien herstellen (z. B. Buchhandlungen). ${ }^{85}$ Nach Platte endet der Bereitstellungsvorgang mit dem öffentlichen Angebot der Medien. ${ }^{86}$ Die

\footnotetext{
${ }^{79}$ Vgl. Ahlert/ Olbrich (1999), S. 1.

${ }^{80}$ Heinonen/ Strandvik (2015), S. 478.

${ }^{81}$ Vgl. Müller-Hagedorn (1995), S. 239 f.

${ }^{82}$ Vgl. Barth/ Hartmann/ Schröder (2015), S. 48.

${ }^{83}$ Vgl. Purper (2007), S. 195.

${ }^{84}$ Vgl. Saxer (1972), S. 124 f.

${ }^{85}$ Vgl. Platte (1965), S. 28, S. 30 f.

${ }^{86}$ Vgl. Platte (1965), S. 17.
} 
Arbeit konzentriert sich auf den Endpunkt der Bereitstellung und setzt die kreative Schöpfung sowie Buchherstellung und -veröffentlichung voraus. Somit ist Bereitstellungsqualität im weiten Sinne angesprochen, d.h. die Analyse von Qualitätsmerkmalen eines Angebots, die Kunden zur Beurteilung heranziehen. In Abgrenzung dazu steht die Bereitstellungsqualität im engen Sinne. Hier stehen Eigenschaften und technische Bedingungen des Buches im Mittelpunkt. Sie entspricht dem Forschungsverständnis der Buchwissenschaft, gemäß dem Bereitstellungsqualität „die Eigenschaften der Materialobjekte der Schriftkommunikation, ihrer Träger und Übermittlungsmedien (Beschreibstoffe, Bedruckstoffe, Elektronisches Papier mit varianter Anzeigetechnologie), Typographie, Buchmalerei, Buchillustration, Bucheinband, Bildschirmtypographie, E-Book ${ }^{\star 87}$ umfasst. In einer früheren Arbeit erklärt Saxer, dass er mit Bereitstellungsqualität meint „wie leicht und in welcher Vielfalt sie (Medien; Anm.) räumlich und zeitlich zugänglich sind. “88 In Bezug auf die konkreten Dimensionen der Bereitstellungsqualität erwähnt Saxer Zeichensystem(e), Inhalte, Periodik der Erscheinungsweise, technische Formate, Bereitstellungsdauer, Bereitstellungsräume und Bereitstellungskosten ${ }^{89}$ sowie den Bedienungsaufwand. ${ }^{90}$ Vergleicht man diese mit denjenigen aus der Definition, ergibt sich ein Interpretationsspielraum. Aus der Definition lassen sich konkret die Dimensionen inhaltliche Vielfalt, Leichtigkeit des Zugangs, räumlicher Zugang und zeitlicher Zugang ableiten. An dieser Stelle ist der Hinweis wichtig, dass es Saxer und Platte u. a. darum geht, Unterschiede zwischen Medien herauszuarbeiten. ${ }^{91}$ Da die Arbeit jedoch eine Kundenperspektive einnimmt, werden die Dimensionen Zeichensystem, Periodizität und Inhalt als Einheit betrachtet. Die Dimension wird als „Inhaltliche Vielfalt“ bezeichnet und um den Aspekt der Ordnung der Bücher ergänzt. Für die Leichtigkeit des Zugangs und den Bedienungsaufwand wird die Dimension „Leichtigkeit der Benutzung“ geschaffen. Das Merkmal „technisches Format“ wird mit der Dimension „Basistechnologie“ und der Ausprägung Druck/ elektronische Aufbereitung abgedeckt. ${ }^{92}$ Die Abb. 4.1 zeigt die Dimensionen im Überblick.

\footnotetext{
${ }^{87}$ Rautenberg (2015).

88 Saxer (1975), S. 222.

${ }^{89}$ Vgl. Saxer (2010), S. 89.

90 Vgl. Saxer (2012), S. 141.

${ }^{91}$ Vgl. Platte (1965), S. 16; vgl. auch Dahinden/ Trappel, (2010) S. 440.

${ }^{92}$ Vgl. Dahinden/ Trappel (2010), S. 439 f.
} 


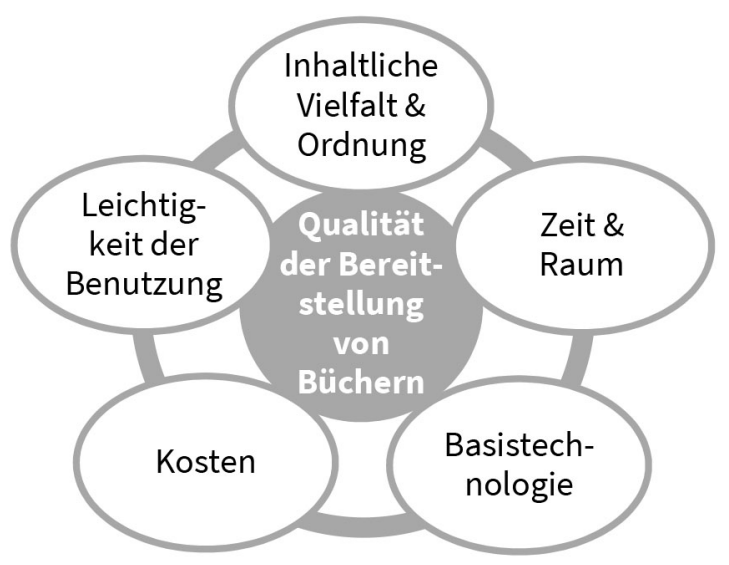

Abb. 4.1 Dimensionen der Bereitstellungsqualität von Büchern (Quelle: Eigene Erstellung)

Einer Dimension lassen sich Qualitätsmerkmale zuordnen, die nachfolgend diskutiert werden.

Im empirischen Teil der Arbeit wird untersucht, ob die theoretisch identifizierten Qualitätsmerkmale einen Realitätsbezug haben, indem geprüft wird, ob Kunden sich bei ihrer Urteilsbildung auf diese stützen. Durch die Festlegung von beobachtbaren Merkmalen können diese bei der Inhaltsanalyse bestimmten Kundenaussagen (Indikatoren) zugeordnet werden. ${ }^{93}$

Zusammenfassend lässt sich Bereitstellungsqualität aus Kundensicht folgendermaßen definieren:

Die Bereitstellungsqualität ist das Ergebnis eines Kundenurteils, in das einfließt, unter welchen technologischen Bedingungen, in welchem zeitlichen und räumlichen Umfang, in welcher inhaltlichen Vielfalt und Ordnung und zu welchen Kosten Bücher zugänglich sind und wie leicht das Angebot zu benutzen ist.

\subsection{Bereitstellungsdimension: Inhaltliche Vielfalt und Ordnung der Bücher}

Als ein Ordnungsprinzip kann die Sortimentsbildung aufgefasst werden, d. h. der Anbieter trifft eine Vorauswahl aus allen Titeln auf dem Markt mit Blick auf die vermuteten Präferenzen der eigenen Kunden (vgl. Abb. 4.2).

\footnotetext{
${ }^{93}$ Vgl. Wagner et al. (2008), S. 58.
} 

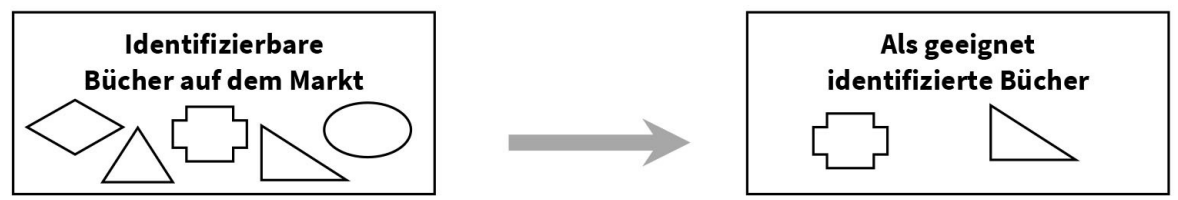

Abb. 4.2 Visualisierung von „Selektion“ (Quelle: Eigene Erstellung nach Walter (2007), S. 83)

Bei Online-Katalogen besteht keine Raumknappheit, sodass die Sortimentsbildung unterbleiben kann, was eine große Anzahl an Optionen zur Folge hat, zwischen denen Kunden wählen können bzw. müssen. ${ }^{94}$ Je mehr Links sichtbar sind bzw. je mehr Bücher im Raum präsentiert werden, desto komplexer ist die Umgebung. ${ }^{95} \mathrm{Im}$ Allgemeinen steigt der Aufwand mit der wahrgenommenen Komplexität. ${ }^{96}$ Die Komplexität kann zu kognitivem und/oder emotionalem Overload führen, was die Schwierigkeit, eine Entscheidung zu treffen, erhöht. ${ }^{97}$ Bei einem großen Sortiment ist die Wahrscheinlichkeit von Overload höher als bei weniger Optionen. ${ }^{98}$ Ein Händler kann dem Kunden also helfen, den psychischen Suchaufwand zu reduzieren. ${ }^{99}$ Hieran anknüpfend erklärt Riethmüller (Geschäftsführer Osiander), dass eine Buchhandlung dem Kunden das Auswählen von Büchern erleichtert, weil dieser durch die Selektion Zeit und Mühe spart. ${ }^{100}$ Folglich eignet sich der Facheinzelhandel aus Kundensicht mittel bis schlecht, wenn eine große Auswahl gewünscht wird. ${ }^{101}$ Dazu passt das Ergebnis, dass für Kunden in Buchhandlungen eine große Auswahl im Vergleich zu allen abgefragten Merkmalen nur von mittlerer Wichtigkeit ist. $^{102}$

\footnotetext{
${ }^{94}$ Vgl. Hagenhoff (2015), S. 529; vgl. Heeter (1989), S. 226.

${ }_{95}$ Vgl. Schweiger (2007), S. 56; vgl. Müller/ Schrepp (2013), S. 214.

${ }^{96}$ Vgl. Wirth/ Schweiger (1999), S. 60.

${ }^{97}$ Vgl. Diehl/ Poynor (2010), S. 18.

${ }^{98}$ Vgl. Diehl/ Poynor (2010), S. 29.

${ }^{99}$ Vgl. Müller-Hagedorn/ Toporowski/ Zielke (2012), S. $712 \mathrm{f}$.

${ }^{100}$ Vgl. Graf (2014), ab 10. Min., https://www.youtube.com/watch?v=j9u7FD1elAA.

${ }^{101}$ Vgl. Purper (2007), S. 202, S. 207.

${ }^{102}$ Vgl. Börsenverein (Hrsg.) (2015), S. 182; vgl. Schwiering (2011), S. 8; vgl. IfH (2010), S. 29 f.
} 

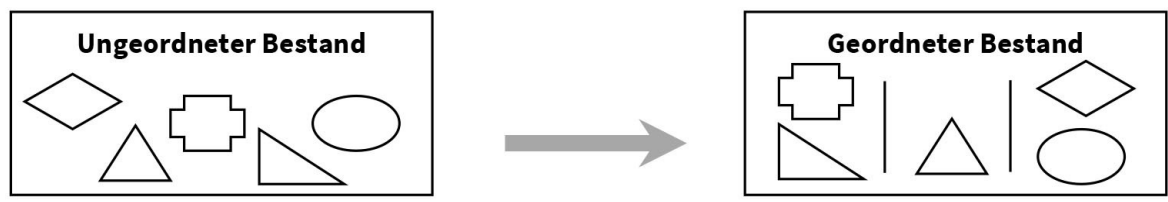

Abb. 4.3 Visualisierung von „Zusammenfassung“ (Quelle: Eigene Erstellung nach Walter (2007), S. 83$)^{103}$

Die Komplexität kann durch Strukturierung kompensiert werden (vgl. Abb. 4.3). ${ }^{104}$ Hierbei ist aus Kundensicht wichtig, welche Ordnungsprinzipien der Anbieter zur Gruppenbildung nutzt, da sich dies u. a. auf die Zusammenstellung von Büchern im Raum auswirkt ${ }^{105}$ sowie auf die Anzahl an Kategorien in Online-Katalogen. Auch hilft es Kunden, wenn der Anbieter berücksichtigt, welche Ordnungskriterien diesem bekannt sind, ${ }^{106}$ denn ob die Struktur als logisch empfunden wird, hängt von den Erwartungen der Kunden ab, d. h. von den Kriterien, die diese üblicherweise zur Ordnung heranziehen. ${ }^{107}$

Merkmal einer Dimension von Bereitstellungsqualität: Strukturierung des Buchbestands

Sommer/Rimscha schlagen vor, den Inhalt gemäß den Erwartungen der Nutzer zu typisieren. ${ }^{108}$ Zum einen können Kunden Inhalte nach dem erwarteten Realitätsbezug voneinander abgrenzen. Demnach lassen sich fiktionale (fantasiebetont; reale Gegebenheiten in einer imaginierten Welt) und faktuale (tatsachenbetont; Gegebenheiten in der Realität) Inhalte unterscheiden. ${ }^{109}$ Fiktionale Inhalte können zusätzlich in Genre unterteilt werden. Beispiele für Genreliteratur bei Romanen sind Abenteuer, Liebe, Krimi, Thriller, Horror/Grusel, Fantasy und Science Fiction. ${ }^{110}$ Durch die Genrezuordnung können Kunden sich einen Eindruck vom Inhalt verschaffen, ohne ihn zu kennen. ${ }^{111}$ Mit der Zuschreibung eines Genres ist aber die Erwartung des Kunden verbunden, dass der Inhalt typische Merkmale des Genres oder ein typisches Leseerleben aufweist. ${ }^{112}$ Ferner lassen sich Inhalte nach ihrer

\footnotetext{
${ }^{103}$ Walter verwendet das Wort Aggregation, das jedoch eher die Bedeutung von addieren oder verdichten hat.

${ }^{104}$ Vgl. Wirth/ Schweiger (1999), S. 60.

${ }^{105}$ Vgl. Müller-Hagedorn/ Toporowski/ Zielke (2012), S. 703; vgl. Haller/ Fabian (2004), S. 222.

${ }^{106}$ Vgl. Nielsen/ Badiu (2013), S. 153; vgl. Wirth/ Schweiger (1999), S. 56).

107 Vgl. Kalyanaraman/ Wojdynski (2015), S. 434; vgl. Nielsen/ Badiu (2013), S. 148 f.

108 Vgl. Sommer/ Rimscha (2014), S. 254

109 Vgl. Dahinden/ Trappel (2010), S. 463; vgl. Jesch (2009), S. 73 f.; vgl. Sommer/ Rimscha (2014), S. 254; vgl. Lünenborg (2013), S. 88.

${ }^{110}$ Vgl. Pohl/ Umlauf (2007), S. 65 ff.

111 Vgl. Gehrau (2003), S. 219.

${ }^{112}$ Vgl. Gehrau (2003), S. 214, S. 217.
} 
Leserreichweite unterteilen, d. h. von welchen Lesertypen Bucharten gelesen werden (vgl. Abb. 4.4). Bei dieser aus empirischen Ergebnissen hergeleiteten Unterteilung fällt auf, dass insbesondere Bücher mit starkem Bezug zu Alltagsthemen von vielen Lesern, d. h. auch von Wenig- und Durchschnittslesern genutzt werden.

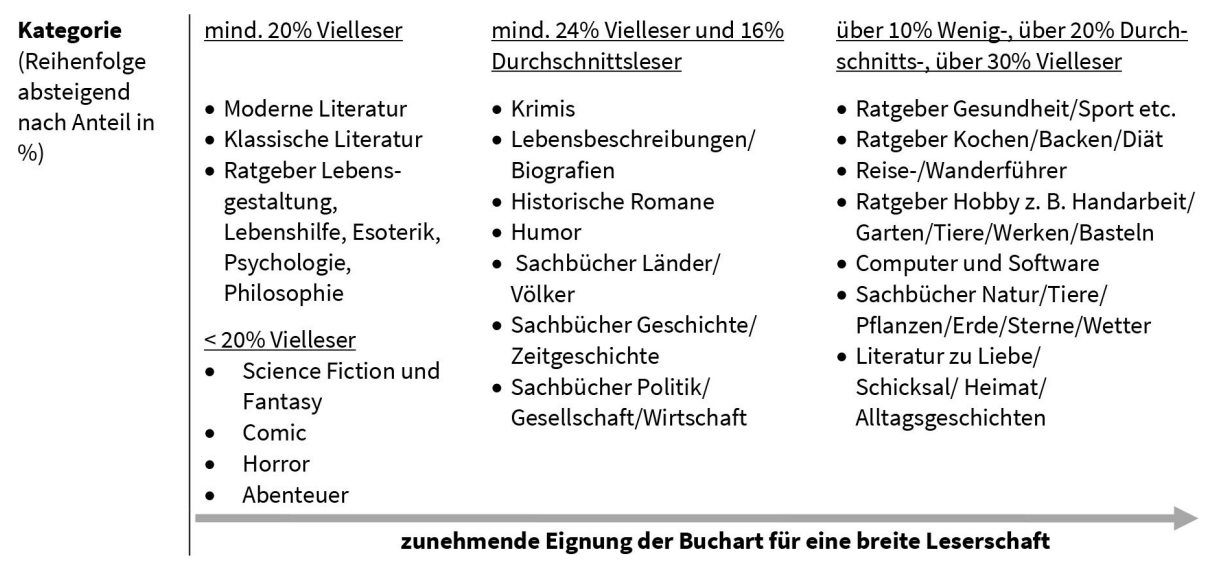

Abb. 4.4 Bucharten nach Leserreichweite, mind. gelegentliche Nutzung (Quelle: Eigene Erstellung nach Ergebnissen von Stiftung Lesen (2008), S. 168 - 175)

Unter den E-Book-Lesern sind belletristische Bücher am stärksten verbreitet (79 \%). Ratgeber lesen hin und wieder knapp $40 \%$, knapp $37 \%$ Lehrbücher/wissenschaftliche Literatur, knapp $27 \%$ Reiseführer, $12 \%$ Comics und knapp $10 \%$ sonstige Sachbücher. ${ }^{113}$ Im Gegensatz zum Gesamtbuchmarkt zeigt sich ein Schwerpunkt bei fiktionalen Inhalten. Insgesamt wird angenommen, dass Kunden bewerten, aus welcher Vielfalt an Bucharten sie auswählen können. Auch für Kunden in Buchhandlungen ist die Vielfalt an Bucharten wichtig. ${ }^{114}$

Merkmal einer Dimension von Bereitstellungsqualität: Vielfalt der Bucharten im Bestand

Zum anderen orientieren sich Leser an der Periodik der Erscheinungsweise. Sowohl faktuale als auch fiktionale Inhalte können Seriencharakter aufweisen, d. h. fortlaufend produziert werden. ${ }^{115}$ Die regelmäßige Rezeption bei Serien fördert die Bindung an diese. ${ }^{116}$ Durch die Periodik der Erscheinungsweise wird ein ,typischer" Ausübungsrhythmus der Lesetätigkeit nahegelegt. ${ }^{117}$ Nutz et al. bestätigen, dass bei Serien der Anteil an Viellesern teilweise sehr hoch ist. Ihre Studie zeigt zudem, dass

\footnotetext{
113 Vgl. Bitkom (2015), S. 13.

114 Vgl. Schwiering (2011), S. 8.

115 Vgl. Sommer/ Rimscha (2014), S. 257.

116 Vgl. Wirth/ Schramm (2010), S. 582.

117 Vgl. Bonfadelli/ Fritz (1993), S. 35.
} 
sich die Werte je nach Genre unterscheiden. Bei wöchentlich erscheinenden Romanserien zählen beim Genre Science Fiction/Zukunft $71 \%$ zu den Viellesern, beim Krimi $43 \%$, bei Liebe $68 \%$, bei Western $42 \%$ und bei Grusel $52 \% .{ }^{118}$ Hieraus lässt sich folgern, dass Serienleser vermutlich die Verfügbarkeit ihrer Serie erwarten.

Merkmal einer Dimension von Bereitstellungsqualität: Verfügbarkeit von Serien und Reihen

Gemäß Purper kann ein umfangreicher Bestand ein Qualitätsmerkmal sein (vgl. Kap. 4.1). Die Folgen einer großen Auswahl sind jedoch nicht immer vorteilhaft für Kunden. Nach dem Expectation-Disconfirmation-Mechanismus ist Zufriedenheit an die (Nicht-)Bestätigung von Erwartungen geknüpft. ${ }^{119}$ Personen bilden Erwartungen im Hinblick auf die Bestandsgröße, d. h. sie treffen eine Vorannahme, wie sicher sie eine Option in einer gegebenen Menge an Optionen finden werden, die mit ihren Präferenzen übereinstimmt. ${ }^{120}$ Die Erwartungs- bzw. Anspruchshaltung, eine ideale Option zu finden, fällt bei großen Sortimenten höher aus als bei kleineren. ${ }^{121}$ Kunden glauben also, bei einem großen Sortiment eine bessere Option finden zu können.

\section{Merkmal einer Dimension von Bereitstellungsqualität: Größe des Bestands}

Allerdings entspricht die ausgewählte Option aus einem großen Sortiment im Vergleich signifikant schlechter den eigenen Erwartungen. Die höheren Erwartungen erfüllen sich also nicht, was sich negativ auf die Zufriedenheit mit der gewählten Option auswirkt. ${ }^{122}$ Insgesamt beeinflusst also die Zufriedenheit (Passung mit den eigenen Präferenzen), ob Kunden den Bestand als groß genug erleben, sodass auch eine geringere Anzahl an Optionen ausreichend sein kann.

Merkmal einer Dimension von Bereitstellungsqualität: Übereinstimmung mit Präferenzen

Für Kunden in Buchhandlungen ist zudem die Verfügbarkeit der gesuchten Bücher wichtig. ${ }^{123}$ Die Erwartung, das Gesuchte zu finden, ist aus Anbietersicht ein Enttäuschungsrisiko, das unvermeidbar ist. Knapp die Hälfte der Bevölkerung stimmt der Aussage zu, dass sie in einer Buchhandlung nichts gekauft hat, weil das gesuchte Buch nicht vorrätig/nicht auf Lager war. ${ }^{124}$ Zudem können Misserfolge (Fehlentscheidungen) das lesebezogene Selbstkonzept abwerten. ${ }^{125}$ Die Wichtigkeit positiver Leseerlebnisse erhöht vermutlich das Anspruchsniveau bei der Buchauswahl. Vom

\footnotetext{
118 Vgl. Nutz/ Schlögell (1991), S. 161.

119 Vgl. Diehl/ Poynor (2010), S. 8.

120 Vgl. ebd.

121 Vgl. Diehl/ Poynor (2010), S. 13, S. 22, S. 30.

122 Vgl. Diehl/ Poynor (2010), S. 13, S. 22, S. 30.

123 Vgl. Börsenverein (Hrsg.) (2015), S. 182; vgl. Schwiering (2011), S. 8; vgl. IfH (2010), S. 29 f.

124 Vgl. Börsenverein (Hrsg.) (2015), S. 189.

${ }^{125}$ Vgl. Schiefele/ Möller (2004), S. $116 \mathrm{f}$.
} 
Anspruchsniveau hängt es wiederum ab, „welche Alternativen als zufrieden stellend angesehen und akzeptiert werden und welche zurückgewiesen werden. “126 Dass Kunden nichts Passendes finden, ist die zweithäufigste Ursache für den Nichtkauf in einer Buchhandlung. ${ }^{127}$ Eine fehlende Passung des Bestands mit Präferenzen lässt sich demnach auf die fehlende Verfügbarkeit von gesuchten Büchern, aber auch auf ein hohes Anspruchsniveau zurückführen. Weitere Qualitätsmerkmale können sein: die Verfügbarkeit von Novitäten bzw. die Schnelligkeit, mit der aktuelle Bücher im Bestand zugänglich sind, die Verfügbarkeit von populären Büchern („Bestseller“) sowie die Verfügbarkeit von fremdsprachigen Buchtiteln, was sich jeweils aus den Erwartungen von Buchhandelskunden in Buchhandlungen bzw. beim Merkmal Neuerscheinungen aus den Erwartungen von Bibliothekskunden in Bibliotheken schlussfolgern lässt. ${ }^{128}$

Merkmale einer Dimension von Bereitstellungsqualität: Aktualität der Bücher im Bestand, Bekanntheit/Popularität der Bücher im Bestand, Fremdsprachige Bücher im Bestand

\subsection{Bereitstellungsdimension: Räumlicher und zeitlicher Zugang}

\subsubsection{Allgemeine Bereitstellungsmodi von Büchern}

Hier sind die Wege angesprochen, wie ein Kunde mit den Büchern in Kontakt kommen kann. Im Handel werden vier Möglichkeiten unterschieden, wie ein Anbieter den Kontakt zu seinen Kunden gestalten kann: Residenzprinzip (Kunde kommt zum Händler), Domizilprinzip (Händler kommt zum Kunden), Treffprinzip (Händler und Kunde treffen sich an einem Ort) und Distanzprinzip (Händler und Kunde stehen nicht im physischen Kontakt). ${ }^{129}$ Eine ähnliche Einteilung schlägt Saxer vor. Er benennt drei Bereitstellungsmodi, die sich danach unterscheiden, auf welche Weise der Medienkontakt zustande kommt: Besuchsprinzip, Bring-Prinzip und Abrufprinzip (vgl. Abb. 4.5). ${ }^{130}$ In allen Fällen geht die Initiative vom Kunden aus, jedoch bieten Bereitstellungsmodi unterschiedlich viel Flexibilität. Beim Bringund Abrufprinzip erfolgt die Bereitstellung beim Kunden, d. h. die Bücher bewegen sich zu ihm, während beim Besuchsprinzip der Kunde den Bereitstellungsort aufsucht, sich also dorthin bewegt.

\footnotetext{
${ }^{126}$ Vgl. Kroeber-Riel/ Gröppel-Klein (2013), S. 481.

127 Vgl. Börsenverein (Hrsg.) (2015), S. 182.

128 Vgl. Schwiering (2011), S. 8; vgl. Institut für Demoskopie Allensbach (Hrsg.) (2016), S. 13.

129 Vgl. Barth/ Hartmann/ Schröder (2015), S. 88 f.

130 Saxer (1972), S. 132; Saxer unterscheidet Abruf-, Zulieferungs- und Abholprinzip. Das Abholprinzip ist in seinem Bedeutungsgehalt jedoch etwas einengend, da z. B. ein Buch auch vor Ort in einer Bibliothek gelesen werden kann.
} 

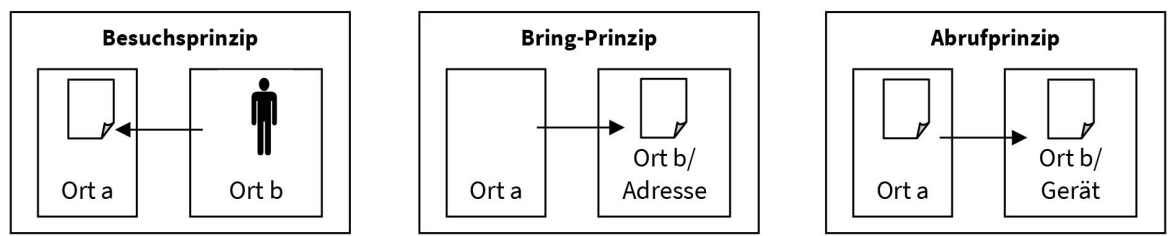

Abb. 4.5 Visualisierung der Bereitstellungsmodi (Quelle: Eigene Erstellung inspiriert von Walter (2007), S. 92)

Die Bereitstellung im Sortimentsbuchhandel und in Bibliotheken ist durch das Besuchsprinzip gekennzeichnet. ${ }^{131}$ Hier trifft der Anbieter die Entscheidung über den Ort der Bereitstellung (Standort) und zu welcher Zeit die Bücher zugänglich sind (Öffnungszeiten). ${ }^{132}$ Für die Kunden reduziert sich der wahrgenommene Aufwand, wenn sie den Besuch mit anderen Aktivitäten kombinieren können. Beispielsweise geben Bibliothekskunden an, den Aufenthalt häufig mit dem Einkauf von Lebensmitteln zu verbinden. ${ }^{133}$ Durch Automaten können für bestimmte Funktionen zeitliche Einschränkungen aufgehoben werden wie Abholung und Rückgabe von Büchern, wenn Öffnungszeiten nicht erweitert werden sollen.

Beim Bring-Prinzip muss der Anbieter die Bereitstellung mittels Transport organisieren, während er beim Abrufprinzip bestimmte Übertragungstechniken nutzt. ${ }^{134}$ Bring- und Abrufprinzip unterscheiden sich nach der Art der Speicherung der Bücher. Die Speicherung auf materiellen Objekten wie Papier erfordert das Hinbringen der Objekte, also den Transport, während Bücher bei einer elektronischen Aufbereitung auf einem Rechner gespeichert sind, der in einem Netz wie dem Internet fest installiert ist. ${ }^{135}$ Alle Geräte, die an das Internet angeschlossen sind, können zu jeder Zeit und von jedem Ort aus auf die Buchdaten zugreifen, weil die physische durch virtuelle Bewegung ersetzt wird. ${ }^{136}$ Nach Platte ist die räumliche Bereitstellungsqualität am höchsten, wenn die Bereitstellung der Medien im Lebensbzw. Privatraum des Kunden erfolgt. ${ }^{137}$ Bei virtueller Mobilität kann der Bereitstellungsraum quasi an allen Orten Gestalt annehmen. Diese Situation wird mit Ubiquität (Überallverfügbarkeit der Bücher) umschrieben. ${ }^{138}$ Auch ist die Geschwindigkeit der Bereitstellung beim Abrufprinzip am höchsten. ${ }^{139}$ Das Abruf-

\footnotetext{
${ }^{131}$ Vgl. Barth/ Hartmann/ Schröder (2015), S. 91.

132 Vgl. Platte (1965), S. 35.

133 Vgl. Messerschmidt/ Naskowski (2011), S. 68 - 71, S. $102-105$.

${ }^{134}$ Vgl. Platte (1965), S. 26, S. 34; vgl. Dahinden/ Trappel (2010), S. 439; vgl. Mowshowitz (1992), S. 237.

135 Vgl. Freyer (2013), S. 57 f.

${ }^{136}$ Vgl. Freyer (2013), S. 57; vgl. Schmoll/ Tiemann/ Welzel (2014), S. 6 f.

137 Vgl. Platte (1965), S. 29, S. 33 f., S. 139.

138 Vgl. Kuhn/ Hagenhoff (2015), S. 322.

139 Vgl. Mowshowitz (1992), S. 237.
} 
prinzip bedeutet somit eine erhöhte Flexibilität für den Kunden im Vergleich zum Bring-Prinzip und Besuchsprinzip. ${ }^{140}$

Merkmal einer Dimension der Bereitstellungsqualität: Freiheit beim Lesebeginn

Das Abrufprinzip profitiert von einer flächendeckenden Versorgung der Bevölkerung mit schnellen und mobilen Internetzugängen. Damit sind Anbieter auf die bereitgestellte Infrastruktur der Netzbetreiber und auf Leistungen von Telekommunikationsunternehmen angewiesen. ${ }^{141}$ Beim Abrufprinzip lassen sich noch die Bereitstellungsverfahren der Online-Angebote unterscheiden.

\subsubsection{Bereitstellungsverfahren von Büchern bei Online-Angeboten}

Bei Online-Angeboten handelt es sich um verteilte Anwendungen mit einer Software-Architektur, die das Client-Server-Prinzip nutzt. ${ }^{142}$ Das Client-Server-Prinzip ist das allgemeinste Konzept der verteilten Verarbeitung von Daten, die über Netze ausgetauscht werden. ${ }^{143}$ Ein Rechner, das als Server agiert, hält Daten vor und stellt diese auf Anfrage über das Netz bereit, während das Client-Programm auf dem Gerät des Kunden die Daten anfordert und erhält. ${ }^{144}$

Die Aufgaben der Programmausführung (Rechenlast) und Datenspeicherung können zwischen Server und Kundengerät (Client) unterschiedlich verteilt werden. ${ }^{145}$ Die Bestandteile der Architektur lassen sich in drei Schichten unterteilen. Die Datenhaltungsschicht enthält die Daten, auf denen die Software ihre Funktionalität anwendet, also u. a. die Buchdaten (Speicherort). Die Funktionsschicht enthält die Funktionalität der Software, also die Verarbeitungslogik. Die Präsentationsschicht stellt die Schnittstelle zum Kunden dar, also die Benutzungsoberfläche einer Software. ${ }^{146}$ Im Folgenden werden die Bereitstellungsverfahren erläutert (vgl. Abb. 4.6).

\footnotetext{
${ }^{140}$ Vgl. Schmoll/ Tiemann/ Welzel (2014), S. 7.

${ }^{141}$ Vgl. Braun (2014), S. 62 ff.

142 Vgl. Dahinden/ Trappel (2010), S. 440.

143 Vgl. Leimeister (2015), S. 107.

144 Vgl. Freyer (2013), S. 168.

${ }^{145}$ Vgl. Tornack et al. (2011), S. 19; vgl. Leinmeister (2015), S. 106; vgl. Hagenhoff (2014), S. 13.

146 Vgl. Hagenhoff (2014), S. 12.
} 


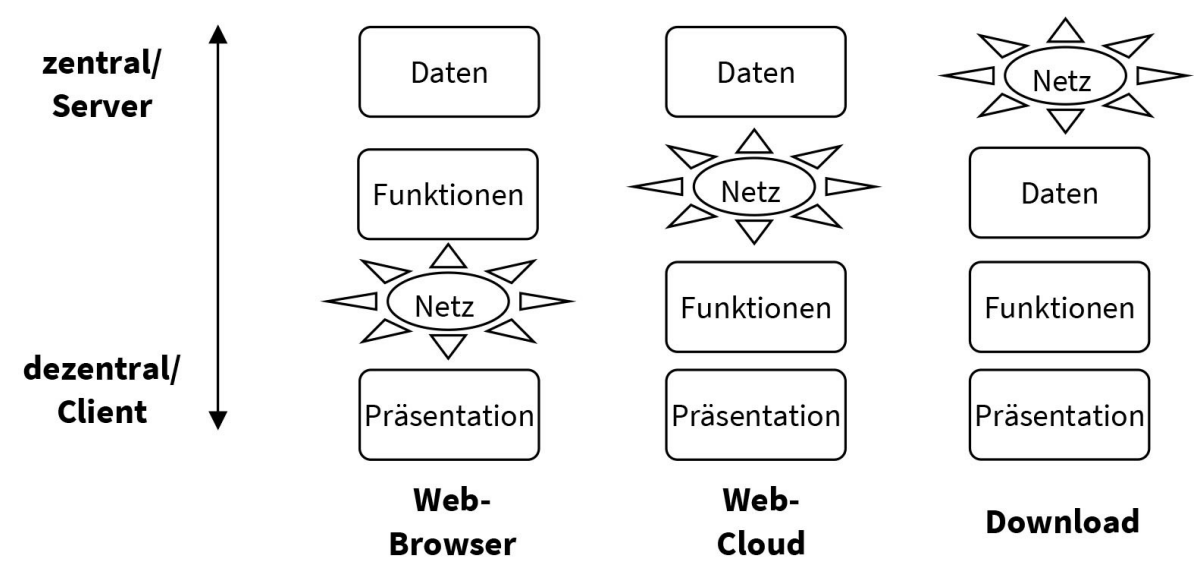

Abb. 4.6 Wesentliche Formen der Arbeitsaufteilung zwischen Server und Client (Quelle: Eigene Erstellung in Anlehnung an Leimeister (2015), S. 108 und Hagenhoff (2014), S. 15)

Bei der Bereitstellung über einen Web-Browser befindet sich nur die Präsentationsschicht auf dem Gerät des Kunden, während die Funktions- und Datenschicht auf dem Server des Anbieters verbleiben. Dieses Verfahren wird allgemein bei Webseiten und z. B. beim Angebot Google Books des Unternehmens Google angewendet. Der Kunde muss für die Bereitstellung und für das Lesen der Bücher keine spezielle Anbieter-Software installieren. ${ }^{147}$ Anders ist dies bei der Web-Cloud. Bei diesem Verfahren liegen sowohl die Präsentations- als auch die Funktionsschicht auf dem Gerät des Kunden, während die Datenschicht auf dem Server des Anbieters verbleibt. ${ }^{148}$ Dies führt dazu, dass der Kunde im Vorfeld eine Software des Anbieters auf seinem Gerät installieren muss, um auf die Daten zugreifen können. Wenn Kunden Bücher mittels Browser oder Web-Cloud lesen, wird von Online-Lesen gesprochen, weil eine dauerhafte Netzverbindung nötig ist. Der Begriff Cloud wird zur Abgrenzung von einer Buchsammlung verwendet, die auf einem Gerät gespeichert ist. Beim Download liegen alle Schichten auf dem Gerät des Kunden, aber die Datenschicht ist von der Präsentations- und Funktionsschicht getrennt. ${ }^{149}$ Eine Datei wird vollständig auf ein Gerät übertragen und dort gespeichert, sodass der Kunde offline auf das Buch zugreifen kann. ${ }^{150}$ In diesem Fall ist von Offline-Lesen die Rede. Der Zugriff auf das Buch ist je nach Dateiformat entweder nur mit Anbieter-Software oder mit beliebiger Software möglich. Es wird vermutet, dass Kunden die Möglichkeit des OfflineLesens wichtig ist, da im Alltag nicht immer eine Internetverbindung - insbesondere beim Unterwegs-Lesen - gewährleistet ist.

\footnotetext{
147 Vgl. Kuhn/ Hagenhoff (2015), S. 322.

148 Vgl. Hagenhoff (2014), S. 14.

149 Vgl. ebd.

${ }^{150}$ Vgl. Freyer (2013), S. 14, S. 57, S. 150 f.; vgl. Kuhn/ Hagenhoff (2015), S. 322.
} 


\subsection{Bereitstellungsdimension: Technologische Bedingungen des Zugangs}

\subsubsection{Geräte zum Bücherlesen}

Mit Drucktechnologie geht einher, dass Kunden kein Gerät zum Lesen benötigen. ${ }^{151}$ Neben der Wichtigkeit der äußeren Merkmale von gedruckten Bücher ${ }^{152}$, welche die operationale (sinnliche) Ebene einer Tätigkeit betreffen ${ }^{153}$, ist ein wichtiger Grund für das Nichtlesen von E-Books die Abhängigkeit von der Akkulaufzeit eines Geräts. ${ }^{154}$ Janneck et al. fanden in ihrer qualitativen Studie heraus: Selbst wenn Untersuchte einen E-Reader mit langer Betriebszeit nutzen und es nicht zu Leseunterbrechungen durch einen entleerten Akku kommt, „stellt die Abhängigkeit vom Akku offenbar eine Art latente Bedrohung dar, die das Lesevergnügen beeinträchtigt. “ 155

Betrachtet man die Absatzentwicklung der Gerätetypen, die sich zum Buchlesen eignen, zeigt sich, dass diese unterschiedlich verläuft (vgl. Abb. 4.7). Während die Absatzzahlen von Smartphones stetig steigen, sind sie bei Notebook und Desktop-PC eher stabil und bei E-Reader und Tablet-PC tendenziell rückläufig. E-Reader werden speziell zum Lesen von E-Books entwickelt und weisen ein niedrigeres Absatzniveau als Tablet-PCs auf, die sich wie Smartphone, Laptop und Desktop-PC für unterschiedliche Zwecke eignen.

${ }^{151}$ Vgl. Dahinden/ Trappel (2010), S. 439.

${ }^{152}$ Vgl. Hermann/ Lulei (2013), S. 9; vgl. Gerlach (2014), S. 54, S. 64.

${ }^{153}$ Vgl. Roth (2009), S. 66 f.

${ }^{154}$ Vgl. Hermann/ Lulei (2013), S. 9; vgl. Gerlach (2014), S. 54, S. 64.

${ }^{155}$ Janneck et al. (2013), S. 103. 


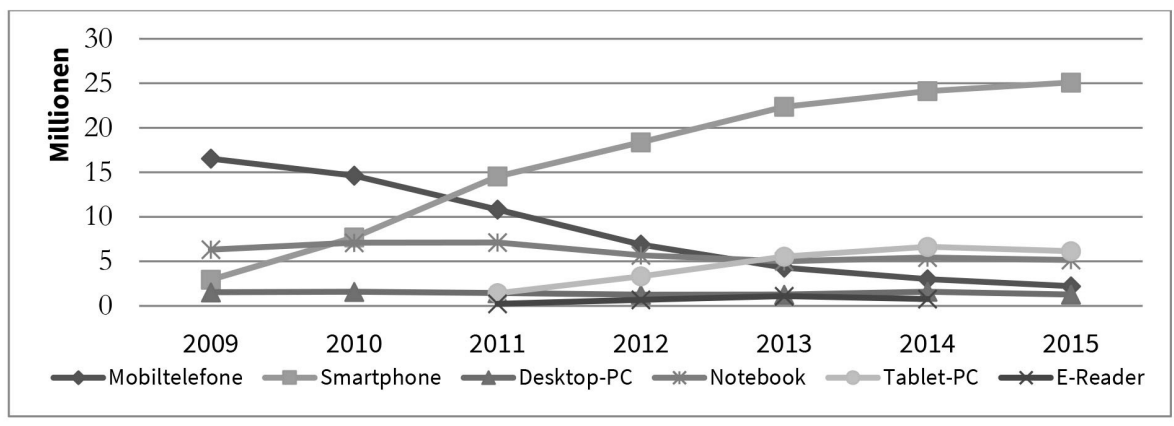

Abb. 4.7 Entwicklung der Absatzzahlen verschiedener Gerätetypen in Deutschland, in Stück (Quelle: Eigene Erstellung nach gfu et al. (2010), S. 3;gfu et al. (2012), S. 3; gfu et al. (2014), S. 3 f.; gfu et al. (2015), S. 3 f.; E-Reader Bitkom (2013), S. 2; Bitkom (2015), S. 7)

Das Smartphone nimmt als „Begleiter in allen Lebenslagen“ ${ }^{156}$ eine Sonderstellung ein, weil es durch Computertechnologie und Handlichkeit jederzeit eine Vielfalt an Funktionen bietet und eine Person es immer bei sich trägt. ${ }^{157}$ Je nach Modell sind EReader ebenfalls internetfähig und können über ein Betriebssystem verfügen, sodass Apps installierbar sind. Bei verkaufsstarken EReadern wie Tolino und Kindle ist die Installation von externen Programmen durch Kunden nicht vorgesehen. ${ }^{158}$ Beim Tolino können jedoch E-Book-Dateien mittels USB-Kabel von einem anderen Gerät dorthin übertragen oder direkt von einem Angebot auf das Gerät heruntergeladen werden. ${ }^{159}$ Die Haushaltsabdeckung mit Tablet-PCs liegt mit $35 \%$ höher als bei EReadern (15\%). In der jungen Altersgruppe 14 bis 29 Jahre befindet sich in $54 \%$ der Haushalte ein Tablet-PC und in $19 \%$ ein E-Reader. Bei Smartphones liegt mit $98 \%$ eine nahezu vollständige Haushaltsabdeckung vor (Bevölkerungsdurchschnitt 61 \%) (vgl. Abb. 4.8).

\footnotetext{
156 Schey/ Rieder (2014), S. 189.

157 Vgl. Schey/ Rieder (2014), S. 199 f.

158 Vgl. Tolino zieht am Kindle vorbei (2014), buchreport.de; vgl. Chalid El-Heliebi, 29. 09. 2015.

159 Vgl. Chalid El-Heliebi, 29. 09. 2015.
} 


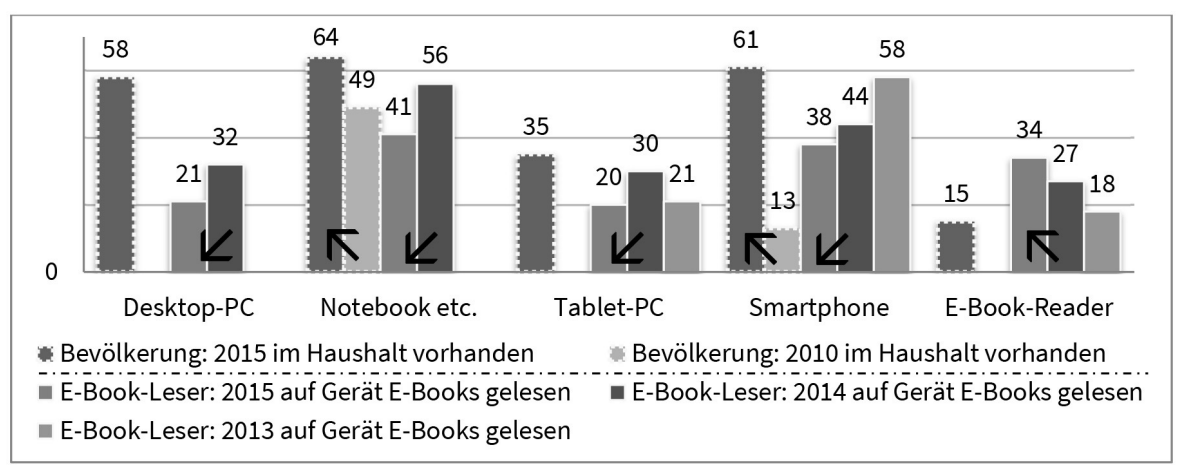

Abb. 4.8 Ausstattung deutscher Haushalte mit verschiedenen Gerätetypen, in\% (Quelle: Eigene Erstellung nach Engel/ Breunig (2015), S. 311; van Eimeren/ Ridder (2011), S. 3; für E-Book-Leser vgl. Bitkom (2013), S. 7; Bitkom (2014), S. 4; Bitkom (2015), S. 6; fehlende Werte ohne Befunde in der Literatur)

Wenn es um den Geräteeinsatz beim Bücherlesen geht, lässt sich festhalten, dass die Verteilung seit 2013 konstant ist: E-Reader haben im Vergleich zu Tablet-PC und Smartphone an Bedeutung gewonnen, jedoch sind Notebook und Smartphone absolut gesehen die am häufigsten verwendeten Geräte. Da Anbieter von Web-Clouds entscheiden, mit welchen Geräten und Betriebssystemen auf die Bücher zugegriffen werden kann, besteht das Risiko von Kompatibilitätsproblemen, wenn Personen Geräte oder Betriebssysteme nutzen, die der Anbieter nicht unterstützt.

\section{Merkmal einer Dimension der Bereitstellungsqualität: Kompatibilitätsprobleme}

Eine Person kann verschiedene Geräte parallel zum E-Book-Lesen nutzen. $17 \%$ (2014: 20 \%) der E-Book-Leser geben an dies zu tun, während 78 \% (2014: 76 \%) angeben, nur auf einem Gerät EBooks zu lesen. ${ }^{160}$ Werden mehrere Geräte zum Lesen genutzt, ist es für Kunden relevant, ob sie mit unterschiedlichen Geräten auf Bücher zugreifen können.

Merkmal einer Dimension der Bereitstellungsqualität: Zugriff auf das Kundenkonto mit mehr als einem Gerät

Ein weiteres Qualitätsmerkmal kann sein, ob der Anbieter die Eigenschaften der Geräte berücksichtigt, die der Kunde benutzt. Dazu gehören Speicherkapazität und Rechenleistung, die insbesondere bei mobilen Geräten wie Smartphone und TabletPC begrenzt sind. ${ }^{161}$ Da akkubetriebene Geräte je nach Stromverbrauch eine unterschiedlich lange Betriebszeit aufweisen, ist es vorteilhaft, wenn ein Angebot längere Zeit ohne neue Ladung genutzt werden kann.

\footnotetext{
${ }^{160}$ Vgl. Bitkom (2015), S. 9; Vgl. Bitkom (2014), S. 6.

${ }^{161}$ Vgl. Römer/ Freitag (2004), S. 660 f.
} 
Merkmal einer Dimension der Bereitstellungsqualität: Geräteeigenschaften

\subsubsection{Softwarebasierte Benutzungsschnittstelle zum Bücherlesen}

Während der Gerätebedarf für die Nutzung von E-Books offensichtlich ist, wird die Software bisher mit weniger Wichtigkeit behandelt. ${ }^{162}$ Der Zugang zum Buch ist jedoch nur über Software und eine Benutzungsoberfläche möglich. Eine Benutzungsschnittstelle wird als ein „Eigenschaftsbündel aus funktionalen und ästhetischen Merkmalen “163 definiert. Software ermöglicht in Kombination mit einer Bildschirmtechnologie die Darstellung der Bücher und stellt Funktionen bereit, für deren Nutzung technische Mittel der Bedienung erforderlich sind, wenn es sich nicht um einen Touchscreen handelt. ${ }^{164}$ Sie ist ein Werkzeug, das durch Programmierarbeit entsteht, das für einen bestimmten Zweck erstellt wird und das verschiedene Funktionen ausführen und sie auf verschiedene Daten anwenden kann. ${ }^{165}$ Die Daten verlieren beim Einsatz im Computer ihren Zustandscharakter und werden Teil von Prozessen in Form von Algorithmen, weil der Computer die Daten verarbeitet und den Output verändert. ${ }^{166}$ Schmidt weist darauf hin, dass es von den programmierten Algorithmen abhängt, welche Handlungen und Operationen möglich sind und welche nicht. ${ }^{167}$ Der Wert der Lesesoftware aus Kundensicht bemisst sich u. a. daran, welche Funktionen bereitgestellt werden. Das Urteil, welche Funktionen nötig sind oder gewünscht werden, hängt von den Lesezielen ab und davon, welche Funktionen vom Lesen gedruckter Bücher her bekannt sind und als nützlich empfunden werden (vgl. Kap. 6.2.1).

\subsection{Bereitstellungsdimension: Kosten des Zugangs}

\subsubsection{Finanzielle Kosten}

Bei der Gestaltung der Bereitstellungskosten treffen Anbieter eine Entscheidung darüber, aus welchen Quellen die zu erzielenden Erlöse stammen sollen. ${ }^{168}$ Die Erlösquellen lassen sich danach unterscheiden, welche Leistung abgerechnet wird. ${ }^{169}$ Als Gegenleistung für Werbeeinnahmen bietet der Anbieter eine Kontakt-

\footnotetext{
${ }^{162}$ Vgl. Vassiliou/ Rowley (2008), S. 362.

163 Vgl. Hagenhoff/ Kuhn (2014), S. 223.

${ }^{164}$ Vgl. Kuhn/ Hagenhoff (2015), S. 318 f.

165 Vgl. Hagenhoff (2014), S. 5, S. 12 f; vgl. Hofacker et al. (2007), S. 6; vgl. Schmidt (2011), S. 66.

166 Vgl. Rieder (2004), S. 5 ff.; vgl. Neuberger (2009), S. 26.

167 Vgl. Schmidt (2011), S. 64.

168 Vgl. Skiera/ Lambrecht (2007), S. 872.

169 Vgl. Skiera/ Lambrecht (2007), S. 876.
} 
möglichkeit zu seinen Kunden. ${ }^{170}$ Als Gegenleistung für Kundeneinnahmen bietet der Anbieter Zugang zu Büchern. Ein Sonderfall sind Bibliotheken, da diese überwiegend von öffentlichen Trägern (meist von den Kommunen) betrieben und von diesen finanziert werden. ${ }^{171}$ Als Gründe, warum trotzdem Gebühren erhoben werden, nennen die befragten Bibliotheken eine angespannte Haushaltslage, Vorgaben zur Kostendeckung oder Kosten als symbolischer Akt, da auch andere Kultureinrichtungen nicht kostenlos zugänglich sind. ${ }^{172}$ Für Kunden ist der Preisvorteil gegenüber dem Kauf der wichtigste Grund für die Leihe. ${ }^{173}$

\section{Merkmal einer Dimension der Vergleichsprozesse: Sparpotenzial eines Angebots}

Unabhängig von der Angebotsform lassen sich Kundenerlöse danach unterscheiden, ob sie bei einzelnen Transaktionen entstehen oder unabhängig davon zustande kommen. ${ }^{174}$ Transaktion meint die Übertragung im rechtlichen Sinne oder die physische Übergabe. ${ }^{175}$ Bei den transaktionsabhängigen Erlösen erfolgt die Abrechnung der Bücher nach Menge (z. B. pro Stück). ${ }^{176}$ Bei nicht-transaktionsabhängigen Erlösen wird dem Kunden einmalig oder regelmäßig wiederkehrend (Abonnement) ein fester Betrag in Rechnung gestellt und zwar unabhängig von der Anzahl an Büchern, auf die der Kunde zugreift. ${ }^{177}$ Ist in einem festgelegten Zeitraum der Zugriff auf alle Bücher, d. h. ohne Beschränkungen möglich, spricht man von einer Flatrate. ${ }^{178}$

Bei gedruckten Büchern ist es üblich, diese an andere Personen im Bekanntenund Freundeskreis auszuleihen bzw. mit Familienmitgliedern zu teilen. Dieses Verhalten ist rechtlich nicht problematisch. Allerdings fällt auch in Bibliotheken die Anzahl der Ausweise bzw. Kunden geringer aus als die Anzahl an Personen, welche das Angebot in Anspruch nehmen. ${ }^{179}$ Personen nutzen also den Zugang einer anderen Person, um Gebühren zu umgehen. ${ }^{180}$ Auch beim Flatrate-Tarif neigen Kunden dazu, ihren Zugang mit anderen Personen zu teilen. ${ }^{181}$ Zech spricht aus rechtlicher Sicht vom Problem der Leistungserschleichung. ${ }^{182}$ Darüber hinaus führt dieses Verhalten dazu, dass sich die Nutzungsintensität pro Konto bzw. Mitglied erhöht. ${ }^{183}$ Bei Werbeeinnahmen als Erlösquelle wird dieser Umstand sogar positiv

\footnotetext{
170 Vgl. ebd.

171 Vgl. Plassmann/ Syré (2004), S. 20 f.; vgl. Seefeldt (2011).

172 Vgl. Hüttemann (2010), S. 46.

${ }^{173}$ Vgl. Blanck (2006), S. 78.

174 Vgl. Wirtz (2013), S. 97 f.

175 Vgl. Reith (2007), S. 37.

176 Vgl. Wirtz (2013), S. 97 f.

177 Vgl. Wirtz (2013), S. 98; vgl. Graef (2016), S. 39.

178 Vgl. Robbert/ Roth (2011), S. 150; vgl. Gassmann/ Frankenberger/ Csik (2013), S. 125.

179 Vgl. Hüttemann (2010), S. 46.

180 Vgl. Hüttemann (2010), S. 22; vgl. Locher (2003), S. 640.

181 Vgl. Shatzkin (2014); vgl. Mander (2015)

182 Vgl. Zech (2014).

183 Vgl. Shatzkin (2014).
} 
hervorgehoben (z. B. Kennzahl LpA), da die Kontaktreichweite für Werbungtreibende eine wichtige Rolle spielt. ${ }^{184}$ Im empirischen Teil wird geprüft, ob Kunden die Möglichkeit nutzen, ihren Zugang mit anderen zu teilen.

Merkmal einer Dimension von Bereitstellungsqualität: Freiheit sich den Zugang zu teilen

Aussagen über die Auswirkungen einer unbegrenzten Nutzungsmöglichkeit und der gesteigerten Nutzungsintensität durch das Teilen von Zugängen sind gerade dann von Relevanz, wenn dem Anbieter bei jedem gelesenen Buch Kosten entstehen. ${ }^{185}$ Ein Vergütungsmodel, das trotz Flatrate-Tarif (gedeckelte Kundeneinnahmen) vorsieht, dass jedes geöffnete und teilweise gelesene Buch prozentual zum Nettoverkaufspreis abgerechnet wird, statt einen festen Prozentsatz der Gesamteinnahmen auf die teilnehmenden Verlage und Autoren umzulegen, läuft Gefahr, in finanzielle Schwierigkeiten zu geraten. ${ }^{186}$ Anbieter mit diesem Vergütungs-/Tarif-Mix setzen auf natürliche oder technische Restriktionen, welche die Nutzung einschränken z. B. fehlende Zeit oder fehlende Netzverbindung beim Abrufprinzip ${ }^{187}$ (vgl. Kap. 4.3.2) sowie auf ein ausgeglichenes Verhältnis von Viel- und Weniglesern. ${ }^{188}$ Zusätzlich können Anbieter die Freiheit bei der Nutzung einschränken. Die Akzeptanz solcher Beschränkungen stellt aus Kundensicht ein Opfer dar, weil sie die Gestaltungsfreiheit beim Bücherlesen einschränken. Um eine eher weiche Form der Beschränkung handelt es sich, wenn Anbieter z. B. festlegen, wie viele Bücher maximal gleichzeitig gelesen werden können, wenn der unbeschränkte Zugriff nur für bestimmte Bucharten gilt oder wenn Anbieter den Zeitraum pro Buch vorgeben, in dem ein Zugriff möglich ist. In diesen Fällen überwiegt noch der Flatrate-Charakter eines Angebots. Eine eher harte Form der Beschränkung stellt hingegen eine Begrenzung der absoluten Buchlesemenge in einem Zeitraum dar. „Hierdurch wird die Idee des unlimitierten Konsums (.) verwässert“. ${ }^{189}$

Merkmale einer Dimension von Bereitstellungsqualität: Freiheit Buchlesemenge, Freiheit Lesezeitraum

Es gibt verschiedene Faktoren, die einen Einfluss darauf haben, ob Kunden sich für eine Abrechnung pro Buch oder einen Pauschaltarif entscheiden und ob sie den Kauf einem temporären Erwerb vorziehen. Zum einen wägen Kunden ab, ob sie ein Buch wiederholt lesen werden. Der zweitwichtigste Grund für die Nutzung der Leihe ist für Bibliothekskunden die Erwartung, ein Buch nur einmal zu lesen. ${ }^{190}$ Dies gilt

\footnotetext{
${ }^{184}$ Vgl. Brosius (2013), S. 186.

185 Vgl. Robbert/ Roth (2011), S. 150.

${ }^{186}$ Vgl. Shatzkin (2014); vgl. Lenz (2014)/ Buchreport, S. 64; vgl. Ausgelesen (2016), buchreport.de.; Flatrate unter Druck (2016), buchreport.de.

187 Vgl. Wirtz (2013), S. 98.

188 Vgl. Gassmann/ Frankenberger/ Csik (2013), S. 125; vgl. Lenz (2014)/ Buchreport, S. 64 f.

189 Gassmann/ Frankenberger/ Csik (2013), S. 125.

${ }^{190}$ Vgl. Blanck, (2006) S. 78.
} 
analog für die Angebotsform Miete: Je häufiger eine Person erwartet, einen Film anzuschauen, desto wahrscheinlicher ist der Kauf gegenüber der Miete. ${ }^{191}$ Außerdem überlegen Kunden, wie viel sie in einem bestimmten Zeitraum z. B. im Monat lesen. Der Einfluss der antizipierten Nutzungshäufigkeit auf die Entscheidung für einen Tarif wurde auch empirisch bestätigt. ${ }^{192}$ Allgemein fällt die finanzielle Belastung je nach Abrechnungsmodus unterschiedlich hoch aus, d. h. es entstehen besonders betroffene Nutzergruppen. ${ }^{193}$ Während ein Pauschaltarif Vielnutzer begünstigt, profitieren Wenignutzer von einer Abrechnung pro Titel. Demnach wägen Kunden vermutlich ab, ob sich das Angebot bei einem bestimmten Tarif gemessen an der eigenen Leseintensität finanziell lohnt.

Merkmal einer Dimension der Vergleichsprozesse: Kosten-Buchlesemengen-Verhältnis

Daneben kann die Tarifoption Flatrate selbst einen spezifischen Wert für die Kunden haben. ${ }^{194}$ In diesem Fall wird auch von einem Flatrate-Bias gesprochen, d. h. Kunden bezahlen einen Pauschalpreis, obwohl die Kosten gemessen an der Buchlesemenge bei einer Abrechnung pro Stück geringer ausfallen würden. ${ }^{195}$ Dieser Effekt wurde häufig bei Tarifentscheidungen für Telefon- oder Internetnutzung untersucht ${ }^{196}$, jedoch existieren bisher keine Studien zur Tarifwahl bei Buchangeboten. ${ }^{197}$ Allgemein ist es wahrscheinlicher, dass ein Flatrate-Bias vorliegt, wenn Kunden ihre Nutzungsmenge überschätzen. ${ }^{198}$ Einen Taxametereffekt - gemäß dem Kunden einen Flatrate-Tarif schätzen, weil sie bei der Nutzung nicht über die Kosten nachdenken müssen - kann Robbert bei Kunden eines Fitnessstudios mit Langzeitvertrag nicht nachweisen. ${ }^{199}$ Bei der Untersuchung von Bibliotheken konnte jedoch empirisch bestätigt werden, dass Kunden bei der Abrechnung pro Stück ihren Konsum aufgrund der steigenden Kosten einschränken. ${ }^{200}$ Es wird daher angenommen, dass Kunden beim Flatrate-Tarif oder einer kostenlosen Bereitstellung die gedankliche Entkopplung des Bücherlesens von finanziellen Kosten schätzen. Andere Studien konnten den Effekt als Einflussfaktor auf die Wahl der Angebotsform (Kaufpräferenz) ${ }^{201}$ bzw. auf die Einstellung gegenüber einem Angebot mit Flatrate-Tarifoption ${ }^{202}$ nachweisen.

\footnotetext{
191 Vgl. Mann (2010), S. 148 f., S. 183 f., S. 206.

192 Vgl. Robbert (2013), S. 172.

193 Vgl. Hüttemann (2010), S. 23.

194 Vgl. Lambrecht/ Skiera (2005), S. 615.

195 Vgl. Robbert (2013), S. 2; vgl. Lambrecht/ Skiera (2005), S. 598.

196 Vgl. Robbert (2013), S. 2.

197 Vgl. Tesar (2013), S. 31.

198 Vgl. Robbert (2013), S. 161, S. 165; vgl. Lambrecht/ Skiera (2005), S. 606, S. 612.

199 Vgl. Robbert (2013), S. 161, S. 165.

200 Vgl. Hüttemann (2010), S. 26 f., S. 60.

201 Vgl. Mann (2010), S. 185 f., S. 202, S. 204.

202 Vgl. Dörr et al. (2013), S. 21.
} 
Merkmal einer Dimension von Bereitstellungsqualität: Entkopplung von Kosten und Lektüre

Robbert weist darauf hin, dass sich die Gründe für die Wahl eines Tarifs bei Produkten unterscheiden können z. B. gibt es Angebote, bei denen es den Kunden darum geht, die Kosten der Nutzung zu limitieren, während sie bei anderen Angeboten die Nutzungsintensität steigern möchten. ${ }^{203}$ Bei letzteren kann Robbert am Beispiel eines Fitnessstudios einen Precommitmenteffekt bzw. Selbstdisziplinierungseffekt nachweisen. ${ }^{204}$ Hierbei geht es Kunden darum, sich durch den Abschluss eines Langzeitvertrags bzw. durch finanzielle Ausgaben im Vorfeld zu einer regelmäßigen Angebotsnutzung zu motivieren. Der freiwillig erzeugte Kostendruck dient dem Kunden als Instrument, um in einer Entscheidungssituation die Wahrscheinlichkeit zu erhöhen, dass sie sich für eine bestimmte Option (z. B. die Tätigkeit Bücherlesen) entscheiden. Robbert merkt an, dass die Idee des Kunden, finanzielle Ausgaben als Mittel der Selbstkontrolle einzusetzen, auch bei kulturellen Angeboten wie Theater- und Bücherabonnements eine Rolle spielen könnte. ${ }^{205}$ Auch geben knapp 40 \% der Abonnenten von Publikumszeitschriften als Vorteil eines Abonnements an: „Man liest eine Zeitschrift eher, wenn man sie im Abo hat, weil man sie nicht ungelesen wegwerfen will.“206 Daher soll geprüft werden, ob Kunden finanzielle Ausgaben in Verbindung mit einem Abonnement zur Selbstdisziplinierung einsetzen.

Merkmal einer Dimension von Bereitstellungsqualität: Möglichkeit der Selbstdisziplinierung

\subsubsection{Fehlende Transparenz der Benutzungsschnittstelle zum Bücherlesen}

\subsubsection{Unterbrechung der Transparenz durch Störungen}

Eine transparente Benutzungsschnittstelle bedeutet wenig Ablenkung von einer Aufgabe. ${ }^{207}$ Transparenz unterstützt das Involvement des Benutzers: „Je transparenter der Textzugang wahrgenommen wird (...) desto größer wird die Einbindung des Rezipienten in den vermittelten Inhalt: die Benutzungsschnittstelle ,verschwindet" während der Nutzung aus der bewussten Wahrnehmung. “208 Um die Bedeutung der Transparenz verstehen zu können, ist es notwendig, die inhaltlichen Stimuli im Zuge der Bedeutungskonstruktion (Buch) und die Reize der Benut-

\footnotetext{
203 Vgl. Robbert (2013), S. 106.

${ }^{204}$ Vgl. Robbert (2013), S. 161, S. 165, S. 172, S. 186.

205 Vgl. Robbert (2013), S. 186.

206 Jagow (2012), S. 26.

207 Vgl. Kaptelinin/ Nardi (2006), S. 62 f.

208 Hagenhoff/ Kuhn (2014), S. 226.
} 
zungsschnittstelle zu unterscheiden. ${ }^{209} \mathrm{Zu}$ einer Unterbrechung der Transparenz kommt es durch Störungen. Störungen sind externe, d. h. durch die Benutzungsschnittstelle ausgelöste psychologische Reize, die in jedem Fall kognitiven Aufwand verursachen. ${ }^{210}$ Störungen sind konkret Farben, Geräusche und Bewegung sowie in virtuellen Angeboten eingeblendete Funktionen und Werkzeuge einer Anwendung, aber auch eine eingeblendete Zugriffsleiste, um zu anderen Anwendungen zu gelangen, oder um eingeblendete Statusmeldungen anderer Anwendungen. ${ }^{211}$ Der Zugriffslink auf andere Anwendungen kann je nach Leseziel als nützlich empfunden werden. ${ }^{212}$ Störungen werden vom Kunden also nicht in jedem Fall als Kosten wahrgenommen. Andererseits befürchten Untersuchte beim Lesen auf Tablet-PCs im Gegensatz zum Lesen von gedruckten Büchern und auf E-Readern - durch andere Anwendungen wie Internet, E-Mail oder Spiele abgelenkt zu werden. ${ }^{213}$ Folgen von Störungen sind Aufmerksamkeitslenkung auf bestimmte Punkte (fokussierte Aufmerksamkeit) ${ }^{214}$ sowie kognitive oder emotionale Reaktionen. Negativ ist z. B. das Gefühl von Kontrollverlust und positiv das Gefühl (angenehmer) Überraschung. ${ }^{215}$

\subsubsection{Werbung in Büchern als spezielle Form der Störung}

Eine Störung stellt auch Werbung dar, wenn sie gemeinsam mit dem Buchinhalt angezeigt wird.

Merkmal einer Dimension von Bereitstellungsqualität: Transparenz der Benutzungsschnittstelle bei Werbung

Wie stark kognitiv belastend ein Werbemittel ist, hängt vermutlich von der Werbeform ab. Je nach Gestaltung lösen Werbemittel als äußere Stimuli Orientierungsreflexe aus z. B. durch Helligkeit, Farbe und Lautstärke. ${ }^{216}$ In virtuellen Angeboten kann sich das Werbemittel über die Seite legen (Pop-Up-Werbung) und es entsteht auf jeden Fall ein Werbekontakt. ${ }^{217}$ Pop-Up-Werbung und Werbung mit Geräuschen und Animationen werden in dieser Arbeit als reizstarke Werbemittel aufgefasst und von statischer Bannerwerbung (reizarm) abgegrenzt. Im Gegensatz zu Pop-UpFenstern lösen statische Banner keinen Orientierungsreflex aus. ${ }^{218}$ Unter einem Orientierungsreflex versteht man orientierende Aufmerksamkeit, also die

\footnotetext{
209 Vgl. Hagenhoff/ Kuhn (2014), S. 224.

${ }^{210}$ Vgl. Hagenhoff/ Kuhn (2014), S. 237.

211 Vgl. Hagenhoff/ Kuhn (2014), S. 225.

${ }^{212}$ Vgl. Henke (2002), S. 11.

${ }^{213}$ Vgl. Janneck et al. (2013), S. 103.

${ }^{214}$ Vgl. Hagenhoff/ Kuhn (2014), S. 225 f.; vgl. auch Foscht/ Swoboda/ Schramm-Klein (2015), S. 40.

215 Vgl. Hagenhoff/ Kuhn (2014), S. 237.

${ }^{216}$ Vgl. Foscht/ Swoboda/ Schramm-Klein (2015), S. 41.

${ }^{217}$ Vgl. Naab/ Schlütz (2016), S. 234; vgl. Klimmt/ Weinacht/ Donner (2009), S. 14.

${ }^{218}$ Vgl. Diao/ Sundar (2004), S. 554 f.
} 
Aufmerksamkeitslenkung im Raum. ${ }^{219}$ Aufgrund der geringeren Aufmerksamkeit für das Banner-Werbemittel fallen daher auch die Erinnerungswerte signifikant geringer aus als bei Pop-Up-Fenstern. ${ }^{220}$ Die Kontaktchance und die Erinnerungsleistung sind wichtige Kriterien für Werbungtreibende, da z. B. der Kontakt die Voraussetzung für eine Wirkung ist. ${ }^{221}$ Allerdings wird Pop-Up-Werbung bei einer zielgerichteten Aufgabe als in negativer Weise störend empfunden. ${ }^{22}$ Auch Bücherlesen kann als eine zielgerichtete Aufgabe aufgefasst werden. Die Werbevermeidungsstrategie bei gedruckten Zeitungen und Zeitschriften - die Seiten mit Werbung einfach zu überblättern ${ }^{223}$ - ist bei Büchern eher ungeeignet, wenn diese linear gelesen werden und ein Überspringen von Seiten das Textverständnis negativ beeinflusst. In virtuellen Angeboten können die Betreiber die Bannerwerbung zudem über mehrere Seitenwechsel aktiviert halten. Einer Person bleibt daher nur der Versuch, sich stärker den inneren Reizen zuzuwenden, die infolge von Vorstellbildern während des Lesens entstehen, um die Werbewirkung zu vermeiden. ${ }^{224}$ Insgesamt kann Werbung einerseits eine aktive Zuwendung erfahren, wenn sie als informativ oder unterhaltsam bewertet wird. ${ }^{225}$ Andererseits kann sie aber auch die mentale Verarbeitung des Inhalts stören oder die Lesetätigkeit unterbrechen.

Im empirischen Teil der Arbeit wird nach Anhaltspunkten gesucht, ob Leser Werbeeinblendungen als belastend empfinden, ob das Ausmaß der Störung von der Art des Werbemittels beeinflusst wird (reizstark oder reizarm) und ob Kunden Werbevermeidungsstrategien einsetzen.

\subsubsection{Folgekosten einer Fehlentscheidung}

Kunden bewerten im Vorfeld, wie leicht sie eine nicht-optimale Auswahlentscheidung (Buch gefällt nicht) rückgängig machen können. Die Frage betrifft die Reversibilität einer Entscheidung, worunter das Ausmaß verstanden wird, ,in dem ein Nutzer Fehler oder Entscheidungen, die sich nachträglich als unpassend oder unerwünscht erweisen, wieder rückgängig machen kann. “226 Hartmann spricht von Reversibilitätskosten als Kosten, die in Folge einer Fehlentscheidung entstehen ${ }^{27}$ und Jäckel von den Folgekosten, deren Höhe eine Person in einer Entscheidungssituation festlegt. ${ }^{228}$ Folgekosten können sich in Form von Zeit, Mühe und mone-

\footnotetext{
${ }^{219}$ Vgl. Foscht/ Swoboda/ Schramm-Klein (2015), S. 40.

${ }^{220}$ Vgl. Klimmt/ Weinacht/ Donner (2009), S. 13; vgl. Diao/ Sundar (2004), S. 555.

221 Vgl. Naab/ Schlütz (2016), S. 223 f.; vgl. Foscht/ Swoboda/ Schramm-Klein (2015), S. 41.

${ }^{222}$ Kohler (2007), S. 94.

${ }^{223}$ Vgl. Naab/ Schlütz (2016), S. 231.

${ }^{224}$ Vgl. Foscht/ Swoboda/ Schramm-Klein (2015), S. 41.

${ }^{225}$ Vgl. Naab/ Schlütz (2016), S. 232.

${ }^{226}$ Wirth/ Schweiger (1999), S. 59.

227 Vgl. Hartmann (2006), S. 55.

${ }^{228}$ Vgl. Jäckel (2011), S. 94, S. 375; vgl. Jäckel (1992), S. 254.
} 
tären Kosten ausdrücken. Allgemein sind Personen durch die Internetnutzung gewöhnt, dass eine hohe Reversibilität durch die Navigationshistorie und die ZurückFunktion gegeben ist. ${ }^{229}$ Im Hinblick auf die monetären Folgekosten wiegen diese bei einer transaktionsabhängigen Abrechnung schwerer als bei der Nutzung von kostenlosen Web-Angeboten und beim Fernsehen ${ }^{230}$ sowie vermutlich insgesamt beim Flatrate-Tarif. Im Handel sind Buchrückgabe und Kostenerstattung mit Aufwand verbunden. Zudem besteht kein Rechtsanspruch auf den Umtausch, allerdings sind Händler häufig sehr kulant. Riethmüller (Geschäftsführer Osiander) weist darauf hin, dass Kunden teilweise aufgrund der leichten Umtauschbedingungen bei Osiander einkaufen. ${ }^{231}$ Im Versandhandel (BringPrinzip, vgl. Kap. 4.3.1) muss das Buch zurückgeschickt werden, was ebenfalls Aufwand und ggf. monetäre Kosten verursacht ebenso wie der Wiederverkauf eines Buches auf dem Gebrauchtmarkt, der zudem nur bei gedruckten Büchern möglich ist ${ }^{232}$ und bei dem die Erlöse meist unter dem gezahlten Kaufpreis liegen. ${ }^{233}$ Dem Miet- und Leihmodell ist die Reversibilität hingegen inhärent. Allerdings kann der früheste Rückgabezeitpunkt vertraglich und/oder technisch festgelegt sein. Zum Beispiel war beim Angebot „Onleihe“ bis August 2015 die E-Book-Rückgabe technisch so implementiert, dass Kunden E-Books bis zum Leihfristende behalten mussten. ${ }^{234} \mathrm{Ob}$ dies problematisch ist, hängt davon ab, wie viele Medien ein Kunde gemäß Vertrag/Satzung parallel ausleihen kann. Ist das Ausleihkontingent einer Person ausgeschöpft, wenn sie feststellt, dass ihr die ausgeliehenen Bücher nicht gefallen, wiegen die Folgekosten schwerer, da zunächst keine neue Ausleihe möglich ist. Kunden schätzen daher vermutlich das Gefühl, sich keine Gedanken über die Folgen einer Fehlentscheidung machen zu müssen.

Merkmal einer Dimension von Bereitstellungsqualität: Freiheit Lektüre abzubrechen wegen Nichtgefallen

\subsection{Bereitstellungsdimension: Leichtigkeit der Benutzung}

Ein häufig verwendetes Konstrukt, um die Einfachheit der Benutzung eines Systems $\mathrm{zu}$ messen ist die perceived ease of use nach Davis. ${ }^{235}$ In die Bewertung fließen insbesondere der physische Aufwand, der mentale Aufwand und der Eindruck einer Person ein, wie leicht die Benutzung erlernt werden kann. ${ }^{236}$ Ausgehend von der

\footnotetext{
${ }^{229}$ Vgl. Wirth/ Schweiger (1999), S. 59 f.; vgl. Stark/ Magin/ Jürgens (2014), S. 30.

${ }^{230}$ Vgl. Jäckel (2011), S. 379; vgl. Schweiger (2010), S. 194.

${ }^{231}$ Vgl. Graf (2014), ab 7.55 Min., https://www.youtube.com/watch?v=j9u7FD1elAA.

${ }^{232}$ Vgl. Graef (2016), S. 3.

${ }^{233}$ Vgl. Schumann (2013), S. 54.

${ }^{234}$ Vgl. Leipziger Städtische Bibliotheken (2015a).

${ }^{235}$ Vgl. Rossmann (2011), S. 77 f.

${ }^{236}$ Vgl. Davis (1989), S. 325.
} 
Interaktion mit einem System definiert Davis die Einfachheit der Benutzung als ,the degree to which a person believes that using a particular system would be free of effort“. ${ }^{237}$ Aussagen in der Realität, die diesem Konstrukt zugeordnet werden können, sind z. B. „einfach zu erlernen“, „klar und verständlich“, „einfach zu nutzen“. ${ }^{238}$ In einer physischen Umgebung wird die wahrgenommene Convenience eines Ladens positiv von der Orientierungsfreundlichkeit des Raums beeinflusst und davon, wie schnell die Regale mit den gewünschten Produkten erreicht werden können. ${ }^{239}$

Merkmal einer Dimension von Bereitstellungsqualität: Leichtigkeit der Benutzung

${ }^{237}$ Davis (1989), S. 320.

238 Vgl. Davis (1989), S. 331.

${ }^{239}$ Vgl. Reith (2007), S. 127, 147. 



\section{Kundenaktivitäten}

\subsection{Verständnis von Kundenaktivitäten in der aktuellen Forschung}

Innerhalb der Forschung zum Relationship Marketing und Management wird angemerkt, dass es bisher nur wenige Studien gibt, die sich mit dem Thema der Kundenaktivitäten aus dem Blickwinkel des Kunden selbst beschäftigen. ${ }^{240}$ Üblich ist ein Verständnis, gemäß dem Kunden lediglich bei den Prozessen des Anbieters mitwirken. Demgegenüber lässt sich der Kunde auch als Person begreifen, die hauptsächlich unabhängig vom Anbieter agiert und sich von ihren eigenen Zielen leiten lässt. ${ }^{241}$ Diese Arbeit schließt sich der zweiten Sichtweise an.

Allgemein wird Aktivität als „etwas tun“ definiert, jedoch hat dieses Vorgehen einen tautologischen Charakter. ${ }^{242}$ Mickelsson ergänzt daher die Merkmale abgrenzbare Verhaltenseinheit, Anfang und Ende - durch Menschen bestimmt - sowie einen Auslöser der Aktivität. ${ }^{243}$ Als Startereignis kann ein Bedürfnis gelten, während der Endpunkt wiederum die Bedürfnisbefriedigung darstellt. ${ }^{244}$ Begrifflich lässt sich die Aktivität einer Person im Sinne von „aktiv sein“ von den Aktivitäten abgrenzen, d. h. den Verhaltenseinheiten mit definierbarem Anfang und Ende. ${ }^{245}$ Nach Reith sind Einkaufsaktivitäten mit Kosten in Form von Zeit und Mühe verbunden. ${ }^{246}$ Unter Mühe fasst sie physische Kosten sowie emotionale und kognitive (psychische) Kosten zusammen. ${ }^{247}$ Jeder Kunde erlebt die Kosten einer Aktivität bezogen auf einen Referenzpunkt, d.h. es geht nicht um eine objektive Kostenhöhe, sondern darum, in welchem Maße sich das aktiv sein anstrengend anfühlt. ${ }^{248}$ Statt von Kosten kann auch von Opfern gesprochen werden, die mit den Aktivitäten verbunden sind. ${ }^{249}$ Fließ et al. kommen in einer qualitativen Studie zu dem Schluss, dass Kunden bei Dienstleistungen (Fahrtkarte kaufen, Zahnarztbesuch etc.) sowohl physisch als auch mental aktiv sind und somit Aufwand investieren. Zur physischen Aktivität gehören die sensorische Wahrnehmung, Gespräche, Bewegungen (sich

\footnotetext{
${ }^{240}$ Vgl. Fließ et al. (2015), S. 187 f.; vgl. Mickelsson (2014), S. 10.

${ }^{241}$ Vgl. Mickelsson (2014), S. 16.

${ }^{242}$ Vgl. Mickelsson (2014), S. 14.

${ }^{243}$ Vgl. Mickelsson, S. 44.

${ }^{244}$ Vgl. Hörstrup (2012), S. 97; Möglich ist, dass sich keine Befriedigung einstellt und die Aktivität abgebrochen wird.

245 Vgl. Mickelsson (2014), S. 27.

246 Vgl. Reith (2007), S. 40.

247 Vgl. Reith (2007), S. 32 f.

${ }^{248}$ Mickelsson (2014), S. 138

${ }^{249}$ Vgl. Mickelsson (2014), S. 140; vgl. Heinonen (2010), S. 6.
} 
selbst bewegen, Objekte bewegen) und anwesend sein. ${ }^{250}$ Die mentale Aktivität ist Voraussetzung für physische Aktivitäten ${ }^{251}$ und beinhaltet z. B. sich an etwas erinnern, Informationen suchen und verarbeiten und Entscheidungen treffen. ${ }^{252}$ Emotionen treten als Begleiterscheinung von physischen und psychischen Aktivitäten auf und stehen in einer direkten Beziehung zu diesen. ${ }^{253}$ Sie haben einen informativen Wert, da sie sich - anders als die Stimmung einer Person - auf eine konkrete Ursache bezieht. ${ }^{254}$ Innerhalb der Kommunikationswissenschaft wird der Versuch unternommen, Aktivitäten von Internetnutzern ein Aktivitätsniveau bzw. einen bestimmten Grad an Aufwand zuzuschreiben. ${ }^{255}$ Allerdings wurde dies noch nicht empirisch geprüft. Fließ et al. bestätigen bei Dienstleistungen, dass mentale Aktivität z. T. als mühevoller erlebt wird als körperliche Aktivität. ${ }^{256}$

\subsection{Verständnis von Kundenaktivitäten aus tätigkeitstheoretischer Perspektive}

\subsubsection{Grundverständnis der kulturhistorischen Schule}

Um den Begriff Aktivität konkreter fassen zu können, wird auf die Tätigkeitstheorie zurückgegriffen. Sie ist aus der Psychologie der kulturhistorischen Schule hervorgegangen und wurde besonders von A. N. Leontjew detailliert ausgearbeitet. ${ }^{257}$ Der Beginn der kulturhistorischen Schule wird auf die 1920er/ 1930er Jahre datiert und ihre Wurzeln liegen in der Sowjetunion. Mittlerweile wurde die Tätigkeitstheorie in verschiedenen Ländern und Disziplinen weiterentwickelt. ${ }^{258}$ Sie bildet u. a. einen Bezugsrahmen für Forschungsfragen in der Entwicklungspsychologie. ${ }^{259}$ Allgemein gilt der Mensch als Gestalter seiner Entwicklung, die jedoch auch vorsieht, dass er sich die kulturellen Inhalte einer Gesellschaft aneignet. ${ }^{260}$ Die Aneignung von Kultur kann jedoch nicht direkt erfolgen, sondern es ist ein Element erforderlich, dass zwischen einem Individuum und seiner Umgebung vermittelt. Dieses Element ist die Tätigkeit. ${ }^{261}$ Ein Mensch ist genau dann tätig, wenn er einen Gegenstand findet, der ihn dazu motiviert und auf die er seine Aktivität richten kann: „das konstituie-

\footnotetext{
${ }^{250}$ Vgl. Fließ/ Dyck/ Schmelter (2014), S. 444.

${ }^{251}$ Vgl. Fließ et al. (2015), S. 192.

${ }^{252}$ Vgl. Fließ/ Dyck/ Schmelter (2014), S. 446.

${ }^{253}$ Vgl. Fließ et al. (2015), S. 195; vgl. Fließ/ Dyck/ Schmelter (2014), S. 452.

254 Vgl. Werth/ Mayer (2008), S. 88.

255 Vgl. Hautzer/ Lünich/ Rössler (2012), S. 35 f.; vgl. Rudolph (2014), S. 76; Jungnickel/ Schweiger (2014), S. 32.

${ }^{256}$ Vgl. Fließ/ Dyck/ Schmelter (2014), S. 458.

257 Vgl. Rückriem (2014), S. 75 f.

258 Vgl. Kölbl (2010), S. 182 f.; vgl. Kaptelinin/ Kuutti/ Bannon (1995), S. 190.

259 Kölbl (2010), S. 191.

${ }^{260}$ Vgl. Oerter (2008), S. 92.

${ }^{261}$ Vgl. Oerter (2008), S. 97; vgl. Leontjew (1977/ 1982), S. 18, S. 40; vgl. Kaptelinin et al. (1995), S. 191.
} 
rende Merkmal der Tätigkeit ist ihre Gegenständlichkeit““ ${ }^{262}$ Die Bedürfnisse einer Person sind zwar die Voraussetzung für das tätig sein, jedoch reichen sie zur Motivation solange nicht aus, bis ein Gegenstand dazukommt, mit dem sich die Bedürfnisse befriedigen lassen. ${ }^{263}$ Dieser Gegenstand kann „sowohl stofflich als auch ideell sein, sowohl in der Wahrnehmung gegeben sein als auch nur in der Phantasie, nur in Gedanken existieren“. ${ }^{264}$ Bei kulturellen Gegenständen erlernt der Mensch erst mit der Zeit einen bestimmten Gegenstandsbezug, also die gesellschaftlich als richtig wahrgenommenen Umgangsweisen, zum Beispiel Seife beim Händewaschen benutzen oder Striche mit einem Farbstift zeichnen, sodass die Kultur ebenfalls als Bindeglied zwischen Mensch und Umwelt fungiert. ${ }^{265}$ Auch das Lesen von Büchern ist ein gelernter Gegenstandsbezug, der zusätzlich das Erlernen einer Schriftsprache erfordert. ${ }^{266}$ Nur weil die Bedürfnisse in den Beispielen gegenständlich geworden sind, können sie die Tätigkeit einer Person steuern. ${ }^{267}$ Durch die Aneignung von Objekten können Menschen zudem neue Bedürfnisse entwickeln, weil sie Wissen darüber erwerben, ob sich ein Objekt zur Bedürfnisbefriedigung eignet, sodass die Bedürfnisse reproduziert werden. ${ }^{268}$

Insgesamt lässt sich die Tätigkeitstheorie nicht als klassische Theorie anwenden, welche die Tätigkeit als Untersuchungsgegenstand hat und Erklärungsprinzipien bietet. Sie ist eher eine Methode, der Annahmen zugrunde liegen, welche die Basis für Analysen in ganz unterschiedlichen Bereichen bilden können. ${ }^{269}$ Im Folgenden werden zwei Grundprinzipien erläutert: die Makrostruktur und die Grundtypen der Tätigkeit. Ihre Anwendung bietet den Vorteil, einen Untersuchungsgegenstand systematisch analysieren zu können, statt ihn lediglich zu beschreiben. ${ }^{270}$

\subsubsection{Makrostruktur der Tätigkeit}

Oerter spricht von den drei Ebenen einer Handlung, während Leontjew sie als Makrostruktur einer Tätigkeit bezeichnet (vgl. Abb. 5.1). ${ }^{271}$

Die oben beschriebene Gerichtetheit der Tätigkeit sind Motive, die Bedürfnissen entsprechen. ${ }^{272}$ Grundlegende Motive wie das Erleben von Kontrolle, Autonomie, Verbundenheit und Selbstwirksamkeit bilden den motivationalen Rahmen. Ihre Bedeutung ergibt sich nicht nur aus der Situation heraus, sondern auf der Basis der

\footnotetext{
${ }^{262}$ Vgl. Leontjew (1977/ 1982), S. 41.

${ }^{263}$ Vgl. Leontjew (1977/ 1982), S. 42, S. 48.

264 Leontjew (1977/ 1982), S. 48.

${ }^{265}$ Vgl. Oerter (2008), S. 97 f.

${ }^{266}$ Vgl. Graf (2004), S. 9.

267 Vgl. Leontjew (1977/ 1982), S. 42.

268 Vgl. Leontjew (1977/ 1982), S. 42, S. 48; vgl. auch Kiefer (2005), S. 276.

269 Vgl. Kaptelinin/ Kuutti/ Bannon (1995), S. 191; vgl. Rückriem (2014), S. 78.

270 Vgl. Mickelsson (2014), S. 110.

271 Vgl. Oerter (1999), S. 180; vgl. Leontjew (1977/ 1982), S. 51.

${ }^{272}$ Vgl. Leontjew (1977/ 1982), S. 48.
} 

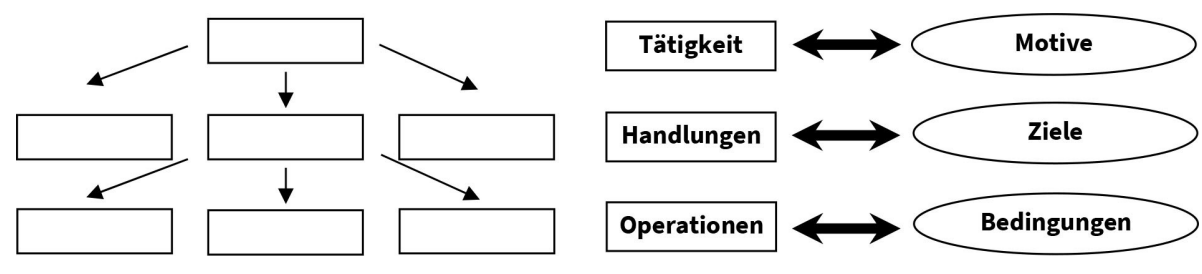

Abb. 5.1 Makrostruktur der Tätigkeit (Quelle: Eigene Erstellung nach Kaptelinin/ Nardi (2006), S. 53)

Biografie einer Person ${ }^{273}$ und immer dann, wenn ,in einer Handlung das Selbst und seine Stellung in der Welt mitbetroffen sind. “274 Die grundlegenden Motive konkretisieren sich durch Lebenserfahrungen zu spezifischeren Motiven und Interessen. ${ }^{275}$ V.a. grundlegende Motive sind einer Person nicht bewusst bzw. nicht ausdrückbar. ${ }^{276}$ Die Motive der Tätigkeit initiieren Handlungen, durch die eine Tätigkeit realisiert wird. Handlungen sind wiederum auf Ziele ausgerichtet. ${ }^{277}$ Motive beantworten somit die Frage, warum ein Ziel für eine Person bedeutsam ist. ${ }^{278}$ Die Zielsetzung (Was soll erreicht werden?) wird dadurch konkret, indem die Person die objektiven Bedingungen und verfügbaren Mittel in einer Situation bestimmt (Wie kann das Ziel erreicht werden?). ${ }^{279}$ Die Art und Weise, wie Handlungen verwirklicht werden, bezeichnet Leontjew als Verfahren, Operation oder auch Ausführungsweise einer Handlung. ${ }^{280}$ Die Ausführung erfolgt nahezu unbewusst und kann von Maschinen übernommen werden. ${ }^{281}$ Leontjew weist aber darauf hin, dass die maschinelle Operation „trotzdem Handlungen des Subjekts (realisiert)“ ${ }^{\text {(282 }}$. Zwar besitzt die Maschine Handlungsfähigkeit (Agency), weil sie Veränderungen bzw. Wirkungen in der Umwelt erzeugt, die ein Lebewesen absichtlich herbeiführen möchte. ${ }^{283}$ Allerdings ist die Agency von Artefakten eine andere als die von Lebewesen, weil sie keine eigenen Bedürfnisse haben und nicht selbst Absichten entwickeln können. ${ }^{284}$ Kaptelinin und Nardi sprechen daher von delegierter Agency. ${ }^{285}$

In Bezug auf das Verständnis der Unterteilung in Einheiten weist Leontjew zum einen auf „(d)ie Beweglichkeit der einzelnen Struktureinheiten des Tätigkeitssys-

\footnotetext{
${ }^{273}$ Vgl. Oerter (19), S. 62, S. 64; vgl. Holodynski/ Oerter (2008), S. 537; vgl. Oerter (1999), S. 182 f.

274 Oerter (1999), S. 183.

${ }^{275}$ Vgl. Holodynski/ Oerter (2008), S. 537.

276 Vgl. Oerter (1999), S. 180, S. 183.

277 Vgl. Leontjew (1977/ 1982), S. 48 f.

${ }^{278}$ Vgl. Oerter (1999), S. $180 \mathrm{f}$.

${ }^{279}$ Vgl. Leontjew (1977/ 1982), S. 50; vgl. Holodynski/ Oerter (2008), S. 538.

280 Vgl. Leontjew (1977/ 1982), S. 50.

281 Vgl. Leontjew (1977/ 1982), S. 51.

282 Leontjew (1977/ 1982), S. 51.

${ }^{283}$ Vgl. Kaptelinin/ Nardi (2006), S. 169, S. 171; vgl. auch Sundar et al. (2015), S. 65 f.

${ }^{284}$ Vgl. Kaptelinin/ Nardi (2006), S. 169, S. 175.

${ }^{285}$ Vgl. Kaptelinin/ Nardi (2006), S. 170, S. 174.
} 

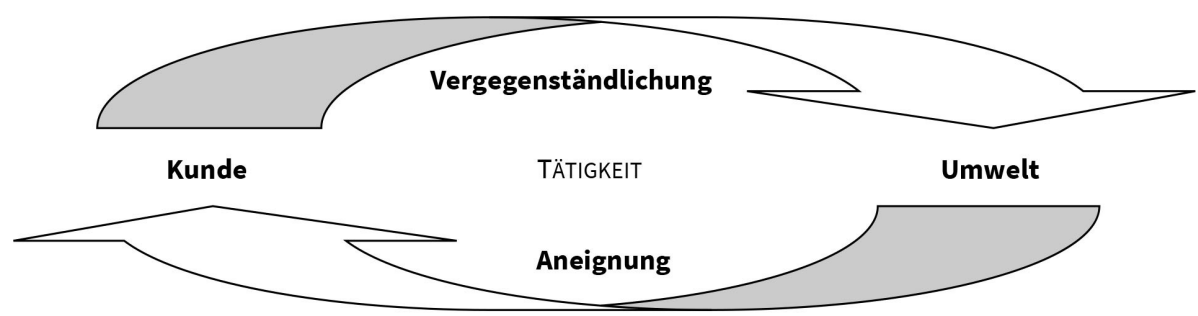

Abb. 5.2 Grundtypen der Tätigkeit (Quelle: Eigene Erstellung in Anlehnung an Oerter (2014), S. 125)

tems ${ }^{\text {‘286 }}$ hin, d. h. die definierten Einheiten sind in ihrer Anzahl nicht fix und können zu einer anderen Ebene transformiert werden, und zum anderen darauf, dass die Anwendung des Strukturprinzips ,nicht die lebendige Tätigkeit in Elemente zergliedert, sondern die charakteristischen inneren Beziehungen aufdeckt. (...) Andernfalls sind wir nicht einmal zur Lösung einfachster Aufgaben imstande, zum Beispiel darüber zu urteilen, ob in einem gegebenen Fall eine Handlung oder eine Operation vorliegt. ${ }^{287}$ Diese Hinweise aufgreifend, dominiert in der Arbeit der Begriff Aktivität, da die Zuordnung zu einer Ebene immer vom Kontext abhängt und nur bei einer Detailbetrachtung möglich ist. Wenn es um das tiefergehende Verständnis einer Aktivität geht, soll versucht werden, die Elemente in der Hierarchie anzuordnen. Die Makrostruktur wird somit nur im Bedarfsfall herangezogen.

\subsubsection{Tätigkeit als Austauschprozess zwischen Kunde und Umwelt}

Kundenaktivitäten vermitteln zwischen einem Kunden und seiner Umwelt. Die Aktivitäten sind jedoch nicht neutral, sondern entfalten eine transformative Kraft, die sich entweder auf die Person (Kunde) richtet oder auf die Umgebung (vgl. Abb. 5.2). Die Tätigkeitstheorie unterscheidet folglich eine nach innen und eine nach außen gerichtete Tätigkeit. ${ }^{288}$ Die Transformationen lassen sich durch das Begriffspaar Aneignung - Vergegenständlichung näher beschreiben. ${ }^{289}$

- $\quad$ Aneignung (von der Umwelt zur Person)

\footnotetext{
${ }^{286}$ Leontjew (1977/ 1982), S. 52.

287 Leontjew (1977/ 1982), S. $51 \mathrm{f}$.

${ }^{288}$ vgll. Kaptelinin/ Kuutti/ Bannon (1995), S. 192; vgl. Oerter (2008), S. 99 f.; vgl. Oerter, S. 60; vgl. Leontjew, S. 47.

289 Vgl. Oerter (2008), S. 99; Oerter unterscheidet zusätzlich noch die Prozesse Objektivierung und Subjektivierung, jedoch soll dieser Unterscheidung hier nicht gefolgt werden, da Abgrenzung oft nur schwer möglich.
} 
Diese Transformation „hinterlässt Spuren oder Eindrücke beim Individuum“ “290 Die Person gewinnt ein Gefühl von Sicherheit, weil sie sich in der Umwelt orientiert und es ist eine Form der Selbstvergrößerung. ${ }^{291} \mathrm{Zu}$ den Spuren gehören z. B. Wissen und Repräsentationen. Repräsentationen sind die Art und Weise „wie oder in welchen „Kodierungen“ Erfahrungen, kommunizierte Informationen, Ergebnisse von Denken gespeichert werden“. ${ }^{292}$ Beispiele für aneignende Tätigkeiten sind Musik hören und den Umgang mit Objekten und Werkzeugen lernen. ${ }^{293}$

- Vergegenständlichung (von der Person zur Umwelt)

Diese Transformation „führt zu Ergebnissen, die längere oder kürzere Zeit fortbestehen. ${ }^{“ 294}$ Eine Person erzeugt Objekte oder verändert Objekte in der Umwelt. ${ }^{295}$ Sie ist die Voraussetzung, damit eine Person sich selbst als Akteur erfährt und ihre Wirkung auf die Umwelt erleben kann. Vergegenständlichende Tätigkeit geht mit dem Gefühl der Kontrolle über die Umwelt einher und ist ebenfalls eine Form der Selbstvergrößerung. ${ }^{296}$ Beispiele für diese Tätigkeiten sind die eigene Wohnung einrichten, Werkzeug herstellen, einen Roman schreiben oder einen Vortrag halten. $^{297}$

\subsection{Bedeutung des Anbieters bei den Kundenaktivitäten}

Das Ziel von Anbietern kann es sein, die Effizienz und Produktivität der eigenen Prozesse zu steigern, indem Kunden sich diese besser aneignen. ${ }^{298}$ Nimmt der Anbieter hingegen die Ziele des Kunden in den Blick, kann es ihm zum einen darum gehen, die Kosten des Kunden durch eine bequeme Angebotsnutzung zu reduzieren (vgl. Kap. 5.1). Bei der Bequemlichkeit handelt es sich um eine relative Größe, d. h. etwas ist bequem, wenn eine Alternative mehr Zeit und Mühe erfordert. ${ }^{299}$ Die wahrgenommene Convenience wirkt sich signifikant positiv auf die Kundenzufriedenheit aus. ${ }^{300}$ Zur erlebten Convenience kann u. a. Personalisierung beitragen. Hierrunter lassen sich auf einen Kunden angepasste Leistungen fassen, die automatisch, d. h. ohne Zutun der Person, erstellt werden. Die Leistungserstellung er-

\footnotetext{
290 Oerter (2008), S. 99.

291 Vgl. Oerter (2008), S. 99 f.

292 Oerter/ Montada (2008), S. 971.

293 Vgl. Oerter (2000), S. 61.

294 Oerter (2008), S. 99.

295 Vgl. Oerter (2000), S. 60.

296 Vgl. Oerter (2008), S. 99 f.

297 Vgl. Oerter (1999), S. 300; vgl. Oerter (2000), S. 61.

${ }^{298}$ Vgl. Frauendorf (2006), S. 81, S. 114 f.

299 Vgl. Reith (2007), S. 28.

${ }^{300}$ Vgl. Reith (2007), S. 40, S. 156.
} 
folgt durch die Datenverarbeitung eines technischen Systems auf Basis von Daten aus dem Kundenprofil und/oder mittels Daten über die Nutzungssituation (Ort, Zeit etc.). ${ }^{301}$ Personalisierte Leistungen sind aus Sicht der Tätigkeitstheorie Handlungen, die durch eine andere Person oder eine Maschine ausgeführt werden (Ebene der Operationen). Die Personalisierung bietet sich für den Kunden dann an, wenn er den Aufwand, den er bei einer Handlung investieren muss, vermeiden möchte. ${ }^{302}$ Aus diesem Grund besteht die Annahme, dass für Kunden Personalisierung in ganz unterschiedlichen Situationen bei der Angebotsnutzung hilfreich sein kann. Zum anderen kann der Anbieter versuchen, dem Kunden zu helfen produktiv(er) zu sein. ${ }^{303}$ Hierfür bietet sich u. a. Customization an. Sie beschreibt allgemein die Möglichkeit des Kunden, ein Angebot an seine Bedürfnisse und seine Vorstellungen anzupassen. ${ }^{304}$ Die Folgen der Anpassung betreffen nur die Person selbst. Niklas versteht hierunter die Adaptierbarkeit einer App, d. h. „wie flexibel ein System ist, respektive zu welchem Ausmaß das System sowie dessen Output an unterschiedliche Bedingungen und Anforderungen angepasst werden können. “305 Wolf spricht von Selektivität einer App und meint damit „das Potenzial für Rezipienten aus vorab festgelegten, standardisierten Handlungsoptionen zu wählen. ${ }^{306}$ Bei Customization handelt es sich um vergegenständlichende Aktivitäten, da Kunden die Gestalt von Objekten oder der Umgebung verändern und somit in die Welt eingreifen.

Folglich hängt das Optimierungspotenzial von Aktivitäten einerseits davon ab, ob die Kosten des Kunden reduziert werden können. Andererseits setzt das Ziel der Optimierung voraus, dass es Wege gibt, wie Kunden ihre Produktivität verbessern können oder dass Aktivitäten existieren, die neu und nützlich für Kunden sind. ${ }^{307}$ Die Rolle des Anbieters sieht in diesem Fall vor, Aktivitäten zu ermöglichen, zu erleichtern und zu unterstützen, die Kunden als wertvoll erleben. ${ }^{308}$ Hierfür stellt der Anbieter dem Kunden seine Leistungen und Potenziale als Gestaltungsfaktor bereit. $^{309}$ Die Ausstattung mit Kapazitäten und Ressourcen des Anbieters bilden den begrenzenden Rahmen, in dem ein Anbieter Entscheidungen treffen kann. ${ }^{310} \mathrm{Um}$ Optimierung realisieren zu können, muss der Anbieter zusätzlich über die nötige Gestaltungskompetenz verfügen. Damit ist die Fähigkeit gemeint, „die im Nutzungsprozess des Kunden stattfindenden Einzelprozesse zu analysieren, die hinter diesen Prozessen stehenden Bedürfnisse zu identifizieren und dem Kunden dar-

\footnotetext{
${ }^{301}$ Vgl. Rauscher/ Hess (2005), S. 7; vgl. Schedl/ Flexer/ Urbano (2013), S. 526.

${ }^{302}$ Vgl. Krippendorf (2013), S. 112.

303 Vgl. Weiber/ Hörstrup/ Mühlhaus (2011), S. 115 f.

${ }^{304}$ Vgl. Jürgens/ Stark/ Magin (2014), S. 257; Vgl. Rauscher/ Hess (2005), S. 7 f.

${ }^{305}$ Niklas (2015), S. 102.

306 Wolf (2014), S. 92.

307 Vgl. Hörstrup (2012), S. 111.

308 Vgl. Mickelsson (2014), S. 44; vgl. Heinonen et al. (2010), S. 537, S. 545.

309 Vgl. Hörstrup (2012), S. 73.

${ }^{310}$ Vgl. Heinonen/ Strandvik (2015), S. 477; vgl. Matzler/ Stahl/ Hinterhuber (2009), S. 6.
} 
aufhin angepasste Leistungen zu offerieren.“311 Dieses Marketing-Konzept wird auch als Anbieterintegration bezeichnet, weil nicht der Kunde in die Prozesse des Anbieters integriert wird, sondern der Anbieter in die Aktivitäten (Nutzungsprozesse) des Kunden. ${ }^{312}$ Die Perspektive der Customer-Dominant Logic (CDL) geht noch einen Schritt weiter und stellt die Frage in den Mittelpunkt, wie Kunden verschiedene Anbieter und Produkte in ihr Leben und in ihre verschiedenen Aktivitäten im Alltag integrieren. ${ }^{313}$ Die CDL geht zurück auf die nordische Schule aus Finnland. ${ }^{314}$ Es handelt sich um eine Perspektive, bei der Kunden im Vergleich zu anderen Aspekten wie Produkt, Kosten oder Wachstum eine herausragende Rolle spielen. Sie soll Denken und Handeln von Managern beeinflussen und sie anleiten, Märkte und Kunden zu verstehen. ${ }^{315}$

Zentral ist der Begriff der Kundenlogik, denn diese bestimmt das Denken und Handeln der Kunden. Nach Heinonen et al. handelt es sich um ein koordinierendes Konzept, das Aktivitäten des Kunden, seine Erfahrungen und Erlebnisse sowie seine Ziele integriert, aber auch seine Erwartungen und Bedürfnisse beinhaltet sowie die Art und Weise, wie Kunden ihrem Tun einen Sinn verleihen. ${ }^{316}$ Die Kundenlogik gibt darüber Aufschluss, wie Kunden ihre Aufmerksamkeit und Energie auf Angebote verteilen, welche Angebote sie auswählen und wie sie das Angebot erleben. ${ }^{317}$ Die Kundenlogik ist den Kunden nur z. T. bewusst (vgl. Kap. 5.2.2). ${ }^{318}$

Damit Anbieter ihre Kunden unterstützen können, stehen sie vor der Herausforderung die Kundenlogik und damit auch den Kontext der Angebotsnutzung besser zu verstehen. ${ }^{319}$

Im empirischen Teil der Arbeit wird der Frage nachgegangen, welchen Aktivitäten Kunden bei E-Book-Mietangeboten nachgehen und ob sie sich bei den Aktivitäten Möglichkeiten der individuellen Anpassung (Customization) und Möglichkeiten zur Personalisierung wünschen.

\subsection{Bedeutung von Aktivität für Kunden und Anbieter}

Es lässt sich feststellen, dass Personen Objekte lieber mögen, wenn sie Arbeit in diese investiert haben bzw. aktiv waren. Norton et al. bestätigen dies in ihrer Studie und bezeichnen die erlebte Wertsteigerung (höhere Zahlungsbereitschaft) durch

\footnotetext{
311 Vgl. Hörstrup (2012), S. 137.

312 Vgl. Weiber/ Hörstrup/ Mühlhaus (2011), S. 113.

${ }^{313}$ Vgl. Heinonen/ Strandvik (2015), S. 472, S. 474; vgl. Heinonen et al. (2010), S. 533.

${ }^{314}$ Vgl. Heinonen/ Strandvik (2015), S. 111; vgl. Eggert/ Fließ (2015), S. 117.

${ }^{315}$ Vgl. Heinonen/ Strandvik (2015), S. 475 f.; vgl. Heinonen/ Strandvik (2015), S. 111.

${ }^{316}$ Vgl. Heinonen/ Strandvik (2015), S. 477 f.; vgl. Mickelsson (2014), S. 16.

317 Vgl. Heinonen/ Strandvik (2015), S. 477 f.; vgl. Heinonen/ Strandvik (2015), S. 122.

318 Vgl. Heinonen/ Strandvik (2015), S. 478.

319 Vgl. Heinonen/ Strandvik/ Voima (2013), S. 115.
} 
Aktivität als IKEA-Effekt. ${ }^{320}$ Die Autoren erklären den Effekt durch die Befriedigung des menschlichen Grundbedürfnisses, sich durch eigenes Tun als Verursacher von Veränderungen in der Umwelt zu erleben, was auch als Erleben von Effectance bzw. Wirksamkeitserleben bezeichnet wird. ${ }^{321}$ Damit ist eines der grundlegenden Motive innerhalb der Makrostruktur einer Tätigkeit angesprochen (vgl. Kap. 5.2.2). Eine Bestätigung des positiven Einflusses der Aktivität auf die Bewertung von Eigenschaften eines virtuellen Angebots liefert Heinonen. Um den Aktivitätsgrad als Einflussfaktor auf das Werterleben zu untersuchen, macht sie die Kundenaktivität daran fest, wie viele unterschiedliche Aspekte eines Angebots (Online-Reisedienst) ein Kunde nutzt. ${ }^{322}$ Ihr gelingt der Nachweis, dass die aktivste Kundengruppe bei allen abgefragten Dimensionen (Funktionen, Zeit, Raum, Technik) auch das höchste Werterleben hat. ${ }^{323}$ Dieses Vorgehen bei der Messung von Aktivität wird auch von Oestreicher-Singer et al. gewählt, die empirisch bestätigen, dass sich die Kunden eines Angebots (Online-Musikcommunity) auf unterschiedlichen Stufen der Teilhabe befinden und im Zeitverlauf höhere Stufen erreichen können, wenn sie Aktivitäten nachgehen, die auf eine stärkere Teilhabe am Angebot ausgerichtet sind. ${ }^{324}$ Die Autoren stellten zudem fest, dass ein höherer Aktivitätsgrad (Häufigkeit der Aktivitäten) auf allen Stufen mit einer höheren Zahlungsbereitschaft einhergeht. Der Effekt ist bei Kunden, die sich auf höheren Stufen befinden, stärker ausgeprägt. $^{325}$ Damit lässt sich festhalten, dass eine hohe Kundenaktivität mit einem höheren Wertempfinden einhergeht, was die Zahlungsbereitschaft für die Angebotsnutzung positiv beeinflusst. In Angeboten, bei denen Kunden untereinander keinen/ wenig Kontakt haben, können Anbieter die Nutzungsintensität kaum durch soziale Beziehungen zwischen Kunden steigern. ${ }^{326}$ In einer solchen Umgebung ist es entscheidend, auf welche Weise die Kunden aktiv mit den Inhalten im Angebot umgehen können. Sundar ist der Auffassung, dass insbesondere Customization dazu geeignet ist, das Gefühl der Agency einer Person zu steigern. ${ }^{327}$ Unter der wahrgenommenen Agency versteht man das „Gefühl der eigenen Handlungsermächtigung“. ${ }^{328}$ Agency steht im engen Zusammenhang mit dem Wirksamkeitserleben. ${ }^{329}$ Dieses Erleben setzt ein, wenn der Kunde sich z. B. mithilfe von Customization selbst als Verursacher der veränderten Umgebung wahrnehmen kann bzw. sich in der Benutzungsoberfläche und/oder in den Objekten, die er erzeugt hat, selbst erkennen

\footnotetext{
${ }^{320}$ Vgl. Norton/ Mochon/ Ariely (2012), S. 455, S. 457 f.; „IKEA“ bezieht sich auf das Einrichtungsunternehmen.

${ }^{321}$ Vgl. Norton/ Mochon/ Ariely (2012), S. 454, S. 459; vgl. auch Aellig (2004), S. 22; vgl. auch Klimmt/ Hartmann (2006), S. 137; vgl. auch Klimmt/Blake (2012), S. 66, S. 74.

322 Vgl. Heinonen (2009), S. 3.

${ }^{323}$ Vgl. Heinonen (2009), S. 11.

${ }^{324}$ Vgl. Oestreicher-Singer/ Zalmanson (2013), S. 592, S. 595 f; S. 601.

${ }^{325}$ Vgl. Oestreicher-Singer/ Zalmanson (2013), S. 603.

${ }^{326}$ Vgl. Zalmanson/ Oestreicher-Singer (2015), S. 7.

327 Vgl. Sundar (2008), S. 62; Sundar et al. (2015), S. 60 f.

${ }^{328}$ Eichner (2007), S. 61.

${ }^{329}$ Vgl. Klimmt/ Hartmann (2006), S. 138.
} 
kann. ${ }^{330}$ Die Befriedigung dieses Bedürfnisses ist für Kunden jedoch nicht unbedingt klar benennbar (vgl. Kap. 5.2.2). Eine Nicht-Befriedigung würde sich daher in Aussagen wie „man kann beim Angebot nicht genug einstellen“ ausdrücken. ${ }^{331}$

\subsection{Bedeutung der Umgebung für Kundenaktivität}

Agency ist der entscheidende Vermittler zwischen den Gebrauchsmöglichkeiten eines Angebots und psychologischen Funktionen. ${ }^{332}$ Natürliche Objekte in der Welt liefern selbst Hinweise, wie sie funktionieren und was man mit ihnen tun kann. Dies geschieht durch Affordance, d. h. ein Mensch „nimmt nicht Objekte wahr, sondern deren Benutzbarkeit“. ${ }^{333}{ }^{334}$ Die Affordance ist keine Eigenschaft des Gegenstands, sondern sie bezieht sich immer auf die Beziehung zwischen einer Person und dem physischen Objekt in einer Umgebung. ${ }^{335}$ Während ein Mensch die Benutz-barkeit des gedruckten Buchs direkt wahrnimmt, z. B. seine Les-barkeit, sind E-Books keine Objekte. Nur Geräte/Bildschirme haben Affordance, die jedoch nicht zum Lesen anregt. ${ }^{336}$ Innerhalb der Benutzungsoberfläche von Software kann man daher nur unter bestimmten Voraussetzungen von Affordance sprechen. Die Voraussetzungen sind erfüllt, wenn der Computer die Geste einer Person z. B. „über den Bildschirm wischen“ als Befehl auffasst (die Bewegung wurde also bei Programmierung berücksichtigt) und wenn die Person sich bewusst ist, dass sie auf diese Weise eine gewünschte Reaktion hervorrufen kann. ${ }^{337}$ In diesem Fall handelt es sich um eine wahrgenommene Affordance, weil eine Person sich entweder daran erinnert, die Funktion bereits genutzt zu haben, oder sie wird durch einen wahrnehmbaren Hinweisreiz in der Umgebung nahegelegt. ${ }^{338}$ Notwendig ist immer ein sensorischer Input, mit dem eine Handlung verbunden wird, die auf ein bestimmtes Ziel ausgerichtet ist ${ }^{339}$ z. B. ein Schiebebalken zum Ziehen. ${ }^{340}$ Da Handlungen bei Software jedoch durch diese vermittelt sind, weist Affordance aus instrumenteller Sicht immer zwei Facetten auf: die physische, direkte Interaktion mit der Benutzungsschnittstelle (handling affordance) und der indirekte Effekt, der beim Objekt des Interesses (z. B. Buch) erzeugt wird (effecter affordance). ${ }^{341}$ Wenn der Punkt auf

\footnotetext{
${ }^{330}$ Vgl. Sundar et al. (2015), S. 61; vgl. Sundar (2008), S. 68; vgl. Trepte/ Reinecke (2010), S. 222.

${ }^{331}$ Vgl. Klimmt/ Hartmann (2006), S. 139 f.

${ }^{332}$ Vgl. Sundar (2008), S. 68.

333 Vgl. Krippendorf (2013), S. 149.

${ }^{334}$ Der Begriff Affordance geht zurück auf James J. Gibson und meint die „Ermöglichung von Handlungen“" (Krippendorff (2013), S. 148).

${ }^{335}$ Vgl. Krippendorf (2013), S. 150 f.; vgl. Norman (2016), S. 10.

${ }^{336}$ Vgl. Norman (1999); vgl. Nielsen/ Badiu (2013), S. 165.

337 Vgl. Nielsen/ Badiu (2013), S. 165; vgl. Norman (1999).

${ }^{338}$ Vgl. Nielsen/ Badiu (2013), S. 165.

${ }^{339}$ Vgl. Vermeulen et al. (2013), S. 8.

${ }^{340}$ Vgl. Kaptelinin (2014).

${ }^{341}$ Vgl. Kaptelinin/ Nardi (2012), S. 6.
} 
Tag und Person alle drei Jahre einen Höchstbetrag von 10000 Kronen in Devisen umzutauschen; sie hätte der DDR die Bereitstellung von 4,7 Milliarden

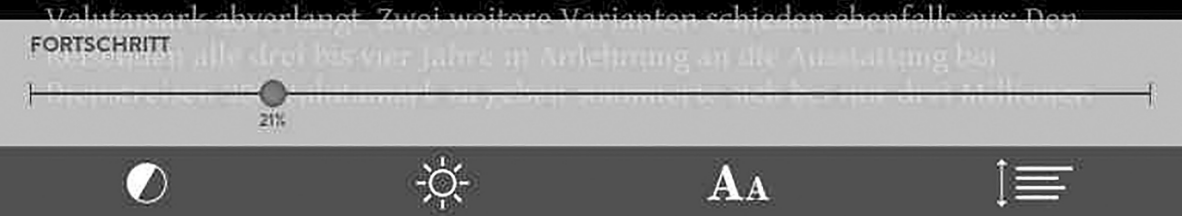

Abb. 5.3 Position und Navigation im Buch unterstützt durch Software (Quelle: Screenshot Readfy, 16.11.2014)

dem Fortschrittsbalken durch ziehen bewegt wird, entspricht dies dem Seitenwechsel im Buch (vgl. Abb. 5.3).

Zugleich signalisiert der Punkt, wie viel bereits gelesen wurde. Beim gedruckten Buch wird das Gefühl für den Lesefortschritt durch das Objekt selbst signalisiert. ${ }^{342}$ Die wahrgenommene Affordance besteht also aus einer Vorstellung über die nötigen Bewegungen und den Effekt, den diese auslösen, d. h. eine Person muss einen Zusammenhang zwischen ihrem Ziel und dem Fortschrittsbalken als geeignetem Instrument herstellen. Auch Sundar et al. konzeptualisieren Funktionen eines Angebots als Affordance. ${ }^{343}$ Diese können auf die Psyche einer Person auf zweifache Weise einen Effekt haben. Entweder führen sie dazu, dass ein Kunde eine Handlung ausführt und/oder der Kunde nutzt sie - wie oben erwähnt - als Hinweisreiz. ${ }^{344}$ Hinweisreize lassen sich als Signale auffassen. Signale sind Bestandteil von Zeichenprozessen und können in drei Typen unterteilt werden: Anzeichen, ikonische Zeichen und Symbole wie Schriftzeichen. ${ }^{345}$ Ikonische Zeichen sind entweder ein Abbild dessen, was sie repräsentieren, oder sie sind z. B. Piktogramme, die ähnlich wie Symbole auf einer Konvention beruhen und deren Bedeutung erst gelernt werden muss. ${ }^{346}$ Beispielsweise soll das Piktogramm „Lupe“ eine Suchfunktion signalisieren. Symbole, ikonische Zeichen und andere Hinweisreize zeigen die Präsenz von Funktionen an, während automatisch generierte Statistiken Kunden mit Informationen versorgen. ${ }^{347}$ Sie können als Anzeichen interpretiert werden, wenn hinter ihnen keine direkte Absicht zur Kommunikation steht, den Zahlen aber trotzdem Bedeutung zugeschrieben wird. ${ }^{348}$ Zum Beispiel signalisiert die Zahl, wie häufig ein Buch gelesen wurde, seine Beliebtheit. ${ }^{349}$

\footnotetext{
${ }^{342}$ Vgl. Norman (2016), S. 15.

${ }^{343}$ Vgl. Sundar et al. (2015), S. 50; vgl. auch Kaptelinin/ Nardi (2006), S. 63, vgl. auch Norman (2016), S. 11.

${ }^{344}$ Vgl. Sundar et al. (2015), S. 51, S. 70.

${ }^{345}$ Vgl. Beck (2013), S. 24.

${ }^{346}$ Vgl. Beck (2013), S. 25.

347 Vgl. Sundar et al. (2015), S. 51, S. 70.

348 Vgl. Beck (2013), S. 24 f.

${ }^{349}$ Vgl. Sundar et al. (2015), S. 51, S. 70; vgl. Jungnickel/ Schweiger (2014), S. 25.
} 



\section{Buchbezogene Kundenaktivitäten}

\subsection{Systematisierung von buchbezogenen Kundenaktivitäten}

Im Folgenden soll untersucht werden, welchen unterschiedlichen Aktivitäten speziell Buchleser nachgehen. Die Aktivitäten sind miteinander verbunden, weil sie das Interesse am Bücherlesen als Thema teilen (vgl. Kap. 3.1) und vom Kunden wiederkehrend ausgeübt werden, um Wert für sich zu schaffen. ${ }^{350}$ Durch ihre Verbundenheit bilden die Aktivitäten ein Set. Mickelsson bestätigt ein solches Set empirisch bei einer Befragung von Personen mit Interesse an Wein (z. B. an Weinkursen teilnehmen, Weinmagazine lesen, Weinberge im Ausland besuchen, Wein im Geschäft kaufen etc.). ${ }^{351}$ Die Aktivitäten unterstützen alle das Ziel des Kunden, seine Interessen zu verwirklichen. Hieran zeigt sich, dass Aktivitäten nicht in einer bestimmten zeitlichen Reihenfolge ausgeübt werden müssen, sondern eine Person geht diesen situativ nach, wann immer es erforderlich wird, sie den Wunsch dazu verspürt oder es zeitlich gerade passt. ${ }^{352}$ Andererseits kann Kohler nachweisen, dass Kunden ihre Aktivitäten, die im Zusammenhang mit einer übergeordneten Aufgabe stehen, in eine zeitliche Reihenfolge bringen. Kunden haben für Anlässe wie den Bucheinkauf im Laden ein Skript entwickelt (vgl. Abb. 6.1), d. h. die Aufgabe ist als ein linearer zeitlich festgelegter Ablauf von Aktivitäten im Gedächtnis repräsentiert. ${ }^{353}$ Das Skript beinhaltet keine konkreten Erinnerungen, sondern formt „eine standardisierte und generalisierte Episode, die aus vielen ähnlichen Erlebnissen heraus gebildet wird. ${ }^{\text {(354 }}$

Im Alltag sind zudem Nutzungs- bzw. Verwendungsregeln im Sinne von Erwartungen wirksam, die das Handeln von Menschen strukturieren und den wahrgenommenen Handlungsspielraum einschränken bzw. bestimmte Handlungen nahelegen. ${ }^{355}$ Die Regeln können expliziten Charakter haben durch Gesetze und rechtliche Vorschriften ${ }^{356}$ wie BGB, AGBs oder Benutzungsordnungen in Bibliotheken. Diese führen einfach ausgedrückt dazu, dass Bücher bezahlt und zurückgegeben werden, Aktivitäten also Bestandteil des Skripts werden. Andererseits sind

\footnotetext{
${ }^{350}$ Vgl. Mickelsson (2014), S. 23, S. 27, S. 111.

351 Vgl. Mickelsson (2014), S. 142.

352 Vgl. Mickelsson (2014), S. 111.

${ }^{353}$ Vgl. Kohler (2007), S. 23 ff., S. 65; Ältere Kinder üben mit jüngeren Kindern bereits spielerisch das Einkaufsskript ein (Vgl. Oerter (1999), S. 168 ff.).

${ }^{354}$ Kohler (2007), S. 26.

355 Vgl. Schmidt (2011), S. 51.

356 Vgl. Schmidt (2011), S. 53.
} 

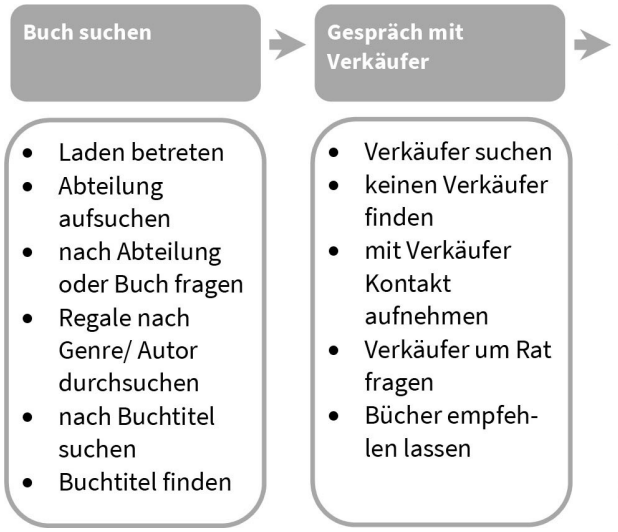

Buch auswählen und zur Kasse gehen

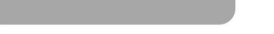

- Buch aus dem Regal nehmen

- Umschlag lesen

- im Buch blättern

- auf den Preis schauen

- sich für Buch entscheiden

- zur Kasse gehen

- Buch an Kassierer übergeben
Buch bezahlen

$\begin{array}{ll}\text { - } & \text { bezahlen } \\ \text { Buch vom } \\ \text { Kassierer zurück- } \\ \text { erhalten } \\ \text { - Kassenbon } \\ \text { erhalten } \\ \text { sich bedanken } \\ \text { und verab- } \\ \text { schieden } \\ \text { Geschäft } \\ \text { verlassen }\end{array}$

Abb. 6.1 Aktivitäten eines Skripts für den Bucheinkauf im Laden (Quelle: Eigene Erstellung nach Kohler (2007), S. 63, nur Nennungen Aktivität > 15, Ausnahme: Gespräch mit Verkäufer alle Nennungen Aktivität $<16$ )

implizite Regeln wirksam ${ }^{357}$ wie „sich bedanken und verabschieden“ (vgl. Abb. 6.1). In diesem Zusammenhang ist die Frage relevant, wie Kundenaktivitäten noch miteinander verbunden sein können. Mickelsson verweist, abgesehen von kognitiven Verbindungen z. B. durch ein Skript, auf funktionale Verbindungen, d. h. eine Tätigkeit hat instrumentellen Charakter und wird ausgeübt, um eine andere Tätigkeit zu ermöglichen. ${ }^{358}$ Beispielsweise werden Bücher gekauft oder ausgeliehen, um diese lesen zu können. Die instrumentelle Tätigkeit selbst wird als weniger befriedigend erlebt. ${ }^{359}$ Daneben kann das gewonnene Wissen bei einer Tätigkeit (z. B. einen interessantes Buch beim Stöbern entdeckt) als Ressource in eine andere Tätigkeit integriert werden $^{360}$ z. B. bei der Auswahl von Büchern oder bei Gesprächen mit anderen über Bücher.

\subsection{Entwicklung eines Sets von buchbezogenen Kundenaktivitäten}

Im Folgenden soll ein Set von buchbezogenen Aktivitäten entwickelt werden. Dabei geht es nicht um Vollständigkeit, sondern darum, solche Aktivitäten zu identifizieren, die einen direkten Bezug zum Bücherlesen haben und für die ein Anbieter

\footnotetext{
357 Vgl. ebd.

${ }^{358}$ Vgl. Mickelsson (2014), S. 46, S. 143.

359 Vgl. ebd.

360 Vgl. ebd.
} 
Leistungen/Potenziale zur Individualisierung bereitstellen kann (vgl. Kap. 5.3). Brown et al. decken in ihrer qualitativen Studie einen Lebenszyklus der Musiknutzung auf: auf Musik aufmerksam werden, Musik erwerben, Musik hören, Musik auswählen, Musik kopieren und zusammenstellen, Musik organisieren und Musik sammeln. ${ }^{361}$ Der Lebenszyklus lässt sich größtenteils auf Bücher übertragen. Sie vermittelt einem Anbieter einen Eindruck davon, bei welchen Aktivitäten er seine Kunden unterstützen kann:

- Bücher erwerben

- Bücher lesen

- Bücher auswählen

- Bücher aufbewahren und sammeln

- Bücher und Informationen organisieren

Die Rolle des Buches als Sammelobjekt ist in dieser Arbeit nicht relevant (vgl. Kap. 3.1). Die Tätigkeit des Sammelns kann aber auch dem Ziel dienen, Bücher zu einem späteren Zeitpunkt zu lesen. ${ }^{362}$ Um diese Form des Sammelns geht es bei der Organisation von Büchern, aber auch von Informationen insgesamt. Schmidt fasst solche Aktivitäten unter dem Begriff des Informationsmanagements zusammen, das er „im Sinne von „Handhaben“ oder ,Bewerkstelligen “ ${ }^{363}$ versteht. Die Aktivitäten beim Erwerb wurden anhand des Einkaufsskripts veranschaulicht (vgl. Abb. 6.1). Es zeigt sich, dass diese sich v. a. dem Such- und/oder Auswahlprozess zuordnen lassen. Auf nicht buchtypisch ausgeprägte Aktivitäten wie „Bezahlen“ wird nicht weiter eingegangen.

\subsection{Bücher lesen}

Die Funktionen in Lesesoftware lassen sich in der Realität durch Autopsie von Angeboten finden und müssen nicht Bestandteil jeder Software sein. ${ }^{364}$ Bei einer Befragung im Jahr 2002 wurde ermittelt, wie wichtig bestimmte Funktionen für die Nutzung von E-Books sind. ${ }^{365}$ Zur Stichprobe gehörten Personen, die sich aus beruflichen Gründen mit E-Books beschäftigen und zugleich E-Book-Leser repräsentieren. ${ }^{366}$ U. a. werden Funktionen als wichtig bewertet, die erst beim softwarebasierten Lesen möglich sind, weil ein gedrucktes Buch keine eigene Verarbeitungskapazität aufweist ${ }^{367}:$ Zu den wichtigsten zählen „Buch öffnet sich auf

\footnotetext{
${ }^{361}$ Brown/ Geelhoed/ Sellen (2001), S. 3.

${ }^{362}$ Vgl. Rudolph (2014), S. 212.

${ }^{363}$ Schmidt (2011), S. 74.

${ }^{364}$ Vgl. Engesser (2013), S. 109.

365 Vgl. Henke (2002), S. 6.

366 Vgl. Henke (2002), S. 8.

367 Vgl. Mowshowitz (1992), S. 236.
} 
zuletzt angesehener Seite“ (4,49 auf 5er-Skala/Rang 1 von 48) und „Darstellung ändern“ (4,2/Rang 9). ${ }^{368}$ Die beiden Funktionen verweisen zugleich auf die nötige Trennung zwischen kunden- und systeminitiierter Individualisierung, die durch die Begriffe Customization und Personalisierung erfolgt (vgl. Kap. 5.3). ${ }^{369}$ Die Funktion „Buch öffnet sich auf zuletzt angesehener Seite“ ist eine Form der Personalisierung.

Merkmal einer Dimension von Optimierungspotenzial: Personalisierung beim Lesen der Bücher

Die Möglichkeit der Darstellungsänderung ist hingegen eine Form von Customization.

Merkmal einer Dimension von Optimierungspotenzial: Customization beim Lesen der Bücher

Ob für den Kunden Customization bei der Typografie möglich ist, hängt davon ab, ob Anbieter bzw. Hersteller eine kontrollierte Typografie vorsehen und in welcher Struktur die Buchdaten vorliegen. ${ }^{370}$ Bei einer festen Typografie werden Eigenschaftsausprägungen wie beim gedruckten Buch gestaltet und fixiert z. B. ist die Darstellung eines E-Books im PDF-Format auf jedem Gerät einheitlich. ${ }^{371} \mathrm{Zu}$ den anpassbaren typografischen Gestaltungsmitteln gehören Schriftart und Schriftgröße, Buchstabenabstand (Laufweite, Unterschneiden), Wortabstand, Trennungen am Zeilenende und Zeilenabstand. ${ }^{372}$ Ist Customization vorgesehen, entscheidet der Leser selbst, ob er diese nutzt oder die Voreinstellungen beibehält. ${ }^{373}$ Weitere Handlungen, die auch im Umgang mit gedruckten Büchern eine Rolle spielen, sind „automatische Textsuche“ (4,44/Rang 3), „Lesezeichen setzen“ (4,43/Rang 4), „etwas hervorheben/markieren“ (3,86/Rang 13), „Wörterbuch benutzen“ (3,73/Rang 16), „Anmerkungen/Notizen erstellen“ (3,6/Rang 19) und „Thesaurus benutzen“ (2,89/ Rang 35). ${ }^{374}$ Tatsächlich hin und wieder von E-Book-Lesern genutzt werden „Lesezeichen setzen“ (69\%), „automatische Textsuche“ (38\%), „Notizen erstellen“ (26\%), „Markierungen vornehmen“ (20\%) und „Übersetzungen/Erklärungen nachschlagen“ $(17 \%){ }^{375}$ Pette bezeichnet solche Handlungen als Lesestrategien: „all jene kognitiven, emotionalen und sozialen Handlungen (.), die der Leser im Zusammenhang mit der Rezeption vollzieht.““376 Es sind „Handlungen, die der Leser vollzieht, um den Leseprozeß derart zu gestalten, daß er seinen Erwartungen, Vorein-

\footnotetext{
${ }^{368}$ Vgl. Henke (2002), S. 10.

369 Vgl. Sundar/ Marathe (2010), S. 300.

${ }^{370}$ Vgl. Kuhn/ Hagenhoff (2015), S. 320 f.

371 Vgl. Kuhn/ Hagenhoff (2015), S. 320 f.

${ }^{372}$ Vgl. Spitzmüller (2016), S. 216.

${ }^{373}$ Vgl. Hagenhoff/ Kuhn (2014), S. 223 f.

374 Vgl. Henke (2002), S. 10 f.

375 Vgl. Bitkom (2014), S. 7.

${ }^{376}$ Pette (2001), S. 53.
} 
stellungen und Bedürfnissen möglichst gerecht wird““. ${ }^{377}$ Sie helfen dem Leser u. a. bei Problemen, die sich im Zuge der Bedeutungskonstruktion ergeben, z. B. bei unbekannten Wörtern, und dienen dann dem Ziel der Verstehenssicherung. ${ }^{378}$

\subsection{Bücher auswählen}

Bei der Angebotsgestaltung kann es dem Anbieter darum gehen, den Kunden zu stimulieren und seine gewohnten Entscheidungsheuristiken zu unterstützen. ${ }^{379}$ Seine Gestaltungsentscheidungen korrespondieren idealerweise damit, wie Kunden Bücher suchen und ermöglichen es ihnen, Bücher nach gewohnten Kriterien auszuwählen. Der Auswahlprozess wird durch Navigationswerkzeuge und Informationen bestimmt, die der Anbieter dem Kunden zur Verfügung stellt. Suchaufgaben von Lesern haben typischerweise folgende Ausgangspunkte ${ }^{380}$ :

Mit Suchrichtung

- $\quad$ Autor/Titel finden (konkrete Vorgabe)

- Titel zu Thema finden (Vorgabe: bestimmtes Thema)

- Analogie-Suche (Vorgabe: Titel finden, die einem bereits gelesenen Titel ähneln)

Ohne Suchrichtung

- Stöbern (Vorgabe: Titel finden, die man lesen möchte)

Suchprozesse setzten sich aus Mikrostrategien zusammen, die Kunden anwenden um persönliche Suchaufgaben zu bewältigen: Suchanfrage stellen, Linking (Verbinden), Scanning (Überfliegen), Evaluation. ${ }^{381}$ Die Strategien unterliegen keiner festen zeitlichen Reihenfolge, sondern Auswahl und Kombination erfolgt in einer konkreten Situation je nach gewähltem Einstiegspunkt der Suche. Zudem müssen in einer Situation nicht alle Strategien angewendet werden. Es existieren verschiedene Navigationswerkzeuge, die den Kunden in die Lage versetzen, seine Suchaufgaben erfolgreich zu erledigen. Im empirischen Teil der Arbeit wird geprüft, ob Kunden bei der Suche von Büchern Möglichkeiten der Personalisierung oder Customization erwähnen.

Merkmale einer Dimension von Optmimierungspotenzial: Customization bei der Suche nach Büchern, Personalisierung bei der Suche nach Büchern

\footnotetext{
377 Vgl. Charlton/ Pette (1999), S. 106.

378 Vgl. Charlton/ Pette (1999, S. 109, S. 111.

379 Vgl. Müller-Hagedorn/ Toporowski/ Zielke (2012), S. $712 \mathrm{f}$.

380 Vgl. Mikkonen/ Vakkari (2016), S. 62; vgl. Thudt/ Hinrichs/ Carpendale (2015), S. 5.

${ }^{381}$ Vgl. Thudt/ Hinrichs/ Carpendale (2015), S. 1.
} 
Die Mikrostrategien und Navigationswerkzeuge werden nachfolgend veranschaulicht.

\subsubsection{Evaluation von Büchern}

Bei der Evaluation beurteilt eine Person den Titel im Hinblick auf ihr Suchziel. Das Ergebnis ist eine Entscheidung dafür oder dagegen. ${ }^{382}$ Zur Entscheidungsfindung werden dem Titel Attribute/Eigenschaften zugeschrieben. ${ }^{383}$ Es lassen sich interne und externe Quellen der Informationen unterscheiden, die während der Evaluation eine Rolle spielen können. Interne Informationen sind das Wissen, das im Langzeitgedächtnis einer Person gespeichert ist und in einer Situation willentlich ins Bewusstsein gerufen wird, während externe Informationen situativ angeregt durch Stimuli in der Umgebung aufgenommen werden. ${ }^{384}$ Bei der Evaluation lassen sich die direkte und die indirekte Evaluation unterscheiden. ${ }^{385}$ Direkte Evaluation meint das Lesen im Buch und die indirekte Evaluation die Prüfung von externen Informationen wie Autor, Cover, Titel etc. sowie den Rückgriff auf Vorwissen. ${ }^{386}$ Der hohe kognitive Aufwand bei der direkten Evaluation fördert das Verhalten auf Basis des verfügbaren Wissens zu entscheiden, um Ressourcen zu sparen. ${ }^{387}$ Bei der Entscheidung dafür (kaufe ich bzw. lese ich) werden am häufigsten die Attribute Autor, Genre, Thema und Schreibstil berücksichtigt. ${ }^{388}$ Wenn es darum geht, die Auswahl auf potenziell geeignete Titel einzugrenzen (kaufe bzw. lese ich vielleicht/kaufe bzw. lese ich nicht), sind die am häufigsten genannten Attribute ebenfalls Autor, Genre und Thema. ${ }^{389}$ In dieser Phase (Eliminationsphase) werden jedoch im Durchschnitt mehr Attribute herangezogen als bei der finalen Entscheidung. ${ }^{390}$ Folgende Kriterien werden mit hoher Wahrscheinlichkeit öfter (d.h. hauptsächlich) beim Eingrenzen genutzt: Inhalt ist unklar (13\%), nicht Muttersprache (8\%), Auszeichnung (6\%), Titelname (5\%), Klassiker (4,2\%), Frauen- oder Minderheiten (4,1\%), Film/ Fernsehserie gesehen $(3,7 \%)$, positives Image des Verlages oder der Verlagsreihe $(3,3 \%) .^{391}$

\footnotetext{
${ }^{382}$ Vgl. Thudt/ Hinrichs/ Carpendale (2015), S. 4.

383 Vgl. Schweiger (2007), S. 174.

384 Vgl. Kroeber-Riel/ Gröppel-Klein (2013), S. 339; vgl. Ross (1999), S. 788 f.

385 Vgl. Schweiger (2007), S. 187.

${ }^{386}$ Vgl. Thudt/ Hinrichs/ Carpendale (2015), S. 4.

387 Vgl. Schweiger (2007), S. 189; vgl. Leemans/ Stokmans (1991), S. 496.

${ }^{388}$ Vgl. Leemans/ Stokmans (1991), S. 497.

389 Vgl. ebd.

${ }^{390}$ Vgl. Leemans/ Stokmans (1991), S. 496.

391 Vgl. Leemans/ Stokmans (1991), S. 499.
} 


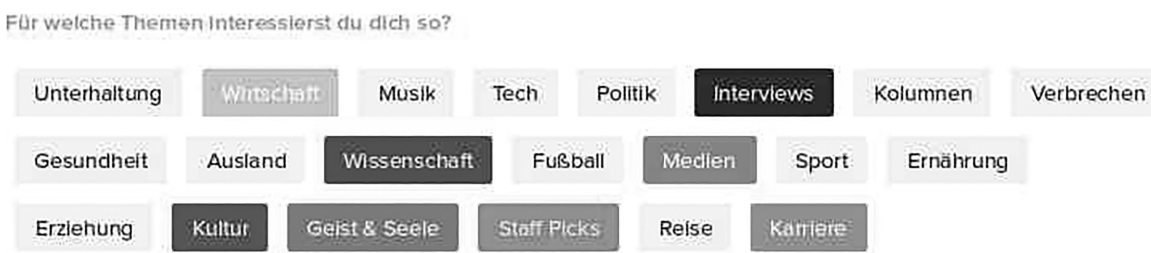

Abb. 6.2 Customization durch Festlegung von Themenpräferenzen (Quelle: Screenshot Blendle, 25. 08. 2016, https://blendle.com)

\subsubsection{Scannen von Büchern}

Beim Scanning (Überfliegen) werden visuelle Aspekte ohne erkennbare Interaktion mit einem Buch wahrgenommen. Die Strategie Scanning ist wichtig, um einen Buchtitel in einer Sammlung zu finden, sich einen Überblick über eine Sammlung zu verschaffen oder allgemein um zu Stöbern. ${ }^{392}$ Ordnungsprinzipien der Anbieter helfen dem Kunden, den Bestand auf relevante Produkte einzugrenzen ${ }^{393}$ Eine gleichzeitige Präsentation aller Buchtitel erfolgt z. B. im Landen ${ }^{394}$, d. h. die Anzahl der Optionen ist sichtbar beschränkt. In dieser Situation steigt die Wahrscheinlichkeit attributorientierter Heuristiken z. B. wird ein Attribut zur Eingrenzung gewählt und um die Entscheidung weiter zu vereinfachen wird ein weiteres Attribut herangezogen.$^{395}$ Um für das Scannen eine handhabbare Menge an Optionen zu erhalten, blenden Personen bei der Suche also nicht relevante Optionen aus ${ }^{396}$, was auch durch eine attributweise Anordnung der Optionen nahegelegt werden kann. ${ }^{397}$ Um Kunden diese Mikrostrategie zu erleichtern, können Anbieter Customization ermöglichen z. B. indem ein Kunde seine generelle Präferenz oder Ablehnung für bestimmte Bucharten oder Themen ausdrücken kann (vgl. Abb. 6.2).

Die favorisierten Bücher erscheinen dann z. B. in einer Rubrik „Persönliche Empfehlungen“. Abgelehnte Themen/Bucharten werden z. B. für Empfehlungen nicht mehr berücksichtigt. Hierfür müssen Bücher inhaltlich nach einer sachbegrifflichen Ordnung erschlossen werden, was eine typische Aufgabe ist, die von Bibliotheken wahrgenommen wird. ${ }^{398}$ Das Scannen von Sammlungen wird erschwert, wenn nur der Buchrücken sichtbar ist z. B. bei der Regalpräsentation in einer Bibliothek oder im Laden), weil Kunden dann zur Aufnahme von Informationen die Bücher aus dem Regal ziehen müssen, was das Scannen aufwändiger

\footnotetext{
${ }^{392}$ Vgl. Thudt/ Hinrichs/ Carpendale (2015), S. 4.

${ }^{393}$ Vgl. Kalyanaraman/ Wojdynski (2015), S. 434.

${ }^{394}$ Vgl. Schweiger (2007), S. 180; vgl. Wirth/ Schweiger (1999), S. 60.

395 Vgl. Schweiger (2007), S. 176.

396 Vgl. Laplante (2010), S. 603; vgl. Thudt/ Hinrichs/ Carpendale (2015), S. 8.

397 Vgl. Wirth/ Schweiger (1999), S. 56 f.

${ }^{398}$ Haller/ Fabian (2004), S. 222.
} 
macht. ${ }^{399}$ Das Explorieren der Trefferliste einer Suchanfrage in einem Online-Katalog wird erschwert, wenn nur die Informationen Autor und Titel angezeigt werden, da die mögliche Passung nicht abgeschätzt werden kann, wenn der Autor nicht bekannt ist. Daher ist es sinnvoll, die Trefferliste z. B. mit Buchcover, einem Ausschnitt aus der Kurzbeschreibung und Leserbewertungen anzureichern. ${ }^{400}$ Die nachgewiesene kürzere Beschäftigung mit einer angereicherten Trefferliste im Suchprozess könnte darauf hindeuten, dass Kunden schneller potenziell geeignete Titel identifizieren. ${ }^{401}$ Allgemein lässt sich bilanzieren, dass es für Kunden hilfreich sein kann, die Strategien Scanning und Evaluation (wahlweise) parallel anwenden zu können. ${ }^{402}$ Darüber hinaus ist es bei einer listenförmigen Präsentation einer Menge von Optionen generell der Fall, dass diese, je nach Umfang, zu Ermüdungserscheinungen beim Lesen und Navigieren führen kann. ${ }^{403}$

\subsubsection{Verbindungen zwischen Büchern wahrnehmen}

Auch Verbindungen eignen sich als Suchstrategie ohne zur Trefferliste einer Suchanfrage zurückkehren zu müssen. ${ }^{404}$ Es lassen sich Item-to-Item- und Item-to-SetVerbindung unterscheiden ${ }^{405}$ :
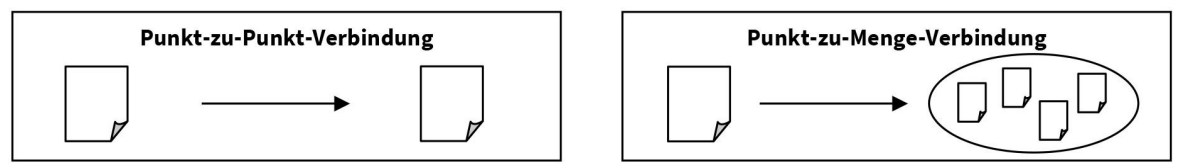

Abb. 6.3 Visualisierung der Suchstrategie „Verbindungen folgen“ (Quelle: Eigene Erstellung in Anlehnung an Thudt/ Hinrichs/ Carpendale (2015), S. 4)

Navigationswerkzeuge, die es dem Kunden ermöglichen, Verbindungen zu folgen, werden in der Arbeit als explorative Werkzeuge bezeichnet, weil Kunden keine konkreten Suchvorgaben haben müssen, um in einer Sammlung von Büchern navigieren bzw. sich bewegen zu können.

In virtuellen Angeboten sind Verbindungen softwarebasierte Relationen in Form von Hyperlinks, die sich zur Navigation eignen. ${ }^{406}$ Bei gedruckten Büchern wird die Suchstrategie Titel-zu-Titel-Verbindung z. B. beim Nachverfolgen eines Hinweises

\footnotetext{
399 Vgl. Hinze et al. (2012), S. 6.

400 Vgl. Mikkonen/ Vakkari (2016), S. 66.

401 Vgl. ebd.

${ }^{402}$ Vgl. Thudt/ Hinrichs/ Carpendale (2015), S. 8.

${ }^{403}$ Vgl. Nielsen/ Badiu (2013), S. 152.

${ }^{404}$ Vgl. Mikkonen/ Vakkari (2016), S. 67.

405 Vgl. Thudt/ Hinrichs/ Carpendale (2015), S. 4.

${ }^{406}$ Vgl. Schmidt (2011), S. 56.
} 


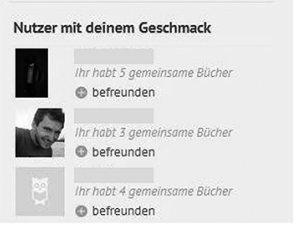

\begin{abstract}
Abb. 6.4 Personalisierung durch algorithmisch erzeugte Empfehlungen von Kundenprofilen (Quelle: Screenshot Readgeek, 11.08. 2016, http://www.readgeek.com/)
\end{abstract}

aus einer Fußnote in einem Buch zum zitierten Titel angewandt oder wenn Bücher physisch nebeneinander platziert werden. Daneben kann eine Person ausgehend von einem Titel eine andere Person um eine Empfehlung für einen ähnlichen Titel bitten. ${ }^{407}$ Auch technische Empfehlungssysteme können eine Titel-zu-Titel-Verbindung generieren. ${ }^{408}$ Hier wird die Handlung, jemanden um Rat fragen, von einem Algorithmus durchgeführt. Es handelt sich um eine Form der Personalisierung, weil Kunden keine Bücher suchen müssen. ${ }^{409}$ Stattdessen präsentiert das System basierend auf dem Kundenverhalten Buchvorschläge aus einer gegebenen Menge an Büchern. ${ }^{410}$ Neben Büchern kann ein Algorithmus auch Personen empfehlen ${ }^{411}$, sodass Kunden diese Verbindungen zum Navigieren nutzen können. Die Übereinstimmung lässt sich z. B. davon ableiten, wie viele gemeinsame Bücher sich in den Leselisten befinden (vgl. Abb. 6.4).

Verbindungen zwischen Titeln sind besonders beim Stöbern und bei der Analogie-Suche hilfreich. Dies kann über eine Gruppierung von Büchern nach einem gemeinsamen Merkmal erfolgen z. B. derselbe Autor, dasselbe Genre, das gleiche Thema (Titel-zu-Menge-Verbindung). Jedoch ist dieses Werkzeug weniger erfolgsversprechend, wenn Titel gesucht werden, die ein gefühlsmäßig ähnliches Leseerlebnis versprechen. In diesem Fall sind Leseerfahrungen anderer Menschen hilfreicher. ${ }^{412}$ Die Suche unterliegt dann keinen technischen Beschränkungen, sondern der Sucherfolg hängt davon ab, ob das Lesebedürfnis sprachlich ausgedrückt werden kann. ${ }^{413}$ Ein Werkzeug, das in solchen Situationen infrage kommt, sind die Leselisten anderer Kunden. Es eignet sich sowohl zum Stöbern als auch generell bei einer Analogie-Suche. ${ }^{414}$ Ebenso sind Tags in beiden Fällen nützlich, weil sie einen alternativen Navigationspfad bieten und themenbezogenes Stöbern erleichtern. ${ }^{415}$ Ausgehend von einem Schlagwort ist eine Stichwortsuche möglich. ${ }^{416}$ Von einem Buchtitel aus können Kunden auf alle Buchtitel zugreifen, die ebenfalls getaggt

\footnotetext{
${ }^{407}$ Vgl. Ross (1999), S. 789; vgl. Ooi/ Liew (2011), S. 758 f.

${ }^{408}$ Vgl. Thudt/ Hinrichs/ Carpendale (2015), S. 4.

409 Vgl. Jürgens/ Stark/ Magin (2015), S. 251

${ }^{410}$ Vgl. Reimer (2013), S. 238; vgl. Núñez-Valdéz (2012), S. 1446.

411 Vgl. Gärtner (2012), S. 29.

${ }^{412}$ Vgl. Thudt/ Hinrichs/ Carpendale (2015), S. 5.

${ }^{413}$ Vgl. Koolen et al. (2015), S. 2, S. 4.

${ }^{414}$ Vgl. für Musik Weigl/ Guastavino (2011), S. 338; vgl. auch Laplante (2010), S. 603.

${ }^{415}$ Vgl. Drenner/ Sen/ Terveen (2008), S. 189.

${ }^{416}$ Vgl. Peters (2013), S. 233.
} 


\section{Beliektetans \\ 20082009201020112012 Abenteuer abgebrochen Action Afrika Austratien Behaltì berlin biografie Biographie chira comic Deutschland Drama ebook england englisch Erotik fantasy Frankreich gellehen geschichte historisch herror Humor Horbuch iNoren Irland italien japan fugand Jugendbuch kinder kinderbuch klassiker komadle Krieg krimi kunat kurzgeschichten Liebe Magie mittelaiter Musik mystery marchen philosophie politik saligion Roman russiand Sachbuch Satire Schottiand schveden spanien SUb Thriller USA vampire vertauscht WB weihnachten Zeitreise}

Abb. 6.5 Tagcloud als Navigationswerkzeug (Quelle: Screenshot Reliwa, 11.08. 2016, http:// www.reliwa.de/)

wurden sowie auf die Nutzerprofile, welche die Titel in ihrer öffentlichen Leseliste gespeichert haben. ${ }^{417}$ Die Summe aller Schlagworte kann als Tagcloud dargestellt werden (vgl. Abb. 6.5).

\subsubsection{Suchanfragen für Bücher stellen}

Explorative Navigationswerkzeuge erfahren nachweislich weniger Aufmerksamkeit bei einer klar umrissenen Suchaufgabe (Autor oder Thema steht fest). ${ }^{418} \mathrm{Um}$ in einem virtuellen Angebot Suchanfragen stellen zu können ist eine Suchmaschine nötig. In einer Bibliothek oder im Laden kann auch Personal kontaktiert werden. Der Großteil der Personalanfragen im Laden sind Orientierungsfragen. ${ }^{419}$ In Bibliotheken unterstützt die im Vorfeld identifizierte Nummer eines Titels die Suche. ${ }^{420}$ Buchanan/McKay untersuchen Personalanfragen in Buchhandlungen dahingehend, welche Informationen Besucher den Mitarbeitern konkret bereitstellen $^{421}$. Dies sind zum einen bibliografische Daten, also Titel oder Teile davon ( $n=206)$ und der Autor als Startpunkt $(n=41)$. In 53 Anfragen fällt der Autorenname jedoch erst im Laufe des Gesprächs und häufig ungenau. In über $100 \mathrm{An}$ fragen erhält der Mitarbeiter nur Kontextinformationen zum Autor wie ein bekannter Krimiautor oder das Vorgängerbuch wird genannt. Weiterhin weisen Kunden auf einen Zeitbezug hin $(n=87)$ wie Veröffentlichungstermin (gerade erschienen oder vor einigen Jahren) oder einen Medienbezug z. B. Fernsehproduktion oder Buchbesprechung in der Presse. Hieraus lässt sich ableiten, dass Menschen mit unvollständigen Angaben umgehen können, sodass kein Aufwand in zusätzliche Informationsbeschaffung investiert werden muss. ${ }^{422}$ Suchmaschinen benötigen hingegen eindeutige Begriffe, um passende Ergebnisse liefern zu können.

\footnotetext{
${ }^{417}$ Vgl. Peters (2013), S. 230.

${ }^{418}$ Vgl. Mikkonen/ Vakkari (2016), S. 66.

${ }^{419}$ Vgl. Kroeber-Riel/ Gröppel-Klein (2013), S. 507 f.

${ }^{420}$ Vgl. Hinze et al. (2012), S. 6.

${ }^{421}$ Vgl. Buchanan/ McKay (2011), S. $271 \mathrm{f}$.

${ }^{422}$ Vgl. Buchanan/ McKay (2011), S. 276.
} 


\subsubsection{Stöbern in einer Menge von Büchern}

Stöbern als eine Form der Suche ohne Suchvorgabe bzw. Suchrichtung ist eine Aktivität, die nicht allein im Dienste der Tätigkeit Bücherlesen steht, sondern die für Kunden selbst wertvoll sein kann. Leser beschreiben diese Form des Stöberns als autotelische Tätigkeit, die dadurch gekennzeichnet ist, dass sie um ihrer selbst willen ausgeübt und als belohnend empfunden wird. ${ }^{423}$ Es lässt sich auch von ungeteiltem Interesse sprechen als einem motivational befriedigenden Zustand, der von positiven Gefühlen begleitet wird. ${ }^{424}$ Befragte Musikhörer geben an, teilweise so involviert zu sein, dass es ihnen schwerfällt mit dieser Tätigkeit aufzuhören. ${ }^{425}$ Insbesondere die Titel-zu-Menge-Verbindungen in Buchhandlungen eignen sich zum Stöbern. Rund $40 \%$ der Besucher von Osiander-Buchhandlungen verlassen das Geschäft im Durchschnitt ohne etwas gekauft zu haben. Riethmüller (Geschäftsführer Osiander) wertet dies als Anzeichen, dass Kunden den Laden nutzen, um zu Stöbern. ${ }^{426}$ Allgemein ist die häufigste Ursache für den Nichtkauf in einer Buchhandlung, dass eine Person dort Zeit verbringt, weil sie stöbern möchte. ${ }^{427}$ Schönbach spricht vom „Prinzip der zuverlässigen Überraschung “428, da Besucher eine geordnete Umgebung vorfinden, die sie von der Optionsanzahl nicht über-aber auch nicht unterfordert. ${ }^{429}$ Stöbern fördert den Eindruck von zufälligen Entdeckungen, die als wertvoll wahrgenommen werden. ${ }^{430}$

\subsection{Private Sphäre des Informationsmanagements}

\subsubsection{Bücher organisieren}

In einer qualitativen Befragung von Viellesern deckt Zeckert auf, dass gekaufte Bücher u. a. deshalb ungelesen bleiben, weil sie gekauft werden, damit eine Person für einen späteren Zeitpunkt etwas zu Lesen hat (Vorratshaltung). ${ }^{431}$ Abgesehen davon verschieben Personen den Erwerb von Büchern in die Zukunft bzw. werden im Alltag auf Bücher aufmerksam, die sie grundsätzlich interessant finden, aber nicht sofort lesen möchten. Um die Buchtitel nicht zu vergessen, verwenden Kunden Erinnerungshilfen z. B. in Form von Listen. Die Tätigkeit „Erstellen von Merklisten“

\footnotetext{
${ }^{423}$ Vgl. Thudt/ Hinrichs/ Carpendale (2015), S. 5; Musik vgl. Laplante/ Downie (2006), S. 2; vgl. Aellig (2004), S. 15.

${ }^{424}$ Vgl. Holodynski/ Oerter (2008), S. 542.

${ }^{425}$ Vgl. Laplante/ Downie (2006), S. 2; vgl. Aellig (2004), S. 15.

${ }^{426}$ Vgl. Graf (2014), ab 6. Min, https://www.youtube.com/watch?v=j9u7FD1elAA.

427 Vgl. Börsenverein (Hrsg.) (2015), S. 189; vgl. auch IfH (2010), S. 26.

428 Schönbach (2005), S. 347.

${ }^{429}$ Vgl. Schönbach (2005), S. 348; vgl. Schönbach (2009), S. 60 f.

${ }^{430}$ Vgl. Thudt/ Hinrichs/ Carpendale (2015), S. 6.

${ }^{431}$ Vgl. Zeckert (2007), S. 174 f., S. 178.
} 
dient also dem Ziel, Bücher zu organisieren. Da ein neues Objekt erzeugt wird, handelt es sich um eine vergegenständlichende Tätigkeit. In einer Befragung von Bibliothekskunden geben fünf Kunden in einem freien Kommentarfeld an, dass sie sich die Möglichkeit wünschen, eigene Medien- oder Merklisten erstellen zu können. ${ }^{432}$ Bei zufälligen Informationen zu interessanten Buchtiteln versuchen Vielleser sich diese zu merken oder führen Listen als Merkhilfe. ${ }^{433}$ Auch bei der E-BookLeser-Befragung von Henke aus dem Jahr 2002 fällt die hohe Wichtigkeit der Funktion Buchregal bzw. Leseliste auf (Rang 7 von 48). ${ }^{434}$ Nach welchem Organisationsprinzip dies geschieht, hängt vom Einsatzzweck der Liste ab, d. h. Bücher werden für den Anlass organisiert, zu dem sie genutzt werden z. B. Bücher zum Vorlesen für Kinder. ${ }^{45}$ Die Vergegenständlichung dient nicht nur als Erinnerungshilfe, sondern es geht Kunden auch darum, auf eine Sammlung außerhalb des Bestands zugreifen zu können, die nur Bücher enthält, die ihren Präferenzen entsprechen. Situativ erfolgt dann die Auswahl aus diesen Optionen. ${ }^{436}$ Merklisten bzw. persönliche Sammlungen reduzieren somit die Menge an Buchoptionen, sodass sich Kunden in einer (spontanen) Lesesituation schneller für ein Buch entscheiden können. Hanekop/Wittke argumentieren, dass die vorgeschaltete Selektion den Vorteil hat, dass ein Kunde beim Bücher suchen und auswählen Zeit und kognitive Energie spart und deshalb auch knappe Zeitfenster zum Bücherlesen nutzen kann. ${ }^{437}$ Somit besteht eine instrumentelle Verbindung zwischen den Aktivitäten „Merkliste erstellen“ und „Bücherlesen“. Bei gedruckten Büchern ist die Vorauswahl sogar unumgänglich, da eine Person nicht alle Bücher an Orte mitnehmen kann, an denen sie lesen möchte. ${ }^{438}$

\subsubsection{Informationen über eigene Aktivitäten organisieren}

In der E-Book-Leser-Befragung von Henke ist den Befragten zudem eine Aktivitätenhistorie wichtig, d. h. eine persönliche Übersicht über alle Handlungen wie Anmerkungen, Lesezeichen, etc. (Rang 12 von 48). ${ }^{439}$ Hinze et al. schlussfolgern aus einer Beobachtung von Kunden in wissenschaftlichen Bibliotheken, dass es für diese problematisch ist, wenn keine Vergegenständlichung von dem erfolgt, was sie bereits evaluiert, gelesen oder aufgerufen (identifiziert) haben. ${ }^{440}$ Bei der Rückgabe der Bücher muss eine Person versuchen, sich die Titel der Bücher zu merken. Eine solche Übersicht bzw. Funktion wird jedoch nicht nur im wissenschaftlichen oder

\footnotetext{
${ }^{432}$ Vgl. Fühles-Ubach/Brenner/Lorenz (2014), S. 72.

${ }^{433}$ Vgl. Ross (1999), S. 789.

${ }^{434}$ Vgl. Henke (2002), S. 10.

${ }^{435}$ Vgl. für Musik Weigl/ Guastavino (2011), S. 338; vgl. Cunningham/ Jones/ Jones (2004), S. 4.

${ }^{436}$ Vgl. Hanekop/ Wittke (2006), S. 133; vgl. für Musik Bentley/ Metcalf/ Harboe (2006), S. 5.

437 Vgl. Hanekop/ Wittke (2006), S. 127 f.

438 Vgl. Hanekop/ Wittke (2006), S. 127.

${ }^{439}$ Vgl. Henke (2002), S. 10.

${ }^{440}$ Vgl. Hinze et al. (2012), S. 9.
} 
beruflichen Kontext als nützlich wahrgenommen, sondern auch in einer Kundenbefragung in öffentlichen Bibliotheken geben vier Befragte im freien Kommentarfeld an, dass sie sich wünschen, ihre eigene Ausleihhistorie einsehen zu können. ${ }^{441}$ Die Alternative ist das mühselige Anlegen von Listen. Somit benötigen Kunden Personalisierung, also die Auslagerung der Handlungsdurchführung an Algorithmen, welche die Ergebnisse von Kundenaktivitäten wie angesehene Bücher, angewandte Lesestrategien (Lesezeichen, Markierungen etc.) (vgl. Kap. 6.3) und gelesene Bücher für den Kunden sichtbar dokumentieren und damit schnell auffindbar machen z. B. in Form einer Liste bzw. als Interaktionshistorie. Bei einer solchen Form der Personalisierung weist ein System aus Kundensicht eine hohe Responsiveness auf. Unter Responsiveness versteht man das Ausmaß, in dem ein System auf Kundeneingaben reagiert. ${ }^{442}$ Da sich die Informationsbereitstellung auf vorangehende Eingaben des Kunden bezieht, kann der Kunde die Aufeinanderbezogenheit seiner Mitteilungen und die des Systems unmittelbar erkennen und nachvollziehen. ${ }^{443}$ Abgesehen von dem Vorteil der Aufwandsreduktion konnte empirisch nachgewiesen werden, dass eine visualisierte Interaktionshistorie zu einem stärkeren Gefühl der Aufeinanderbezogenheit führt, das wiederum zu einem höheren Involvement beiträgt, das einen positiven Einfluss auf die Weiternutzungsabsicht hat. ${ }^{444} \mathrm{Im}$ empirischen Teil der Arbeit wird geprüft, ob Kunden sich beim Organisieren von Büchern/Informationen auf Möglichkeiten der Personalisierung und Customization beziehen.

Merkmale einer Dimension von Optimierungspotenzial: Customization bei Bücher organisieren, Personalisierung bei Bücher organisieren

\subsubsection{Informationen über Aktivitäten von anderen organisieren}

Leser sind zudem neugierig darauf zu erfahren, was andere lesen, weshalb sie z. B. in Bibliotheken nachschauen, welche Bücher gerade zurückgegeben wurden. ${ }^{445}$ Eine solche Funktion kann auch in ein Online-Angebot integriert werden, indem Kunden die Möglichkeit haben, die Statusmeldungen anderer Leser, aber auch einer Marke (z. B. Verlag) oder des Anbieters zu abonnieren. Hierbei handelt es sich um Customization, die wiederum eine Personalisierung zur Folge hat bzw. eine PullAktivität (Informationen selbst holen) wird durch eine Push-Aktivität (Informationen von einem Sender automatisch erhalten) ersetzt. ${ }^{446}$ Hierbei übernimmt ein Algorithmus die Durchführung der wiederkehrenden Prüfung von Profilen auf

\footnotetext{
${ }^{441}$ Vgl. Fühles-Ubach/Brenner/Lorenz (2014), S. 72.

${ }^{442}$ Vgl. Heeter (1989), S. 223; vgl. Quiring/ Schweiger (2006), S. 12.

${ }^{443}$ Vgl. Quiring/ Schweiger (2006), S. 18; vgl. Sundar et al. (2016), S. 597; vgl. Sundar et al. (2015), S. 61; vgl. Neuberger (2007), S. 38.

${ }^{444}$ Vgl. Sundar et al. (2016), S. 613 f., S. 616 ff.

445 Vgl. Ross (1999), S. 797.

${ }^{446}$ Vgl. Hautzer/ Lünich/ Rössler (2012), S. 29, S. 35 f.
} 
Veränderungen und auf potenziell nützliche Hinweise. Indem Kunden Informationen über andere Kunden beziehen, die einen ähnlichen Lesegeschmack haben, profitieren sie davon, dass sie auf Bücher aufmerksam werden ohne selbst Bücher zu suchen. ${ }^{447}$ Außerdem hat die „passive Buchempfehlung“ für die Empfänger den Vorteil, dass der Sender keine Reziprozität erwartet. Diese „unpersönliche“ Art und Weise, persönliche Information zu erhalten, ist „less socially demanding“448 und erfordert z. B. kein Feedback im Sinne von „Dankeschön“. Darüber hinaus können sich Kunden mittels Push-Funktion z. B. über Neuerscheinungen informieren lassen. Informationen über Trends/Novitäten sind für Kunden in Buchhandlungen von mittlerer Wichtigkeit ${ }^{449}$ und $73 \%$ der Bibliothekskunden finden es wichtig, Tipps und Empfehlungen zu erhalten, was Kinder gut lesen können. ${ }^{450}$

\section{6 Öffentliche Sphäre des Informationsmanagements bei Online-Angeboten}

Das Gefühl der Agency ist mit der Funktion der Customization nicht vollständig erklärt, da Kunden bei Online-Angeboten nicht nur Anpassungen für sich selbst vornehmen, sondern auch Gatekeeper und Produzent für andere sein können. ${ }^{451}$ Die Aspekte Publizität und Erhalt von Rückmeldung führen vermutlich zum starken Wirksamkeitserleben. ${ }^{452}$ Darauf aufbauend ordnet Sundar Aktivitäten danach, in welchem Ausmaß sie das Gefühl von Agency verstärken können: ${ }^{453}$

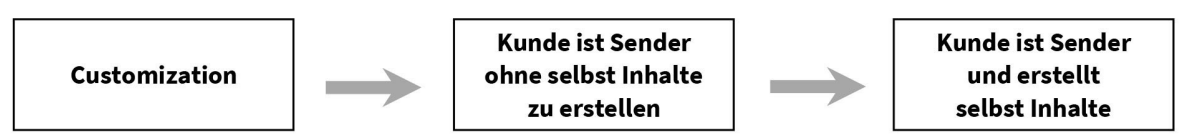

Abb. 6.6 Rangfolge von Aktivitäten nach Einflussstärke auf das Gefühl der Agency (Quelle: Eigene Erstellung)

Die Kundenaktivitäten, bei denen es um Pflege, Bewerten und Weitergabe von Inhalten geht, können anderen Kunden als Inspiration sowie als Navigations- und Evaluationshilfe dienen. Werden die Kundenaktivitäten zusammengenommen betrachtet, kann man von einem Prozess des gemeinsamen Filterns und Organisierens

\footnotetext{
447 Vgl. für Musik Håkansson/ Rost/ Holmquist (2007), S. 325.

448 Vgl. Håkansson/ Rost/ Holmquist (2007), S. 327.

449 Vgl. Schwiering (2011), S. 8.

${ }^{450}$ Vgl. Institut für Demoskopie Allensbach (Hrsg.) (2016), S. 13.

${ }^{451}$ Vgl. Sundar et al. (2015), S. 57; vgl. Sundar (2008), S. 60.

${ }^{452}$ Vgl. Klimmt/ Blake, S. 76.

453 1. Customization 2. Curation of content 3. Creation of content (vgl. Sundar et al. (2015), S. 65).
} 
von Inhalten sprechen, der die Auswahl von Büchern unterstützt. ${ }^{454}$ Hier findet kein Gatekeeping durch die Betreiber des Angebots statt, sondern durch die Kunden selbst - entweder im Kollektiv (z. B. automatisch generierte Popularitätslisten) oder durch eine einzelne Person verursacht (z. B. Inhalt auf einer Skala bewerten, Aktivitäten automatisch mit anderen teilen, Rezension schreiben). ${ }^{455}$ Die Kundenaktivitäten, die in dieser Hinsicht hilfreich sind, müssen daher nicht immer vergegenständlichend sein, sondern Personen können auch durch unabsichtliche Rückmeldungen zum Organisations- und Filterprozess beitragen ${ }^{456}$, indem sie Inhalte und Bücher aufrufen und diese Verhaltensdaten aggregiert dargestellt werden. Solche Datenspuren entstehen quasi als Nebenprodukt aneignender Aktivitäten. ${ }^{457}$

\subsubsection{Voraussetzungen für das gemeinsame Informationsmanagement}

Eine Voraussetzung für das gemeinsame Filtern ist, dass der Anbieter die Bedingungen schafft, damit die Ergebnisse der Aktivitäten wahrnehmbar sind. Hierfür kann er einerseits Signale in Form von automatisch erzeugten Statistiken integrieren und andererseits Kundeneingaben als 1:1-Umsetzung darstellen (vgl. Kap. 5.5). ${ }^{458}$ Somit lässt sich festhalten: „Jede Handlung im Internet kann anderen Personen dann als Navigationshinweis dienen, wenn ihnen mindestens eine Information über diese Handlung technisch zur Verfügung steht. “459

Zwei andere Voraussetzungen betreffen die Kundenseite. Allgemein wird die Entscheidung darüber, eine Aufgabe zu erledigen, von dem Eindruck einer Person beeinflusst, ein bestimmtes Ergebnis durch eigenes Tun verursachen zu können. ${ }^{460}$ Die Annahme fällt negativ aus, wenn sie sich der Aufgabe nicht gewachsen sieht, obwohl sie glaubt, dass sie ein Ergebnis bewirkt. ${ }^{461}$ Für die Einschätzung einer Person - eine Aufgabe erfolgreich erledigen zu können - hat Bandura den Begriff der Selbstwirksamkeitserwartung (self-efficacy) geprägt und diese von der Ergebniserwartung abgegrenzt. ${ }^{462}$ Sie beeinflusst direkt, ob Personen Web 2.0- Aktivitäten ausüben. ${ }^{463}$ Damit Kunden z. B. in den Merk- und Leselisten anderer Kunden stöbern können, müssen diese öffentlich zugänglich sein. Hierfür ist die Bereitschaft zur Selbstoffenbarung nötig. Selbstoffenbarung meint jede Information über das Selbst, welche eine Person anderen mitteilt. ${ }^{464}$ Es sind Informationen, die eine

\footnotetext{
${ }^{454}$ Vgl. Hautzer/ Lünich/ Rössler (2012), S. 18: vgl. auch Jers (2012), S. 58.

${ }^{455}$ Vgl. Sundar (2008), S. 66.

${ }^{456}$ Vgl. Heeter (1989), S. 231.

457 Vgl. Hautzer/ Lünich/ Rössler (2012), S. 23 f., S. 30 f.

${ }^{458}$ Vgl. Schweiger/ Quiring (2006), S. 102, S. 111; vgl. Hautzer/ Lünich/ Rössler (2012), S. 29.

459 Vgl. Hautzer/ Lünich/ Rössler (2012), S. 23.

${ }^{460}$ Vgl. Rheinberg/ Vollmeyer (2012), S. 137.

461 Vgl. Rheinberg/ Vollmeyer (2012), S. 138.

${ }^{462}$ Vgl. Bandura (1977/ 1982), S. 193; vgl. auch Rheinberg/ Vollmeyer (2012), S. 139.

${ }^{463}$ Vgl. Jers (2012), S. $324-327$, S. 329.

${ }^{464}$ Vgl. Taddicken/Schenk (2011), S. 320.
} 
Wer darf deine Bücher sehen?

Die Liste deiner bewerteten Bücher

Die Bücher auf deiner Wunschliste

Deine vorgemerkten Butcher

Bucher, die du besitzt

Liste der Bücher, an denen ou kein Interesse hast

\begin{tabular}{|c|c|}
\hline Readgeek-Mitglieder & $\triangle$ \\
\hline $\begin{array}{l}\text { Nur ich } \\
\text { Freunde } \\
\text { Freunde von-Freunden } \\
\text { Readgeek-Mitglieder }\end{array}$ & \\
\hline 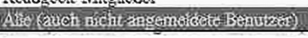 & \\
\hline Alle (auch nicht angemeldete Bemutzet) & 7 \\
\hline
\end{tabular}

Abb. 6.7 Customization durch Privatsphäre-Einstellungen (Quelle: Screenshot Readgeek, 06. 09. 2016)

Person als sehr privat oder weniger privat einstuft, die aber immer Teil ihrer Privatsphäre sind. Privatsphäre meint die ,selektive Kontrolle des Zugangs zum Selbst“. ${ }^{465}$ Im Jahr 2014 stimmten rund $3 \%$ und im Jahr 2015 rund 4 \% der E-BookLeser der Aussage zu, dass sie eigene Leselisten (im Internet) veröffentlichen. ${ }^{466} \mathrm{Da}$ die Bereitschaft zur Selbstoffenbarung unterschiedlich ausgeprägt ist bzw. sich ändern kann, benötigen Kunden die Möglichkeit, ihre Privatsphäre zu schützen (vgl. Abb. 6.7). ${ }^{467}$

Das Verhalten, freiwillig Informationen über sich preiszugeben, wird vermutlich u. a. durch inneren Druck zur Reziprozität beeinflusst, weil auch andere Kunden Informationen über sich offenbaren ${ }^{468}$ und eine Person z. B. das Stöbern in den Merklisten anderer Kunden nützlich findet.

\subsubsection{Informationen bereitstellen ohne eigene Inhalte zu erstellen}

Bei den nachfolgenden Aktivitäten sind Kunden selbst Informationsgeber für andere Kunden (vgl. Abb. 6.6). Sie nutzen die Möglichkeit, Informationen mit dem System auf einfache Weise erzeugen zu können. ${ }^{469}$ Hierbei sind sich Kunden den vielen potenziellen Empfängern ihrer Nachrichten bewusst, was in ihnen das Gefühl hervorrufen kann, die eigene Agency hätte eine größere Wichtigkeit. ${ }^{470}$ Die Aktivitäten bieten speziell den Vorteil, dass Kunden die Rolle des Senders einnehmen können, ohne die Hürde überwinden zu müssen, selbst Inhalte zu erstellen.

Oestreicher-Singer et al. fanden heraus, dass die Anzahl ausgeübter Aktivitäten, Spendenbereitschaft und Weiternutzungsabsicht in Bezug auf ein Angebot (Videodienst) höher ausfällt, wenn die verschiedenen Aufgaben, welche die Probanden erledigen sollen, in einer geordneten Reihenfolge präsentiert werden. ${ }^{471}$ Eine ge-

\footnotetext{
${ }^{465}$ Vgl. Taddicken/Schenk (2011), S. 322.

${ }^{466}$ Vgl. Bitkom (2014), S. 11; vgl. Bitkom (2015), S. 12.

467 Vgl. für Musik Weigl/ Guastavino (2011), S. 338.

${ }^{468}$ Vgl. Taddicken/Schenk (2011), S. 329.

${ }^{469}$ Vgl. Jers (2012), S. 82; vgl. Heeter (1989), S. 224.

${ }^{470}$ Vgl. Sundar (2008), S. 68.

${ }^{471}$ Vgl. Zalmanson/ Oestreicher-Singer (2015), S. 19.
} 
ordnete Reihenfolge zeichnet sich dadurch aus, dass diese sich an der durchschnittlichen Häufigkeit orientiert, mit der die jeweiligen Aktivitäten ausgeführt werden, und die mit der beliebtesten Aktivität beginnt (hier: 1. Bewertungsskala, 2. Mögen-/NichtMögen-Button, 3. Kommentarfunktion, 4. Taggen). ${ }^{472}$ Die Autoren vermuten daher, dass ein gradueller Anstieg des Schwierigkeitsgrads bei den Aufgaben wichtig ist. ${ }^{473}$ Auch Dahl/Moreau bestätigen empirisch, dass bei einer Aufgabe, die auf die Erfahrenheit einer Person abgestimmt ist, die Bedürfnisbefriedigung am stärksten erlebt wird, was wiederum zu einem größeren Vergnügen bei der Aktivität führt. ${ }^{474}$ Die Voraussetzung hierfür ist, dass die nötige Kreativität zur Bewältigung der Aufgabe entsprechend der Erfahrenheit beschränkt wird. Eine eher unerfahrene Person benötigt mehr Beschränkungen und mehr Anleitung als eine eher erfahrene Person. ${ }^{475}$

- Buch, Rezensionen/Kommentare anderer Kunden etc. bewerten

Hierfür integrieren Anbieter Bewertungsoptionen (z. B. 5-Sterne-Skala) oder einen Button („Gefällt mir“ bzw. „Gefällt mir nicht“) in die Benutzungsoberfläche. Die Trennung der Bewertungsfunktion von der kreativeren Aufgabe, Inhalte zu erstellen, wird bei Readfy technisch unterbunden. Um die Bewertungsskala nutzen zu können müssen die Personen gleichzeitig etwas schreiben. ${ }^{476} \mathrm{Da}$ sich der Schwierigkeitsgrad der Aufgaben aus Kundensicht unterscheidet, ist eine denkbare Folge, dass die Bewertungsfunktion weniger genutzt wird, weil Kunden zusätzlich die Aufgabe bewältigen müssen, etwas Eigenes zu formulieren.

- Status-Sharing (Statusmeldungen veröffentlichen)

Kunden können andere durch die Funktion des Status-Sharing über ihr Tun informieren. ${ }^{477}$ Hierzu zählt die Veröffentlichung von buchbezogenen Aktivitäten z. B. welches Buch ein Kunde gerade liest, welches Buch ein Kunde auf seine Merkliste setzt etc. Die Kundenaktivitäten sind im Activity Stream anderer Kunden sichtbar, wenn diese die Statusmeldungen abonniert haben. Die Statistik zu den Abonnenten informiert den Kunden wiederum über die Empfängerzahl. Darüber hinaus können Kunden ihren Kontakten in anderen Online-Diensten mitteilen, was sie gerade tun. Hierfür muss der Anbieter Schnittstellen zu sozialen Netzwerken oder Microblogging-Diensten vorsehen. ${ }^{478}$ Bei dieser Art der Informationsbereitstellung handelt es

\footnotetext{
${ }^{472}$ Vgl. Zalmanson/ Oestreicher-Singer (2015), S. 11.

473 Vgl. Zalmanson/ Oestreicher-Singer (2015), S. $27 \mathrm{f}$.

${ }^{474}$ Vgl. Dahl/ Moreau (2007), S. 364, S. 366 f.

475 Vgl. Dahl/ Moreau (2007), S. 364, S. 367.

476 Prüfung der Funktion in der Readfy-App für Android am 25. 08. 2016.

477 Vgl. Böhringer/ Gerlach (2010), S. 2.

${ }^{478}$ Vgl. Schmidt (2011), S. 65.
} 
sich um passives Mitteilen ${ }^{479}$ bzw. die Veröffentlichung erfolgt automatisch, sodass für die Zugänglichmachung kein Aufwand investiert werden muss. Die Veröffentlichung ist - wie bereits erwähnt - mit der Bereitschaft verbunden, Informationen über sich preiszugeben. Darüber hinaus können Kunden den Wunsch haben, bestimmte Bücher, die sie lesen oder die sie auf eine Merkliste setzen, geheim zu halten, worauf Tamblyn (CEO Rakuten Kobo) aufmerksam macht: „Wir haben beobachtet, dass die Leute ein privates und ein öffentliches ,Leseleben“ haben. Wenn man jemandem das Gefühl gibt, dass alles, was er liest, auch automatisch mit anderen geteilt wird, wird man diesen Kunden wohl nicht mehr lange als Kunden haben. Man muss den Lesern also die Möglichkeit geben, selektiv nur das zu teilen, was sie anderen weitergeben möchten. “480 Da Kunden nicht alle Bücher und Aktivitäten bekannt geben möchten, benötigen sie die Option, wahlweise den Automatismus der Zugänglichmachung zu unterbrechen. Für das private Lesen bzw. die private Angebotsnutzung kann der Anbieter einen Modus implementieren, in dem, wenn Kunden ihn aktivieren, Aktivitäten zeitweise nicht veröffentlicht werden (Customization). Beim Musikstreaming-Dienst Spotify erlaubt dies die Funktion „Private Session“, die vom Anbieter so beschrieben wird: „Ob Du in musikalischen Jugendsünden stöbern oder einfach mal nur für Dich Musik hören möchtest: Die private Session ermöglicht Dir eine Auszeit.“481

- Link zu einem Buch oder einer Textstelle weiterleiten

Vom passiven Mitteilen ist das aktive Mitteilen von Informationen an Personen oder Kontakte in Netzwerk abzugrenzen. ${ }^{482}$ Kunden können den Link zu einem Buch oder einer Textstelle per EMail, Instant Messaging oder über ein soziales Netzwerk verteilen. In einer qualitativen Studie zeigt sich, dass Personen im Umgang mit Freunden eine aktive Form des Teilens bevorzugen. ${ }^{483}$

In diesen Fällen haben Mitteilungen eine Beziehungsfunktion z. B. können sie Nähe ausdrücken, Vorlieben deutlich machen oder der Sender kann durch eine Empfehlungen zeigen, dass er die andere Person gut kennt. ${ }^{484}$ Die Nachrichten werden vom Empfänger auch als Erinnerungshilfe genutzt, um einen Inhalt im Alltag nicht zu vergessen. ${ }^{485}$ Darüber hinaus kann die Weiterleitung eine Lesestrategie sein (vgl. Kap. 6.3). Zum Beispiel wenn der Sender hofft, sich mit anderen über das Gelesene austauschen zu können. Dies soll helfen, die eigene emotionale Betroffenheit, die durch die Lektüre verursacht wurde, zu regulieren. ${ }^{486}$ Oder wenn

\footnotetext{
${ }^{479}$ Vgl. Haupt/Grünewald, S. 100.

480 Bei Tablets gibt es kaum Differenzierungsmöglichkeiten (2014), buchreport.de.

${ }^{481}$ Spotify, https://support.spotify.com/de/using_spotify/share_music/how-to-enable-private-listening/

482 Vgl. Haupt/Grünewald, S. 100.

${ }^{483}$ Vgl. Håkansson/ Rost/ Holmquist (2007), S. 326.

${ }^{484}$ Vgl. für Musik Haupt/ Grünewald, S. 102.

${ }^{485}$ Vgl. Håkansson/ Rost/ Holmquist (2007), S. 322.

${ }^{486}$ Vgl. Charlton/ Pette (1999, S. 109, S. 111.
} 
Kunden die Weiterleitungsfunktion nutzen, um ihre Belesenheit zu demonstrieren. ${ }^{487}$ Im Jahr 2014 gaben $3 \%$ der E-Book-Leser an, bereits einmal Textstellen markiert und mit anderen geteilt zu haben z. B. über soziale Netzwerke und im Jahr 2015 erwähnten $5 \%$, dass sie anderen im Internet mitteilen, was sie lesen. ${ }^{488}$

\subsubsection{Informationen bereitstellen und eigene Inhalte erstellen}

Die nachfolgenden Aktivitäten unterscheiden sich von den Vorangehenden, weil Kunden selbst herausfinden müssen wie sie ihre Gedanken sinnvoll ausdrücken können (vgl. Abb. 6.6). Durch diese vergegenständlichenden Aktivitäten erschaffen sie neue (eigene) Objekte. Wie lange diese Objekte in der Welt existieren hängt u. a. von den Entscheidungen des Anbieters ab. Hierzu gehört z. B. das Verschlagworten von Büchern („Tagging“). Diese Tätigkeit unterstützt den sozialen Filterprozess. ${ }^{489}$ Des Weiteren fallen in diese Kategorie die Aktivitäten „Bücher in vergleichsweise wenigen Worten kommentieren“ oder umfangreichere Beiträge verfassen wie „eine Rezension schreiben“ oder „einen Blogeintrag erstellen“. Wie bereits dargelegt, erfordert die höhere Freiheit im Ausdruck mehr Kreativität. Die Möglichkeit der freien Eingabe wird vermutlich gewählt, wenn eine Person stark involviert ist oder sie ihre Identität ausdrücken möchte. ${ }^{490}$ Inhalte wie Rezensionen und Schlagworte werden jedoch nicht von allen Kunden im Auswahlprozess eingesetzt. Bei einer Befragung von Bibliothekskunden - nach der testweisen Integration von $u$. a. Rezensionen und Tagclouds in die Benutzungsoberfläche des Online-Katalogs - geben 66 \% der Kunden an, Rezensionen zumindest wahrgenommen zu haben. Von diesen haben $36 \%$ Rezensionen gelesen, aber nur rund $40 \%$ empfinden Rezensionen als mindestens eher hilfreich. ${ }^{491}$ Die häufigsten Kritikpunkte sind eine schlechte Qualität (z. B. fehlende Aussagekraft) und eine zu geringe Anzahl pro Titel. ${ }^{492}$ Diese Aussagen behalten ihre Gültigkeit auch bei der allgemeinen Beurteilung der Nützlichkeit von Rezensionen im Internet. ${ }^{493}$ Die Tagclouds wurden von $51 \%$ der Bibliothekskunden wahrgenommen, aber nur von $17 \%$ der Kunden genutzt. Zudem gaben nur knapp $30 \%$ an, die Tags als mindestens eher hilfreich zu empfinden. ${ }^{494}$ Am häufigsten wurde kritisiert, dass die verlinkten Buchtitel unpassend waren oder ein Begriff nicht zum getaggten Buch gepasst hat. ${ }^{495}$ Darüber hinaus erschließt sich der Sinn der

\footnotetext{
${ }^{487}$ Vgl. Charlton/ Pette (1999, S. 111.

${ }^{488}$ Vgl. Bitkom (2014), S. 11; vgl. Bitkom (2015), S. 12.

489 Vgl. Hautzer/ Lünich/ Rössler (2012), S. 34.

490 Vgl. Schweiger/ Quiring (2006), S. 112.

${ }^{491}$ Vgl. Fühles-Ubach/Brenner/Lorenz (2014), S. 66 ff.; n= 1.154 Kunden aus 19 deutschen Bibliotheken.

492 Vgl. Fühles-Ubach/Brenner/Lorenz (2014), S. 67.

493 Vgl. Fühles-Ubach/Brenner/Lorenz (2014), S. 68.

${ }^{494}$ Vgl. Fühles-Ubach/Brenner/Lorenz (2014), S. 64.

${ }^{495}$ Vgl. Fühles-Ubach/Brenner/Lorenz (2014), S. 64 f.; Englischsprachige Schlagworte wurden mithilfe von LibraryThing-Nutzern und Wikipedia ins Deutsche übersetzt (vgl. Fühles-Ubach/ Brenner/ Lorenz (2014), S. 11).
} 
Tagging-Funktion nicht: 134 Bibliothekskunden geben an, dass sie die Funktion überflüssig finden. ${ }^{496}$ An dieser Stelle ist der Hinweis wichtig, dass die häufigsten Strategien im Auswahlprozess die Suchanfrage, das Explorieren von Suchtreffern (Scanning) und das Prüfen von Buchdetailseiten (Evaluation) sind ${ }^{497}$ Demzufolge ist die Mikrostrategie „Verbindungen folgen“ bei der Suche in Online-Katalogen (noch) nicht stark verbreitet. „Dieses Verhalten entspricht der lange Jahre einzigen Funktion des Katalogs und legt somit die Interpretation nahe, dass es sich hier um erlerntes Verhalten handelt, das nicht zwingend dem tatsächlichen Informationsbedarf der Nutzer entspricht. “498 Kunden zeigen also je nach Gestaltung ein unterschiedliches Navigationsverhalten. In Räumen werden Verbindungen auf natürliche Weise wahrgenommen und genutzt. Die im Regal nebeneinander angeordneten Bücher sind eine Affordance, mit der sich das Stöbern ohne Nachdenken umsetzen lässt. Bei der Erläuterung der Mikrostrategie „Verbinden“ (vgl. Kap. 6.4.3) hat sich gezeigt, welche Navigationswerkzeuge zum Ausprobieren dieser Suchstrategie anregen können. Erst wenn Kunden diese Werkzeuge - z. B. die Stichwortsuche über Tags - selbst nutzen, werden sie es vermutlich überhaupt in Betracht ziehen, selbst Bücher zu verschlagworten.

496 Vgl. Fühles-Ubach/Brenner/Lorenz (2014), S. 65.

497 Vgl. Mikkonen/ Vakkari (2016), S. 66; vgl. Thudt/ Hinrichs/ Carpendale (2015), S. 1, S. 4.

498 Vgl. Fühles-Ubach/ Brenner/ Lorenz (2014), S. 61. 


\section{Inhaltsanalyse von Kundenkommentaren bei Readfy und Skoobe}

\subsection{Kurzbeschreibung der untersuchten Angebote}

Die Vermietungsangebote Readfy und Skoobe nutzen beide das Abrufprinzip. Bei den Bereitstellungsverfahren können Kunden zwischen Web-Cloud und Download (Offline-Lesen) wählen, wobei die Bücher nur mit Anbietersoftware gelesen werden können. Bei Skoobe kann ein Kunde entweder 24 Stunden offline Bücher lesen oder gegen einen Aufpreis 30 Tage lang. Es handelt sich jeweils um einen Flatrate-Tarif mit einer eingeschränkten Menge an Büchern, die gleichzeitig parallel offline gelesen werden kann. Bei Readfy können Kunden online kostenlos lesen ohne jegliche Beschränkungen. Um offline lesen zu können wird ein transaktionsabhängiges Entgelt fällig. Die Mietdauer liegt bei 30 Tagen. Beim Online-Lesen wird sowohl reizstarke (Interstitial) als auch reizarme Werbung (Banner am Seitenrand) eingeblendet (siehe Abb. 7.1)

Beim Offline-Lesen werden keine Werbemittel angezeigt. Die Möglichkeit der transaktionsabhängigen Miete bestand für Kunden von Readfy im Untersuchungszeitraum noch nicht. Das Lesen im Web-Browser ist jeweils nicht möglich. Offiziell
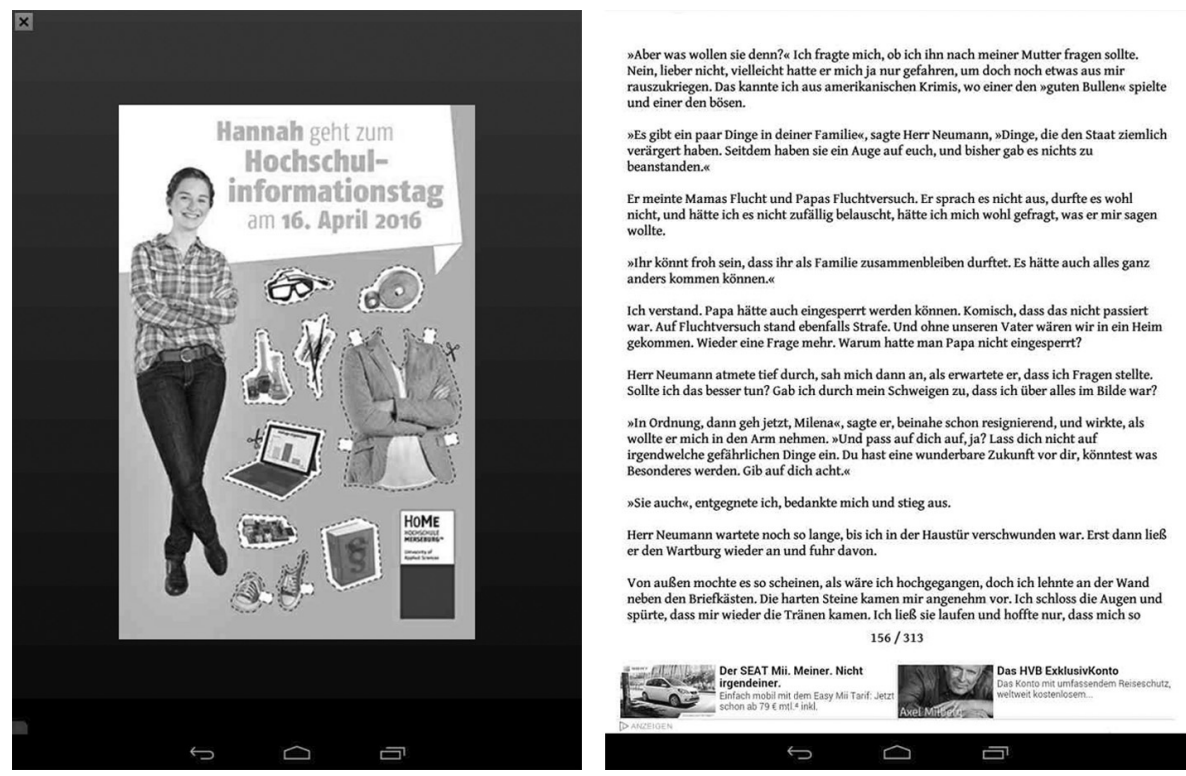

Abb. 7.1 Beispiele für Werbeformen in einer softwarebasierten Benutzungsschnittstelle (Quelle: Screenshots Readfy, 15. 04. 2016/ 02.09. 2016) 
werden von beiden Anbietern nur Geräte mit den Betriebssystemen iOS (Apple) oder Android (Google) unterstützt. Beide Angebote sind als App umgesetzt, ohne Varianten für Desktop-PC oder Laptop, und es lassen sich mehrere Geräte unter einem Konto registrieren. In beiden Angeboten können Kunden Bücher lesen, Bücher suchen, Listen anlegen, Rezensionen/ Kommentare schreiben, Bücher bewerten (5er-Skala), Textstellen/Bücher weiterleiten und für einige Aktivitäten auf eine Interaktionshistorie zurückgreifen. Nicht vorhanden sind TagClouds bzw. Tags als Navigationswerkzeug bzw. die Aktivität Taggen und eine Abo-Funktion für Aktivitäten anderer Kunden bzw. die Aktivität StatusSharing. Nur bei Readfy können Kunden auf öffentliche Leselisten anderer Kunden zugreifen. ${ }^{499}$

\subsection{Untersuchungsziele}

Die Untersuchung hat sich zum Ziel gesetzt, folgende Frage zu beantworten:

1. Frage: Erwähnen Kunden die theoretisch abgeleiteten Qualitätsmerkmale der Bereitstellung von Büchern? Welche Merkmale werden am häufigsten genannt?

1.1 Unterfrage: Sind eine fehlende Verfügbarkeit von gesuchten Büchern und ein hohes Anspruchsniveau hemmende Faktoren in Bezug auf die Übereinstimmung des Buchbestands mit Kundenpräferenzen?

1.2 Unterfrage: Wird Werbung als belastende Störung empfunden? Hängt das Ausmaß der wahrgenommenen Störung vom Werbemittel ab (reizarm/reizstark)? Gibt es Hinweise darauf, dass Kunden Werbevermeidungsstrategien anwenden?

2. Frage: Welchen buchbezogenen Aktivitäten gehen Kunden in den Angeboten nach? Welche Aktivitäten werden am häufigsten genannt?

3. Frage: Besteht Optimierungspotenzial bei den Kundenaktivitäten, dh. benötigen bzw. wünschen Kunden sich Möglichkeiten der individuellen Anpassung (Customization) und Möglichkeiten zur Personalisierung? Wenn ja, welche?

4. Frage: Lassen sich bei der Urteilsbildung des Kunden Vergleichsprozesse nachweisen? Wenn ja, lassen sich konkret Urteile im Hinblick auf das Sparpotenzial eines Angebots und das Kosten-Buchlesemengen-Verhältnis feststellen?

\subsection{Beschreibung der Methode}

Die Voraussetzung für die Beantwortung der Fragen ist, dass Personen untersucht werden, die bereits Erfahrungen mit dem Angebot gesammelt haben. ${ }^{500}$ Weiterhin muss aus den Kundenaussagen zur Beantwortung von Frage 1 abgeleitet werden können, dass sie tatsächlich Qualitätsbewertungen (wertvoll/weniger wertvoll) wi-

\footnotetext{
499 Die Autopsie der Aktivitäten und Angebotsmerkmale erfolgte am 17.05. 2016 mit einem AndroidBetriebssystem.

${ }^{500}$ Vgl. Wolf (2014), S. 151.
} 
derspiegeln. Hierfür wird der Untersuchung ein Erwartungs-Bestätigungs-Modell zugrunde gelegt. Demnach bilden Kunden Erwartungen und können im Anschluss an die Nutzung aufgrund der erlebten Performance bewerten, in welchem Ausmaß ihre Erwartungen bestätigt wurden. Oliver spricht von der Nichtbestätigung (Disconfirmation) als eine „psychological interpretation of an expactation-performance discrepancy (Abweichung zwischen Erwartungs- und Performance-Level; Anm.). Consumers would describe this concept in terms of the performance of a product or service being better or worse than expected. ${ }^{“ 501}$ Wenn Kunden sich beim Verfassen von Kommentaren an Erlebnisse bei der Angebotsnutzung erinnern oder an solche, die damit in Zusammenhang stehen, kann angenommen werden, dass sie durch die Schilderung von Ereignissen ihre Bewertungsstrukturen offenlegen. ${ }^{502}$ In den Urteilen kommt der aktuelle Bestätigungsgrad als Ergebnis der Angebotsnutzung zum Ausdruck, sodass sie einen Rückschluss auf die Erwartungen der Kunden zulassen.

Bei der Untersuchung werden quantitative und qualitative Analyseschritte kombiniert. Eine ausschließliche Reduktion auf eine Variante erscheint nicht sinnvoll, denn einerseits hilft die quantitative Analyse, große Textmengen zu bewältigen, d. $h$. Komplexität zu reduzieren. Andererseits erlaubt es die qualitative Analyse, indem sie Komplexität zulässt, die Kundenlogik nachzuvollziehen (Angebotsnutzung verstehen). ${ }^{503}$ Um Daten erheben zu können, wird die Inhaltsanalyse als Instrument eingesetzt. Bei dieser Methode wird jeder Kategorie, die identifiziert werden kann, ein Zahlenwert zugeordnet (Codierung). Die Zahlenwerte bilden solche Daten, die später quantitativ ausgewertet werden können. ${ }^{504}$ Die Inhaltsanalyse ist somit eine Suchstrategie. ${ }^{505}$ Eine quantitative Analyse ist bei Frage 1und 2 wichtig, da es um die Feststellung, ob und wie viele Personen ein Verhalten zeigen. Die Häufigkeit des Auftauchens einer Kategorie deutet auf eine allgemein große Bedeutung für Kunden von Vermietungsangeboten für E-Books hin. ${ }^{506}$ Im Hinblick auf das Datenniveau wird hauptsächlich eine Nominalskala (vorhanden/nicht vorhanden) und selten eine Ordinalskala (Ausprägung wichtig/unwichtig, positiv/negativ) verwendet. ${ }^{507}$ Die Einschränkung auf weitestgehend dichotome Kategorien ist angesichts der relativ hohen Anzahl an Kategorien und Kommentaren sinnvoll. ${ }^{508}$ Zudem ist es weitgehend das Ziel herauszufinden, ob ein Aspekt thematisiert wird. Analysen auf Basis der Nominalskala sind qualitative Analysen. ${ }^{509}$ Um 1.1, 1.2, 3 und 4 beantworten zu können, muss qualitative Analyse des Materials erfolgen. Mithilfe einer quantitativen Analyse wurde es hierfür auf relevante Textstellen eingegrenzt.

\footnotetext{
501 Oliver (2015), S. 22.

${ }^{502}$ Vgl. Fließ/ Wittko/ Schmelter (2012), S. 170.

${ }^{503}$ Vgl. Mayring (2015), S. 19.

${ }^{504}$ Vgl. Früh (2015), S. 31 f., S. 67, S. 84.

505 Vgl. Früh (2015), S. 67.

506 Vgl. Mayring (2015), S. 53.

507 Vgl. Früh (2015), S. 34; vgl. Mayring (2015), S. 18.

508 Vgl. auch Wolf (2014), S. 258.

${ }^{509}$ Vgl. Mayring (2015), S. 18.
} 


\subsection{Festlegung des Materials und Analyse des Entstehungskontextes}

Ausgewählt wurden die Nutzerkommentare zu den Apps von „Readfy“ und „Skoobe“ im Google Play Store (https://play.google.com/store/apps in der Kategorie Bücher \& Nachschlagewerke) und im Amazon App Store (https://www.amazon.de in der Kategorie Spiele und Apps/Bücher \& Reader). Der Untersuchungsgegenstand befindet sich auf der Produktseite der App im jeweiligen Shop in einem standardisierten Bereich mit der Bezeichnung „Bewertungen“ (Google Play Store) bzw. „Kundenrezensionen“ (Amazon App Store). Die Kommentare beziehen sich auf alle App-Versionen im Auswertungszeittraum. Die Kopien der Nutzerkommentare wurden am 01.03. 2015 (Skoobe-App) und am 04.10.2015 (Readfy-App) erstellt und in einer Word-Datei gespeichert. Der Auswertungszeitraum bei Skoobe entspricht 14 Monaten (01.01.2014-28.02.2015) und der bei Readfy 16 Monaten (01.11. 2014 - 29.02. 2016), um ausreichend viel Datenmaterial zu gewinnen. Insgesamt handelt es sich um eine Auswahl aus einer Grundgesamtheit (alle Kunden von Vermietungsangeboten für E-Books im deutschsprachigen Raum) (vgl. Anhang A).

Bei der Entstehungssituation geht es um die Frage, unter welchen Bedingungen das sprachliche Material produziert wurde. ${ }^{510}$ Das Material kam ohne Einfluss der Bearbeiterin zustande und ist Ausdruck von kognitiven Bewertungen und Emotionen von Personen, welche die jeweilige App mindestens einmal genutzt oder dies versucht haben. Um einen Kommentar in einem Appstore verfassen zu können, muss die App (zumindest im Appstore von Google) auf einem Gerät installiert worden sein. Der Kommentar richtet sich an die Angebotsbetreiber und/oder die Öffentlichkeit. Es ist möglich, dass sich die Verfasser der Kommentare gegenseitig in ihrem Urteil beeinflussen, da die Mitteilungen öffentlich sind. Zur Demografie der Verfasser lassen sich keine zuverlässigen Angaben machen. Die Anonymität und die Gefahr von fingierten Kommentaren können zu Verzerrungen führen. Ein großer Umfang der Stichprobe soll hier ausgleichend wirken ${ }^{511}$, jedoch bleibt zu bedenken, dass einzelne Kategorien vermutlich nur selten codiert werden.

\subsection{Beschreibung der Analyseeinheit und Ablauf der Untersuchung}

Die Analyseeinheit in dieser Arbeit bildet der Kommentar eines Kunden (zu den Definitionskriterien vgl. Anhang A). Unter der Analyseeinheit versteht man „die

\footnotetext{
${ }^{510}$ Vgl. Mayring (2015), S. 55.

${ }^{511}$ Vgl. Wolf (2014), S. 152
} 
Größe, über die in der Studie eine Aussage getroffen werden soll“. ${ }^{512}$ Es wurden Kommentare von 1.707 Kunden gespeichert, von denen 461 als nicht relevant eingestuft wurden (zu den Selektionskriterien vgl. Anhang A). Insgesamt konnten 917 Kommentare bei „Skoobe“ und 328 Kommentare bei „Readfy“ ausgewertet werden, was einer Gesamtzahl von 1.245 Kommentaren entspricht. Die Festlegung des Kommentars als Analyseeinheit stellt sicher, dass die Aussage eines Kunden zu einem Merkmal nicht zweimal gewertet wird, was die angestrebte Häufigkeitsanalyse erlaubt.

Die Qualitätsmerkmale standen bereits vor der Materialdurchsicht fest. ${ }^{513}$ Die Merkmale (Kategorien) wurden also deduktiv im vorherigen Teil der Arbeit entwickelt. Die Festlegung von Kategorien im Vorfeld der empirischen Untersuchung trägt zur Vergleichbarkeit der Ergebnisse und damit auch zu ihrer Reliabilität bei. ${ }^{514}$ Des Weiteren wurden zur Erhöhung der Reliabilität - aber auch um gültige Daten zu erhalten - die Kategorien möglichst präzise definiert. Eine Kategorie ist das Ergebnis einer operationalen Definition, die sicherstellt, dass das Material in Daten überführt werden kann. Bei Bedarf wurde die Definition um Ankerbeispiele aus dem Material ergänzt und es wurden Codierregeln festgelegt. ${ }^{515}$ Dies ist nötig, weil bei vielen Kommentaren ein Interpretationsspielraum besteht, ob eine Codierung zulässig ist. ${ }^{516}$ Neben inhaltlichen wurden formale Merkmale codiert. Hierzu gehören Name des Appstores, Anbieter, Textumfang sowie Jahr und Monat der Veröffentlichung. Alle Aspekte sind im Codeplan ausführlich dargestellt (vgl. Anhang A). Für den ersten Materialdurchgang wurde eine Stichprobe von rund 500 Kommentaren gewählt (ca. $30 \%$ des Materials), da nicht jede Kategorie in jedem Kommentar enthalten ist. Es erfolgte eine Prüfung, ob das Material Auskunft zu den Merkmalen gibt und die Definitionen und Regeln sich als klar und eindeutig genug erweisen. Bei dieser Durchsicht des Materials konnten weitere Kategorien induktiv aufgedeckt werden, welche die im Vorfeld ermittelten Qualitätsmerkmale der Bereitstellung ergänzen. Dazu gehören bei der Dimension „Inhaltliche Vielfalt und Ordnung“ die Merkmale Bestandserweiterung und Kontinuität des Bestands sowie die inhaltliche Qualität der Bücher. Außerdem wurde beim Merkmal Übereinstimmung des Bestands mit Präferenzen deutlich, dass sich spezifische (inhaltsgebundene) Präferenzen als besonderer Faktor erweisen, weshalb das Merkmal in zwei Kategorien aufgeteilt wurde. Bei der Dimension „Kosten“ konnten noch die Merkmale Finanzierung (Kunden sind sich bewusst, dass das Angebot bzw. die Bücher finanziert werden müssen) und Niedrige Kosten als Trostpflaster (Kunden akzeptieren negative Aspekte eher, wenn die finanziellen Kosten gering sind) identifiziert werden. Beim räumlich-zeitlichen Zugang wurde deutlich, dass nicht nur die Option des Offline-Lesens relevant sein kann, sondern auch über welchen Zeitraum ohne Netzverbindung gelesen werden kann.

\footnotetext{
${ }^{512}$ Früh (2015), S. 91.

${ }^{513}$ Vgl. Früh (2015), S. 147.

514 Vgl. Mayring (2015), S. 52.

${ }^{515}$ Vgl. Mayring (2015), S. 97; vgl. Früh (2015), S. 123.

${ }^{516}$ Vgl. Früh (2015), S. 115 - 125.
} 
Bei der Technologie-Dimension zeigte sich, dass Kunden mitunter selbst Lösungen für Kompatibilitätsprobleme finden und beim Zugang mit mehreren Geräten gab es Hinweise, dass im Hinblick auf lesebezogene Daten die Möglichkeit der automatischen Synchronisierung von Geräten wichtig sein kann. Bei den Vergleichsprozessen konnten die Kategorien Vergleich zur Öffentlichen Bibliothek und Vergleich mit Kauf von Büchern im Handel bestimmt werden sowie die Kategorien Vergleich der technologischen Bedingungen (gedruckt/elektronisch), Lesertyp (Vergleich der eigenen Leseintensität mit anderen Personen) und Leserückkehr (Feststellung, dass man mit dem Angebot wieder (regelmäßig) Bücher liest).

\subsection{Maßnahmen zur Sicherstellung der Reliabilität der Ergebnisse}

Dem Aspekt der Reliabilität wurde besondere Aufmerksamkeit entgegengebracht, da Kunden häufig Alltagssprache verwenden und ihre Aussagen - im Vergleich zu journalistischen Inhalten - teilweise mehrdeutig oder missverständlich sind. Um zu prüfen, ob die Ergebnisse sich trotzdem reproduzieren lassen, wurde ein Reliabilitätstest durchgeführt. ${ }^{517}$ Zwei Codierer und die Bearbeiterin selbst haben 103 Kommentare ( $n=40$ bei Readfy; $n=63$ bei Skoobe) unabhängig voneinander codiert, um herauszufinden, ob sie in ihren Codierungen übereinstimmen (vgl. Tab. 7.1).

Tab. 7.1 Reliabilitätswerte für einzelne Kategorien, $\mathrm{C} 1=$ Codierer 1, C2 = Codierer 2, B = Bearbeiterin; für nicht dargestellte Kategorien liegen keine Messwerte vor; ausgewiesen wird das Reliabilitätsmaß nach Holsti, bei dem Werte immer paarweise verglichen werden; * Kategorie wurde später in zwei Kategorien aufgespalten

\begin{tabular}{|l|l|l|l|l|l|l|}
\hline Kategorienbezeichnung & $\begin{array}{l}\text { C1/ } \\
\text { C2 }\end{array}$ & Abw. & $\begin{array}{l}\text { C1/ } \\
\text { B }\end{array}$ & Abw. & $\begin{array}{l}\text { C2/ } \\
\text { B }\end{array}$ & Abw. \\
\hline Art der Analyseeinheit (Relevanzbestimmung) & .90 & 10 & .95 & 5 & .91 & 9 \\
\hline Offline-Lesen & .98 & 2 & .99 & 1 & .99 & 1 \\
\hline Geräteeigenschaften & .94 & 6 & .97 & 3 & .93 & 7 \\
\hline $\begin{array}{l}\text { Kompatibilitätsprobleme mit Gerätetypen und } \\
\ldots\end{array}$ & .95 & 5 & .97 & 3 & .97 & 3 \\
\hline Zugriff mit mehr als einem Gerät & .99 & 1 & .98 & 2 & .97 & 3 \\
\hline Bücher lesen & .83 & 17 & .83 & 17 & 1 & 0 \\
\hline Bücher suchen/ finden & .97 & 3 & .98 & 2 & .95 & 5 \\
\hline
\end{tabular}

${ }^{517}$ Vgl. Früh (2015), S. 117, S. 181. 


\begin{tabular}{|c|c|c|c|c|c|c|}
\hline Customization bei der Suche nach Büchern ... & .97 & 3 & .98 & 2 & .99 & 1 \\
\hline Personalisierung beim Lesen der Bücher & .99 & 1 & 1 & 0 & .99 & 1 \\
\hline Aktualität der Bücher im Bestand & .99 & 1 & .98 & 2 & .97 & 3 \\
\hline Bestandserweiterung & .99 & 1 & .99 & 1 & 1 & 0 \\
\hline Größe des Bestands & .87 & 13 & .91 & 9 & .96 & 4 \\
\hline Bekanntheit/ Popularität der Bücher im Bestand & .99 & 1 & .98 & 2 & .99 & 1 \\
\hline Inhaltliche Qualität der Bücher & .97 & 3 & .98 & 2 & .99 & 1 \\
\hline Vielfalt der Bucharten im Bestand & .95 & 5 & .94 & 6 & .97 & 3 \\
\hline Verfügbarkeit von Serien und Reihen & 1 & 0 & 1 & 0 & 1 & 0 \\
\hline $\begin{array}{l}\text { Übereinstimmung mit persönlichen Präferen- } \\
\text { zen* }\end{array}$ & .97 & 3 & .96 & 4 & .97 & 3 \\
\hline Finanzielle Kosten: keine Erwähnung & .85 & 15 & .86 & 14 & .85 & 15 \\
\hline $\begin{array}{l}\text { Sparpotenzial im Vergleich zur Angebotsform } \\
\text { Kauf }\end{array}$ & .98 & 2 & .97 & 3 & .99 & 1 \\
\hline $\begin{array}{l}\text { Sparpotenzial im Vergleich zur Angebotsform } \\
\text { Leihe }\end{array}$ & 1 & 0 & .99 & 1 & .99 & 1 \\
\hline Sparpotenzial: Andere & .94 & 6 & .95 & 5 & .99 & 1 \\
\hline $\begin{array}{l}\text { Keine/ Niedrige finanzielle Kosten als Trost- } \\
\text { pflaster }\end{array}$ & .99 & 1 & .98 & 2 & .97 & 3 \\
\hline Kosten-Buchlesemengen-Verhältnis & .98 & 2 & .97 & 3 & .97 & 3 \\
\hline Finanzierung & .97 & 3 & .97 & 3 & 1 & 0 \\
\hline Werbung stört beim Lesen von Büchern & .98 & 2 & .97 & 3 & .95 & 5 \\
\hline Werbung stört nicht beim Lesen von Büchern & .99 & 1 & .96 & 4 & .96 & 4 \\
\hline $\begin{array}{l}\text { Vorteile und Nachteile Technologie (elekt./ ge- } \\
\text { druckt) }\end{array}$ & .99 & 1 & .99 & 1 & 1 & c \\
\hline Vergleich mit öffentlichen Bibliotheken & .98 & 2 & .98 & 2 & 1 & 0 \\
\hline Vergleich mit Kauf von Büchern im Handel & .98 & 2 & .97 & 3 & .97 & 3 \\
\hline Lesertyp & .96 & 4 & .92 & 8 & .96 & 4 \\
\hline Freiheit bei Buchlesemenge & .94 & 6 & .96 & 4 & .96 & 4 \\
\hline Freiheit bei Lesezeitraum & .99 & 1 & .99 & 1 & 1 & 0 \\
\hline Freiheit bei Lesebeginn & .98 & 2 & .99 & 1 & .97 & 3 \\
\hline Freiheit Leseabbruch wegen Nichtgefallen & .99 & 1 & .99 & 1 & 1 & 0 \\
\hline
\end{tabular}




\begin{tabular}{|l|l|l|l|l|l|l|}
\hline $\begin{array}{l}\text { Entkopplung von finanziellen Kosten und Lek- } \\
\text { türe }\end{array}$ & .96 & 4 & $\underline{.99}$ & 1 & $\underline{.95}$ & 5 \\
\hline Leichtigkeit der Benutzung & .99 & 1 & 1 & 0 & .99 & 1 \\
\hline
\end{tabular}

Bei den grau hinterlegten Kategorien lassen sich die Abweichungen auf Missverständnisse und teilweise auf fehlende Sorgfalt zurückführen. Hier hätte eine intensivere Codierschulung im Vorfeld Abhilfe geschaffen. Auch erfordert der Codierprozess eine konstant hohe Konzentration. Die operationalen Definitionen sind jedoch passend und die Kategorien trennscharf, sodass keine Überarbeitung notwendig war. Dies war anders bei den unterstrichenen Werten in der Tabelle. Hier musste die Definition überarbeitet und weitere Codierregeln bestimmt werden, da sich die relevanten Aussagen der Kunden (gesuchter Bedeutungsgehalt) zunächst nicht eindeutig finden und interpretieren ließen, was jedoch unerlässlich ist, um valide Ergebnisse zu erhalten. Bis auf „Kontinuität des Bestands“, „Fremdsprachige Bücher im Bestand“ und „Selbstdisziplinierung durch finanzielle Ausgaben“ konnten alle Merkmale der Bereitstellungsqualität geprüft werden; ebenso alle Merkmale der Vergleichsprozesse bis auf „Leserückkehr“. Bei den Aktivitäten und beim Optimierungspotenzial waren deutlich mehr Merkmale nicht in der Teststichprobe enthalten. Allerdings ist die Wahrscheinlichkeit hoch, dass sie in der gesamten Stichprobe nicht erwähnt werden. Die Definitionen wurden jedoch von beiden Codierern als eindeutig und verständlich bewertet, sodass insgesamt von einer akzeptablen Reliabilität der Ergebnisse auszugehen ist.

\subsection{Darstellung der Ergebnisse}

1. Frage: Erwähnen Kunden die theoretisch abgeleiteten Qualitätsmerkmale der Bereitstellung von Büchern? Welche Merkmale werden am häufigsten genannt?

Alle als potenziell relevant eingestuften Qualitätsmerkmale wurden von Kunden erwähnt bis auf die Strategie der Selbstdisziplinierung durch finanzielle Ausgaben (vgl. Tab. 7.2).

Tab. 7.2 Übersicht der Qualitätsmerkmale bei der Bereitstellung von Büchern (Quelle: Eigene Erstellung)

\begin{tabular}{lllll}
$\begin{array}{l}\text { Inhaltliche Viel- } \\
\text { falt } \\
\text { \& Ordnung }\end{array}$ & $\begin{array}{l}\text { Zeit \& } \\
\text { Raum }\end{array}$ & Basistechnologie & Kosten & $\begin{array}{l}\text { Leichtigkeit } \\
\text { der } \\
\text { Benutzung }\end{array}$ \\
\hline - Strukturierung & - Freiheit & - Kompatibilitätsproble- & - Freiheit bei der Buch- & - Leichtigkeit \\
des & beim & me $\checkmark$ & lesemenge $\checkmark$ & der \\
Buchbestands $\checkmark$ & Lesebeginn & & & Benutzung \\
& $\checkmark$ & & $\checkmark$ \\
\hline
\end{tabular}




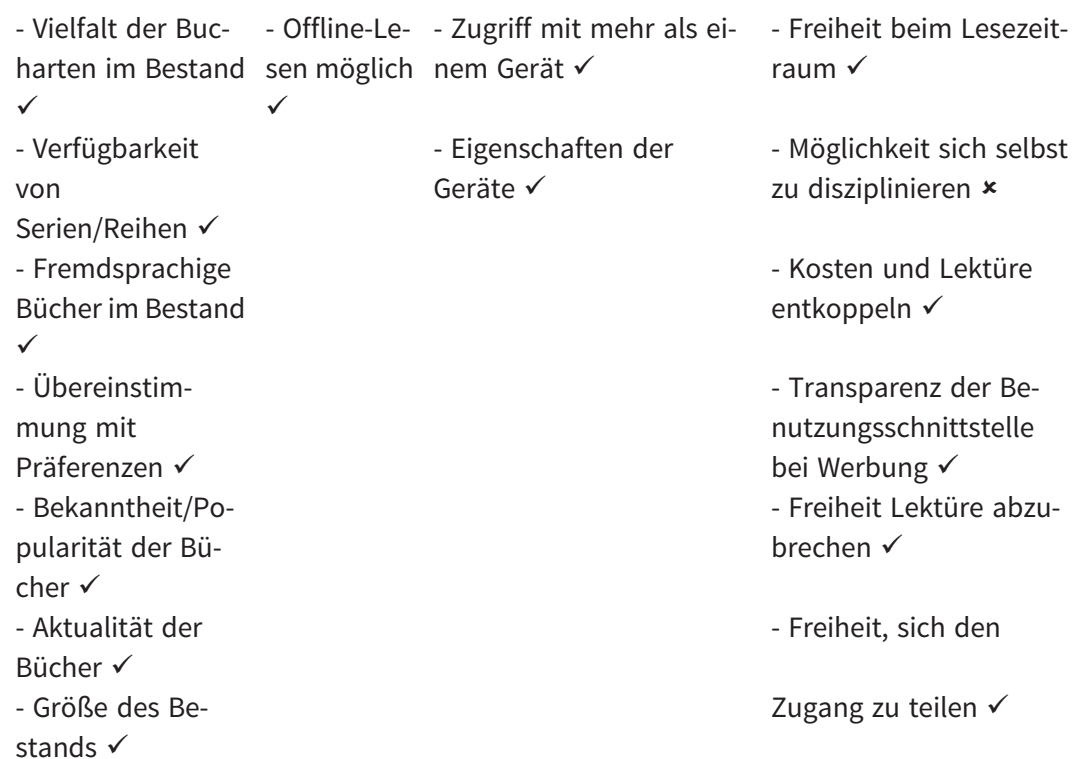

Mögliche Erklärungen für diesen Befund können sein, dass Kunden von Skoobe finanzielle Ausgaben als Mittel der Selbstkontrolle unbewusst anwenden und daher nicht erwähnen oder das Angebot wird vorwiegend von Personen genutzt, die bereits regelmäßig lesen.

Bereitstellungsdimension: Basistechnologie

Bei den Kompatibilitätsproblemen haben 8 Kunden eigene Lösungen gefunden, um das Angebot trotzdem nutzen zu können (z. B. Wechsel auf ein ungewohntes Lesegerät). 22 Kunden haben die Geräteeigenschaften negativ bewertet (vgl. Abb. 7.2).

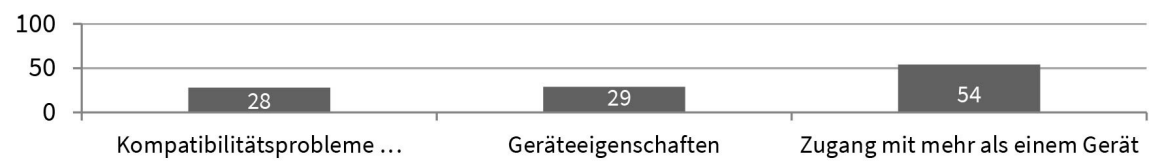

Abb. 7.2 Merkmale der Bereitstellungsdimension „Basistechnologie“, Anzahl der Kunden

Das Merkmal, dass ein Zugriff mit mehreren Geräten erlaubt ist, wird für Kunden auch dann wichtig, wenn sie sich den Zugang mit anderen Personen teilen. Den 
Äußerungen von 14 Personen (nur Skoobe-Kunden) lässt sich entnehmen, dass sie dies tun. ${ }^{518}$ Zum erwähnten Personenkreis gehören nur Familienmitglieder.

„Bei uns liest die ganze Familie auf unseren Tablets. “ (443)

„Meine Kinder leihen sich meinen Kindle immer öfter und zwar nicht zum spielen sondern zum Lesen!!! Toll!“ (458)

„Meine Frau und ich sind beide Leseratten. Ein Leben ohne Skoobe ist derweil undenkbar!“ (1346)

„Uns gefällt es Wir haben skoobe inzwischen auf drei tablet installiert und lieben es alle. “(1646)

Bereitstellungsdimension: Inhaltliche Vielfalt und Ordnung der Bücher

Der Tabelle lässt sich die Häufigkeitsverteilung der Qualitätsmerkmale entnehmen (vgl. Tab. 7.3).

Tab. 7.3 Merkmale der Bereitstellungsdimension „Inhaltliche Vielfalt \& Ordnung der Bücher“, Anzahl der Kunden

\begin{tabular}{|l|l|}
\hline Größe des Bestands & 467 \\
\hline Aktualität der Bücher & 87 \\
\hline Vielfalt der Bucharten & 70 \\
\hline Erweiterung des Bestands & 64 \\
\hline Bekanntheit/ Popularität der Bücher & 52 \\
\hline Strukturierung des Buchbestands & 27 \\
\hline Fremdsprachige Bücher & 13 \\
\hline Inhaltliche Qualität der Bücher & 11 \\
\hline Vollständigkeit von Serien und Reihen & 10 \\
\hline Kontinuität des Bestands & 6 \\
\hline
\end{tabular}

Im Hinblick auf die Beurteilung des Bestands zeigt sich, dass Kunden eine „Verbesserung“ des Titelbestands im Zeitverlauf erwarten. Bei 64 Personen ließ sich die Anspruchshaltung nachweisen. Sechs Personen schildern negative Erlebnisse, bei denen ein Titel, den sie lesen wollten oder gerade gelesen haben, nicht mehr im Bestand verfügbar war. Die Ursache hierfür ist vermutlich, dass der Anbieter zeitlich limitiert Lizenzrechte erwirbt und diese nach Ablauf der Gültigkeit vom Verlag nicht

${ }^{518}$ Es besteht die Annahme, dass Kunden sich den Zugang teilen, da zwei Benutzerkonten doppelte Kosten verursachen. Auch in Bibliotheken teilen sich Familien einen Ausweis, um Geld zu sparen (Vgl. 4.5.1 Finanzielle Kosten). 
verlängert wurden. 20 von 27 Personen bewerten zudem die Strukturierung des Bestands als positiv:

„man kann wunderbar wählen, durch eingeteilte Genres und jene ist wieder in „meistgelesen/ neue Bücher etc" unterteilt. Also toll aufgegliedert, was die Siche nach einem guten Roman/ Sachbuch etc vereinfacht" (454)

„Gut, auch Amazon hat jetzt einen Leihdienst, aber solange da nicht irgendein System dahinter steht und man von der ungeheuren Anzahl der Bücher regelrecht erschlagen wird, ist Skoobe die bessere Alternative" (430)

In 295 Aussagen thematisieren Kunden die Frage, ob sie Bücher finden, die sie lesen möchten. Aus der Tatsache, dass Kunden mit spezifischen Präferenzen den Bestand häufiger negativ bewerten (vgl. Abb. 7.3), kann geschlussfolgert werden, dass die Gefahr der Enttäuschung bei inhaltsgebundenen Präferenzen größer ist als bei Personen, die bereit sind, sich auf ähnliche Optionen einlassen. Tatsächlich nimmt ein Großteil der Kunden aus der Stichprobe einige Enttäuschungen in Kauf, solange sie ausreichend viele andere Bücher finden, die sie mögen. Dies zeigt sich an der überwiegend positiven Gesamteinschätzung der beiden Bestände im Hinblick auf die Übereinstimmung mit persönlichen Präferenzen (vgl. Abb. 7.3).
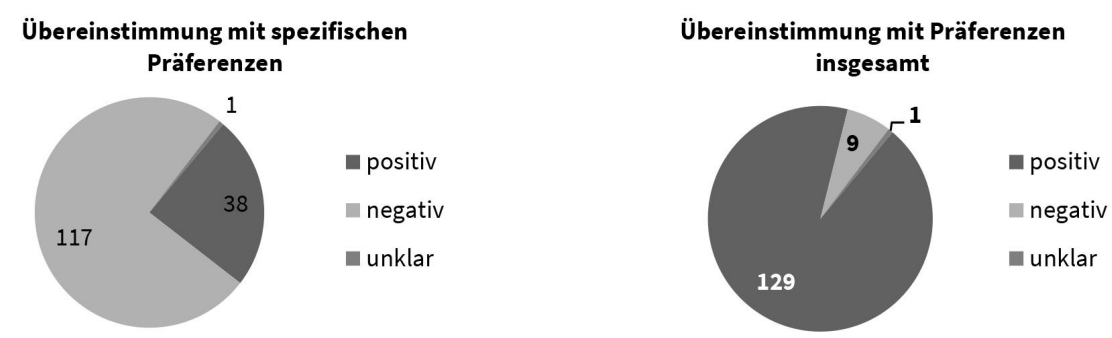

Abb. 7.3 Wahrgenommene Übereinstimmung des Bestands mit persönlichen Präferenzen, Anzahl der Kunden

1.1 Unterfrage: Sind „Verfügbarkeit gesuchter Bücher“ und „hohes Anspruchsniveau" hemmende Faktoren in Bezug auf die Übereinstimmung des Buchbestands mit Kundenpräferenzen?

Bei der Untersuchung bestätigt sich, dass Kunden in einer Suchsituation mit konkreten Vorgaben (Autor, Titel) eher enttäuscht werden. Dieser Umstand ist darauf zurückzuführen, dass der Buchbestand der Mietangebote weniger Buchtitel umfasst als im Buchhandel lieferbar sind.

„Eigentlich ok, aber. .. Leider fehlen mir hier ein paar Autoren aus dem Psychothriller-Genre. Z. B. Michael

Robotham und Karen Slaughter" (216) 
„schade ist, ist das es hier leider nicht von allen Schriftstellern Bücher gibt.wie z.b. ,Olsen oder nesbø" (520)

Auch die Suche nach Novitäten führt dazu, dass Kunden mit dieser Vorgabe häufig nicht fündig werden. Diese Situation entsteht dadurch, weil Verlage überwiegend Lizenzen für Buchtitel aus ihrer Backlist, also für ältere Bücher vergeben. ${ }^{519}$ Insgesamt werden fehlende Sucherfolge bei Novitäten als weitestgehend unproblematisch wahrgenommen.

„Die neuesten Bücher sind zwar nicht vorhanden aber sehr, sehr viele sehr gute Bücher" (307)

„Die allerneuesten Bestseller sind nicht verfügbar aber es gibt ja auch tolle Bücher die nicht erst gestern erschienen sind." (458)

Auch kommt es vor, dass Kunden bekannte Angebotsformen für den temporären Erwerb heranziehen, um besser einschätzen zu können, was realistisch bestimmte Erwartungshaltungen sind.

„Ok, keine Neuerscheinungen, aber das hatte ich auch nicht erwartet. Filme kommen ja auch erst ins Kino bevor sie in die Videothek kommen" (571)

„Wie in jeder Bücherei sind auch hier nicht alle Titel erhältich, aber dann wartet man oder besorgt sie sich doch wieder gebraucht." (474)

Nur bei 9 Personen lag der Fall vor, dass kein einziges Buch oder nur wenige Titel die Eliminationsphase überstanden haben, also dem Anspruchsniveau genügten.

„Habe stundenlang in sämtlichen Kategorien gestöbert, hunderte von Beschreibungen gelesen und mich trotzdem nie so richtig angesprochen gefühlt" (71)

"allerdings finde ich nur wenige Bücher, die meinen Geschmack treffen" (412)

"habe nichts im Angebot gefunden, was mich interessiert hat" (665)

Bei diesen Beispielen geht es also nicht darum, dass konkrete Titel oder Themen nicht gefunden wurden. Eine solche Situation wird von Kunden allerdings auch beschrieben. Gleichzeitig werden teilweise die negativen Emotionen, die damit einhergehen, deutlich:

„Alle Bücher die ich will gibt es irgendwie nicht! Das nervt!“ (852)

„Geht garnicht Keiner meiner Lieblingsautoren, Bücher zu finden“ (120)

„leider gab es zu speziellen Themen sehr wenig Auswahl“ (538)

„Leider habe ich von 20 gesuchten büchern nur 2 gefunden, ist also eine schlechte quote. Für mich keine alternative zur onleihe verschiedener bibliotheken, die schon für 10 euro JAHRESBEITRAG zu haben ist" (750)

${ }^{519}$ Vgl. Lenz (2014)/ Buchreport, S. 65; vgl. Kornmeier (2016). 


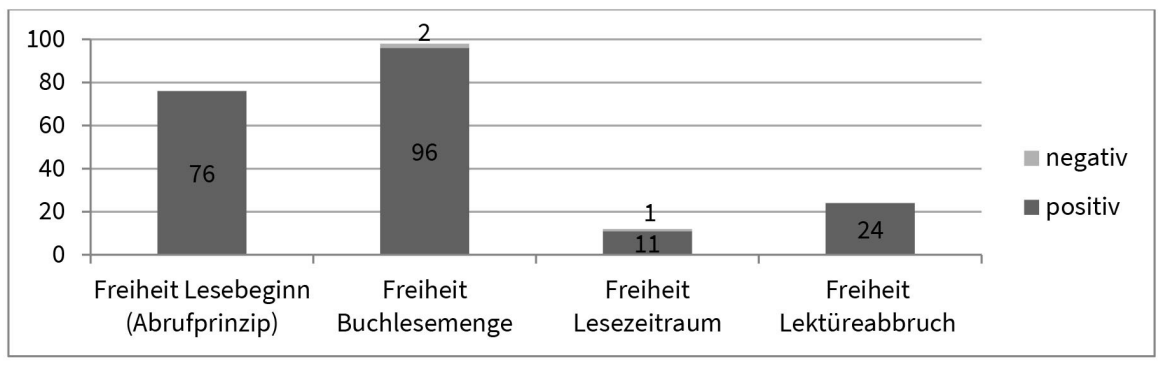

Abb. 7.4 Merkmale der wahrgenommenen Freiheit beim Bücherlesen, Anzahl der Kunden

Wahrgenommene Freiheit beim Bücherlesen

Im Hinblick auf die wahrgenommene Freiheit bei der Lektüre sind verschiedene Dimensionen der Bereitstellungsqualität betroffen (vgl. Abb. 7.4).

Die Freiheit beim Lesebeginn ist gegeben, wenn Kunden den Lesezeitpunkt und den -ort selbst bestimmen und ihre Lesebedürfnisse sofort befriedigen können (vgl. Abb. 7.4). Sie hängt einerseits von der Ubiquität $a b$ und andererseits von Beschränkungen, die ein Anbieter vornimmt.

Beim Abrufprinzip schätzen Kunden es, dass sie sich die Wegekosten sparen.

„man kann bei so vielen Gelegenheiten noch ein paar seiten lesen. Handy oder Tab hat man fast immer dabei“" (874)

„So stelle ich mir zeitgemäßes Lesen vor. Keine Laufereien zu Buchhandlungen und Bibliotheken mehr" (914)

"Nie mehr Langeweile im Wartezimmer im bus oder sonstwo" (1353)

„Für mich ganz wichtig, keine Wartezeiten um ein Buch ausleihen zu können“ (1017)

In Bezug auf den selbstbestimmten Lesebeginn spielt auch die Offline-Lesen-Option eine Rolle, da diese bei fehlender Netzverbindung die Ubiquität sicherstellt. 69 Personen haben die Option erwähnt. 11 davon ist die Dauer der Offline-Nutzung wichtig. 3 Personen ist die Option egal.

„Einen Stern ziehe ich ab, weil ich im Basic-Tarif alle 24 Stunden Online gehen muss um die „eigenen“ Bücher zu lesen. Das habe ich letztens vor dem Rückflug vergessen und konnte nicht eine einzige Seite im Flugzeug lesen :-( Es sollte wenigstens ein Offline-Buch für den Notfall geben" (1234)

Bei beiden Angeboten gibt es keine Beschränkung der absoluten Büchermenge, die gelesen werden kann, was Kunden wertvoll finden (vgl. Abb. 7.4): 
Des Weiteren wird bei den Angeboten (bei Readfy nur in der Werbeversion) die absolute Lesedauer nicht eingeschränkt (vgl. Abb. 7.4):

„und hat dann Zeitdruck beim lesen.Ich bin kein Freund davon ein Buch über mehrere Wochen zu lesen, aber ich möchte das Gefühl haben ich könnte,wenn ich wollte. Lesen unter Zeitdruck gefällt mir nicht." (459)

24 Kunden schätzen es, dass ihnen keine Folgekosten durch Fehlentscheidungen bei der Buchauswahl entstehen (vgl. Abb. 7.4) ${ }^{520}$ :

„Ist genau das Richtige für Leute, die sich ärgern Bücher zu kaufen, die dann doch nichts sind im Nachhinein." (1048)

Die wahrgenommene Freiheit beim Bücherlesen wird bei einigen Kunden auch dadurch gefördert, dass sie nicht über die Kosten nachdenken müssen. Sie können unbeschwerter lesen, weil sie nicht das Gefühl haben, ihren Buchkonsum einschränken zu müssen. 51 Personen (45 davon Skoobe-Kunden) erwähnen die Entkopplung von finanziellen Kosten und Lektüre. Dies kann als erstes Indiz gewertet werden, dass im Buchbereich bei einigen Kunden eine Flatrate-Präferenz besteht, jedoch (bei Viellesern) kein Flatrate-Bias:

Da ich sehr viel lese wurde es so langsam ein teures Hobby (...) Jetzt kann ich endlich entspannt lesen ohne die Haushaltskasse zu sehr zu strapazieren (461)

\section{Bereitstellungsdimension: Kosten/Opfer}

Für 24 Personen (23 davon Readfy-Kunden) sind niedrige/keine Kosten ein Trost dafür, dass sie ihre Erwartungshaltung anpassen müssen.

„Ist klar das es nicht alle Bücher gibt...die App ist ja auch kostenlos“ (175)

Die Dominanz der Readfy-Kunden in diesem Punkt erklärt sich überwiegend dadurch, dass sie Werbeeinblendungen beim Bücherlesen als belastend empfinden.

„Geschenkten Gaul, schaut man nicht ins Maul (...). Werbung okay muss sein“ (78)

Hier kann nicht abgeschätzt werden, wie lange der Zustand der Akzeptanz andauert. Allgemein wird das Anspruchsniveau bei bestimmten Merkmalen wie Transparenz der Benutzungsschnittstelle und Buchbestand v. a. bei kostenlosen Angeboten (zeitweise) gesenkt und negative Emotionen toleriert, weil dem Kunden keine oder nur geringe finanzielle Kosten entstehen. ${ }^{521} 24$ Personen erwähnen das Thema Fi-

\footnotetext{
${ }^{520}$ Vgl. auch Skoobe (2014).

521 Vgl. auch Wolf (2014), S. 190.
} 
nanzierung, allerdings nur Kunden von Readfy, also einem kostenlosen Angebot. Die Äußerungen stehen fast alle im Zusammenhang mit dem Thema Werbung.

Super Dezente Werbebanner (...) So unterstütze ich die Autoren gerne. Die Mondpreise für eBookKäufe haben mich zur Piraterie getrieben, diese App hat mich wieder zurück geholt (290)

Kunden versuchen sich die Bedeutung der Werbung klar zu machen und sich in ihrer Akzeptanzhaltung zu bestärken. Dies klappt nicht immer:

die werbung lässt mich bald explodieren! !! (...) Ich verstehe euer Prinzip und alles aber das geht nicht sorry!! (35)

1.2 Unterfrage: Wird Werbung als belastende Störung empfunden? Hängt das Ausmaß der wahrgenommenen Störung vom Werbemittel ab (reizarm/reizstark)? Gibt es Hinweise darauf, dass Kunden Werbevermeidungsstrategien anwenden?

126 Aussagen thematisieren die Transparenz der Benutzungsschnittstelle bei Werbeeinblendungen. In 77 Aussagen wird Werbung als eine belastende Störung beim Lesen dargestellt, während 49 Aussagen dieses Empfinden nicht ausdrücken. Reizstarke Werbung wird häufiger thematisiert, wenn Werbung als negativ bewertet wird (16 Personen gegenüber 2 Personen). Dabei nehmen Kunden Bannerwerbung als weniger belastende Störung wahr. Die Unterbrechung der Transparenz hemmt den Lesefluss in diesem Fall eher nicht:

„die Werbung nervt. Die App funktioniert wunderbar aber ich würde auch ein paar Euro für eine kostenpflichtige

App ausgeben wenn ich mir dafür das Werbegeblinke am unteren Bildrand ersparen könnte. Oder wenn schon Werbung dann nur statische" (12)

„Einzige Einschränkung des Vergnügens ist die Werbung. In Kopf- und Fußzeile geht's, aber die ganzseitigen Einblendungen nerven." (143)

Als besonders belastend werden hingegen animierte Werbeformen und Pop-UpWerbung erlebt. Insbesondere die Lesesituation, bei der ein sofortiger Eingriff (Fenster schließen) technisch unterbunden wird, wirkt sich negativ auf die Akzeptanzhaltung aus.

„Mit der Werbung kann ich soweit auch leben, nur die blinkenden Banner sind sehr sehr anstrengend, besonders wenn man abends im Bett noch $n$ bisschen liest. “ (45)

„Werbung weiter deutlich ausgebaut Früher kam Werbung und man konnte sie einfach weg klicken. Jetzt erscheint Werbung über eine ganze Seite, die man nicht mehr weg klicken kann und nach einiger Zeit von selbst wieder verschwindet. Da ist für mich die Grenze überschritten. Schade! Es geht aber immer noch schlechter: neuerdings (19.03.16)erscheinen sogar Werbefilme, die man sich einige Sek. ansehen muss. Erst dann kann man ihn wegdrücken. "(57)

Die negative Bewertung eines reizstarken Werbemittels kann aber auch durch das Umfeld des Kunden bedingt sein, wenn es zu einem für den Kunden unangenehmen Erlebnis kommt: 
„Die Werbung stört an sich nicht. Nur spät Abends sollte auf die Wiedergabe von Sounds oder Videos verzichtet werden. Nichts ist schlimmer als plötzlicher Krach aus dem Handy. Für Leute die gerne spät lesen und nicht allein leben kann das schon manchmal kritisch werden" (334)

4 Kunden erwähnen starkes Involvement als Strategie, um äußere Reize abzuwehren.

„Wenn ich so richtig in der „Scheinwelt" versunken bin, ist, bei Werbung, das drücken auf den "Weiterknopf" automatisch. Dann stört auch Sie nicht mehr so sehr." (93)

„Die blinkende Werbung die hier öfter zur Sprache kommt stört mich ganz und gar nicht - beim lesen setzt mein

Kopfkino ein und dann stört auch sowas nicht mehr" (55)

2 Kunden davon machen deutlich, dass es auch vom Inhalt des Buches abhängt, wie gut ihnen das Ausblenden von externen Reizen gelingt.

„und das mit der Werbung... Wenn das Buch fesselt, sieht man die Werbung garnicht" (61)

„Die Werbung ist gewöhnungsbedürftig (...) Muss auch dazu sagen wenn man ein gutes Buch liest achtet man da eh nicht mehr so drauf" (98)

Hieraus lässt sich die Annahme ableiten, dass Leser mit Werbung im Buch weniger gut zurechtkommen, wenn ihnen der emotionale oder kognitive Zugang zum Text schwerfällt oder wenn Lernergebnisse das Ziel des Leseprozesses sind (schulischer/ beruflicher Kontext).

2. Frage: Welchen buchbezogenen Aktivitäten gehen Kunden in den Angeboten nach? Welche Aktivitäten werden am häufigsten genannt?

Wie bereits bei der Analyse der Kundenaktivitäten im vorherigen Teil der Arbeit deutlich wurde, bedingen sich die Aktivitäten Bücher lesen, Bücher auswählen und Bücher organisieren (insbesondere Merklisten anlegen) gegenseitig. Sie sind funktional miteinander verbunden oder der Input aus der einen wird bei einer anderen Aktivität verwendet. Im Alltag sind sie die vermutlich am häufigsten ausgeübten buchbezogenen Aktivitäten (vgl. Kap. 6.1). Dies kann eine Erklärung dafür sein, warum sie auch am häufigsten thematisiert wurden. Alle anderen Aktivitäten, nach denen im Material gesucht wurde, fanden fast keine Erwähnung (vgl. Tab. 7.4).

Tab. 7.4 Übersicht der buchbezogenen Kundenaktivitäten, Anzahl der Kunden

\begin{tabular}{|l|l|}
\hline Bücher lesen & 491 \\
\hline Bücher suchen/stöbern & 240 \\
\hline Bücher organisieren & 35 \\
\hline Bücherlisten anderer Kunden ansehen & 3 \\
\hline Rezensionen schreiben bzw. lesen & 1 \\
\hline
\end{tabular}




\begin{tabular}{|l|l|} 
Bücher auf Skala bewerten bzw. Bewertungen ansehen & 2 \\
\hline Buch-/Textlinks mit anderen teilen & 1 \\
\hline Informationen zu Neuerscheinungen suchen/erhalten & 4 \\
\hline
\end{tabular}

Eine Erklärung dafür, warum andere Aktivitäten nicht/kaum genannt werden, könnte sein, dass sie gar nicht ausgeübt werden (vgl. 6.6.3) oder sie Handlungen einer Tätigkeit sind und daher nicht explizit erwähnt werden, z. B. „Rezensionen lesen“ und „Bewertungen ansehen“ als Bestandteile der Buchsuche. Zudem bewertet der Kunde, auf den die zwei Nennungen mit 1 zurückgehen, nur den Umstand, dass er die Möglichkeit hätte, Textlinks zu teilen und Bücher zu rezensieren, und nicht das Erleben bei der Aktivität: „Und mann kann das gelesen Rezensieren und Sterne vergeben, sowie auch in sozialen Netzwerken teilen“ (454). Hieraus lässt sich folgern, dass sich Kunden - wie von der CDL angenommen - in ihrem Urteil auf Ereignisse und Erlebnisse bei der Angebotsnutzung stützen, d. h. der Wert für den Kunden entsteht nicht dadurch, dass Funktionen vorhanden sind, sondern erst bei ihrer Nutzung. Werden Funktionen nicht verwendet bzw. Aktivitäten nicht ausgeübt, spielen sie für die Bewertung des Angebots keine Rolle. 2 von 3 Kunden, die Bücherlisten anderer Kunden erwähnen, beziehen sich z. B. auf Erlebnisse beim Stöbern in Leselisten anderer Kunden und beschreiben, welchen Vorteil diese Aktivität hat:

„Leselisten anderer User finde ich spitze zum Entdecken!“ (286)

„Interessant sind auch die Leselisten, die auf weitere Titel aufmerksam machen. " (345)

Ähnlich verhält es sich mit der Funktion „Informationen über neue Bücher erhalten“ als eine Form der Personalisierung. Sie zeichnet sich dadurch aus, dass die Aktivität, Neuigkeiten in Erfahrung bringen, nicht vom Kunden erbracht werden muss. Als Vorteil nehmen Kunden wahr, dass sie sich ohne Mühe ausreichend informiert fühlen. Wie jede Form der aneignenden Aktivität führt auch die Information über Neuigkeiten zu einem Erleben von Orientierung in der Welt:

„Bin zufrieden, hab alle Bücher immer dabei und verpasse keine Neuerscheinungen. “ (1598)

„Die Benachrichtigung über neue Bücher ist auch super. “ (1366)

".Man ist bestens informiert und wenn man es regelmaessig verfolgt ist man am neuesten Stand des ,Buechergeschehens'." (682)

„Mir gefällt auch gut, dass ein Überblick über die Neuerscheinungen gegeben wird.“ (449)

Somit führen erst die Wahrnehmung von Vor- und Nachteilen und das Erleben von Emotionen dazu, dass Aktivitäten bzw. Funktionen in die Urteilsbildung einfließen.

3. Frage: Besteht Optimierungspotenzial bei den Kundenaktivitäten? 


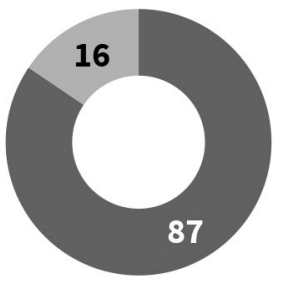

Optimierungspotenzial durch Customization

Optimierungspotenzial durch Personalisierung

Abb. 7.5 Optimierungspotenziale von Aktivitäten aus Kundensicht

\section{Customization: Suche (13 Personen)}

Kunden nennen hier Filter- und Sortierungsmöglichkeiten, um das Scannen des Angebots zu erleichtern bzw. den Bestand auf eine persönlich relevante Menge zu reduzieren. Ein Kunde wünscht sich die Möglichkeit, den Schriftgrad der Suchergebnisse vergrößern zu können (466) und andere Kunden die Option, eigene Merklisten alphabetisch sortieren zu können (z. B. 1020).

„Wünschenswert wäre auch eine komfortablere Suche, wie z. B. in dem sich die Ergebnisse sortieren lassen z. B. nach der Nummer der Ausgabe bei Serien oder Erscheinungsdatum" (73) „ich würde mir wünschen Kategorien irgendwie ausblenden zu können da ich bei der suche ständig Erotik angezeigt bekomme und ich finde es eher belästigend" (339)

\section{Customization: Organisation (7 Personen)}

Kunden sehen es als Vorteil, verschiedene Listen erstellen und Lesezeichen setzen zu können, erwarten aber auch, damit sehr flexibel gemäß den eigenen Bedürfnissen umgehen zu können:

„gut finde ich die Leseliste und Lesezeichen Funktion“ (212)

„Der Komfort könnte besser sein (z. B. Verschieben von Büchern zwischen den Leselisten)“ (119)

„Schön wäre es wenn man Bücher die man nur vielleicht lesen will, auf eine Liste vermerken könnte ohne sie erst runterzuladen.(...).Ebenso wenn man gelesene Bücher,die man nicht löschen möchte, gesondert ablegen könnte." (249)

„Schade dass man keine Lieblingsautoren sondern nur Buchtitel in die Merkliste eintragen kann“ (1011)

\section{Customization: Lesen (60 Personen)}

Einige Kunden bewerten, wie viele Einstellungsmöglichkeiten es insgesamt gibt.

„Schade das man sich die App nicht individuelle einstellen kann. Z.b. Schrift Farben Hintergrund. " (1307)

„nur leider bietet der integrierte Reader zu wenig Einstellungsmöglichkeiten“ (1655)

Im Einzelnen thematisieren Kunden die Lesezeichenfunktion, die Notizfunktion, einen Nachtmodus (Schwarzer Hintergrund bei Lesen ohne externe Lichtquelle) 
und die Änderung der Schriftgröße. Bei einigen Aussagen wird deutlich, warum eine Funktion wichtig ist z. B. um sich den Inhalt besser zu merken (Textstellen markieren) (1073) oder um Vokabeln nachzuschlagen, weil man z. B. in einer anderen Sprache liest (Kopierfunktion und/oder Direktzugriff auf Nachschlagewerk) (388, 1287). Darüber hinaus lässt sich eine eher kosmetische Form der Customization (Ausdruck persönlicher Vorlieben z. B. Änderung Schriftgrad (208), Zeilenabstand vergrößern (1269), Seitenfarbe ändern (630)) von einer instrumentellen Customization abgrenzen. Bei der zuletzt genannten Form sind Funktionen die Voraussetzung, um Lesestrategien (z. B. Textstellen markieren zur Verstehenssicherung) oder Suchstrategien (z. B. Scanning mittels automatischer Textsuche: Enthält das Buch relevante Textstellen? (1076)) anwenden zu können. Teilweise sorgt instrumentelle Customization auf operationaler Ebene überhaupt erst für die Möglichkeit einer Bedeutungskonstruktion (Zoomfunktion bei Abbildungen, Tabellen etc.):

„was mich aber stört: Bilder lassen sich dicht vergrößern und sind meist nicht erkennbar von der Größe her. Das verdirbt bei manchem Büchern den Lesespaß extrem. " (1064)

\section{Personalisierung: Suche (5 Personen)}

Hier erwähnen Kunden die automatische Anpassung an Präferenzen oder Ausblendung von uninteressanten Bucharten sowie automatische Buchvorschläge zu ähnlichen Büchern.

„Das einzige, was ich mir noch wünschen würde, wären persönliche Vorschläge, welche sich $a b$ den angegebenen Bewertungen orientieren. " (1431)

\section{Personalisierung: Organisation (7 Personen)}

Genannt werden die Anzeige, welche Bücher bereits gelesen wurde, und die Anzeige eines persönlichen Bereichs mit gerade/zuletzt gelesenen Büchern direkt nach dem Öffnen der App.

„Schön wäre außerdem noch, wenn auf der Hauptseite die letzten paar gelesen Bücher von einem selbst aufgelistet werden würden. Es sind einfach zu viele Klicks bis zu seiner Leseliste nötig." (102)

„Bloß schade, dass beim Öffnen der App nicht gleich die persönliche Bibliothek oder das zuletzt gelesene Buch mitgeöffnet wird" (166)

68

„Bisher musste ich mir immer alle Bücher aufschreiben (habe einen ganzen Leitzordner), da ich sehr viele Serien lese und sonst total den Überblick verliere. Aber bei Skoobe kann ich jederzeit sehen, welche Bücher ich bereits gelesen habe." (464) 


\section{Personalisierung: Lesen (3 Personen)}

Als vorteilhaft wird wahrgenommen, wenn automatisch die letzte Textstelle angezeigt wird.

„Was mir fehlt um richtig glücklich zu sein, ist die Möglichkeit direkt dort weiter zu lesen, wo ich beim letzten Mal aufgehört habe. Bis jetzt habe ich das nur durch das manuelle Setzen eines Lesezeichens hin bekommen. Was ich persönlich aber zu umständlich finde“ (265)

Von den 54 Personen, die Bücher auf mehreren Geräten lesen, haben 17 Personen eine Synchronisierungsfunktion erwähnt (alle als wichtig bewertet und/oder im Alltag genutzt)

„Gut fände ich, da ich das Buch auf verschiedenen Medien lese (smartphone \& ipad), wenn ich jeweils auf derselben Seitenzahl wäre" (27)

4. Frage Lassen sich bei der Urteilsbildung des Kunden Vergleichsprozesse nachweisen? Wenn ja, lassen sich konkret Vergleiche im Hinblick auf Sparpotenzial bei einem Angebot und das KostenBuchlesemengen-Verhältnis feststellen?

45 Personen haben sich mit den Vor- und Nachteilen der Basistechnologie auseinandergesetzt, also wie sie den Umgang mit E-Books im Alltag im Vergleich mit gedruckten Büchern erleben. 273 Personen setzen sich mit der eigenen Leseridentität auseinander, indem sie sich einem Lesertyp zuordnen. Der Großteil der Personen sieht sich selbst als Vielleser. Diese klare Zuordnung legt die Vermutung nahe, dass Kunden ihre Nutzungsmenge eher nicht überschätzen, sondern über ein ausgeprägtes Wissen in Bezug auf ihr eigenes Leserverhalten verfügen. Ferner vergleichen Kunden, was sie bei der Angebotsnutzung erleben bzw. wie sie sich verhalten mit der Art und Weise, wie sie sich bei anderen Angebotsformen verhalten bzw. dort erleben.

\section{Vergleich: Kauf im Handel (30 Personen)}

„ich habe hier Bücher gefunden die ich noch nie im Handel gesehen habe“ (1576)

„die buecher is fantastisch hab bereits mehrere buecher hier gefunden die ich im laden nie anruehren wuerde" (722)

„Toll finde ich auch, dass ein Buch, dass gar nicht gefällt, einfach ausgetauscht werden kann. Hätte ich es kaufen müssen, wäre es teurer geworden" (529)

„Gerade wenn man mal n neues Genre ausprobieren will ohne sich gleich die Bücher, für zum Teil viel Geld, kaufen will" (1139)

„Ich lese Bücher einmal, dann nie wieder. Also optimal für mich“ (1381)

„Wenn ich meine Lesephasen habe (3 - 7 TB/Woche), kostete das (...) viel Arbeit..denn nicht jedes Buch lese ich zweimal und habe sie danach wieder verkauft. " (474)

„Ich wollte gerade das buch das ich am lesen war weiter lesen und hab fast einen anfall bekommen. Es war einfach nicht mehr da. Ich hatte es bis zur Hälfte durch und dann das. Ich bin so sauer.app wird jetzt sofort gelöscht und das buch einfach bei Amazon bestellt." (79) 
In den Aussagen zeigt sich, dass Kunden auf andere Weise durch den Bestand navigieren als im Laden und Bucharten für sich entdecken, die sie dort nicht wahrnehmen. Ein Grund könnte, dass sie wegen der attributorientierten Anordnung (Warengruppe bzw. Buchart) nur bei bestimmten Kategorien/in bestimmten Abteilungen verweilen oder weil bestimmte Bucharten und Buchtitel nicht im Laden präsentiert werden. Auch betonen Kunden die geringen Reversibilitätskosten, was ebenfalls dazu führen kann, dass neue Bucharten angelesen werden, sowie den Vorteil des temporären Erwerbs beim Einmalkonsum. Nachteile treten zutage, wenn Kunden damit konfrontiert werden, dass kein dauerhafter Zugriffsanspruch besteht.

\section{Vergleich: Öffentliche Bibliothek (32 Personen)}

Hier wird überwiegend positiv angemerkt, dass es keine Einschränkungen mehr beim Ausüben der Lesetätigkeit gibt, weil man warten muss, bis man ein bestimmtes Buch lesen kann oder weil die Zeit, die man zum Lesen des Buches hat, vorgegeben ist. Zudem empfinden Kunden es als kognitive Entlastung, nicht mehr an Abgabetermine denken zu müssen. Einige Kunden sehen die Größe des Bestands als vergleichbar mit der von Bibliotheken an oder als größer, wenn die Bibliothek/Bücherei in der Gemeinde eher klein ist. Einige Kunden bewerten den Bestand des Angebots als aktueller als in ihrer örtlichen Bibliothek.

7 Personen stellen fest, dass sie mit dem Angebot wieder (regelmäßiger) Bücher lesen.

„Das erste Mal seit hundert Jahren, dass ich endlich mal wieder lese. Mit dem „Buch“ in der Hosentasche kann ich jede kleine Wartezeit nutzen. Ich habe in den letzten Wochen soviel gelesen wie die ganzen letzten Jahre nicht." (571)

„Hab mein Hobby wieder gefunden! Zugunsten meiner Familie fielen zuletzt Bücher oft weg. Dank readfy kann ich jetzt endlich wieder lesen (...).Hab die APP seit 5 Wochen und schon mein 4. Buch angefangen. Für die Kinder waren Bücher dabei, und Kochbücher für mich. Meine Listen sind so voll, dass ich mich ein Jahr lang beschäftigen könnte" (128)

Der zweite Kommentar deutet an, dass nicht allein raumzeitliche Faktoren der Bereitstellung (Ubiquität) die Rückkehr zur Lesetätigkeit befördern, sondern auch andere buchbezogene Aktivitäten. Insbesondere das Stöbern in Büchern und das Entdecken interessanter Titel scheint als eine mit dem Bücherlesen funktional verbundene Tätigkeit eine wichtige Rolle zu spielen und den Nährboden für die Umsetzung der Verhaltensabsicht zu bilden. Auch Köcher zeigt in ihrer Untersuchung von Leserückkehrern, dass das Entdecken eines interessanten Buches der wichtigste Grund für die Wiederaufnahme der Lesetätigkeit ist (vgl. Kap. 3.3).

Kosten-Buchlesemengen-Verhältnis: Ja, 52 Personen (nur Skoobe-Kunden) denken darüber nach, ob sich die Angebotsnutzung finanziell lohnt gemessen am eigenen Leseverhalten. 45 davon bezeichnen sich selbst als Vielleser und betrachten alle eine hohe Leseintensität als Voraussetzung, um keine finanziellen Nachteile durch die Angebotsnutzung zu erleiden. 4 Personen beziehen sich konkret auf die 
Anzahl an gekauften Büchern pro Monat und ihren Kaufpreis als Bewertungs- bzw. Vergleichsmaßstab. 3 Kunden stufen ihre Leseintensität als zu gering ein.

Sparpotenzial: Ja, in 81 Aussagen wägen Kunden ab, ob das Angebot ihnen hilft, weniger Geld für Bücherlesen auszugeben. Bei der Angebotsform Kauf bewerten 66 von 67 Kunden das Angebot als hilfreich beim Sparen (57 Skoobe-Kunden). Bei der Angebotsform Leihe kehrt sich das Verhältnis um: 5 von 6 Personen sehen kein Sparpotenzial gegenüber öffentlichen Bibliotheken.

\subsection{Methodenkritik}

Die Definition von Kategorien und die Entwicklung eines Kategoriensystems, das sich als Erhebungsinstrument eignet, gestaltete sich schwierig und war sehr zeitaufwändig. Zunächst blieb offen, ob mit einer Definition und den Ankerbeispielen auch alle Aussagen gefunden werden können. Hier lieferte der Reliabilitätstest mit zwei anderen Codierern zusätzliche Sicherheit und wichtige Anregungen. Die Arbeit mit Kundenkommentaren und der Versuch, mit den daraus gewonnen Daten Annahmen zu bestätigen, birgt ein Risiko, da die Bearbeiterin keinen eigenen Beitrag zur Datengenerierung leistet und im Vorfeld offen ist, ob das Material geeignet ist, die Untersuchungsfragen zu beantworten: „Finde ich, was ich suche?“ Daher erfolgten für die deduktive Kategorienbildung eine intensive theoretische Auseinandersetzung mit dem Untersuchungsgegenstand und eine Integration der Ergebnisse von zahlreichen qualitativen und quantitativen Studien, die wiederum qualitativ im Sinne der Forschungsfragen analysiert wurden. ${ }^{522}$ Da es sich bei der Stichprobe um keine Zufallsstichprobe handelt, kann kein statistischer Inferenzschluss auf die Grundgesamtheit (alle Kunden von Vermietungsangeboten für E-Books) gezogen werden. ${ }^{523}$ Somit ist das Vorgehen explorativ und die Generalisierbarkeit der Ergebnisse limitiert. ${ }^{524}$ Trotzdem erlauben die qualitativen Daten Rückschlüsse auf Kundenerwartungen. ${ }^{525}$ Der Vorteil der gewählten Methode ist, dass nicht - wie z. B. in Befragungen - Merkmale vorgegeben, sondern dass alle Aspekte vom Kunden selbst zur Sprache gebracht werden und sie somit einen Ausschnitt aus der Kundenwirklichkeit darstellen. Heinonen et al. weisen darauf hin, dass neue Methoden nötig sind, um die Kundenlogik sowie Situationen, in denen Value-in-Use entsteht, erfassen zu können. ${ }^{526}$ Das Kundenurteil bezieht sich - anders als bei klassischen UsabilityStudien - auf die Angebotsnutzung unter natürlichen Bedingungen. ${ }^{527}$ Die Gewinnung dieser Art von Daten ist für die Beantwortung der Forschungsfragen nötig und auch Jacob et al. ziehen in dieser Hinsicht ein positives Fazit ihrer qua-

\footnotetext{
${ }^{522}$ Vgl. Kuckartz (2014), S. 15.

${ }^{523}$ Schnell/ Hill/ Esser (2011), S. 259 f.

${ }^{524}$ Vgl. Jacob/Bruns/Sievert (2013), S. 45.

525 Vgl. auch Wolf (2014), S. 153.

${ }^{526}$ Vgl. Heinonen/ Strandvik (2015), S. 480; vgl. Heinonen/ Strandvik/ Voima (2013), S. 116.

527 Vgl. auch Wolf (2014), S. 152.
} 
litativen Studie: „Die Inhaltsanalyse scheint also in besonderem Maße für die zukünftige Annäherung an die Value in Context Thematik geeignet. “528

${ }^{528} \mathrm{Jacob} /$ Bruns/Sievert (2013), S. 45. 



\section{Zusammenfassung der Ergebnisse und Schluss}

Ziel dieser Arbeit war es, die drei forschungsleitenden Fragen zu beantworten, um Anbietern konkrete Empfehlungen im Hinblick auf eine kundenorientierte Angebotsgestaltung geben zu können und Leistungen aufzudecken, für die potenziell eine Zahlungsbereitschaft besteht.

1. Forschungsleitende Frage: Gibt es Qualitätsmerkmale bei der Bereitstellung von Büchern, die grundsätzlich wichtig für Buchleser sind?

Durch die theoretisch erarbeiteten Qualitätsmerkmale der Bereitstellung, bei der neben qualitativen Studien auch repräsentative Untersuchungen einbezogen wurden, und ihrer empirischen Bestätigung bei einer Stichprobe von Kunden zweier Vermietungsangebote für E-Books, liefert diese Arbeit einen ersten Anhaltspunkt dafür, dass Qualitätsmerkmale von allgemeiner Natur existieren, die für Kunden wichtig sind, wenn sie Bücher erwerben.

Im Allgemeinen schätzen Kunden es, wenn sie selbstbestimmt lesen können, d. h. wenn das Angebot ihnen die Wahl lässt wie viel, wie lange und wann sie lesen. Der Anbieter kann die Freiheit aber bewusst einschränken und die Folgen als Steuerungsinstrument in Bezug auf Kosten und Umsatz je nach Erlös- und Tarifmodell einsetzen, da nicht alle Beschränkungen als stark nachteilig erlebt werden bzw. Anbieter ggf. höhere Zahlungsbereitschaften abschöpfen können. Beim Buchbestand eines Angebots ist offenbar weniger die absolute Anzahl an Titeln wichtig für das Qualitätsurteil, sondern eher das Merkmal der Übereinstimmung mit persönlichen Präferenzen. Je nach Leseverhalten können unterschiedliche Merkmale des Bestands relevant sein, z. B. die Vielfalt der Bucharten oder das Vorhandensein fremdsprachiger Bücher. Konkrete Suchvorgaben (Buchtitel, Autor, Neuerscheinung) können die persönliche Passung bzw. Eignung des Bestands verhindern ebenso wie ein hohes Anspruchsniveau. Eine hohe Reversibilität fördert wiederum die Wahrscheinlichkeit (überraschender) positiver Leseerfahrungen, da Kunden z. B. Bucharten und Autoren evaluieren und lesen, die für sie neuartig sind. Weitere Qualitätsmerkmale können zudem eine regelmäßige Bestandserweiterung und das Gefühl sein, sich darauf verlassen zu können, dass Titel nicht wieder aus dem Bestand entfernt werden. Ferner kann sich eine kundenorientierte Strukturierung des Bestands positiv auf das Qualitätsurteil auswirken, da diese die wahrgenommene Komplexität reduziert und so den Such- und Auswahlprozess erleichtert. Hier spielt sowohl die Tiefe der Strukturierung eine Rolle (Anzahl an Kategorien) als auch die Art der Strukturierung. Bei den Kategorien, die der Anbieter zur Unterteilung nutzt, bewerten Kunden vermutlich, ob es sich um solche handelt, die sie selbst zur Eingrenzung nutzen z. B. Sprache (deutsch oder fremdsprachig), Gefühle beim Lesen 
und Frauenfiguren/ Männerfiguren/ Minderheiten. Interessante Beispiele in diesem Zusammenhang stellen die Kategorien in der Amazon Buchhandlung in Seattle dar: „Comics mit starken Frauen als Hauptfigur oder Bücher für Menschen, die bereit sind, mal anständig zu heulen, Bücher für Eltern, denen Unordnung egal ist“ 529 Darüber hinaus kann in das Qualitätsurteil von Kunden einfließen, wie leicht sie sich in physischen Räumen bzw. in einem softwarebasierten Angebot orientieren können und wie schnell sie Bücher und Funktionen finden und erreichen. Ferner teilt sich ein Teil der Kunden in der Stichprobe ihren kostenpflichtigen Zugang mit Familienmitgliedern. Allerdings ist für jede Person ein eigenes Konto nötig, damit der Vorteil der persönlichen Anpassung (Merklisten, Anzeige der Ergebnisse eigener Aktivitäten etc.) erhalten bleibt. Damit kann eine spezielle Tarifoption für Familien ein Qualitätsmerkmal sein, für das ggf. sogar eine Zahlungsbereitschaft besteht. Als Beispiel lässt sich die Tarifoption „Duo-Ausweis für zwei Erwachsene“ der Stadtbibliothek Leipzig anführen ${ }^{530}$ oder auch die Familien- bzw. Haushaltsbibliothek für Amazon-Kunden. ${ }^{531}$ Bei den finanziellen Kosten, die für den Zugriff auf die Bücher anfallen, bewerten Kunden in der Stichprobe die absolute Preishöhe nicht allein für sich, sondern vor dem Hintergrund ihrer Kostenerfahrungen mit anderen Angebotsformen (Kauf im Handel, Leihe in Bibliothek) und sie prüfen, ob ihnen ein Angebot hilft, im Vergleich zu diesen weniger Geld für das Bücherlesen auszugeben. Bietet ein Angebot Sparpotenzial, so lässt sich dies als kundenspezifischer Vorteil auffassen. Der finanzielle Belastungsgrad eines Tarifs unterscheidet sich je nach Leseintensität, d.h. für Vielleser lohnt sich ein fixer Pauschalbetrag und für Durchschnittsleser eher nicht. Sofern sich die Angebotsnutzung finanziell lohnt, ist dieser Umstand ebenfalls als kundenspezifischer Vorteil zu werten. Die empirische Untersuchung zeigt, dass Kunden in der Stichprobe ihr Leseverhalten realistisch einschätzen und eher nicht zur Überschätzung der Buchlesemenge neigen. Um den Bedürfnissen beider Lesegruppen gerecht zu werden, können Anbieter sowohl einen Tarif anbieten, bei dem mit jeder Transaktion Kosten anfallen, und zum anderen einen Flatrate-Tarif, sodass Kunden sich flexibel entsprechend ihrer situativen Lebensumstände wie Urlaub oder stressigen Phasen im Beruf für den gerade passenden Tarif entscheiden können. Allerdings wurde die Wahrnehmung von transaktionsabhängigen und -unabhängigen Tarifen auf dem Buchmarkt noch nicht erforscht. Ein sogenannter Selbstdisziplinierungseffekt beim Flatrate-Tarif in Verbindung mit einem Abonnement konnte in der Stichprobe nicht nachgewiesen werden. Als mögliche Erklärung wird vorgeschlagen, dass die Kunden des kostenpflichtigen und abonnementbasierten Vermietungsangebotes bereits Vielleser sind und über eine hohe Lesemotivation verfügen. Ein Qualitätsmerkmal, das Kunden in der Stichprobe erwähnen, ist die Entkopplung des Lesens von finanziellen Kosten.

\footnotetext{
${ }^{529}$ Schmieder (2016).

${ }^{530}$ Vgl. Leipziger Städtische Bibliotheken (2015b).

531 Vgl. Amazon (o. J.): Familienbibliothek.
} 
Vermutlich hat dies einen positiven Effekt auf das erlebte Lesevergnügen, jedoch besteht hier ebenfalls noch Forschungsbedarf.

In Bezug auf die finanziellen Kosten positionieren Kunden in der Stichprobe das Flatrate-Mietmodell zwischen Kauf (Miete günstiger) und Leihe (Miete teurer). Hieraus lässt sich ableiten, dass bei Kunden, die bereits Erfahrungen mit kostenpflichtigen Mietangeboten gesammelt haben, drei verschiedene Angebotsformen mental repräsentiert sind. Diese haben, wenn ihr Profil klar umrissen bleibt, aus Kundensicht vermutlich alle ihre Daseinsberechtigung. Bei werbefinanzierten (kostenlosen) Angeboten setzen Kunden sich verstärkt mit ihrem eigenen Beitrag zur Finanzierung des Angebots auseinander. Für Anbieter, die ein solches Erlösmodell verwenden, ist die Frage entscheidend, wie Werbung im Buch von Kunden erlebt wird. In der Stichprobe ist das Verhältnis zwischen Kunden, die Werbung als kognitiv belastend und solchen, die Werbung nicht als störend empfinden, relativ ausgeglichen. Allerdings gilt dies nur für reizarme Werbeformen wie statische Bannerwerbung. Animierte Werbung und Pop-Up-Fenster lösen überwiegend negative Emotionen aus, die letztlich zum Leseabbruch führen. Der Grad der wahrgenommenen kognitiven Belastung hängt vermutlich neben der Werbeform auch von der Buchart, von Lesezielen sowie vom Leseerleben ab. Um hierzu belastbare Aussagen treffen zu können, sind weitergehende Untersuchungen nötig. Es lässt sich jedoch festhalten, dass es Lesern bei hohem Involvement leichter fällt, die Transparenz der Benutzungsschnittstelle aufrechtzuerhalten.

Für Kunden kann ferner eine hohe raumzeitliche Flexibilität, die ein Angebot gewährt, ein Qualitätsmerkmal sein. Der Abruf über das Internet ist der schnellste Weg, ein Buch zu erhalten, aber Kunden müssen hierfür das E-Book als neue Technologie akzeptieren. Bei den Bereitstellungsverfahren Web-Browser und WebCloud ist eine ergänzende Offline-Lese-Option (Download) sowie ein unbeschränkter Offline-Lesezeitraum ein Qualitätsmerkmal und ggf. eine Leistung, für die eine Zahlungsbereitschaft besteht. Wenn Anbieter den Zugriff der Bücher über einen Web-Browser anbieten, umgehen sie Kompatibilitätsprobleme, sodass sie den technisch erreichbaren Kundenkreis erhöhen können. Weiterhin kann es ein Qualitätsmerkmal sein, wenn sich die Ausführung des Programms nicht nachteilig auf Rechenleistung, Speicherplatz und Akkubetrieb eines Geräts auswirkt. Wenn Kunden mehrere Geräte zum Lesen verwenden möchten, kann es ferner ein Qualitätsmerkmal sein, wenn der Anbieter dies technisch ermöglicht. Durch die Zuordnung der Merkmale zu Dimensionen wurde in Anlehnung an Saxer eine Definition der Bereitstellungsqualität entwickelt, die für Anbieter bei konzeptionellen Arbeitsschritten nützlich sein kann:

Die Bereitstellungsqualität ist das Ergebnis eines Kundenurteils, in das einfließt, unter welchen technologischen Bedingungen, in welchem zeitlichen und räumlichen Umfang, in welcher inhaltlichen Vielfalt und Ordnung und zu welchen Kosten Bücher zugänglich sind und wie leicht das Angebot zu benutzen ist.

Als vorteilhaft stellte sich das Vorgehen heraus, etablierte Angebotsformen für gedruckte Bücher (v. a. Sortimentsbuchhandel und öffentliche Bibliotheken) mit den 
Erlebnissen von Kunden bei Vermietungsangeboten für E-Books zu vergleichen, da zahlreiche Parallelen zutage traten, aber auch Punkte, bei denen sich die Angebotsformen voneinander unterscheiden und die helfen können, das Profil von Angebotsformen aus Kundensicht zu schärfen. Allerdings limitieren die Stichproben der gewonnenen Daten (sowohl im theoretischen als auch im empirischen Teil der Arbeit) die Gültigkeit der Qualitätsmerkmale. Zum Beispiel bleibt offen, ob sie auch bei Angeboten im B2B-Kontext sowie bei wissenschaftlichen und ausbildungs-/ berufsbezogenen Büchern ihre Relevanz behalten. Auch bleibt offen wie vollständig der Merkmalskatalog ist.

2. Forschungsleitende Frage: Welche Aktivitäten haben sich im Alltag von Buchlesern etabliert und welche buchbezogenen Aktivitäten können speziell in Online-Angeboten ausgeübt werden?

Die Aktivitäten Lesen, Suchen und Bücher organisieren sind die am häufigsten thematisierten Aktivitäten in der Stichprobe. Aus diesem Befund lässt sich die Annahme ableiten, dass diese auch die größte Bedeutung im Alltag besitzen (Häufigkeit der Ausübung). Eine Empfehlung für Anbieter könnte daher sein, sich zunächst auf die Unterstützung dieser Aktivitäten zu fokussieren. Da für einen Teil der Kunden die Ergebnisse der Aktivitäten anderer Kunden (z. B. Rezensionen, Bewertungen, Schlagworte/ Tags) bei der Suche und der Auswahl von Büchern hilfreich sind, bietet es sich für eine kundenorientierte Gestaltung an, solche Funktionen in ein OnlineAngebot zu integrieren und Maßnahmen zu entwickeln, wie Kunden zur Ausübung der Aktivitäten animiert werden können. Insgesamt verläuft die Aktivitätsentwicklung von Kunden stufenweise. Erst durch die Aneignung von Werkzeugen für die Auswahl von Büchern und zum Navigieren sind Kunden auch innerlich (affektiv) betroffen, was der erste Schritt ist auf dem Weg, solche Inhalte selbst zu erstellen. Alternativ könnten Anbieter über Schnittstellen die von Kunden erzeugten Daten anderer Angebote, z. B. von Buchcommunitys, importieren. Allerdings steigt der Wert eines Angebots für Kunden und damit auch seine Zahlungsbereitschaft, umso aktiver Kunden sind, sodass es aus Anbietersicht sinnvoll sein kann, Kunden zur Nutzung von Funktionen und Werkzeugen aufzufordern. Die Veröffentlichung von eigenen Aktivitäten in einem Online-Angebot erfordert zusätzlich die Bereitschaft zur Selbstoffenbarung. Da diese nicht bei allen Kunden (sofort) gegeben ist, sind Privatsphäre-Einstellungen nötig. Zudem ist es sinnvoll, den Schwierigkeitsgrad einer Aktivität nach dem kreativen Input zu unterscheiden, den Kunden leisten müssen. Die Vorgabe von Buttons und Skalen seitens des Anbieters sind stark anleitend und Kunden müssen nicht kreativ sein, um ihre Meinung auszudrücken. Dies erhöht die Wahrscheinlichkeit der Funktionsnutzung. Für Kunden stellt es hingegen eine Herausforderung dar, ihre Gedanken selbst $\mathrm{zu}$ formulieren. Daher ist es empfehlenswert, Funktionen mit unterschiedlichem Schwierigkeitsgrad technisch unabhängig voneinander zu implementieren, sodass die Aktivitäten separat ausgeführt werden können. 
3. Forschungsleitende Frage: Mit welchen Funktionen können Anbieter von Büchern ihre Kunden bei buchbezogenen Aktivitäten unterstützen?

In der Arbeit zeigt sich, dass ein Anbieter seine Kunden auf vielfältige Weise durch Einstellungsmöglichkeiten (Customization) und automatische Anpassung an ihr Verhalten (Personalisierung v. a. mittels Algorithmen) unterstützen kann. $90 \%$ Kundenkommentare in der Stichprobe, die Optimierungspotenziale thematisieren, beziehen sich auf Customization und der Rest auf Personalisierung. Die Kunden in der Stichprobe sind sich über potenzielle Anwendungsfelder von Personalisierung offenbar wenig im Klaren. Sie äußern stattdessen eher Aspekte, bei denen sie selbst etwas anpassen können, um Aktivitäten angenehmer zu gestalten. Hieraus lässt sich für Anbieter die Empfehlung ableiten, (neue) kundenorientierte Leistungen nicht nur auf Basis von Kundenaussagen zu entwickeln. Optimierungspotenziale beim Lesen bzw. beim Umgang mit dem Buchinhalt betreffen typografische Einstellungen sowie die Unterstützung von Lese- und Suchstrategien. Es lässt sich zudem eine instrumentelle von einer kosmetischen Customization abgrenzen, die v. a. Ausdruck persönlicher Vorlieben ist. Die Unterscheidung kann für Anbiete hilfreich sein, um die (Weiter-)Entwicklung von (neuen) Funktionen sinnvoll priorisieren zu können. Beispielsweise betrachten einige Kunden in der Stichprobe eine Textsuchmaschine und Zoomen als grundlegende Funktionen einer Lesesoftware. Eine beliebte Personalisierungsfunktion ist der automatische Aufruf der letzten Lesestelle. Optimierungspotenzial bei der Suche bieten explorative Navigationswerkzeuge wie Tagclouds, Merklisten von anderen Kunden, Themenlisten und Listen, die algorithmisch erzeugt werden wie „am häufigsten geöffnete Bücher“ oder „am besten bewertete Bücher“. Sie lassen sich auch im Laden zur Sortimentsbildung einsetzen. Zum Beispiel greift die Amazon-Buchhandlung in Seattle auf die Ergebnisse des gemeinsames Online-Filterprozesses von Kunden zurück: „In diesem Laden ist kein Buch zu finden, das weniger als 100 Bewertungen hat und mit weniger als 4,5 Sternen ausgezeichnet ist. “532

Was der Kunde im Suchprozess benötigt, ist abhängig von seiner Suchaufgabe. Beispielsweise wird bei konkreten Suchvorgaben in virtuellen Angeboten eine Suchmaschine benötigt. Explorative Navigationswerkzeuge werden hingegen gebraucht, wenn Kunden z. B. Bücher zu einem bestimmten Thema finden möchten sowie im Falle einer Analogie-Suche oder zum Stöbern. In physischen Räumen machen die natürlichen Verbindungen zwischen Büchern das Stöbern bequem möglich, während diese in virtuellen Angeboten erst angelegt werden müssen. Anbieter können Kunden die Suchstrategie Scanning durch Customization erleichtern z. B. indem unerwünschte Themen/ Bucharten dauerhaft ausgeblendet oder Präferenzen ausgedrückt werden können. Beim Explorieren von Trefferlisten einer Suchmaschine sowie beim Scannen von Kategorien kann es aus Kundensicht nützlich sein, über reichhaltige Informationen zu Büchern zu verfügen ohne separate

\footnotetext{
${ }^{532}$ Schmieder (2016).
} 
Buchdetailseiten aufzurufen. Zudem wünschen sich einige Kunden in der Stichprobe Filter- und Sortiermöglichkeiten. Um bei Kunden die Wahrscheinlichkeit von Sucherfolgen zu steigern (Bücher finden, die mit eigenen Präferenzen übereinstimmen), können Anbieter versuchen, die Nutzung explorativer Navigationswerkzeuge zu fördern.

Explorative Navigationswerkzeuge, die sich leicht benutzen lassen, sind auch ein Mittel, um Leseabbrecher wieder in Kontakt mit Büchern zu bringen. Da eine funktionale Verbindung zwischen den Aktivitäten Auswahl und Lesen besteht, ist eine erfolgreiche Buchauswahl der erste Schritt auf dem Weg zum Lesen. Kunden müssen in der Lage sein, selbst Bücher zu finden, die sie lesen möchten, damit sie die Lesetätigkeit eigenständig aufrechterhalten können.

Um ihre Leseaktivitäten organisieren zu können, ist es hilfreich für Kunden Merk- und Leselisten anlegen zu können. Sie dienen als Erinnerungshilfe oder dazu, um in Lesesituationen z. B. unterwegs schnell Bücher zu finden, die sie selbst lesen oder Kindern vorlesen möchten. Da Kunden aktiv mit ihren Listen umgehen, entwickeln sie klare Vorstellungen davon, wie sich diese leichter verwalten und durchsuchen lassen, was Anbieter für die Entwicklung von Funktionen aufgreifen können. Darüber hinaus schätzen Kunden es vermutlich, eine Übersicht über eigene Aktivitäten zu erhalten (systemgenerierte Interaktionshistorie). Kunden erhalten so die Möglichkeit, die Ergebnisse ihres Tuns als Input in anderen Aktivitäten zu verwenden z. B. ihre Notizen und Lesezeichen oder das Wissen, welche Bücher sie bereits gelesen oder evaluiert haben. Die Visualisierung spiegelt zudem die investierte Arbeit wider und ermöglicht Wirksamkeitserleben, das ein menschliches Grundbedürfnis befriedigt. Wenn Kunden sich über Aktivitäten ausgewählter Kunden oder über Empfehlungen/Neuigkeiten des Anbieters und anderer Akteure informieren lassen können (Abo-Funktion), werden sie bequem auf interessante Bücher aufmerksam.

Insgesamt legen Anbieter durch ihre Gestaltungsentscheidungen (und operativen Marketingmaßnahmen) fest, auf welches Leseverhalten ein Angebot ausgerichtet wird und somit auch, welche Lesetypen sich vom Angebot angesprochen fühlen. Somit kann eine Marktsegmentierung nach dem Leseverhalten der Kunden sinnvoll sein.

In der Arbeit wurden Verlage ausgeblendet, obwohl ihre Kooperationsbereitschaft insbesondere für Anbieter von Miet- und Leih-Modellen u. a. aufgrund lizenzrechtlicher Regelungen eine wichtige Rolle spielt. Aus Anbietersicht ist es nötig, Verlage und Leser für das unterschiedliche Leseverhalten zu sensibilisieren. Bisher wird das Kaufmodell stark fokussiert, da hier das Umsatzpotenzial pro Buch am höchsten ist, allerdings verzichtet ein wachsender Anteil der Bevölkerung und speziell Buchleser darauf, Bücher für sich selbst zu kaufen. Beispielsweise ist für Leser der temporäre Erwerb attraktiv, wenn sie erwarten, ein Buch nur einmal zu lesen oder wiederkehrend, aber in einem begrenzten Zeitraum, sodass Verlage Gefahr laufen, statt geringerer nutzungsabhängiger Erlöse gar keine Erlöse zu erzielen, wenn Leser deshalb auf den Kauf verzichten. Bisher sind kommerzielle, temporäre Angebotsformen auf dem Buchmarkt kaum etabliert, jedoch deutet auch 
der gesellschaftliche Trend „Nutzung statt dauerhafter Besitz“ an, dass das Kundeninteresse an solchen Modellen wachsen wird. 



\section{Literaturverzeichnis}

\section{Bibliographie Forschungsliteratur}

Aellig, Steff (2004): Über den Sinn des Unsinns: Flow-Erleben und Wohlbefinden als Anreize für autotelische Tätigkeiten. Eine Untersuchung mit der Experience Sampling Method (ESM) am Beispiel des Felskletterns, Münster: Waxmann Verlag GmbH

Ahlert, Dieter (1996): Distributionspolitik: das Management des Absatzkanals, 3. Aufl., Stuttgart u. a.: G. Fischer

Ahlert, Dieter / Olbrich, Rainer (1999): Institutionelle Handelsbetriebslehre, 2. Aufl., Münster: MDHD

Albers, Sönke/ Herrmann, Andreas (Hrsg.) (2007): Handbuch Produktmanagement, 3. Aufl., Wiesbaden: Betriebswirtschaftlicher Verlag Dr. Th. Gabler

Arnim, Bernd von/ Knilli, Friedrich (1966): Gewerbliche Leihbüchereien. Berichte, Analysen und Interviews, Gütersloh: C. Bertelsmann Verlag

Bächle, Thomas Christian/ Thimm, Caja (Hrsg.) (2014): Mobile Medien - mobiles Leben. Neue Technologien, Mobilität und die mediatisierte Gesellschaft, Münster: Lit

Bandura, Albert (1977): Self-efficacy: Toward a Unifying Theory of Behavioral Change, in: Psychological Review, Jg. 84, Nr. 2, S. $191-215$

Barth, Klaus/ Hartmann, Michaela/ Schröder, Hendrik (2015): Betriebswirtschaftslehre des Handels, 7. Aufl., Wiesbaden: Springer Gabler

Beck, Klaus (2013): Kommunikationswissenschaft, 3. Aufl., Konstanz: UVK Verlagsgesellschaft

Bentele, Günter/ Brosius, Hans-Bernd/ Jarren, Otfried (Hrsg.) (2013): Lexikon Kommunikations- und Medienwissenschaft, 2. Aufl., Wiesbaden: Springer VS

Blanck, Sandra (2006): Wert- und Wirkungsmessung in Bibliotheken, in: Fulrott/ Krauß-Leichert/ Schütte (Hrsg.): Neues für Bibliotheken - Neues in Bibliotheken. Wiesbaden: Dinges \& Frick, S. $9-105$

Blumenthal, Brad/ Gornostaev, Juri/ Unger, Claus (Hrsg.) (1995): Human-Computer Interaction, Lecture Notes in Computer Science, Heidelberg: Springer

Boll-Westermann, Susanne/ Maaß, Susanne/ Malaka, Rainer (Hrsg.) (2013): Mensch \& Computer 2013, München: Oldenbourg Verlag

Bonfadelli, Heinz/ Fritz, Angela/ Köcher, Renate (Hrsg.): Leseerfahrungen und Lesekarrieren (Lesesozialisation Band 2), Gütersloh: Bertelsmann Stiftung

Bonfadelli, Heinz/ Jarren, Otfried/ Siegert, Gabriele (2010): Einführung in die Publizistikwissenschaft, 3. Aufl., Bern u. a.: Haupt

Bonfadelli, Heinz/Fritz, Angela (1993): Lesen im Alltag von Jugendlichen, in: Bonfadelli/ Fritz/ Köcher (Hrsg.): Leseerfahrungen und Lesekarrieren, Gütersloh: Bertelsmann Stiftung, S. 10 - 213 
Börsenverein (Hrsg.) (2005): Buchkäufer und Leser, Frankfurt a.M.: Börsenverein des Deutschen Buchhandels

Börsenverein (Hrsg.) (2008): Buchkäufer und Leser II. Profile, Motive, Wünsche, Frankfurt a.M.: Börsenverein des Deutschen Buchhandels

Börsenverein (Hrsg.) (2015): Buch und Buchhandel in Zahlen, Frankfurt a.M.: Börsenverein des Deutschen Buchhandels

Börsenverein (Hrsg.) (2015): Buchkäufer, Buchleser 2015. Profile, Motive, Einstellungen, Frankfurt a.M.: Börsenverein des Deutschen Buchhandels

Braun, Ilja (2014): Grundeinkommen statt Urheberrecht? Zum kreativen Schaffen in der digitalen Welt, Bielefeld: transcript

Brosius, Hans-Bernd (2013): Stichwort Leserschaftsforschung, in: Bentele/ Brosius/ Jarren (Hrsg.): Lexikon Kommunikations- und Medienwissenschaft, Wiesbaden: Springer VS

Brosius, Hans-Bernd/ Haas, Alexander/ Koschel, Friederike (2012): Methoden der empirischen Kommunikationsforschung. Eine Einführung, 6. Aufl., Wiesbaden: VS Verlag für Sozialwissenschaft

Bruhn, Manfred/ Hadwich, Karsten (Hrsg.) (2012): Customer Experience, Wiesbaden: Springer Gabler

Bruhn, Manfred/ Hadwich, Karsten (Hrsg.) (2015): Interaktive Wertschöpfung durch Dienstleistungen. Strategische Ausrichtung von Kundeninteraktionen, Geschäftsmodellen und sozialen Netzwerken, Wiesbaden: Springer Fachmedien

Casimir, Torsten (2009): Das Buch stirbt schon wieder nicht! Lesen und Buchkauf in Deutschland, Mainz: Stiftung Lesen, S. 86-92

Charlton, Michael/ Pette, Corinna (1999): Lesesozialisation im Erwachsenenalter. Strategien literarischen Lesens in ihrer Bedeutung für Alltagsbewältigung und Biographie, in: Groeben (Hrsg.): Lesesozialisation in der Mediengesellschaft, Tübingen: Max Niemeyer Verlag GmbH, S. 103 - 117

Dahinden, Urs/ Trappel, Josef (2010): Mediengattungen und Medienformate, in: Bonfadelli/ Jarren/ Siegert (2010): Einführung in die Publizistikwissenschaft, Bern u. a.: Haupt, S. $433-475$

Dahl, Darren W./ Moreau, C. Page (2007): Thinking inside the Box: Why Consumers Enjoy

Constrained Creative Experiences, in: Journal of Marketing Research, Jg. 44, Nr. 3, S. 357 - 369

Davis, Fred D. (1989): Perceived Usefulness, Perceived Ease of Use, and User Acceptance of InformationTechnology, in: MIS Quarterly, Jg. 13, Nr. 3, S. $319-340$

Diao, Fangfang/ Sundar, S. Shyam (2004): Orienting Response and Memory for Web Advertisements: Exploring Effects of Pop-Up Window and Animation, in: Communication Research, Jg. 31, Nr. 5, S. $537-567$

Dürscheid, Christa (2016): Einführung in die Schriftlinguistik, Göttingen: Vandenhoeck \& Ruprecht

Eggert, Martin/ Fließ, Sabine (2015): Service Value aus Kundensicht - Kundenaktivitäten als Ausgangspunkt, in: Bruhn/ Hadwich (Hrsg.): Interaktive Wertschöpfung durch Dienstleistungen, Wiesbaden: Springer Fachmedien, S. $113-132$ 
Eichner, Susanne (2007): Die Sims: sozialverträglich und beziehungsorientiert?, in: tv diskurs, Jg. 11, Nr. 4, S. $60-65$

Engesser, Sven (2013): Die Qualität des Partizipativen Journalismus im Web. Bausteine für ein integratives theoretisches Konzept und eine explanative empirische Analyse, Wiesbaden: Springer VS

Engeström, Yrjö (2008): Entwickelnde Arbeitsforschung. Die Tätigkeitstheorie in der Praxis, Berlin: Lehmanns Media

Fandel, Günter/ Fließ, Sabine/ Jacob, Frank (Hrsg.) (2011): Kundenintegration 2.0., Zeitschrift für Betriebswirtschaft, Special Issue, 5, Wiesbaden: Gabler Verlag

Fließ (Hrsg.): Beiträge zur Dienstleistungsmarketing-Forschung. Aktuelle Forschungsfragen und Forschungsergebnisse, Wiesbaden: Gabler Verlag

Fließ, Sabine / Haase, Michaela / Jacob, Frank / Ehret, Michael (Hrsg.): Kundenintegration und Leistungslehre. Integrative Wertschöpfung in Dienstleistungen, Solutions und Entrepreneurship, Wiesbaden: Springer Fachmedien

Fließ, Sabine/ Dyck, Stefan/ Schmelter, Mailin (2014): Mirror, mirror on the wall - how customers perceive their contribution to service provision, in: Journal of Service Management, Jg. 25, Nr. 4, S. $433-469$

Fließ, Sabine/ Dyck, Stefan/ Schmelter, Mailin/ Volkers, Maarten J. D. (2015): Kundenaktivitäten in Dienstleistungsprozessen - die Sicht der Konsumenten, in: Fließ et al. (Hrsg.): Kundenintegration und Leistungslehre. Integrative Wertschöpfung in Dienstleistungen, Solutions und Entrepreneurship, Wiesbaden: Springer Fachmedien, S. 181- 204.

Fließ, Sabine/ Wittko, Ole/ Schmelter, Mailin (2012): Der Service Experience Value - Stand der Forschung, Konzeptualisierung und empirische Messung, in: Bruhn/ Hadwich (Hrsg.): Customer Experience, Wiesbaden: Springer Gabler, S. 161-184

Foscht, Thomas/ Swoboda, Bernhard/ Schramm-Klein, Hanna: Käuferverhalten. Grundlagen Perspektiven - Anwendungen, 5. Aufl., Wiesbaden: Springer Gabler

Frankenberger, Rudolf/ Haller, Klaus (Hrsg.) (2004): Die moderne Bibliothek. Ein Kompendium der Bibliotheksverwaltung, München: Saur

Frauendorf, Janine (2006): Customer Processes in Business-to-Business Service Transactions, Wiesbaden: Deutscher Universitäts-Verlag

Freyer, Ulrich (2013): Medientechnik. Basiswissen Nachrichtentechnik, Begriffe, Funktionen, Anwendungen, München: Carl Hanser Verlag

Friedrichsen, Mike/ Mühl-Benninghaus, Wolfgang/ Schweiger, Wolfgang (Hrsg.) (2006): Neue Technik, neue Medien, neue Gesellschaft? Ökonomische Herausforderungen der Onlinekommunikation, München: Reinhard Fischer

Früh, Werner (2015): Inhaltsanalyse, 8. Aufl., Konstanz u. a.: UVK Verlagsgesellschaft mbH

Fuchs, Reinhard (2007): Körperliche Aktivität und die Macht der Gewohnheit, in: Fuchs/ Göhner/ Seelig (Hrsg.): Aufbau eines körperlich-aktiven Lebensstils, S. 3 - 22

Fuchs, Reinhard/ Göhner, Wiebke/ Seelig, Harald (Hrsg.) (2007): Aufbau eines körperlichaktiven Lebensstils: Theorie, Empirie und Praxis, Göttingen u. a.: Hogrefe Verlag GmbH \& Co. KG 
Fulrott, Rolf/ Krauß-Leichert, Ute/ Schütte, Christoph-Hubert (Hrsg.) (2006): Neues für Bibliotheken - Neues in Bibliotheken. Wiesbaden: Dinges \& Frick GmbH

Garbe, Christine/ Holle, Karl/ Jesch, Tatjana (2009): Texte lesen. Textverstehen - Lesedidaktik Lesesozialisation, 2. Aufl., Paderborn: Verlag Ferdinand Schöningh GmbH \& Co. KG

Gärtner, Karin (2012): Analyse von Recommendersystemen in Deutschland. Literaturstudie, Wiesbaden: Verlag Dinges \& Frick GmbH

Gassmann, Oliver/ Frankenberger, Karolin/ Csik, Michaela (2013): Geschäftsmodelle entwickeln. 55 innovative Konzepte mit dem St. Galler Business Model Navigator, München: Carl Hanser Verlag

Gehrau, Volker (2003): (Film-) Genres und die Reduktion von Unsicherheit, in: Medien \& Kommunikationswissenschaft (M\&K), Jg. 51, Nr. 2, S. $213-231$

Gerlach, Jin (2014): Die Akzeptanz elektronischer Bücher. Eine umfassende Analyse der Einflussfaktoren, Wiesbaden: Springer Gabler

Göpfert, Herbert G. (Hrsg.) (1975): Lesen und Leben, Frankfurt a.M.

Graef, Ralph Oliver (2016): Recht der E-Books und des Electronic Publishing, München: Verlag C.H. Beck oHG

Graf, Werner (2004): Der Sinn des Lesens. Modi der literarischen Rezeptionskompetenz, Münster: Lit

Groeben, Norbert (Hrsg.) (1999): Lesesozialisation in der Mediengesellschaft. Ein Schwerpunktprogramm, Internationales Archiv für Sozialgeschichte der deutschen Literatur, 10. Sonderheft, Tübingen: Max Niemeyer Verlag GmbH

Hagenhoff, Svenja (2015): Verlage und Buchhandel als Organisationen zur Bereitstellung von Lektüre, in: Rautenberg/ Schneider (Hrsg.): Lesen, Berlin u. a.: Walter de Gruyter, S. 525 - 349, Seitenzahlen gemäß PDF (vorher EPUB)

Hagenhoff, Svenja/Kuhn, Axel (2015): Klickst Du noch oder liest Du schon? Softwarebasierte Benutzungsschnittstellen als Chance und Risiko digitaler Buch-, Zeitschriften- und Zeitungsangebote, in: Pagel (Hrsg.): Schnittstellen (in) der Medienökonomie, Baden-Baden: Nomos Verlagsgesellschaft, S. $217-223$

Håkansson, Maria/ Rost, Mattias/ Holmquist, Lars Erik (2007): Gifts from friends and strangers. A study of mobile music sharing, in: Proceedings of the Tenth European Conference on Computer Supported Cooperative Work, S. $311-330$

Haller, Klaus/ Fabian, Claudia (2004): Bestandserschließung, in: Frankenberger/ Haller (Hrsg.): Die moderne Bibliothek, München: Saur, S. $222-261$

Hanbury, Allan/ Kazai, Gabriella/ Rauber, Andreas/ Fuhr, Norbert (Hrsg.) (2015): Advances in Information Retrieval, Springer

Hanekop, Heidemarie/ Wittke, Volker (2006): Die Entwicklung neuer Formen mobiler Kommunikation und Mediennutzung, in: Hagenhoff (Hrsg.): Internetökonomie der Medienbranche, S. $109-138$

Hartmann, Tilo (2006): Die Selektion unterhaltsamer Medienangebote am Beispiel von Computerspielen. Struktur und Ursachen, Köln: von Halem 
Haupt, Joachim/ Grünewald, Lorenz (2014): Vom Produkt zum Produktionsmittel: Was Medienunternehmen von Spotify lernen können, in: Rau (Hrsg.): Digitale Dämmerung. Die Entmaterialisierung der Medienwirtschaft, Baden-Baden: Nomos Verlagsgesellschaft, S. 95 - 114

Haußer, Karl (1995): Identitätspsychologie, Berlin u. a.: Springer-Verlag

Hautzer, Lena/ Lünich, Marco/ Rössler, Patrick (2012): Social Navigation. Neue Orientierungsmuster bei der Mediennutzung im Internet, Baden-Baden: Nomos Verlagsgesellschaft

Heckhausen, Jutta (Hrsg.) (2000): Motivational Psychology of human development. Developing motivation and motivating development, Amsterdam: Elsevier Science b.v.

Heeter, Carrie (1989): Implications of New Interactive Technologies for Conceptualizing Communication, in: Salvaggio/ Bryant (Hrsg.): Media Use in the Information Age, Hillsdale New York: Lawrence Erlbaum Associates, S. 217 - 235

Heinonen, Kristina (2009): The influence of customer activity on e-service value-in-use, in: International Journal of Electronic Business, Jg. 7, Nr. 2, S. $1-24$

Heinonen, Kristina/ Strandvik, Tore (2015): Customer-dominant logic: foundations and implications, in: Journal of Services Marketing, Jg. 29 Nr. 6/7, S. 472 - 484

Heinonen, Kristina/ Strandvik, Tore/ Mickelsson, Karl-Jacob/ Edvardsson, Bo/ Sundström, Erik/ Andersson, Per (2010): A customer-dominant logic of service, in: Journal of Service Management, Jg. 21, Nr. 4, S. $531-548$

Heinonen, Kristina/ Strandvik, Tore/ Voima, Päivi (2013): Customer dominant value formation in service, in: European Business Review, Jg. 25, Nr. 2, S. $104-123$

Hinterhuber, Hans H./ Matzler, Kurt (Hrsg.) (2009): Kundenorientierte Unternehmensführung. Kundenorientierung -Kundenzufriedenheit - Kundenbindung, 6. Aufl., Wiesbaden: Gabler

Holbrook, Morris B. (1999): Introduction to consumer value, in: Holbrook (Hrsg.): Consumer Value, London u. a.: Routledge, S. 1 - 28

Holbrook, Morris B. (Hrsg.) (1999): Consumer Value. A Framework for Analysis and Research, London u. a.: Routledge

Holodynski, Manfred/ Oerter, Rolf (2008): Tätigkeitsregulation und die Entwicklung von Motivation, Emotion, Volition, in: Oerter/ Montada (Hrsg.): Entwicklungspsychologie, Weinheim u. a.: Beltz Verlag, S. $535-571$

Hörstrup, Robert (2012): Anbieterintegration. Ein konzeptioneller Ansatz zur Analyse und Gestaltung kundenseitiger Nutzungsprozesse, Hamburg: Kovač.

Iyer, Rajesh/ Munci, James A. (2009): Purpose and object of anti-consumption, in: Journal of Business Research, Jg.62, S. $160-168$

Jäckel, Michael (2011): Medienwirkungen, 5. Aufl., Wiesbaden: VS Verlag für Sozialwissenschaften

Jacob, Frank/ Bruns, Katherina/ Sievert, Jens (2013): Value in Context - Eine ressourcendynamische Perspektive, in: Schmitz (Hrsg.): Theorie und Praxis des Dienstleistungsmarketing, Wiesbaden: Springer Gabler, S. 27 - 50

Jagow, Ludwig von (2012): Was sind mögliche Hemmnisse für Zeitschriftenabonnements und was macht sie attraktiv?, in: Deutsche Post (Hrsg.): Zukunft des Zeitschriftenabonnements, S. 2433 
Janneck, Monique/ Gussmann, Svenja/ Jandt, Ines/ Teichmann, Franziska (2013): E-Books. Nutzung und Usability, in: Boll-Westermann/ Maßß/ Malaka (Hrsg.): Mensch \& Computer 2013, München: Oldenbourg Verlag, S. $99-108$

Jers, Cornelia (2012): Konsumieren, Partizipieren und Produzieren im Web 2.0: ein sozialkognitives Modell zur Erklärung der Nutzungsaktivität, Köln: von Halem

Jesch, Tatjana (2009): Textverstehen, in: Garbe/ Holle/ Jesch: Texte lesen, Paderborn: Verlag Ferdinand Schöningh, S. $30-102$

Jungnickel, Karin/ Schweiger, Wolfgang (2014): Publikumsaktivität im 21. Jahrhundert - ein theoriegeleitetes Framework, in: Thimm/ Dang-Anh/ Einspänner (Hrsg.): Digitale Gesellschaft Partizipationskulturen im Netz, Münster: Lit, S. 16-40

Juraschko, Bernd (2015): Praxishandbuch Urheberrecht für Bibliotheken und Informationseinrichtungen, Berlin u. a.: Walter de Gruyter $\mathrm{GmbH}$

Kalyanaraman, Sriram „Sri“/ Wojdynski, Bartosz W. (2015): Affording Control How Customization, Interactivity, and Navigability Affect Psychological Responses to Technology, in: Sundar (Hrsg.): The handbook of the psychology of communication technology, New York: John Wiley \& Sons, S. $425-444$

Kalyanaraman, Sriram/ Sundar, S. Shyam (2006): The Psychological Appeal of Personalized Content in Web Portals: Does Customization Affect Attitudes and Behavior?, in: Journal of Communication, Jg. 56, S. $110-132$

Kaptelinin, Victor/ Kuutti, Kari/ Bannon, Liam (1995): Activity theory: basic concepts and applications. A summary of a tutorial given at the east west HCI95 conference, in: Blumenthal et. al. (1995): Human-Computer Interaction, Heidelberg: Springer, S. $189-201$

Kaptelinin, Victor/ Nardi, Bonnie A. (2006): Acting with technology. Activity theory and interaction design, Cambridge u. a.: MIT Press, Seitenzahlen gemäß PDF (vorher EPUB)

Keiderling, Thomas/ Kutsch, Arnulf/ Steinmetz, Rüdiger (Hrsg.) (2007): Buch-Markt-Theorie. Kommunikations- und medienwissenschaftliche Perspektiven. Erlangen: Filos

Kiefer, Marie Luise (2005): Medienökonomik. Einführung in eine ökonomische Theorie der Medien, 2. Aufl., München u.a: Oldenbourg

Klimmt, Christoph/ Blake, Christopher (2012): Selbstwirksamkeitsmaschinen: Motivationsprozesse interaktiver Unterhaltung, in: Reinecke/ Trepte (Hrsg.): Unterhaltung in neuen Medien, Köln: von Halem, S. $65-81$

Klimmt, Christoph/ Hartmann, Tilo (2006): Effectance, Self-Efficacy, and the Motivation to Play Video Games, in: Vorderer/ Bryant (Hrsg.): Playing video games, motives, responses, and consequences, Mahwah u. a.: Lawrence Erlbaum Associates, S. $133-145$

Klimmt, Christoph/ Weinacht, Stefan/ Donner, Bernhard (2009): Netzkompetenz gleich Werberesistenz? Ein Experiment zur Erinnerung von Online-Werbung in Abhängigkeit von der Interneterfahrung, in: MedienWirtschaft, Nr. 1, S. 6-17

Köcher, Renate (1993): Lesekarrieren - Kontinuität und Brüche, in: Bonfadelli/ Fritz/ Köcher (Hrsg.): Leseerfahrungen und Lesekarrieren, Gütersloh: Bertelsmann Stiftung, S. 215 - 310

Kölbl, Carlos (2010): Kulturhistorische Schule, in: Günter/ Mruck (Hrsg.): Handbuch Qualitative Forschung, Wiesbaden: VS Verlag für Sozialwissenschaften, S. 182 - 194 
Konijn, Elly A./ Utz, Sonja/ Tanis, Martin/ Barnes, Susan B. (Hrsg.) (2008): Mediated Interpersonal Communication, New York u. a.: Routledge

Krippendorf, Klaus (2013): Die semantische Wende. Eine neue Grundlage für Design, Basel: Birkhäuser Verlag $\mathrm{GmbH}$

Kroeber-Riel, Werner/ Gröppel-Klein, Andrea: Konsumentenverhalten, 10. Aufl., München: Verlag Franz Vahlen GmbH

Kuckartz, Udo (2014): Qualitative Inhaltsanalyse. Methoden, Praxis, Computerunterstützung, 2. Aufl., Weinheim u. a.: Beltz Juventa

Kuhlen, Rainer/ Semar, Wolfgang/ Strauch, Dietmar (Hrsg.) (2013): Grundlagen der praktischen Information und Dokumentation: Handbuch zur Einführung in die Informationswissenschaft und -praxis, 6. Aufl., Berlin u. a.: de Gruyter Saur

Kuhn, Axel/ Hagenhoff, Svenja (2015): Digitale Lesemedien, in: Rautenberg/ Schneider (Hrsg.): Lesen, Berlin u. a.: Walter de Gruyter, S. 316 - 331, Seitenzahlen gemäß PDF (vorher EPUB)

Lambrecht, Anja/ Skiera, Bernd (2005): Ursachen eines Flatrate-Bias - Systematisierung und Messung der Einflussfaktoren, in: Schmalenbachs Zeitschrift für betriebswirtschaftliche Forschung (ZFBF), Jg. 58, S. $588-617$

Leemans, H./ Stokmans, M. (1991): Attributes used in choosing books, in: Poetics, Jg. 20, S. 487505

Leimeister, Jan Marco (2015): Einführung in die Wirtschaftsinformatik, 12. Aufl., Berlin u. a.: Springer Gabler

Locher, Lieselotte (2003): Die Bibliothekare bitten zur Kasse. Ein Überblick über die Gebühren in Öffentlichen Bibliotheken, in: Buch und Bibliothek (BuB), Jg. 55, Nr. 10/11, S. 637 - 642

Loest, Klaus-G. (1984): Die Videokassette - ein neues Medium etabliert sich. Videotheken aus bibliothekarischer Perspektive, Wiesbaden: Otto Harrassowitz

Lünenborg, Margreth (2013), Stichwort Genre, in: Bentele/ Brosius/ Jarren (Hrsg.): Lexikon Kommunikations- und Medienwissenschaft, Wiesbaden: Springer VS

Mahrt, Merja (2014): Einstellung, in: Wünsch et al.: Handbuch Medienrezeption, Baden-Baden: Nomos Verlagsgesellschaft, S. $113-127$

Mann, Florian (2010): Filmdistribution über internetbasierte Abrufdienste. Gestaltungsoptionen und intermediale Wechselwirkungen, Berlin: epubli

Matzler, Kurt/ Stahl, Heinz K./ Hinterhuber, Hans H. (2009): Die Customer-based View der Unternehmung, in: Hinterhuber/ Matzler (Hrsg.): Kundenorientierte Unternehmensführung, Wiesbaden: Gabler, S. $4-31$

Mayring, Philipp (2015): Qualitative Inhaltsanalyse, Weinheim u. a.: Beltz Verlag

Mey, Günter/ Mruck, Katja (Hrsg.) (2010): Handbuch Qualitative Forschung, Wiesbaden: VS Verlag für Sozialwissenschaften

Mikkonen, Anna/ Vakkari, Pertti (2016): Finding fiction: Search moves and success in two online catalogs, in: Library \& Information Science Research, Jg. 38, S. $60-68$ 
Möller, Jens/ Schiefele, Ulrich (2004): Motivationale Grundlagen der Lesekompetenz, in: Schiefele et al. (Hrsg.): Struktur, Entwicklung und Förderung von Lesekompetenz, Wiesbaden: Verlag für Sozialwissenschaften, S. $101-123$

Mowshowitz, Abbe (1992): On the Market Value of Information Commodities. II. Supply Price, in: Journal of the American Society for Information Science, Jg. 43, Nr. 3, S. $233-241$

Müller, Kerstin Eva/ Schrepp, Martin (2013): Visuelle Komplexität, Ästhetik und Usability von Benutzerschnittstellen, in: Boll-Westermann/ Maßß/ Malaka (Hrsg.): Mensch \& Computer 2013, München: Oldenbourg Verlag, S. $211-220$

Müller-Hagedorn, Lothar (1995): Stichwort Betriebstypen im Einzelhandel, in: Tietz/ Köhler/ Zentes (Hrsg.): Handwörterbuch des Marketing, Stuttgart: Schäffer-Poeschel, S. 238 - 254

Müller-Hagedorn, Lothar/ Toporowski, Waldemar/ Zielke, Stephan (2012): Der Handel. Grundlagen Management - Strategien, 2. Aufl., Stuttgart: Kohlhammer

Naab, Teresa K./ Schlütz, Daniela (2016): Nutzung von Werbung. Selektion und Vermeidung persuasiver Inhalte, in: Siegert/ Wirth/ Weber/ Lischka (Hrsg.) (2016): Handbuch Werbeforschung, Wiesbaden: Springer VS, S. $223-242$

Neuberger, Christoph (2007): Interaktivität, Interaktion, Internet, in: Publizistik, Jg. 52., Nr. 1, S. $33-50$

Neuberger, Christoph (2009): Internet, Journalismus und Öffentlichkeit. Analyse des Medienumbruchs, in: Neuberger/ Nuernbergk/ Rischke (Hrsg.): Journalismus im Internet, Wiesbaden: VS Verlag für Sozialwissenschaften, S. 19- 105

Neuberger, Christoph/ Nuernbergk, Christian/ Rischke, Melanie (Hrsg.) (2009): Journalismus im Internet. Profession - Partizipation -Technisierung, Wiesbaden: VS Verlag für Sozialwissenschaften

Nielsen, Jacob/ Budiu, Raluca (2013): Mobile Usability. Für iPhone, iPad, Android, Kindle, Heidelberg u. a.: mitp

Norman, Don (2016): The Design of Everyday Things. Psychologie und Design der alltäglichen Dinge, München: Verlag Franz Vahlen

Norton, Michael/ Mochon, Daniel/ Ariely, Dan (2012): The IKEA effect: When labor leads to love, in: Journal of Consumer Psychology, Jg. 22, S. $453-460$

Núñez-Valdéz, Edward Rolando et al. (2012): Implicit feedback techniques on recommender systems applied to electronic books, in: Computers in Human Behavior, Jg. 28, S. 1186 - 1193 Nutz, Walter/ Schlögell, Volker (1991): Die Heftroman-Leserinnen und -Leser in Deutschland.

Beiträge zur Erfassung popularkulturaler Phänomene, in: Communications, Jg. 16, Nr. 2, S. 133235

Oerter, Rolf (1999): Psychologie des Spiels. Ein handlungstheoretischer Ansatz, Weinheim u. a.: Beltz

Oerter, Rolf (2000): Activity and motivation. A plea for human frame motivation, in: Heckhausen

(Hrsg.): Motivational Psychology of human development. Developing motivation and motivating development, Amsterdam: Elsevier Science b.v., S. $57-80$

Oerter, Rolf (2008): Kultur, Ökologie und Entwicklung, in: Oerter/ Montada (Hrsg.): Entwicklungspsychologie, Weinheim u. a.: Beltz Verlag, S. 85-116 
Oerter, Rolf (2014): Der Mensch, das wundersame Wesen. Was Evolution, Kultur und Ontogenese aus uns machen, Wiesbaden: Springer Spektrum

Oerter, Rolf/ Montada, Leo (Hrsg.) (2008): Entwicklungspsychologie, 6. Aufl., Weinheim u. a.: Beltz Verlag

Oliver, Richard L. (2015): Satisfaction. A Behavioral Perspective on the Consumer, 2. Aufl., London u. a.: Routledge

Ooi, Kamy/ Liew, Chern Li (2011): Selecting fiction as part of everyday life information seeking, in: Journal of Documentation, Jg. 67, Nr. 5, S. $748-772$

Ozanne, Lucie K./ Ballantine, Paul W. (2010): Sharing as a form of anti-consumption? An examination of toy library users, in: Journal of Consumer Behaviour, Nr. 9, S. $485-498$

Padrutt, Christian (Hrsg.) (1972): Die Zeitung auf dem Weg ins Jahr 2000, St. Gallen: Zollikofer

Pagel, Sven (Hrsg.) (2015): Schnittstellen (in) der Medienökonomie, Baden-Baden: Nomos Verlagsgesellschaft

Peters, Isabella (2013): Benutzerzentrierte Erschließungsverfahren, in: Kuhlen/ Semar/ Strauch (Hrsg.): Grundlagen der praktischen Information und Dokumentation, Berlin u. a.: de Gruyter Saur, S. $229-237$

Plassmann, Engelbert/ Syré, Ludger (2004): Die Bibliothek und ihre Aufgaben, in: Frankenberger/ Haller (Hrsg.): Die moderne Bibliothek, München: Saur, S. 11 - 41

Platte, Hans K. (1965): Soziologie der Massenkommunikationsmittel. Analyse und Berichte, München, Basel: Ernst Reinhardt Verlag

Pohl, Sigrid/ Umlauf, Konrad (2007): Warenkunde Buch. Strukturen, Inhalte und Tendenzen des deutschsprachigen Buchmarkts der Gegenwart, 2. Aufl., Wiesbaden: Harrassowitz

Purper, Guido (2007): Die Betriebsformen des Einzelhandels aus Konsumentenperspektive, Wiesbaden: Deutscher Universitäts-Verlag

Quiring, Oliver/ Schweiger, Wolfgang (2006): Interaktivität - ten years after. Bestandsaufnahme und Analyserahmen, in: Medien \& Kommunikationswissenschaft, Jg. 54, S. 5 - 24

Rau, Harald (Hrsg.) (2014): Digitale Dämmerung. Die Entmaterialisierung der Medienwirtschaft, Baden-Baden: Nomos Verlagsgesellschaft

Rautenberg, Ursula (2015), Stichwort Buchwissenschaft, in: Rautenberg (Hrsg.): Reclams Sachlexikon des Buches, Ditzingen: Reclam Verlag

Rautenberg, Ursula (2015): Reclams Sachlexikon des Buches: Von der Handschrift zum E-Book, Ditzingen: Reclam Verlag

Rautenberg, Ursula (Hrsg.) (2010): Buchwissenschaft in Deutschland (Theorie und Forschung, Band 1), Berlin u. a.: de Gruyter Saur

Rautenberg, Ursula/ Schneider, Ute (Hrsg.) (2015): Lesen. Ein interdisziplinäres Handbuch, Berlin u. a.: Walter de Gruyter

Rautenberg, Ursula/ Wetzel, Dirk (2001): Buch, Tübingen: Niemeyer

Reimer, Ulrich: Empfehlungssysteme, in: Kuhlen/ Semar/ Strauch (Hrsg.): Grundlagen der praktischen Information und Dokumentation, Berlin u. a.: de Gruyter Saur, S. 238 - 251 
Reinecke, Leonard/ Trepte, Sabine (Hrsg.): Unterhaltung in neuen Medien. Perspektiven zur Rezeption und Wirkung von Online-Medien und interaktiven Unterhaltungsformaten, Köln: von Halem

Reinelt, Julia (2015): Zeitungsleser von heute - App-Nutzer von morgen?, Aachen: Shaker Verlag

Reith, Christina (2007): Convenience im Handel, Frankfurt a.M.: Peter Lang GmbH

Rheinberg, Falko/ Vollmeyer, Regina (2012): Motivation, 8. Aufl., Stuttgart: Kohlhammer GmbH

Robbert, Thomas (2013): Dienstleistungstarife und Nutzungsentscheidungen. Empirische Untersuchung zu Tarifen als Mittel zur Selbstkontrolle, Wiesbaden: Springer Gabler

Robbert, Thomas/ Roth, Stefan (2011): Auswirkungen von Tarifwahlentscheidungen auf die Nutzung von Dienstleistungen, in: Fließ (Hrsg.): Beiträge zur DienstleistungsmarketingForschung, Wiesbaden: Gabler Verlag, S. 149 - 174

Ross, Catherine Sheldrick (1999): Finding without seeking. The information encounter in the context of reading for pleasure, in: Information Processing and Management, Jg. 35, S. 783 - 799

Rossmann, Constanze (2011): Theory of reasoned action - Theory of planned behavior, BadenBaden: Nomos Verlagsgesellschaft

Roth, Wolff-Michael (2009): On the Inclusion of Emotions, Identity, and Ethico-Moral Dimensions of Actions, in: Sannino/ Daniels/ Gutiérrez (Hrsg.) (2009): Learning and Expanding with Activity Theory, New York: Cambridge University Press S. 53 - 71

Rudolph, Dominik (2014): YouTube und Fernsehen: Konkurrenz oder Ergänzung? Eine mehrstufige, vergleichende Analyse aus Nutzersicht unter besonderer Berücksichtigung der Digital Natives, Baden-Baden: Nomos Verlagsgesellschaft

Salvaggio, Jerry Lee/ Bryant, Jennings (Hrsg.) (1989): Media Use in the Information Age. Emerging patterns of adoption and consumer use, Hillsdale New York: Lawrence Erlbaum Associates

Sannino, Annalisa/ Daniels, Harry/ Gutiérrez, Kris D. (Hrsg.) (2009): Learning and Expanding with Activity Theory, New York: Cambridge University Press

Saxer, Ulrich (1972): Zukünftige Funktionskonstellationen der Spezialmedien (Zeitschrift, Buch, Wort- bzw. Tonträger, Film, audio-visuelle Systeme) in: Padrutt (Hrsg.): Die Zeitung auf dem Weg ins Jahr 2000, St. Gallen: Zollikofer, S. $123-145$

Saxer, Ulrich (1975): Das Buch in der Medienkonkurrenz, in: Göpfert (Hrsg.): Lesen und Leben, Frankfurt a.M.: S. $206-243$

Saxer, Ulrich (2010): Buchwissenschaft als Medienwissenschaft, in: Rautenberg (Hrsg.): Buchwissenschaft in Deutschland, Berlin u. a.: de Gruyter Saur, S. 65 - 104

Saxer, Ulrich (2012): Mediengesellschaft. Eine kommunikationssoziologische Perspektive, Wiesbaden: VS Verlag für Sozialwissenschaften

Schedl, Markus/ Flexer, Arthur/ Urbano, Julián (2013): The neglected user in music information retrieval research, in: Journal of Intelligent Information System, Jg. 41, Nr. 3, S. $523-539$

Schey, Sabine/ Rieder, Christian (2014): Wie Smartphones das Leben in einer Gesellschaft verändern - Ergebnisse einer qualitativen Untersuchung der Smartphone-Nutzung in der Schweiz, in: Bächle/ Thimm (Hrsg.): Mobile Medien - Mobiles Leben, Münster: Lit, S. 195 - 217 
Schiefele, Ulrich/ Artelt, Cordula/ Schneider, Wolfgang/ Stanat, Petra (Hrsg.) (2004): Struktur, Entwicklung und Förderung von Lesekompetenz. Vertiefende Analysen im Rahmen von PISA 2000, Wiesbaden: Verlag für Sozialwissenschaften

Schmidt, Jan (2011): Das neue Netz. Merkmale, Praktiken und Folgen des Web 2.0, 2. Aufl., Konstanz: UVK Verlagsgesellschaft $\mathrm{mbH}$

Schmitz, Gertrud (Hrsg.) (2013): Theorie und Praxis des Dienstleistungsmarketing. Aktuelle Konzepte und Entwicklungen, Wiesbaden: Springer Gabler

Schnauber, Anna/ Wolf, Cornelia (2016): Media habits and their impact on media platform selection for information use, in: Studies in Communication/ Media (SCM), Jg. 5, Nr. 1, S. $107-127$

Schnell, Rainer/ Hill, Paul B./ Esser, Elke (2011): Methoden der empirischen Sozialforschung, München: Oldenbourg Wissenschaftsverlag $\mathrm{GmbH}$

Schönbach, Klaus (2005): „Das Eigene im Fremden“ Zuverlässige Überraschung: Eine wesentliche Medienfunktion?, in: Publizistik, Jg. 50, Nr. 3, S. $344-352$

Schönbach, Klaus (2009): Wertvoller Service. Bücherlesen als „zuverlässige Überraschung“, in: Stiftung Lesen (Hrsg.): Lesen in Deutschland 2008, Mainz: Stiftung Lesen, S. 57 - 63

Schumann, Christina (2013): Der Publikumserfolg von Computerspielen. Qualität als Erklärung für Selektion und Spielerleben. Baden-Baden: Nomos Verlagsgesellschaft

Schweiger, Wolfgang (2007): Theorien der Mediennutzung. Eine Einführung. Wiesbaden: VS Verlag für Sozialwissenschaften

Schweiger, Wolfgang (2010): Informationsnutzung online: Informationssuche, Selektion, Rezeption und Usability von Online-Medien, in: Schweiger/ Beck (2010): Handbuch Online-Kommunikation, Wiesbaden: VS Verlag für Sozialwissenschaften, S. $184-210$

Schweiger, Wolfgang/ Beck, Klaus (2010): Handbuch Online-Kommunikation, Wiesbaden: VS Verlag für Sozialwissenschaften

Schweiger, Wolfgang/ Quiring, Oliver (2006): User-Generated Content auf massenmedialen Websites - eine Systematik, in: Friedrichsen/ Mühl-Benninghaus/ Schweiger (Hrsg.), Neue Technik, neue Medien, neue Gesellschaft?, München: Reinhard Fischer, S. 97-120

Siegert, Gabriele/ Wirth, Werner/ Weber, Patrick/ Lischka, Juliane A. (Hrsg.) (2016): Handbuch Werbeforschung, Wiesbaden: Springer VS

Silberer, Günter (2009): Kundenkenntnis im Handel - Operationalisierung, Quellen und Wirkungen im Überblick, in: Silberer (Hrsg.): Kundenkenntnis im Handel. Ausprägungen, Herkunft und Wirkungen, Göttingen: Universitätsverlag Göttingen, S. 15 - 40

Silberer, Günter (Hrsg.) (2009): Kundenkenntnis im Handel. Ausprägungen, Herkunft und Wirkungen, Göttingen: Universitätsverlag Göttingen

Skiera, Bernd/ Lambrecht, Anja (2007): Erlösmodelle im Internet, in: Albers/ Herrmann (Hrsg.): Handbuch Produktmanagement, Wiesbaden: Betriebswirtschaftlicher Verlag Dr. Th. Gabler, S. $869-886$

Sommer, Christoph/ Rimscha, M. Bjørn von (2014): Jenseits von traditionellen Mediengattungen. Die transmediale Angebotsmatrix, in: Rau (Hrsg.): Digitale Dämmerung. Die Entmaterialisierung der Medienwirtschaft, Baden-Baden: Nomos Verlagsgesellschaft, S. 245 - 264 
Spitzmüller, Jürgen (2016): Typoraphie, in: Dürscheid: Einführung in die Schriftlinguistik, S. 209242

Stark, Birgit/ Dörr, Dieter/ Aufenanger, Stefan (Hrsg.) (2014): Die Googleisierung der Informationssuche. Suchmaschinen zwischen Nutzung und Regulierung, Berlin: de Gruyter.

Stark, Birgit/ Magin, Melanie/ Jürgens, Pascal (2014): Navigieren im Netz - Befunde einer qualitativen und quantitativen Nutzerbefragung, in: Stark/ Dörr/ Aufenanger (Hrsg.): Die Googleisierung der Informationssuche, Berlin: de Gruyter, S. $20-74$

Stiftung Lesen (Hrsg.) (2009): Lesen in Deutschland 2008. Eine Studie der Stiftung Lesen, Mainz

Sundar, S. Shyam (2008): Self as source. Agency and customization in interactive media, in:

Konijn/ Utz/ Tanis/ Barnes (Hrsg.): Mediated Interpersonal Communication, New York u. a.: Routledge, S. 58 - 74

Sundar, S. Shyam/ Bellur, Saraswathi/ Oh, Jeeyun/ Jia, Haiyan/ Kim, Hyang-sook (2016): Theoretical Importance of Contingency in Human-Computer Interaction: Effects of Message Interactivity on User Engagement, in: Communication Research, Jg. 43, Nr. 5, S. $595-625$

Sundar, S. Shyam/ Marathe, Sampada S. (2010): Personalization versus Customization: The Importance of Agency, Privacy, and Power Usage, in: Human Communication Research, Jg. 36,

S. $298-322$

Sundar, Shyam S. (Hrsg.) (2015): The handbook of the psychology of communication technology, New York: John Wiley \& Sons

Sundar, Shyam S./ Jia, Haiyan/ Waddell, T. Frankling/ Huang, Yan (2015): Toward theory of interactive media effects (TIME). Four models for explaining how interface features affect user psychology, in: Sundar (Hrsg.): The handbook of the psychology of communication technology, New York: John Wiley \& Sons, S. $47-86$

Taddicken, Monika/Schenk, Michael (2011): Selbstoffenbarung und Privatsphäre im Social Web, in: Wolling/ Will/ Schumann (Hrsg.): Medieninnovationen, in: Konstanz: UVK Verlagsgesellschaft, S. $319-332$

Tesar, Verena (2013): Online-Verleihmodelle: Wie Bibliotheken und andere Anbieter E-Books über das Internet verleihen können, München: GRIN Verlag GmbH

Thimm, Caja/ Dang-Anh, Mark/ Einspänner, Jessica (Hrsg.) (2014): Digitale Gesellschaft - Partizipationskulturen im Netz, Münster: Lit

Tietz, Bruno/ Köhler, Richard Köhler/ Zentes, Joachim (Hrsg.) (1995): Handwörterbuch des Marketing, 2. Aufl., Stuttgart: Schäffer-Poeschel

Tietzel, Manfred (1995): Literaturökonomik, Tübingen: Mohr Trepte, Sabine/ Reinecke, Leonard (2010): Unterhaltung online - Motive, Erleben, Effekte, in: Schweiger/ Beck (2010): Handbuch Online-Kommunikation, Wiesbaden: VS Verlag für Sozialwissenschaften, S. $211-233$

Umlauf, Konrad (2015): Stichwort Leihbibliothek, in: Rautenberg (Hrsg.): Reclams Sachlexikon des Buches, Ditzingen: Reclam Verlag

Vassiliou, Magda/ Rowley, Jennifer (2008): Progressing the definition of „e-book“, in: Library Hi Tech, Jg. 26, Nr. 3, S. $355-368$

Naab, Teresa K. (2013): Gewohnheiten und Rituale der Fernsehnutzung. Theoretische Konzeption und methodische Perspektiven. Baden-Baden: Nomos Verlagsgesellschaft 
Vorderer, Peter/ Bryant, Jennings (Hrsg.) (2006): Playing video games. Motives, responses, and consequences, Mahwah u. a.: Lawrence Erlbaum Associates

Wagner, Hans (2008): Qualitative Methoden in der Kommunikationswissenschaft. Ein Lehr- und Studienbuch, München: Verlag Reinhard Fischer

Walter, Benedikt von (2007): Intermediation und Digitalisierung. Ein ökonomisches Konzept am Beispiel der konvergenten Medienbranche, Wiesbaden: Deutscher Universitäts-Verlag

Wang, Hsiau-Han (2013): Orientierung in bekannten und unbekannten Ladenumwelten. Theorie, Befunde und Implikationen, Wiesbaden: Springer Gabler

Weiber, Rolf/ Hörstrup, Robert/ Mühlhaus, Daniel (2011): Akzeptanz anbieterseitiger Integration in Alltagsprozesse der Konsumenten: Erste empirische Ergebnisse, in: Fandel/ Fließ/ Jacob (Hrsg.): Kundenintegration 2.0., Zeitschrift für Betriebswirtschaft, Special Issue 5, Wiesbaden: Gabler Verlag, S. $111-146$

Werth, Lioba/ Mayer, Jennifer (2008): Sozialpsychologie, Berlin u. a.: Springer-Verlag

Weyers, Stefan/ Sujbert, Monika/ Eckensberger, Lutz H. (2007): Recht und Unrecht aus kindlicher Sicht. Die Entwicklung rechtsanaloger Strukturen im kindlichen Denken und Handeln, Münster u. a.: Waxmann Verlag GmbH

Wirth, Werner \& Schweiger, Wolfgang (1999): Selektion neu betrachtet. Auswahlentscheidungen im Internet, in: Wirth/ Schweiger (Hrsg.): Selektion im Internet, Opladen: Westdeutscher Verlag, S. $43-70$

Wirth, Werner \& Schweiger, Wolfgang (Hrsg.) (1999): Selektion im Internet. Empirische Analysen zu einem Schlüsselkonzept, Opladen: Westdeutscher Verlag.

Wirth, Werner/ Schramm, Holger (2010): Medienrezeptionsforschung, in: Bonfadelli/ Jarren/ Siegert (2010): Einführung in die Publizistikwissenschaft, Bern u. a.: Haupt, S. 575 - 603

Wirtz, Bernd W. (2013): Medien- und Internetmanagement, 8. Aufl., Wiesbaden: Springer Gabler

Wolf, Cornelia (2014): Mobiler Journalismus. Angebote, Produktionsroutinen und redaktionelle

Strategien deutscher Print- und Rundfunkredaktionen, Baden-Baden: Nomos Verlagsgesellschaft

Wolling, Jens/Will, Andreas/Schumann, Christina (Hrsg.) (2011): Medieninnovationen. Wie Medienentwicklungen die Kommunikation in der Gesellschaft verändern, Konstanz: UVK Verlagsgesellschaft

Wünsch, Carsten/ Schramm, Holger/ Gehrau, Volker/ Bilandzic, Helena (2014): Handbuch Medienrezeption, Baden-Baden: Nomos Verlagsgesellschaft

Zech, Herbert (2014): Lizenzen für die Benutzung von Musik, Film und E-Books in der Cloud. Das Verhältnis von Urheber- und Vertragsrecht bei Verträgen über den Werkkonsum per CloudComputing, in: Zeitschrift für Urheber- und Medienrecht, Jg. 58, Nr. 1

Zeckert, Patricia F. (2007): Ein alter Unbekannter. Das Phänomen des ungelesenen Buches, in: Keiderling/ Kutsch/ Steinmetz (Hrsg.): Buch-Markt-Theorie, Erlangen: Filos, S. $153-180$. 


\section{Periodika/ Fachpresse Buchbranche}

\section{Buchreport (URLs)}

Ausgelesen (2016), buchreport.de, 21. 09. 2015, abrufbar unter: https://www.buchreport.de/2015/09/ 21/ausgelesen-3/ (Gesehen am 06.09. 2016)

Bei Tablets gibt es kaum Differenzierungsmöglichkeiten (2014), Interview mit Michael Tamblyn und Taka Aiki, buchreport.de, 21. 10. 2014, abrufbar unter: https://www.buchreport.de/2014/10/21/ bei-tablets-gibt-es-kaumdifferenzierungsmoeglichkeiten/ (Gesehen am 06. 09. 2016)

Bonnier gibt E-Books für Onleihe frei (2016), buchreport.de, 10. 05. 2016, abrufbar unter: https:// www.buchreport.de/2016/05/10/bonnier-gibt-e-books-fuer-onleihe-frei/ (Gesehen am 06. 09. 2016)

Flatrate unter Druck (2016), buchreport.de, 19. 02. 2016, abrufbar unter: https://www.buchreport.de/ 2016/02/19/flatrate-unter-druck/ (Gesehen am 06. 09. 2016)

Kivelip, Lucy (2011): „Das Lesen attraktiver gestalten“, buchreport.de, 29. 09. 2011, abrufbar unter https://www.buchreport.de/2011/09/29/das-lesen-attraktiver-gestalten/ (Gesehen am 06. 09. 2016)

Nutzen oder Schaden? (2016), in: buchreport.de, 25. 01. 2016, abrufbar unter: https://www.buchre port.de/2016/01/25/nutzen-oder-schaden/ (Gesehen am 06.09. 2016)

Tolino zieht am Kindle vorbei (2014), buchreport.de, 14. 11. 2014, abrufbar unter: https://www.buch report.de/2014/11/14/tolino-zieht-am-kindle-vorbei/ (Gesehen am 06. 09. 2016)

\section{Buchreport (gedruckt)}

Lenz, Daniel (2014): Die riskante Wette auf neue Wachstumsimpulse, in: buchreport.magazin, Nr. 7, S. $60-65$

Verkauf vs. Onleihe (2015), in: Buchreport.express, Nr. 27, S. 8

\section{Online-Quellen (URLs):}

Amazon (o. J.): Über die Familienbibliothek, Hilfe und Kundenservice, amazon.de, abrufbar unter: https://www.amazon.de/gp/help/customer/display.html?nodeId=201620400 (Gesehen am 11. 09. 2016)

AWA (2011): Sport, Freizeit, Kultur, Bücher, Sprachen, in: Allensbacher Marktanalyse Werbeträgeranalyse, Auszug Codebuch, S. 59 - 63, abrufbar unter: http://www.ifd-allensbach.de/filead min/AWA/AWA2011/AWA2011_Inhalt_Codebuchausschnitte/AWA_201 1_Codebuch_Kultur_-Buecher__Sprachen.pdf (Gesehen am 06. 09. 2016)

AWA (2013): Sport, Freizeit, Kultur, Bücher, Sprachen, in: Allensbacher Marktanalyse Werbeträgeranalyse, Auszug Codebuch, S. 56 - 60, abrufbar unter: http://www.ifd-allensbach.de/filead min/AWA/AWA2013/Codebuchausschnitte/AWA_2013_Codebuch_Kultu r_Buecher_Spra chen.pdf (Gesehen am 06. 09. 2016)

AWA (2014): Kultur, Bücher, Sprachen, in: Allensbacher Marktanalyse Werbeträgeranalyse, Auszug Codebuch, S. 63 -67, abrufbar unter: http://www.ifd-allensbach.de/fileadmin/AWA/AWA2014/ Codebuchausschnitte/AWA2014_Codebuch_Kultur_Buecher_Sprachen.pdf (Gesehen am 06. 09. 2016) 
AWA (2015): Kultur, Bücher, Sprachen, in: Allensbacher Marktanalyse Werbeträgeranalyse, Auszug Codebuch, S. 63 -67, abrufbar unter: http://www.ifd-allensbach.de/fileadmin/AWA/AWA2015/ Codebuchausschnitte/AWA2015_Codebuch_Kultur_Buecher_Sprachen.pdf (Gesehen am 06. 09. 2016)

AWA (2016): Kultur, Bücher, Sprachen, in: Allensbacher Marktanalyse Werbeträgeranalyse, Auszug Codebuch, S. 62 - 66, abrufbar unter: http://www.ifd-allensbach.de/fileadmin/AWA/AWA2016/ Codebuchausschnitte/AWA2016_Codebuch_Kultur_Buecher_Sprachen.pdf (Gesehen am 06. 09. 2016)

Bentley, Frank/ Metcalf, Crysta/ Harboe, Gunnar (2006): Personal vs. Commercial Content. The Similarities Between Consumer Use of Photos and Music, in: Proceedings of the SIGCHI Conference on Human Factors in Computing Systems, abrufbar unter: http://web.mit.edu/bentley/ www/papers/chi06.pdf (Gesehen am 06. 09. 2016)

Bitkom (2013): Nutzung von E-Books in Deutschland, Vortrag von Achim Berg, 01. 10. 2013, abrufbar unter: http://www.bitkom-re-search.de/WebRoot/Store19/Shops/63742557/5249/8360/ACCD/ FE2D/6 A2 A/C0 A8/28B9 /4F2 A/Leseprobe_E_Books_2013.pdf (Gesehen am 06. 09. 2016)

Bitkom (2014): Nutzung von E-Books in Deutschland, Vortrag von Christian Illek, 30. 09. 2014, abrufbar unter: http://www.bitkomre-search.de/WebRoot/Store19/Shops/63742557/544 A/04AA/ C6E6/DF2F/6697/C0 A8/2AB8/ D124/Leseprobe_E_Books_2014.pdf (Gesehen am 06. 09. 2016)

Bitkom (2015): Studie zur Nutzung von E-Books, Vortrag von Achim Berg, 06. 10. 2015, abrufbar unter: https://www.bitkom.org/Presse/Anhaenge-an-PIs/2015/10-Oktober/BITKOM-PKCharts-EBooks-Studie-06-10-2015-final2.pdf (Gesehen am 06. 09. 2016)

Böhringer, Martin/ Gerlach, Lutz (2010): Das Phänomen des Status-Sharings aus tätigkeitstheoretischer Perspektive, in: Proceedings of the 3rd Workshop on 'Digitale Soziale Netze', INFORMATIK 2010, 27.09.-02. 10. 2010, Leipzig, Germany, 2010, abrufbar unter https://tuchemnitz.de/wirtschaft/wi2/publications/boehringer_gerlach_informatik_2010.pdf (Gesehen am 06. 09. 2016)

Brown, Barry A. T./ Geelhoed, Erik/ Sellen, Abigail J. (2001): The Use of Conventional and New Music Media: Implications for Future Technologies, in: Proceedings of Interact, abrufbar unter: http:// research.microsoft.com/en-us/um/people/asellen/publications/conventional\%20music\%2001. pdf (Gesehen am 06.09. 2016)

Buchanan, George/ McKay, Dana (2011): In the Bookshop: Examining Popular Search Strategies, in: Proceedings of the 11th annual international ACM/IEEE joint conference on Digital libraries, abrufbar unter: http://hdl.handle.net/1959.3/195936 (Gesehen am 06. 09. 2016)

BVMI (2016): Musikindustrie in Zahlen 2015, Bundesverband Musikindustrie e. V. (Hrsg.), Berlin, abrufbar unter http://www.musikindustrie.de/fileadmin/piclib/publikationen/BVMI-2015Jahr buch-ePaper.pdf (Gesehen am 08. 04. 2016)

Cunningham, Sally Jo/ Jones, Matt/ Jones, Jones (2004): Organizing digital music for use. An examination of personal music collections, in: Proceedings: Fifth International Conference on Music Information Retrieval. ISMIR 2004 abrufbar unter: http://ismir2004.ismir.net/procee dings/p082-page-447-paper221.pdf (Gesehen am 06. 09. 2016)

Deutsche Post AG (Hrsg.) (2012): Zukunft des Zeitschriftenabonnements. Ergebnisse einer repräsentativen Befragung von Bevölkerung und Führungskräften in deutschen Publikumszeitschriftenverlagen, Bonn, abrufbar unter: https://www.deutschepost.de/content/dam/dpag/ima ges/Z_z/Zukunft-desZeitschriftenabos/Abostudie__.pdf (Gesehen am 06. 09. 2016) 
Diehl, Kristin/ Poynor, Cait (2010): Great Expectations?! Assortment Size, Expectations and Satisfaction, abrufbar unter: https://marketing.wharton.upenn.edu/mktg/assets/file/diehl_poynor_ great_expectations_final. pdf (Gesehen am 06.09. 2016)

Dörr, Jonathan/ Wagner, Thomas/ Benlian, Alexander/ Hess, Thomas (2013): Music as a Service: Eine Alternative für Musikpiraten? Eine empirische Untersuchung zur Nutzungsintention von Streaming-Services für Musik, erschienen in: Wirtschaftsinformatik, Nr. 6, abrufbar unter: http://www.ise.tu-darmstadt.de/media/ise/publikationen_3/MaaS_Artikel_Wirtschaftsinforma tik_2013.pdf (Gesehen am 09.09. 2016)

Drenner, Sara/ Sen, Shilad/ Terveen, Loren (2008): Crafting the initial User Experience to achieve community goals, in: Proceedings of the 2008 ACM conference on Recommender systems, abrufbar unter: http://files.grouplens.org/papers/p187-drenner.pdf (Gesehen am 06. 09. 2016)

El-Heliebi, Chalid (2015): Welcher eReader funktioniert mit der Onleihe? Worauf man achten muss, allesebook.de, 29. 09. 2015, abrufbar unter: http://allesebook.de/anleitung/welcherereader-funk tioniert-mit-der-onleihe-worauf-man-achten-muss-66178/ (Gesehen am 06. 09. 2016)

Engel, Bernhard/ Breunig, Christian (2015): Massenkommunikation 2015: Mediennutzung im Intermediavergleich, in: Media Perspektiven, Nr. 7 - 8, S. 310 -322, abrufbar unter: http://www. ard-werbung.de/fileadmin/user_upload/media-perspektiven/pdf/2015/07082015_Engel_Breu nig.pdf (Gesehen am 06. 09. 2016)

Fühles-Ubach, Simone/Brenner, Simon/Lorenz, Miriam (2014): Den Benutzer aktivieren... Datenauswertung und Analyse der Nutzungsdaten und Online-Befragungen zum Einsatz von „LibraryThing for Libraries“, Bericht zum Kooperationsprojekt der FH Köln mit dem Dezernat 48.08 der Bezirksregierung Düsseldorf, abrufbar unter: http://www.brd.nrw.de/schule/privatschulen_ sonstiges/oeffentl__Biblio__Container/pdf_neu/ 7/LibraryThing-for-Libraries-in-NRW-Projekt bericht-2012-2013_14_05_30.pdf (Gesehen am 06.09.2016)

gfu/ BVT/ GfK (2010): Consumer Electronics Marktindex Deutschland (CEMIX), abrufbar unter http:// www.bvt-ev.de/Downloads/CEMIX/CEMIX_Q1-Q4_2010.pdf?m=1463044879 (Gesehen am 06. 09. 2016)

gfu/ BVT/ GfK (2012): Consumer Electronics Marktindex Deutschland (CEMIX), abrufbar unter http:// www.bvt-ev.de/Downloads/CEMIX/CEMIX_Q1-Q4_2012.pdf?m=1463044878 (Gesehen am 06. 09. 2016)

gfu/ BVT/ GfK (2014): Consumer Electronics Marktindex Deutschland (CEMIX), abrufbar unter http:// www.bvt-ev.de/Downloads/CEMIX/CEMIX_Q1-Q4_2014.pdf?m=1463044877 (Gesehen am 06. 09. 2016)

gfu/ BVT/ GfK (2015): Consumer Electronics Marktindex Deutschland (CEMIX), abrufbar unter http:// www.bvt-ev.de/Downloads/CEMIX/CEMIX_Q1-Q4_2015.pdf?m=1463044876 (Gesehen am 06. 09. 2016)

Graf, Alexander (2014): Zur Zukunft des stationären Buchhandels \# Interview mit Christian Riethmüller, 13. 10. 2014, abrufbar unter https://www.youtube.com/watch?v=j9u7FD1elAA (Gesehen am 06. 09. 2016)

Gummerus, Johanna/ Koskull, Catharina von (Hrsg.) (2015): The Nordic School - service marketing and management for the future, Helsinki: Hanken School of Economics, abrufbar unter: http:// liu.diva-portal.org/smash/get/diva2:822245/FULLTEXT01.pdf (Gesehen am 06. 09. 2016) 
Hagenhoff, Svenja (2014): Digitale Schriftmedien - Eine Melange aus Informationsgut und Software, Erlanger Beiträge zur Medienwirtschaft, Nr. 4, abrufbar unter: https://opus4.kobv.de/opus4fau/ files/5569/EBM+2014_04+Digitale+Lesemedien+Melange.pdf (Gesehen am 06. 09. 2016)

Hagenhoff, Svenja (Hrsg.) (2006): Internetökonomie der Medienbranche, Göttingen: Universitätsverlag Göttingen, abrufbar unter: http://www.univerlag.uni-goettingen.de/bitstream/handle/3/ isbn-3-938616-29-6/mediaconomy_book.pdf?sequence $=1$ (Gesehen am 06. 09. 2016)

Henke, Harold (2002): Survey on electronic book features, The open ebook forum, 05. 04. 2005, abrufbar unter: http://www.cs.unibo.it/ cianca/wwwpages/dd/survey.pdf (Gesehen am 06. 09. 2016)

Hermann, Anastasia/ Lulei, Kristin (2013): Media Trend Outlook. E-Books im Aufwind, Whitepaper, PricewaterhouseCoopers AG Wirtschaftsprüfungsgesellschaft (Hrsg.), abrufbar unter: https:// www.pwc.de/de/technologie-medien-und-telekommunikation/assets/whitepaperebooks.pdf (Gesehen am 06.09. 2016)

Hinze, A./ McKay, D./ Vanderschantz, N./ Timpany, C./ Cunningham, S. J (2012): Book selection behavior in the physical library: implications for ebook collections, in: Proceedings of the 11th annual international ACM/IEEE joint conference on Digital libraries, abrufbar unter: http://rese archbank.swinburne.edu.au/vital/access/services/Download/swin:28487/SOURCE1 (Gesehen am 06.09. 2016)

Hofacker, Charles F./ Goldsmith, Ronald E./ Bridges, Eileen/ Swilley, Esther (2007): EServices: A Synthesis and Research Agenda, abrufbar unter: http://myweb.fsu.edu/chofacker/pubs/Hof acker_Goldsmith_Bridges_Swilley_2007.pdf (Gesehen am 06.09.2016)

Horch, Wolfgang (2014): Hamburger Firma Campusritter vermietet Fachbücher, abendblatt.de, 02. 09. 2014, abrufbar unter: http://www.abendblatt.de/hamburg/article131823501/Hambur gerFirma-Campusritter-vermietet-Fachbuecher.html (Gesehen am 09.09. 2016)

Hüttemann, Christian (2010): Bibliotheksgebühren: Gebührenmodelle, Rahmenbedingungen der Erhebung und Auswirkungen auf die Nutzung. Ein Vergleich verschiedener Öffentlicher Bibliotheken in Nordrhein-Westfalen, unveröffentlichte Bachelorarbeit, Fachhochschule Köln, abrufbar unter: https://epb.bibl.th-koeln.de/files/33/Huettemann_Bachelorarbeit.pdf (Gesehen am 06. 09. 2016)

IfH (2010): Die Zukunft des Deutschen Sortimentsbuchhandels. Gesamtbericht, Studie im Auftrag des Börsenvereins des Deutschen Buchhandels e.V., Köln: Institut für Handelsforschung GmbH (nicht mehr online/ Kopie bei Bearbeiterin)

Institut für Demoskopie Allensbach (Hrsg.) (2016): Die Zukunft der Bibliotheken in Deutschland, Studie im Auftrag der ekz.bibliotheksservice GmbH, abrufbar unter: https://www.ekz.de/filead min/ekz-media/unternehmen/Zukunftsstudie/2016_Studie_Zukunft_Bibliotheken_in_Deutsch land.pdf (Gesehen am 06. 09. 2016)

Jürgens, Pascal/ Stark, Birgit/ Magin, Melanie (2015): Messung von Personalisierung in computervermittelter Kommunikation, in: Maireder / Ausserhofer/ Schumann/ Taddicken (Hrsg.): Digitale Methoden in der Kommunikationswissenschaft, Berlin, abrufbar unter: http://digitalcommu nicationresearch.de/v2/juergens-et-al/ (Gesehen am 07. 09. 2016)

Kaptelinin, Victor (2014): Stichwort Affordances, in: Lowgren et al.: The Encyclopedia of HumanComputer Interaction, abrufbar unter: https://www.interactiondesign.org/literature/book/theencyclopedia-of-human-computer-interaction-2nded/affordances (Gesehen am 06. 09. 2016) 
Kaptelinin, Victor/ Nardi, Bonnie (2012): Affordances in HCI: Toward a Mediated Action Perspective, in: Proceedings of the SIGCHI Conference on Human Factors in Computing Systems, abrufbar unter: http://www.mn.uio.no/ifi/forskning/grupper/design/arrangementer/affordances-oslonov-22a.pdf (Gesehen am 06. 09. 2016)

Kohler, Michael (2007): Gebrauchstauglichkeit und Nutzererwartungen im Online-Shopping. Psychologische Untersuchungen zur Skriptnormierung und Erwartungskonformität beim Einkaufsverhalten im Internet, unveröffentlichte Dissertation, Universität Trier, abrufbar unter: http://ubt.opus.hbz-nrw.de/volltexte/2008/457/pdf/kohler_diss.pdf (Gesehen am 06. 09. 2016)

Koolen, Marijn/ Bogers, Toine/ van den Bosch, Antal/ Kamps, Jaap (2015): Looking for Books in Social Media: An Analysis of Complex Search Requests, in: Hanbury et al. (Hrsg.) (2015): Advances in Information Retrieval, Springer, abrufbar unter: http://humanities.uva.nl/ kamps/publications/ 2015/kool:look15.pdf (Gesehen am 06. 09. 2016)

Kornmeier, Claudia (2016): Ein E-Book streamen? - Bezahl-Abos kommen nicht recht in Gang, heise.de, 14. 03. 2016, abrufbar unter: http://www.heise.de/newsticker/meldung/Ein-E-Bookstre amen-Bezahl-Abos-kommen-nicht-recht-in-Gang-3134806.html (Gesehen am: 08. 04. 2016)

Laplante, Audrey (2010): User's relevance criteria in music retrieval in everyday life. An exploratory study, in: International Society for Music Information Retrieval Conference, abrufbar unter: http://www.mirlab.org/conference_papers/International_Conference/ISMIR\%202010/ISMIR_ 2010_papers/ismir2010-103.pdf (Gesehen am 06. 09. 2016)

Laplante, Audrey/ Downie, J. Stephen (2006): Everyday Life Music Information-Seeking Behaviour of Young Adults, in: Proceedings of the 7th International Conference on Music Information Retrieval, abrufbar unter http://ismir2006.ismir.net/PAPERS/ISMIR06132_Paper.pdf (Gesehen am 07. 09. 2016)

Leipziger Städtische Bibliotheken (2015a): Neu: vorzeitige Rückgabe von E-Books in der Onleihe möglich, stadtbibliothek.leipzig.de, 05. 08. 2015, abrufbar unter: http://stadtbibliothek.leipzig. de/detailansicht-news/news/neu-vorzeitige-rueckgabe-von-ebooks-in-der-onleihe-moeglich/ (Gesehen am 07.08. 2016)

Leipziger Städtische Bibliotheken (2015b): Gebührensatzung der Leipziger Städtischen Bibliotheken, stadtbibliothek.leipzig.de, 01.11. 2015, abrufbar unter: http://stadtbibliothek.leipzig.de/anmel dung-und-benutzung/gebuehren/ (Gesehen am 11. 09. 2016)

Leontjew, Alexei Nikolajewitsch (1977/ 1982): Tätigkeit, Bewußtsein, Persönlichkeit, Köln: Campus, abrufbar unter: http://www.kritische-psychologie.de/texte/al1982.html (Gesehen am 06. 09. 2016)

Lowgren, Jonas et al. (2014): The Encyclopedia of Human-Computer Interaction, 2. Aufl., https:// www.interaction-design.org/literature/book/the-encyclopedia-of-human-computerinteraction2nd-ed (Gesehen am 06. 09. 2016)

Maireder, Axel/ Ausserhofer, Julian/ Schumann, Christina / Taddicken, Monika (Hrsg.) (2015): Digitale Methoden in der Kommunikationswissenschaft, Berlin, abrufbar unter: http://digital communicationresearch.de/v2/ (Gesehen am 07. 09. 2016)

Mander, Jason (2015): Two thirds of Netflixers share their account, globalwebindex.net/blog, 20. 07. 2015, abrufbar unter http://www.globalwebindex.net/blog/two-thirds-of-netflixers-sharetheir-accounts (Gesehen am 20.04. 2015) 
Messerschmidt, Maren/ Naskowski, Maike (2011): Wertvolle Bibliothek!? Wirkungsforschung und Wertmessung in den Stadtbibliotheken Bad Oldesloe und Wedel, unveröffentlichte Masterarbeit, Hochschule für Angewandte Wissenschaften Hamburg, abrufbar unter: http://edoc.sub.unihamburg.de/haw/volltexte/2011/1346/pdf/Messerschmidt_MarenNaskowski_Maike_11_02_25.pdf (Gesehen am 06. 09. 2016)

Mickelsson, Jacob (2014): Customer Activity. A perspective on service use, Helsinki: Springer Fachmedien, abrufbar unter: https://helda.helsinki.fi/bitstream/handle/10138/45016/ 267_978952 - $232-223-4$.pdf?sequence=1 (Gesehen am 04. 09. 2016)

Norman, Donald (1999): Affordance, Conventions and Design, abrufbar unter: http://cseweb.ucsd. edu/ goguen/courses/271sp03/jnd.html (Gesehen am 06. 09. 2016)

Oestreicher-Singer, Gal/ Zalmanson, Lior (2013): Content or Community? A digital business strategy for content providers in the social age, in: MIS Quarterly, Jg. 37, Nr. 2, S. $591-616$, abrufbar unter: http://pages.stern.nyu.edu/ goestrei/MISQ_LastFM_2013.pdf (Gesehen am 06. 09. 2016)

Rauscher, Barbara/ Hess, Thomas (2005): Kontextsensitive Inhaltebereitstellung: Begriffsklärung und Analysegrundlagen, Arbeitspapiere des Instituts für Wirtschaftsinformatik und Neue Medien, Nr. 3, München, abrufbar unter: http://www.wim.bwl.unimuenchen.de/download/epub/ ab_2005_03.pdf (Gesehen am 06. 09.2016)

Rieder, Bernhard (2004): Algorithmische Mediatoren: Software-Agenten als Gegenstand der Medienwissenschaften, in: Medien Journal, Jg. 28, Nr. 1, S. 36 - 46, abrufbar unter: http://rieder.pol sys.net/files/algorithmische_mediatoren_final.pdf (Gesehen am 06. 09. 2016)

Römer, Stefanus/ Freitag, Marcus (2004): Besonderheiten der Anwendungsentwicklung für die mobile Nutzung -ein Leitfaden - Teil 2, in: WissenHeute, Jg. 57, Nr. 12, . 660 - 667, abrufbar unter: http://www.stefanus-roemer.de/media/2004-12.PDF (Gesehen am 06. 09. 2016)

Rückriem, Georg (2014): „Die realisierte Tätigkeit ist reicher, wahrer als das sie vorwegnehmende Bewusstsein.“ Zum Verständnis der Tätigkeit bei Leont'ev., in: Tätigkeitstheorie. Journal für tätigkeitstheoretische Forschung in Deutschland, Heft 10, S. 75 - 108, abrufbar unter http://www. ich-sciences.de/media/journal/Ausgabe_10/Heft_10_2014.pdf (Gesehen am 06. 09. 2016)

Schmoll/ Tiemann/ Welzel (2014): Digitale Mobilität - Dynamik im öffentlichen Raum, Kompetenzzentrum Öffentliche IT Fraunhofer-Institut für Offene Kommunikationssysteme FOKUS (Hrsg.), abrufbar unter: https://www.oeffentliche-it.de/documents/10181/14412/Digitale+Mobilit \%C3 \%A4 t+Dynamik+im+\%C3 \%B6ffentliche n+Raum (Gesehen am 06. 09. 2016)

Schwiering, Bianca (2011): TÜV Saar NetResearch. InSight Buchhandel. Was Kunden wirklich wollen, abrufbar unter: http://www.tuev-netresearch.de/mediapool/105/branchenticker_buchhan del_insight.pdf (Gesehen am 06.09. 2016)

Seefeldt, Jürgen (2011): Öffentliche Bibliotheken, bibliotheksportal.de, Deutscher Bibliotheksverband e.V. (Hrsg.), abrufbar unter: http://www.bibliotheksportal.de/bibliotheken/bibliothekenindeutschland/bibliothekslandschaft/oeffentliche-bibliotheken.html (Gesehen am 06. 09. 2016)

Shatzkin, Mike (2014): Subscription services for ebooks progress to becoming a real experiment, idealog.com, 27. 05. 2014, abrufbar unter: http://www.idealog.com/blog/subscription-servicesebooks-progress-becoming-real-experiment/ (Gesehen am 06. 09. 2016) 
Siegemund, Johanna (2016): Netflixierung des Buches. Lesehäppchen für die Generation Smartphone, Interview mit Svenja Hagenhoff, 11. 02. 2016, abrufbar unter: https://detektor.fm/kultur/ netflixierung-des-buches (Gesehen am 12. 04. 2016)

Strandvik, Tore/ Heinonen, Kristina (2015): Essentials of customer dominant logic, in: Gummerus/ Koskull (Hrsg.): The Nordic School, Helsinki: Hanken School of Economics, S. 111 - 127

Thudt, Alice/ Hinrichs, Uta/ Carpendale, Sheelagh (2015): A Modular Approach to Promote Creativity and Inspiration in Search, in: Proceedings of the ACM Conference on Creativity and Cognition, abrufbar unter: http://www.utahinrichs.de/uta/uploads/Publications/Publications/Thudt2015. pdf (Gesehen am 06. 09. 2016)

Tornack, Christian / Christmann, Stefan / Hagenhoff, Svenja (2011): Tendenzielle Unterschiede zwischen B2B- und B2C-Anwendungen für mobile Endgeräte, Arbeitsbericht Nr. 3, Georg-August-Universität Göttingen, abrufbar unter: http://webdoc.sub.gwdg.de/ebook/serien/lm/arbeits berichte_anwebus/2011_03.pdf (Gesehen am 06.09.2016)

van Eimeren, Birgit/ Ridder, Christa-Maria (2011): Trends in der Nutzung und Bewertung der Medien 1970 bis 2010, in: Media Perspektiven, Nr. 1, S. 2 - 15, abrufbar unter: http://www.ardwerbung.de/fileadmin/user_upload/media-perspektiven/pdf/2011/012011_Eimeren_Ridder.pdf (Gesehen am 06.09. 2016)

Vermeulen, Jo/ Luyten, Kris/ van den Hoven, Elise/ Coninx, Karin (2013): Crossing the Bridge over Norman's Gulf of Execution: Revealing Feedforward's True Identity, in: Proceedings of the SIGCHI Conference on Human Factors in Computing Systems, abrufbar unter: http://jozilla.net/ uploads/Research/VermeulenLuytenVandenHovenConinx_chi2013.pdf (Gesehen am 06. 09. 2016)

Skoobe (2014): eBook-Flatrate-Studie: Skoobe verändert, wie Menschen lesen, Pressemitteilung vom 30. 09. 2014, abrufbar unter: https://www.skoobe.de/press/ebook-flatrate-studie-skoobeveraen dert-wie-menschen-lesen (Gesehen am 06. 09. 2016)

Schmieder, Jürgen (2016): Amazon und die Rückkehr der Bibliothekare, sueddeutsche.de, 07. 08. 2016, abrufbar unter: http://www.sueddeutsche.de/kultur/amazon-buchhandlungen-derduft-der-buecher-1.3111338 (Gesehen am 11. 09. 2016)

Weigl, David M./ Guastavino, Catherine (2011): User studies in the music information retrieval literature, in: 12th International Society for Music Information Retrieval Conference (ISMIR 2011), abrufbar unter: http://ismir2011.ismir.net/papers/OS5-1.pdf (Gesehen am 06. 09. 2016)

Zalmanson, Lior/ Oestreicher-Singer, Gal (2015): „Your Action is Needed“: The Effect of WebsiteInitiated Participation on User Contributions to Content Websites, Working Paper Nr. 2, abrufbar unter: http://aisel.aisnet.org/cgi/viewcontent.cgi?article=1675\&context=icis2015 (Gesehen am 06.09.2016)

Zentes, Joachim/ Freer, Tatjana/ Beham, Frank (2013): Neue Mietkonzepte: Nutzen statt Haben. Potenziale und Herausforderungen für Unternehmen, Institut für Handel \& Internationales Marketing (H.I.MA.) (Hrsg.), Universität des Saarlandes, abrufbar unter: http://www.unisaarland.de/fileadmin/user_upload/Professoren/fr13_ProfZentes/sonstiges/Neue_Mietkonzepte_ Nutzen_statt_Haben.pdf (Gesehen am 06.09. 2016) 
Anhang 



\section{Anhang A Codeplan | „Inhaltsanalyse von Kundenkommentaren zur App von Readfy und Skoobe“}

\section{Einführung}

Ziel der Untersuchung ist es, die Eigenschaften und Einflussfaktoren zu messen, welche die Zufriedenheit der Kunden und das Weiternutzungsverhalten beeinflussen. Der Untersuchung liegt ein Erwartungs-Bestätigungs-Modell zugrunde. Aus den Urteilen der Kunden von Angebotseigenschaften und den Kundenerlebnissen lassen sich Kundenerwartungen ableiten.

\section{Untersuchungsmaterial und Auswahleinheit:}

Ausgewählt wurden die Nutzerkommentare zu den Apps der Anbieter Readfy und Skoobe im Google Play Store (https://play.google.com/store/apps in der Kategorie Bücher \& Nachschlagewerke) und im Amazon App Store (https://www.amazon.de in der Kategorie Spiele und Apps/ Bücher \& Reader). Der Untersuchungsgegenstand befindet sich auf der Produktseite der App im jeweiligen Shop in einem standardisierten Bereich mit der Bezeichnung „Bewertungen“ (Google Play Store) bzw. „Kundenrezensionen“ (Amazon App Store). Die Kommentare beziehen sich auf alle Versionen im Auswertungszeittraum.

Die Kopien der Nutzerkommentare wurden am 01. 03. 2015 (Skoobe-App) und am 04. 10. 2015 (Readfy-App) erstellt und in einer Word-Datei gespeichert. Die App von Readfy wurde inzwischen aus dem Amazon App Store entfernt, sodass die Kommentare nur noch der Bearbeiterin vorliegen. Die Nutzerkommentare werden mit einer fortlaufenden Nummer versehen. Als Auswertungszeitraum wurden 14 Monate bei Skoobe (01.01.2014-28.02.2015) und 16 Monate bei Readfy (01.11. 2014 - 29.02. 2016) gewählt, um ausreichend viel Datenmaterial zu gewinnen. Zu Beginn wurde bei Readfy auch der Monat Oktober im Jahr 2014 aufgenommen. Da dieser zeitlich jedoch mit dem offiziellen Start der App im September 2014 zusammenfällt, beschränken sich die Kommentare im Google Play Store größtenteils auf Support-Anfragen und Hinweise auf Programmierfehler, sodass der Monat wieder verworfen wurde. Nach einem Update im November 2014 lässt sich ein Rückgang der Kommentare, die sich mit technischen Problemen beschäftigen, feststellen. Die Kopie der Nutzerkommentare vom 01.10.2015-29.02.2016 bei Readfy wurde am 11.06. 2016 vorgenommen, um den Verlust des Datenmaterials aus dem Oktober auszugleichen. Insgesamt handelt es sich um eine Auswahl aus einer Grundgesamtheit (alle veröffentlichten Kommentare im Store zum Stichtag). 


\section{Analyseeinheiten}

Die Analyseeinheit in dieser Arbeit bildet ein Kundenkommentar (auch Kundenrezension). Dieser besteht aus

- Name des Verfassers (hier: grauer Balken)

- Benutzerbild des Verfassers (nur Google Play Store)

- Punktbewertung (5-Sterne-Skala)

- Veröffentlichungsdatum im Store/ Datum der letzten Aktualisierung (Tag/Monat/Jahr)

- $\quad$ Text des Verfassers

Überschrift, sofern dieses Feld vom Verfasser ausgefüllt wird. Bei der Browseransicht wird diese fett gesetzt und dem Text vorangestellt, während sie beim Zugang über die Store-App oberhalb des Textes platziert wird (hier: „Fer Umme“). Bei Amazon befindet sich die Überschrift rechts neben der Punkbewertung (hier: „Supergute Sache!")

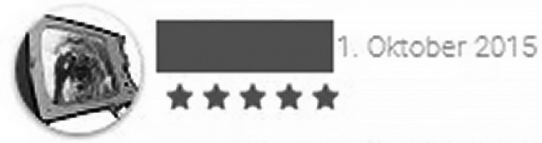

Fer Umme und dafür exellent! Die Werbung holt mich kurz aus der Versenkung, ist aber schnell weckgeklickt.

Abb. A.1 Kundenkommentar als Analyseeinheit im Google Play Store (Browseransicht, 11. 06. 2016)

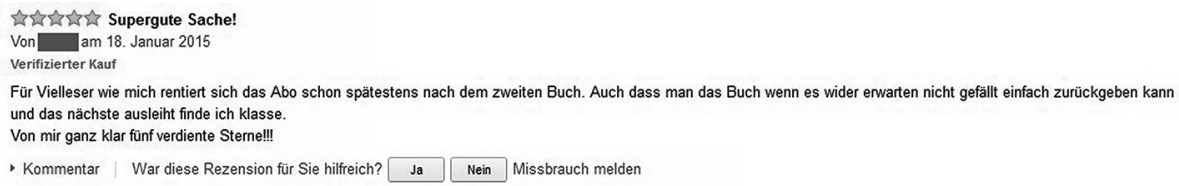

Abb. A.2 Kundenrezension als Analyseeinheit im Amazon App Store (Browseransicht, 11.06. 2016)

Etwaige Reaktionen des Anbieters auf einen Nutzerkommentar gehören hingegen nicht zur Analyseeinheit und wurden nicht gespeichert. Mehr Informationen hierzu unter https://support.google.com/googleplay/android-developer/answer/138230? $\mathrm{hl}=\mathrm{de}$ 
Fer Umme und dafür exellent! Die Werbung

holt mich kurz aus der Versenkung, ist aber

schnell weckgeklickt.

1. Oktober 2015

Vielen Dank für die Bewertung!

Abb. A.3 Beispiel für die Reaktion des Anbieters Readfy („Vielen Dank für die Bewertung!“) auf einen Kundenkommentar im Google Play Store (Browseransicht, 11.06. 2016).

\section{Dimensionen und Kategorien}

Da die Meinung des einzelnen Kunden zu einem Sachverhalt interessiert, stellt der Kundenkommentar die Analyseeinheit dar. Es soll jede Rezension gelesen und jede Kategorie, die inhaltlich oder formal identifiziert werden kann, ein Zahlenwert zugeordnet werden (Codierung). Die Bezugsgröße der Codierung ist formal der abgrenzbare Kundenkommentar. Eine Filtervariable erfasst, ob die formal definierte Einheit der Codierung auch auf semantischer Ebene für die Untersuchung relevant ist (V1 A: Art der Analyseeinheit). Dies ist der Fall, wenn der Bedeutungsgehalt einer Aussage die inhaltliche Definition mindestens einer Kategorie erfüllt und sich somit für eine Messung eignet. Eine Kategorie ist wiederum das Ergebnis einer operationalen Definition, die sicherstellt, dass das Material in Daten überführt werden kann. Die Kategorien bzw. Variablen werden im nachfolgenden Teil ausführlich dargestellt. Der Kunde kann in seinem Kommentar keine Aussage, eine Aussage oder mehrere Aussagen treffen, die inhaltlich für die Untersuchung von Bedeutung sind. Dem Kommentar können demzufolge entweder keine oder unterschiedlich viele Kategorien zugeordnet werden. Es wird davon ausgegangen, dass sich immer dieselbe Person in einem Kommentar äußert, also kein Sprecherwechsel stattfindet. Die so auf formaler und semantischer Ebene definierte Bezugsgröße der Codierung ist mit der Analyseeinheit identisch und das Kategoriensystem soll deshalb pro Kommentar nur 1x angewendet werden. Außerdem ist jeder Kategorie immer nur ein Code zuzuordnen. 


\section{Formale Kategorien/ Variablen}

Formale Bestimmung der Analyseeinheit

$\mathrm{Zu}$ den in dieser Arbeit relevanten Aspekten der Analyseeinheit in dieser Arbeit gehören:

- Kommentartext ohne Berücksichtigung von Emoticons wie :-) oder ;-) und Piktogrammen

- Überschrift

- Datum

- Quelle (Amazon App Store oder Google Play Store).

$\mathrm{Zu}$ codieren sind demnach:

Vo A: App-Anbieter

Skoobe

Readfy

V0B: Shopbetreiber

Google

Amazon

VoC: Monat

metrisch (MM)

VOD: Jahr

metrisch (JJJJ)

VOE: Textumfang

metrisch

Hierzu wird die Funktion „Zählen von Wörtern in einer Auswahl“ in Microsoft Word genutzt.

Siehe https://support.office.com/de-de/article/Z\%C3 \%A4hlen-der-Anzahl-von-W\% C3 \%B6rtern-in-einem-Dokument-3c9e6a11-a04d-43b4-977c-563a0e0d5da3)

Nicht codiert werden Name, Benutzerbild und Punktbewertung. Die Kommentare werden fortlaufend nummeriert, sodass jeder Kommentar eindeutig identifiziert werden kann.

\section{Inhaltliche Kategorien/ Variablen}

Inhaltliche Bestimmung der Analyseeinheit

In der Arbeit soll nicht das gesamte Datenmaterial untersucht werden. Die Analyse eines Kommentars soll nur erfolgen, wenn er sich als gehaltvoll im Hinblick auf die 
Prüfung der Annahmen erweist. Eine Analyse erfolgt somit nicht, wenn nur eine allgemeine Bewertung vorgenommen wird im Sinne von „Super“, „Toll“, „Mist“ ohne weitere Erläuterung. Häufig besteht der Kommentar in solchen Fällen nur aus einem Wort, jedoch kann es sich auch um einen Satz handeln.

Beispiel: Bin sehr mit der APP zufrieden. Daumen hoch

Ebenfalls nicht analysiert werden Kommentare, in welchem die Kunden lediglich dem Anbieter danken oder nur eine Empfehlung aussprechen. Desweiteren soll keine Analyse von Kommentaren erfolgen, die dem Bereich Spam oder Off-Topic zugeordnet werden können (kein Bezug zum Produkt). Bei (indirekten) SupportAnfragen oder Kommentaren, die sich lediglich mit Funktionsproblemen und Programmierfehlern beschäftigen, ist im Einzelfall abzuwägen, ob die Aussage mit einer Kategorie gemessen werden kann oder nicht.

Beispiel für eine Aussage, die keiner Kategorie zugeordnet werden kann und daher nicht relevant ist:

„Man kann sich nicht mal registrieren Scheiße“

\section{V1: Art der Analyseeinheit}

Diese Kategorie ist zu codieren, wenn der Kommentar inhaltlich nicht durch eine Kategorie erfasst werden kann. Diese Variable fungiert somit als Filter. Eine Codierung innerhalb der Dimensionen erfolgt nur wenn gilt: V1 $\neq 0$

0 nicht relevant

1 relevant

\section{Dimension 1: Erreichbarkeit des Angebots}

Unter Erreichbarkeit wird die Möglichkeit verstanden, das Angebot aus technischer Sicht nutzen zu können. Hierunter fallen die Aspekte Netzverbindung und Gerätegebundenheit.

\section{Netzverbindung}

Da es sich um Online-Dienste handelt, ist zunächst einmal eine Netzverbindung nötig, um auf den Dienst und die Funktionen zugreifen zu können. Ubiquität (Überallverfügbarkeit) der Bücher ist gegeben, wenn der Zugriff auf die Daten überall möglich ist. Voraussetzung hierfür ist Gerätemobilität und eine Netzverbindung. Die Netzverbindung wird überflüssig, wenn die Bücher auf das Gerät geladen werden können. Während eine Internetverbindung mehr oder weniger stark mit Wechselhaftigkeit und Unsicherheit verbunden ist, garantiert der Download einen störungsfreien Zugang. Demnach kann es vorteilhaft sein, ein Buch speichern, herunterladen bzw. offline lesen zu können. Dies wird mit Offline-Lesen umschrieben. Von Bedeutung kann in diesem Zusammenhang auch sein, wie lange die Bücher offline gelesen werden können. 
V2 A: Ubiquität und Offline-Lesen

Die Kategorie wird erfasst, wenn Kunden einschätzen, wie wichtig ihnen die Möglichkeit ist, Bücher offline lesen zu können.

Beispiel: „Nur schade dass es lediglich online läuft"

Codiert werden 13 oder 14, wenn Kunden sich mit der Zeitspanne auseinandersetzen, in der sie ohne Netzverbindung lesen können.

Beispiel: „Das einzige, was ich mir wünschen würde wäre, dass die Bücher nach dem Herunterladen länger offline verfügbar sind“

Nicht codiert wird, wenn Kunden lediglich erwähnen, dass eine Netzverbindung nötig ist.

0 nicht erwähnt

1 Offline-Nutzung erwähnt

11 Offline-Nutzung wichtig

12 Offline-Nutzung egal

13 Dauer Offline-Nutzung wichtig

14 Dauer Offline-Nutzung egal

\section{Gerätegebundenheit}

Die Nutzung von Software ist an Geräte gebunden, die für den Betrieb mit Energie versorgt werden müssen. Beim Einsatz von Akkus ist zu berücksichtigen, dass die Betriebszeit des Geräts begrenzt ist. Da die Anbieter keine Browser- oder DesktopVersion für den Dienst bereitstellen, erfolgt die Nutzung der App über mobile Endgeräte. Mobile Endgeräte wie Smartphone, TabletPC und E-Reader sind im Vergleich zu Desktop-PCs und Notebooks kleiner und leichter. Auch weisen die Bildschirme einen geringeren Durchmesser auf. Die Bildschirmtechnologie von EReadern ist im Gegensatz zur LCD-Technik für lange Leseepisoden optimiert und benötigt für die Darstellung keinen Strom. Bei mobilen Endgeräten erfolgen die Eingaben ohne Maus und Tastatur. Stattdessen erlaubt der Touchscreen eine Steuerung mit den Fingern. Desweiteren weisen Smartphone, Tablet-PC und E-Reader geringere Speicherkapazitäten auf sowie niedrigere Rechenleistungen als Desktop-PC und Notebook. Zusammengefasst können Geräte mit folgenden Merkmalen beschrieben werden:

- Größe und Gewicht

- Ausgabe- und Eingabemöglichkeit

- Akkulaufzeit

- Speicherkapazität und Rechenleistung

\section{V3 A: Gerätemerkmale}

Für Kunden spielt es eine Rolle, ob die Entwickler der Software bzw. die Anbieter die grundlegenden Eigenschaften der Endgeräte berücksichtigt haben, auf denen die 
Apps installiert wird. Codiert werden Kommentare, in denen die Kunden die Merkmale Speicherkapazität, Rechenleistung und Akkubetrieb bewerten. Das Urteil kann positiv, negativ oder nicht eindeutig ausfallen.

Beispiel: „Durch die Möglichkeit die Ansicht auf weiße Schrift mit schwarzem Hintergrund einzustellen ist die App sogar sehr verträglich für den Akku“

0 nicht erwähnt

1 Urteil insgesamt positiv

2 Urteil insgesamt negativ

3 Urteil unklar

V3B: Kompatibilitätsprobleme mit Gerätetypen und Betriebssystemen

Entscheidend aus Sicht der Kunden ist, ob sie den Dienst mit den Geräten nutzen können, die sie besitzen (Gerätetypen, Betriebssystem). Häufig ist es eine bewusste Entscheidung der Anbieter, dass der Dienst nur mit bestimmten Betriebssystemen oder Gerätetypen kompatibel ist. Die Anbieter Readfy und Skoobe stellen keine Softwarelösung für E-Reader ohne Betriebssystem (z. B. Tolino und Kindle) bereit und auch keine Browserversion oder Desktop-Version für PC und Laptop.

Die Kategorie ist zu erfassen, wenn Kunden erwähnen, dass sie den Dienst nicht mit ihrem EReader, ihrem PC oder ihrem Laptop nutzen können oder ihr Betriebssystem vom Anbieter nicht unterstützt wird. Eine Codierung erfolgt auch, wenn Kunden eigene Wege gefunden haben, ein Kompatibilitätsproblem zu lösen.

Beispiele: „Und mittlerweile kann ich Skoobe auch auf dem Windows 8.1 Tablet genießen. Habe dazu nur DuOS aus dem Windows App Store installiert. Dies emuliert ein komplettes Android unter Windows. “; „Ich habe bislang immer nur auf meinem Sony Reader Ebooks gelesen. Mittlerweile bin ich jedoch auch Kundin von Skoobe und genieße seitdem viele Ebooks auch über die App für mein AndroidHandy."

Nicht codiert werden Fälle, bei denen es sich um Programmierfehler oder Bedienungsfehler handelt.

0 nicht erwähnt

1 Kompatibilitätsprobleme erwähnt

11 Problem besteht

12 Problem durch Kunden gelöst

V3C: Zugriff auf das Kundenkonto mit mehr als einem Gerät

Bücher werden im Alltag teilweise auf unterschiedlichen Geräten gelesen. In diesem Fall ist es von Bedeutung, dass der Kunde mehrere Geräte in seinem Kundenkonto registrieren kann.

Codiert wird, wenn der Kunde den Dienst auf mindestens zwei Geräten nutzt oder wenn er diese Möglichkeit bewertet. Im diesem Zusammenhang kann es eine Rolle spielen, ob die Software fähig ist, die Daten eines Kundenkontos zwischen verschiedenen Geräten zu synchronisieren, sodass Kunden z. B. die Lektüre auf jedem 
Gerät an derselben Stelle im Buch fortsetzen können. Es wird davon ausgegangen, dass Kunden den Dienst auf mehr als einem Gerät nutzen, wenn sie das Thema Synchronisation erwähnen. Code 11 ist zu verwenden, wenn Kunden die Synchronisation explizit erwähnen und Code 12, wenn der Kunde nur auf die Geräteanzahl eingeht.

0 nicht erwähnt

1 erwähnt

11 Synchronisation erwähnt

12 Synchronisation nicht erwähnt

\section{Dimension 2: Gemeinsame Nutzung des Zugangs}

Hierunter fallen Äußerungen, die deutlich machen, dass Kunden den Dienst gemeinsam mit anderen Personen nutzen z. B. mit Familienmitgliedern oder Partnern. Dies wir als Account-Sharing bzw. Teilen des Kundenkontos bezeichnet.

Beispiel: „Meine Kinder lesen auch Skoobe-Bücher.“

\section{V4B: Teilen des Kundenkontos}

0 nicht erwähnt

1 erwähnt

\section{Dimension 3: Kundenaktivitäten}

Es wird angenommen, dass die Kommentare sich auf Nutzungsepisoden beziehen, sodass sich die Aktivitäten ableiten lassen, denen die Kunden während der Dienstnutzung nachgehen. Teilweise bedingen sich die Tätigkeiten gegenseitig z. B. muss der Kunde ein Buch erst auswählen, um es lesen zu können. Die Auswahl erfolgt entweder durch Suche oder Stöbern im Bestand oder durch die Suche in der eigenen vorselektierten Sammlung (Bücherlisten), was den Auswahlprozess beschleunigen kann. Um Bücher aus einer eigenen Sammlung auswählen zu können, ist es wiederum notwendig, dass der Kunde Listen anlegt. Bei Readfy können Kunden zudem in Bücherlisten von anderen Kunden stöbern.

\section{V5 A: Bücher lesen}

0 nicht erwähnt

1 erwähnt

V5B: Bücher suchen/ im Bestand stöbern

0 nicht erwähnt

1 erwähnt

V5C: Bücherlisten anlegen und Bücher ordnen

0 nicht erwähnt

1 erwähnt 
V5D: Bücherlisten anderer Kunden lesen

0 nicht erwähnt

1 erwähnt

V5E: Buchrezensionen schreiben und lesen

0 nicht erwähnt

1 erwähnt

V5F: Buchbewertungen abgeben und lesen

0 nicht erwähnt

1 erwähnt

V5G: Bücher mit anderen Menschen teilen

z. B. per E-Mail, in sozialen Netzwerken oder Instant Messaging

0 nicht erwähnt

1 erwähnt

V5H: Sich über Neuerscheinungen informieren

0 nicht erwähnt

1 erwähnt

Dimension 4: Individuelle Anpassung (Customization)

Customization beschreibt die Möglichkeit für den Kunden, den Dienst an seine Bedürfnisse und seine Vorstellungen anzupassen. Hierfür muss der Anbieter technische Strukturen schaffen, d. h. Einstellungsoptionen und Funktionen vorgeben. Anpassungsmöglichkeiten können sich für den Kunden im Zusammenhang mit verschiedenen Aktivitäten als sinnvoll erweisen. Codiert werden Äußerungen im Zusammenhang mit der Suche von Büchern bzw. dem Stöbern im Bestand, dem Lesen von Büchern und dem Umgang mit persönlichen Buchsammlungen. Sollte eine Aussage im Sinne der Definition interpretiert werden können, jedoch keine Zuordnung zu den genannten Tätigkeiten möglich sein, ist V6D: Andere zu codieren. Codiert werden auch Möglichkeiten, die noch nicht vorhanden, aber vom Kunden gewünscht werden. Nicht codiert wird hier die Möglichkeit, Daten zwischen Geräten zu synchronisieren.

V6 A: Individuelle Anpassung bei der Suche nach Büchern oder beim Stöbern

z. B. Suchergebnisse nach Kriterien filtern, bestimmte Themenkategorien ausblenden etc.

0 nicht erwähnt

1 erwähnt 
V6B: Individuelle Anpassung beim Lesen von Büchern

z. B. Helligkeit, Hintergrundfarbe, Schriftgrad, Displayausrichtung, Zoom, Lesezeichen setzen, Buchtext vorlesen lassen, Übersetzungen anzeigen lassen, etc.

0 nicht erwähnt

1 erwähnt

V6C: Individuelle Anpassung beim Organisieren von Büchern

z. B. Liste mit Buchtiteln erstellen, Buchtitel als Favoriten markieren, etc.

0 nicht erwähnt

1 erwähnt

V6D: Andere: Erwähnung

0 nein

$1 \mathrm{ja}$

\section{Dimension 5: Personalisierung}

Unter Personalisierung wird eine auf den Kunden angepasste Leistung verstanden, die automatisch, d. h. ohne Zutun des Kunden, erstellt wird. Sie erfolgt durch die Datenverarbeitung eines technischen Systems auf Basis von Daten des Kundenprofils und/ oder mittels Daten über die Nutzungssituation (Ort, Zeit etc.). Sollte eine Aussage im Sinne der Definition interpretiert werden können, jedoch keine Zuordnung zu den genannten Tätigkeiten möglich sein, ist V7D: Andere zu codieren. Codiert werden auch personalisierte Leistungen, die noch nicht vorhanden, aber vom Kunden gewünscht werden.

Beispiel: „Bloß schade, dass beim Öffnen der App nicht gleich die persönliche Bibliothek oder das zuletzt gelesene Buch mitgeöffnet wird"

Nicht codiert wird die Fähigkeit des Dienstes, automatisch Daten zwischen Geräten zu synchronisieren.

\section{V7 A: Personalisierung bei der Suche nach Büchern oder beim Stöbern}

z. B. Anzeige von ähnlichen Büchern

0 nicht erwähnt

1 erwähnt

V7B: Personalisierung beim Lesen der Bücher

z. B. zuletzt gelesene Stelle/ Seite im Buch merken, Anzahl gelesener Seiten anzeigen

0 nicht erwähnt

1 erwähnt

V7C: Personalisierung beim Organisieren von Büchern

z. B. Historie der gelesenen/ geliehenen/ geöffneten Bücher anzeigen etc.

0 nicht erwähnt

1 erwähnt 


\section{V7D: Andere: Erwähnung}

0 nein

$1 \mathrm{ja}$

\section{Dimension 6: Buchbestand}

Die Dienste präsentieren die Bücher als Katalog, in dem die Kunden nach Büchern suchen und stöbern können. Die Kunden können verschiedene Eigenschaften des Katalogs bewerten. Die Begriffe Sortiment, Bestand und Katalog werden synonym aufgefasst.

\section{V8 A: Aktualität der Bücher im Bestand}

Die Kategorie ist zu erfassen, wenn Kunden bewerten, ob Neuerscheinungen im Bestand vorhanden sind und wie schnell diese sich nach Erscheinen bzw. Veröffentlichung im Bestand befinden.

Beispiel: „Die aktuellen Bestseller werden in aller Regel leider zeitverzögert zur Verfügung gestellt"

Anmerkung: Die Fundstelle ist zusätzlich in den Kategorien „Bekanntheit/ Popularität der Bücher im Bestand“ und „Bestandserweiterung“ zu erfassen.

0 nicht erwähnt

1 erwähnt

\section{V8B: Bestandserweiterung}

Diese Kategorie ist zu erfassen, wenn Kunden bewerten, ob der Bestand regelmäßig um neue Bücher erweitert wird oder wenn deutlich wird, dass sie dies erwarten.

Beispiel: „Ich hoffe das Angebot verbessert sich noch“

Anmerkung: Der Aussage lässt sich auch entnehmen, dass der Kunde noch andere Eigenschaften des Bestands (negativ) bewertet. Da diese jedoch nicht konkretisiert werden, können keine anderen Eigenschaften codiert werden, weil nicht klar ist, welche dies sind.

Nicht codiert werden Kommentare, in denen sich der Kunde wünscht, dass bestimmte Titel ins Sortiment aufgenommen werden.

0 nicht erwähnt

1 erwähnt

\section{V8C: Kontinuität des Bestands}

Hier wird codiert, wenn Kunden bewerten, ob Bücher dauerhaft im Bestand verfügbar sind.

Beispiel: „manchmal wird auch ein Buch was ich mir runtergeladen habe aus meiner Liste genommen"

0 nicht erwähnt

1 erwähnt 


\section{V8D: Größe des Bestands}

Diese Kategorie ist zu erfassen, wenn Kunden bewerten, ob eine ausreichende Anzahl an Büchern vorhanden ist, aus denen gewählt werden kann. Dies kann sich auf den Bestand allgemein oder auch bestimmte Genre bzw. Buchkategorien beziehen. Hier wird auch codiert, wenn Kunden die Auswahl an Büchern ohne nähere Erläuterung bewerten.

Beispiel: Gute Auswahl Für viele Leser der richtige Roman vorhanden

0 nicht erwähnt

1 erwähnt

\section{V8E: Bekanntheit/ Popularität der Bücher im Bestand}

Diese Kategorie ist zu erfassen, wenn Kunden bewerten, ob Bestseller oder Titel von bekannten Verlagen und Autoren gelesen werden können.

Beispiel: „entdeckte dann einige aktuelle Topseller, die ich nicht erwartet haette“

Anmerkung: Die Fundstelle ist zusätzlich in den Kategorien „Aktualität der Bücher im Bestand“ zu codieren.

0 nicht erwähnt

1 erwähnt

\section{V8F: Inhaltliche Qualität der Bücher}

Die Kategorie ist zu erfassen, wenn Kunden das Qualitätsniveau der Bücher und des Bestands insgesamt bewerten.

Beispiel: „Die Bücher sind kostenlos und trotzdem leidet die Qualität nicht“

0 nicht erwähnt

1 erwähnt

\section{V8G: Inhaltliche Vielfalt der Bücher im Bestand}

Eine Codierung erfolgt, wenn ein Kunde das Spektrum an Büchern betont, aus dem gewählt werden kann. Im Vordergrund steht also die Unterschiedlichkeit der Titel und Genre, die im Bestand vorhanden sind.

Beispiel: Eine große Auswahl an verschiedenen Büchern.

Anmerkung: Die Fundstelle ist zusätzlich in der Kategorie „Größe des Bestands“ zu erfassen.

0 nicht erwähnt

1 erwähnt

\section{V8H: Fremdsprachige Bücher im Bestand}

In dieser Kategorie wird erfasst, wenn Kunden thematisieren, ob fremdsprachige Titel im Bestand vorhanden sind bzw. deutlich wird, dass sie dies erwarten. Als 
fremdsprachig gelten Bücher, die nicht auf Deutsch verfasst wurden.

0 nicht erwähnt

1 erwähnt

V8I: Vollständigkeit von Serien und Reihen

Codiert wird, wenn Kunden bewerten, ob Buchserien bzw. Reihen vollständig im Bestand vorhanden sind. Eine Codierung erfolgt nicht, wenn Kunden nur erwähnen, dass sie Serien lesen.

0 nicht erwähnt

1 erwähnt

\section{V8J: Übereinstimmung mit unspezifischen Präferenzen}

In dieser Kategorie wird erfasst, wenn Kunden bewerten, ob sie Titel im Bestand finden, die ihren persönlichen Vorlieben oder Interessen entsprechen. Die Aussage verbleibt auf einer allgemeinen Ebene, d. h. es werden keine Autoren o. ä. erwähnt. Das Urteil kann positiv, negativ oder nicht eindeutig ausfallen.

0 nicht erwähnt

1 Urteil insgesamt positiv

2 Urteil insgesamt negativ

3 Urteil unklar

V8K: Übereinstimmung mit spezifischen Präferenzen

Hier werden Aussagen codiert, in denen Kunden konkrete Angaben dazu machen, was sie sich vorstellen bzw. was sie lesen möchten, und wenn sie bewerten, ob der Bestand diese Vorgaben erfüllen kann. Das Urteil kann positiv, negativ oder nicht eindeutig ausfallen.

Beispiel: „leider noch keine meiner Lieblingsautoren“

0 nicht erwähnt

1 Urteil insgesamt positiv

2 Urteil insgesamt negativ

3 Urteil unklar

\section{V8L: Strukturierung des Buchbestands}

In dieser Kategorie sind Aussagen zu erfassen, die sich mit der Orientierung im Buchbestand beschäftigen. Kunden bewerten, ob sie eine Ordnung im Hinblick auf die Einteilung der Genres erkennen, ob diese Ordnung sich mit ihren Vorstellungen deckt und ob die Stärke der Untergliederung des Buchbestands ausreichend ist z. B. die Anzahl an Kategorien.

Beispiel: „Ich würde Kleinkind und Jugenbücher trennen, da es nervt Jungendliebesromane zwischen ,Klein Olli lernt schreiben'- Büchern zu finden."

0 nicht erwähnt

1 Urteil insgesamt positiv 
2 Urteil insgesamt negativ

3 Urteil unklar

\section{Dimension 7: Finanzielle Kosten der Dienstnutzung}

Je nach Erlösmodell des Anbieters verursacht die Dienstnutzung finanzielle Kosten für den Kunden oder nicht. Readfy war bis März 2016 ausschließlich werbefinanziert und kann immer noch kostenlos genutzt werden. Für Skoobe ist hingegen ein monatlicher Betrag zu entrichten.

\section{V9A: Kosten: keine Erwähnung}

Diese Kategorie ist zu erfassen, wenn Kunden sich in ihrem Kommentar nicht mit den finanziellen Kosten der Dienstnutzung auseinandersetzen.

0 nein

$1 \mathrm{ja}$

\section{Sparpotenzial}

Diese Kategorie ist zu erfassen, wenn Kunden die Kosten für die Dienstnutzung mit den Kosten vergleichen, die bei anderen Beschaffungsformen anfallen und abwägen, ob ihnen der Dienst hilft, weniger Geld für das Lesen von Büchern auszugeben. Das so gebildete Vergleichsurteil kann positiv für den Dienst, negativ für den Dienst oder nicht eindeutig ausfallen.

Beispiel: „Da vor allen Dingen Neuerscheinungen, die erst nur im Hardcover vorliegen als eBook vollkommen überteuert sind, ist dies eine tolle Alternative."

Nicht codiert werden Aussagen, in denen der Dienst nur als kostenlos oder günstig bewertet wird, da hier die direkte Vergleichskomponente fehlt.

\section{V9B: Sparpotenzial im Vergleich zum Kauf von Büchern}

0 nicht erwähnt

1 Urteil insgesamt positiv

2 Urteil insgesamt negativ

3 Urteil unklar

V9C: Sparpotenzial im Vergleich zur öffentlichen Bibliothek

0 nicht erwähnt

1 Urteil insgesamt positiv

2 Urteil insgesamt negativ

3 Urteil unklar

V9D: Andere: Erwähnung

0 nicht erwähnt

1 Urteil insgesamt positiv

2 Urteil insgesamt negativ

3 Urteil unklar 


\section{V9E: Keine/ niedrige Kosten als Trostpflaster}

Diese Kategorie ist zu erfassen, wenn Kunden einen Zusammenhang herstellen zwischen subjektiv niedrigen Kosten und ihrer Beobachtung, dass bestimmte Leistungserwartungen nicht erfüllt werden.

Beispiel: „Ist klar das es nicht alle Bücher gibt...die App ist ja auch kostenlos.“

0 nicht erwähnt

1 erwähnt

\section{V9F: Kosten-Buchlesemengen-Verhältnis}

Diese Kategorie ist zu erfassen, wenn Kunden bewerten, ob sich die Dienstnutzung finanziell lohnt und dieses Urteil davon abhängig machen, wie viele Bücher gelesen werden. Eine Codierung erfolgt auch dann, wenn der finanzielle Vorteil nur indirekt zum Ausdruck kommt.

Beispiel: „Es gibt leider nicht alle Bücher die ich gern lesen würde. Lohnt sich für mich aber trotzdem, da ich viel lese. “

Nicht codiert wird, wenn Kunden lediglich bewerten, für welchen Lesertyp sich der Dienst ihrer Meinung nach eignet.

Beispiel: „Für Vielleser echt super!“

Anmerkung: Diese Aussage ist in der Kategorie V12 A: Lesertyp zu codieren.

0 nicht erwähnt

1 erwähnt

\section{V9G: Finanzierung}

Codiert wird, wenn Kunden einen Zusammenhang herstellen zwischen finanziellen Kosten bzw. Werbeeinblendungen und ihrer Notwendigkeit, damit sich der Dienst finanzieren kann bzw. Autoren und Verlage für die Veröffentlichung der Bücher finanziell entlohnt werden können.

Beispiel: „zumal sie (die Werbung; Anm.) ja das kostenlose lesen ausmacht.“

0 nicht erwähnt

1 erwähnt

\section{Dimension 8: Werbung}

Bei werbefinanzierten Diensten kommen die Kunden beim Bücherlesen mit Werbemitteln in Kontakt. In diesem Fall nehmen die Leser die Werbung zwangsläufig wahr, da das Werbemittel parallel zum Buchinhalt angezeigt wird. Bei Werbung, die sich über den Buchinhalt legt, können sich die Kunden der Werbung nur entziehen, indem sie wegschauen (Pop-Up-Fenster). Bei einer Überlagerung müssen Kunden das Werbemittel aktiv schließen, um weiterlesen zu können oder warten, bis es sich von alleine schließt. Bei Bannerwerbung am Seitenrand können Kunden versuchen, das Werbemittel zu ignorieren. Kunden bewerten, ob sie sich von Werbeeinblen- 
dungen gestört fühlen oder nicht. In diesem Zusammenhang können Kunden versuchen, sich beim Lesen stärker auf den Buchinhalt zu konzentrieren bzw. die Umgebung auszublenden. Dieses Verhalten wird als Werbevermeidungsstrategie aufgefasst.

Beispiel:" beim lesen setzt mein Kopfkino ein und dann stört auch sowas nicht mehr,"

Kunden können zudem statisch-visuelle Werbung am Seitenrand und Werbemittel unterscheiden, welche die Aufmerksamkeit stärker auf sich ziehen, weil sie animiert sind, akustische Reize einbinden oder den Buchtext überlagern (reizstarke Werbung).

\section{V10 A: Werbung stört beim Lesen von Büchern}

Diese Kategorie wird erfasst, wenn Kunden die Werbung beim Lesen als störend bewerten. Codiert wird neben einer eindeutigen Meinung auch eine Tendenz, d. h. die Ausprägung 1 soll auch dann codiert werden, wenn der Kunde die eigentliche Aussage verhüllt. Zum Beispiel kann die Aussage „stört nicht so sehr“ als „,stört, aber nicht so sehr" aufgefasst werden. Ebenfalls soll

Code 1 verwendet werden, wenn der Kunde eine negative Bewertung von Werbung mithilfe von Verbindungswörtern wie trotz, obwohl, obgleich, aber, jedoch oder allerdings zum Ausdruck bringt.

Beispiel: "Gefällt mir Trotz Werbung super"

Auch wird codiert, wenn der Kunde angibt, die Werbung störe nicht, weil sonst eine andere Leistung wegfiele, die ihm wichtig ist z. B. dass das Angebot kostenlos bereitgestellt wird.

Beispiel: „,ie Werbung, mich nervt sie nicht, weil wenn keine Werbung, dann auch keine kostenlose Bücher zum Lesen."

0 nicht erwähnt

1 erwähnt

11 reizstarke Werbung erwähnt

12 Werbungvermeidungsstrategie erwähnt

13 reizstarke Werbung und Werbevermeidungsstrategie erwähnt

14 reizstarke Werbung und Werbevermeidungsstrategie nicht erwähnt

V10B: Werbung stört nicht beim Lesen von Büchern

Diese Kategorie wird erfasst, wenn Kunden die Werbung beim Lesen als nicht störend bewerten.

0 nicht erwähnt

1 erwähnt

11 reizstarke Werbung erwähnt

12 Werbungvermeidungsstrategie erwähnt

13 reizstarke Werbung und Werbevermeidungsstrategie erwähnt

14 reizstarke Werbung und Werbevermeidungsstrategie nicht erwähnt 


\section{Dimension 9: Vergleichsurteile}

Kunden bewerten die Eigenschaften des Angebots auf der Grundlage ihrer bisherigen Erfahrungen im Leben und wägen ab, welche Vorteile und Nachteile sich durch die Dienstnutzung ergeben. Als Vergleichsgröße interessieren in dieser Arbeit der Kauf von Büchern und die Leihe in einer Bibliothek (gedruckt oder digital). Codiert wird auch, wenn Kunden bewerten, was sich im Vergleich zum Umgang mit gedruckten Büchern ändert.

Beispiel: „Auch für den Urlaub platzsparend (die App; Anm.)“

Nicht codiert wird, wenn Kunden nur die finanziellen Kosten als Vergleichswert nutzen, sondern Kostenvergleiche sind in der Kategorie „Sparpotenzial“ zu codieren.

V11 A: Vergleich mit gedruckten Büchern

0 nicht erwähnt

1 erwähnt

V11B: Vergleich mit öffentlichen Bibliotheken

0 nicht erwähnt

1 erwähnt

V11C: Vergleich mit Kauf von Büchern im Handel

0 nicht erwähnt

1 erwähnt

V11D: Andere: Erwähnung

0 nein

$1 \mathrm{ja}$

V11E: Leseintensität im Vergleich zu anderen (Lesertyp)

Hier werden Äußerungen codiert, in denen Kunden sich selbst oder andere die Eigenschaft zuschreiben, viel oder wenig zu lesen.

Beispiel: „Echt toll für Leseratten“

0 nicht erwähnt

1 erwähnt

\section{V11F: Rückkehr zum Lesen}

Hier ist zu codieren, wenn Kunden erwähnen, dass sie mit dem Dienst angefangen haben überhaupt Bücher zu lesen oder wieder regelmäßig Bücher zu lesen. Nicht codiert wird, wenn Kunden sich generell als Menschen beschreiben, die schnell lesen oder viele Bücher lesen bzw. häufig lesen. Auch nicht codiert wird, wenn Kunden angeben mit dem Angebot viel zu lesen.

0 nicht erwähnt

1 erwähnt 


\section{Dimension 10: Freiheit beim Zugang und der Nutzung}

Kunden bewerten, an welche Bedingungen der Gebrauch eines Dienstes geknüpft ist und welche Einschränkungen sich ergeben. Die Bewertung kann sich auf ganz unterschiedliche Aspekte des Dienstes beziehen. Hier soll gemessen werden, wie frei sich die Kunden beim Lesen der Bücher fühlen. Folgende Fragen werden als Indikatoren aufgefasst:

- Kann ich so viel lesen wie ich möchte oder gibt es Einschränkungen?

- Kann ich so lange lesen wie ich möchte oder gibt es Einschränkungen?

- Kann ich lesen bzw. anfangen zu lesen wann ich möchte oder gibt es Einschränkungen?

- Fühle ich mich gehemmt, die Lektüre abzubrechen, wenn mir ein Buch nicht völlig zusagt?

Beispiel: „wenn es nicht mein Geschmack ist, gebe ich es einfach zurück“

Die Urteile können positiv, negativ oder nicht eindeutig ausfallen. Darüber hinaus können Kunden erwähnen, dass sie sich freier und unbefangener fühlen, wenn sie bei der Nutzung nicht über die Kosten nachdenken müssen. Begünstigt wird die Entstehung des Gefühls durch die Abrechnungsmethode Flatrate (Skoobe) und fehlende Kosten (Readfy).

Beispiel: „So braucht man auf tolle Bücher nicht verzichten, auch wenn man nicht viel Geld ausgeben kann oder will“"

Nicht codiert wird, wenn Kunden erwähnen, dass sie den Dienst als günstig empfinden. Demgegenüber können Kunden darauf eingehen, dass sie finanzielle Ausgaben für den Dienst tätigen, um sich zu motivieren mehr Bücher zu lesen. Als Vergleichsbeispiel kann die Anmeldung in einem Fitnessstudio mit Pauschaltarif genannt werden. Der Grund für die Anmeldung ist die Absicht des Kunden mehr Sport zu treiben. Der Kunde setzt sich durch die pauschale Bezahlung bewusst dem Risiko aus, dass sich der finanzielle Aufwand für ihn nicht lohnt, wenn er den Dienst zu wenig in Anspruch nimmt z. B. wenig liest oder das Fitnessstudio nur selten besucht. Der Kostendruck dient dem Kunden also als Hilfsmittel, um in einer Entscheidungssituation die eigenen Handlungsoptionen einzuschränken und wird (zunächst) nicht negativ bewertet.

\section{V12 A: Freiheit bei der Buchlesemenge}

0 nicht erwähnt

1 Urteil insgesamt positiv

2 Urteil insgesamt negativ

3 Urteil unklar

V12B: Freiheit beim Lesezeitraum

0 nicht erwähnt 
1 Urteil insgesamt positiv

2 Urteil insgesamt negativ

3 Urteil unklar

V12C: Freiheit beim Lesebeginn

Eine Codierung erfolgt, wenn Kunden bewerten, ob sie auf einen Titel, der sich im Bestand befindet, sofort zugreifen können oder ob der Zugriff von Bedingungen abhängt, welche den Lesebeginn verzögern oder verhindern. Das Urteil kann positiv, negativ oder nicht eindeutig ausfallen.

Beispiel: „man kann sofort anfangen das ausgesuchte buch zu lesen ohne wartezeit“

0 nicht erwähnt

1 Urteil insgesamt positiv

2 Urteil insgesamt negativ

3 Urteil unklar

V12D: Freiheit Lektüre abzubrechen wegen Nichtgefallen

0 nicht erwähnt

1 Urteil insgesamt positiv

2 Urteil insgesamt negativ

3 Urteil unklar

V12E: Entkopplung von Kosten und Lektüre

0 nicht erwähnt

1 erwähnt

V12F: Selbstdisziplinierung durch finanzielle Ausgaben

0 nicht erwähnt

1 erwähnt

\section{Dimension 11: Leichtigkeit der Benutzung}

Kunden investierten Zeit und Mühe, wenn sie den Dienst nutzen bzw. um ihre Ziele $\mathrm{zu}$ erreichen. Die Mühe setzt sich aus physischen, emotionalen und kognitiven Kosten zusammen. Kunde erleben es i. d. R. als anstrengend, wenn der kognitive Aufwand hoch ist, weil sie sich viele Gedanken machen müssen. Es wird daher angenommen, dass Kunden bewerten, mit welchem Aufwand die Nutzung für sie verbunden ist und wie leicht ihnen die Nutzung insgesamt fällt. Die Bewertung der Leichtigkeit kann sich auf ganz unterschiedliche Aspekte des Dienstes beziehen.

\section{V13: Leichtigkeit der Benutzung}

Hier soll gemessen werden, wenn Kunden Bezug auf die Orientierungsfreundlichkeit und die Navigierbarkeit nehmen. Zudem soll die Kategorie codiert werden, wenn Kunden bewerten, ob die Benutzung des Angebots leicht zu erlernen ist. 
- Deuten Kunden an, dass sie sich schnell zurechtfinden oder fühlen sie sich eher verloren?

- Erkennen Kunden eine Ordnung und Struktur, die ihnen die Orientierung und Navigation erleichtert?

- Finden Kunden schnell, was sie suchen z. B. ein bestimmtes Buch, eine Einstellungsmöglichkeit oder einen bestimmten Bereich in der App?

- Fällt es Kunden leicht, die Funktionen und den Umgang mit der App zu lernen?

Das Urteil zur Bedienungsfreundlichkeit kann positiv, negativ oder nicht eindeutig ausfallen.

Folgende Adjektive geben Hinweis auf die Bewertung der Einfachheit bei der Benutzung:

- Synonyme für einfach laut Duden: bequem, eingängig, leicht [verständlich], mit Leichtigkeit, mühelos, ohne Anstrengung/Mühe, ohne Schwierigkeit[en], problemlos, simpel, spielend, unangestrengt, unkompliziert, unproblematisch, unschwer; kinderleicht; intuitiv

- Synonyme für kompliziert laut Duden als Gegenteil von einfach: heikel, knifflig, kritisch, mühsam, problematisch, schwer, schwierig, auch: anstrengend und aufwändig

Nicht codiert werden Kommentare, die sich mit Bedienungsproblemen infolge von Programmierfehlern und technischen Störungen beschäftigen.

0 nicht erwähnt

1 Urteil insgesamt positiv

2 Urteil insgesamt negativ

3 Urteil unklar 


\section{Sachregister}

\section{A}

Abbrecher 22 f., 110

Abrufprinzip 32 - 34, 41, 81, 93

Affordance 58 f., 80

Aktivitäten 11, 13, 19, 33, 49 f., 53, 55 - 57, 61 - 63, 72 - 79, 82, 88, 96 - 98, 101, 106, $108-110,144 \mathrm{f}$.

Aktivitätsgrad siehe Aktivitätsniveau 57 Aktivitätsniveau 50

Aktualität 32, 87, 89 f., 147 f.

Algorithmus 69, 73

Analogie-Suche 65, 69, 109

Anbieterintegration 56

Aneignung 50 f., 53, 108

Angebotsformen 11 f., 15 f., 25, 92, 100, $106-108,110$

Angebotsgestaltung 12, 25, 65, 105

Anspruchshaltung siehe Anspruchsniveau 90

Anspruchsniveau 31 f., 82, 91 f., 94, 105

Aufwand (bei der Angebotsnutzung) 28, 33, 44, 46, 49 f., 55, 66, 70, 78, 154 f.

Auswahl (von Büchern) 25, 28, 31, 62, 65 f., 72, 75, 84, 92, 108, 110, 137, 140, 144, 148

Autor 41, 57, 65 f., 68 - 70, 77, 91, 95, 105, 148 f., 151

\section{B}

Banner 44 f., 81, 95

Bedürfnis 49

Beliebtheit (eines Buches) siehe Popularität 59

Benutzungsoberfläche 34, 39, 57 f., 77, 79

Benutzungsschnittstelle 39, 43 f., 58, 81, 89, 94 f., 107

Bereitstellungsdauer 26

Bereitstellungsmodi $32 \mathrm{f}$.
Bereitstellungsqualität 12, 25 - 27,

29 - 34, 36, 38 f., 41, 43 f., 46 f., 88, 93, 107

Bereitstellungsraum 33

Bereitstellungsverfahren (bei Online-

Angeboten) 34, 81, 107

Beschränkungen (bei der Angebotsnutzung) 40 f., 69, 77, 81, 93, 105

Bestand (an Büchern) 22, 30 - 32, 67, 72, 81, 85, 87 - 91, 98, 101, 105, 144 f.,

147 - 149, 155

Bestätigung (von Erwartungen) 31, 57, 83, 105, 137

Bestseller siehe Popularität 32, 92

Besuchsprinzip $32-34$

Betroffenheit 21, 79

Bewertung (von Büchern) 46, 57, 84, 95, 97, 99, 102, 108 f., 137, 139, 141, 152, $154-156$

Beziehung (zwischen Kunden) 50, 53, $57 \mathrm{f}$.

Bibliothek $16 \mathrm{f} ., 32 \mathrm{f} ., 40,42,46,61,67$, 70, 72 f., 79, 86 f., 90, 92 f., 99, 101 f., 106 f., 146, 150, 153

Bring-Prinzip 32 - 34

Browser 35, 81, 107, 142

Buchart 29 f., 41, 67, 87, 89 f., 99, 101, 105, 107, 109

Buchhandel 91

Buchhandlung 25, 28, 30 - 32, 70 f., 74, 93, 106, 109

Buchlesemenge 41 f., 82, 87 f., 101, 106, 151, 154

Buchwissenschaft 11, 13, 26

C

CDL 56, 97

Client-Server-Prinzip 34

Cloud 16, 35, 38, 81, 107

Convenience 47, 54 
Customization 55 - 57, 64 f., 67, 73 f., 76, 78, 82, 87, 98 f., 109, 145

\section{D}

Darstellung (von E-Books) 39, 64, 88, 142

Datei 16, 35, 37, 84, 137

Daten 21, 34 f., 39, 55, 70, 83, 85 f., 102, 108, 139, 141, 143, $145 \mathrm{f}$.

Distanzprinzip 32

Domizilprinzip 32

Download 35, 81, 107, 141

Durchschnittsleser 20 f., 29, 106

\section{E}

ease of use 46

Effizienz 54

Einschränkungen (bei der Angebotsnutzung) siehe Beschränkung 33, 101

Eliminationsphase 66, 92

Empfehlung 67, 69, 74, 78, 105,

$108-110,141$

Empfehlungssystem 69

Entwicklungspsychologie 50

Erlöse (aus Anbietersicht) 39 f., 46, 110

Erscheinungsweise 26, 30

Evaluation 65 f., 68, 80

\section{$F$}

Faktual $29 \mathrm{f}$.

Fehlentscheidung 31, 45 f., 94

Fernsehen 22, 46

Fiktional $29 \mathrm{f}$.

Film 16, 42, 66, 92

Filter 75, 98, 110, 141, 145

Finanzierung 85, 87, 94, 107, 151

Flatrate 40 - 42, 46, 81, 94, 106 f., 154

Flatrate-Bias 42, 94

Fremdsprachige (Bücher) 32, 88 - 90, 105, 148

Funktionen (bei der Angebotsnutzung) 13, 33, 37, 39, 44, 57 - 59, 63 f., 97, 99, 106, 108 -110, 141, 145, 156
G

Gegenstandsbeziehung siehe Gegenstandsbezug

Gegenstandsbezug 51

Genre 29 f., 66, 69, 90 f., 100, 148 f.

Gerät $33-38,58,64,82,84,86,89,100$, 107, $141-146$

\section{$\mathrm{H}$}

Handlung 16, 39, 51 - 53, 55, 58 f., 61, 64, 69, 72, 75, 97

Historie 146

I

Identität 20 f., 79

Ikea-Effekt 57

Individualisierung $63 \mathrm{f}$.

Informationsmanagement 63, 71, $74 \mathrm{f}$.

Inhaltsanalyse 13, 27, 81, 83, 103, 137

Interesse 12, 19, 22 f., 52, 58, 61, 71, 149

Interstitial 81

Involvement 43, 73, 96, 107

$\mathrm{K}$

Kauf (als Angebotsform) 11 f., 15 f., 40 - 42, 49, 61, 66, 86 f., 91, 94, 100, 102, 106 f., 110, 150, 153

Käuferreichweite 15

Kommunikationswissenschaft 50

Kompatibilität 38, 86, 89, 107

Konto (bei einem Anbieter) 40, 82, 106

Kontrolle (des eigenen Verhaltens) 51, 54,76

Kosten (aus Kundensicht) 11, 27, 39 - 46, 49, $54-56,82,85,87-90,94,101$, $105-107,150$ f., $153-155$

Kulturhistorische Schule 50

L

Laden 47, 61 f., 67, 70 f., 100 f., 109

Leichtigkeit der Benutzung 26, 46 f., 88, 155

Leihbücherei 17 
Leihe (als Angebotsform) $11 \mathrm{f}$, 15 -18, 40 f., 87, 90, 102, 106 f., 153

Lernen 54, 156

Lesehäufigkeit 21

Leseintensität 20, 23, 42, 86, 101 f., 106, 153

Leseliste siehe Merkliste 69 f., 72, 75 f., 82, 97 ff., 110

Lesen (als Tätigkeit) 12, 15 f., 19 - 24, 30, 35 f., 38 f., 41 f., 44 f., 51, 58, $61-66,68$, 71 - 74, 78 f., 81 f., 86 f., 89 - 91, 93 - 98, 100 f., 105 - 110, 141 f., 144 - 146, $149-155$

Lesertyp 20, 22, 29, 86 f., 100, 151, 153

Lesestrategie 64, 73, 78, 99

Lesezeichen 64, 72 f., 98, 100, 110, 146

Link 28, 78

Lizenz 92

LpA 41

\section{M}

Merkliste 71 f., 76 - 78, 96, 98, 106, 109

Miete (als Angebotsform) 12, 15, $17 \mathrm{f}$., 42, 81, 107

Mikrostrategien (bei der Buchsuche) $65 \mathrm{f}$.

Motiv 16, 51 f., 57

Mühe 28, 45, 49, 54, 97, $155 \mathrm{f}$.

Musik 11, 54, 63, 69, 71 f., 74, 76, 78

\section{N}

Navigation 59, 68, 74, 156

Netzverbindung 35, 41, 85, 93, $141 \mathrm{f}$.

Neuerscheinung siehe Novität 74, 92, 97, 105

Nichtleser 20

Nordische Schule 56

Notiz(-funktion) 98

Novität 32, 74, 92

Nutzungsintensität 17, 40 f., 43, 57

0

Offline-Lesen 35 f., 81, 85 f., 89, 93, 141 f. Öffnungszeiten 33
Online-Katalog 28 f., 68, 79 f.

Online-Lesen 35, 81

Operationen 39, 55

Opfer 41, 49, 94

Optimierungspotenzial 55, 64, 73, 82, 88, 97 f., 109

Ordnung (von Büchern) 26 f., 29, 67, 85, 88, 90, 107, 149, 156

Organisation (von Informationen und Büchern) siehe Informationsmanagement 63

Orientierung 97, 149, 156

$\mathbf{P}$

Personalisierung 54 - 56, 64 f., 69, 73, 82, 87, 97, 99 f., 109, 146

Popularität (von Büchern) 32, 87, 89 f., $147 \mathrm{f}$.

Pop-Up-Werbung 44 f., 95

Präferenz 27, 31 f., 67, 72, 85, 87, 89, 91, 94, 99, 105, 109 f., 149

Präsentation (von Büchern) 12, 35, $67 \mathrm{f}$.

Precommitmenteffekt 43

Preisvorteil siehe Sparpotenzial 40

Produktivität $54 \mathrm{f}$.

Profil (bei einem Anbieter) 73, $107 \mathrm{f}$.

Programmierung 58

R

Reizarm 44 f., 81 f., 95, 107

Reize 43 - 45, 96, 152

reizstark 44 f., 81 f., 95, 152

Relationship Marketing 49

Reliabilität 85 f., 88

Residenzprinzip 32

Responsiveness 73

Reversibilität 45 f., 105

Rezension 75, 77, 79, 82, 96 f., 108, 139

Reziprozität 74, 76

Risiko 38, 102, 154

Rückgabe 16, 33, 46, 72

Rückkehr 22 f., 101, 153 
$\mathrm{S}$

Sammelobjekt 19, 63

Scanning 65, 67 f., 80, 99, 109

Selbstdisziplinierung 43, 88, 155

Selbstoffenbarung 75 f., 108

Selbstwirksamkeit 75

Sender (Person) 73 f., 76, 78

Serie 30 f., 87, 89 f., 98 f., 149

Situative Umstände 23

Skript $61 \mathrm{f}$.

Software 12, 34 f., 39, 58 f., 63, 142 f.

Sortimentsbildung 27 f., 109

Soziale Erwünschtheit 22

Sparpotenzial 40, 82, 87, 100, 102, 106,

150, 153

Standort 33

Status-Sharing 77

Stöbern 62, 65, 67, 69, 71, 75 f., 78, 80,

96 f., 101, 109, $144-147$

Störung 43 - 45, 82, 95, 156

Strukturierung (von Büchern) siehe

Ordnung 29, 88, 90, 105

subjektive Bedeutsamkeit $21 \mathrm{f}$.

Suchanfrage 65, 68, 70, 80

Suchaufgabe 65, 70, 109

Suchaufwand 28

Suche (von Büchern) 12, 50, 65, 67, $69-72,74,80,82,86$ f., 92, $96-99,102$, 108 f., $144-147,156$

Suchfunktion 59

Suchrichtung 65, 71

Suchstrategie 68, 80, 83, 99, 109

Synchronisierung 86

\section{T}

Tagcloud 70, 79, 109

Tags 69, 79 f., 82, 108

Tarif $40-43,46,81,93,106$

Tätigkeit 19 - 23, 36, 43, 50 - 54, 57, 62 f., 71 f., 79, 97, 101, $144-146$

Tätigkeitstheorie 12, 50 f., 53, 55

Taxametereffekt 42

technisches Format 26

Technologie 86 f., 107
Textsuche 64, 99

Transaktion 40, 106

transaktionsabhängig 40, 46, 81, 106

Transparenz 43 f., 89, 94 f., 107

Transport 33

Treffer 68, 80, 109

Treffprinzip 32

Typografie 64

U

Ubiquität 33, 93, 101, $141 \mathrm{f}$.

Umgebung 28, 47, 50, 53, 55, 57 f., 66, 71, 152

Umwelt 51 - 54, 57

Urteilsbildung (zu einem Angebot) 27, 82, 97, 100

V

Value-in-Use 19, 24, 102

Verbindungen (zwischen Büchern) 62, 68 f., 71, 80, 109

Verfügbarkeit 30 - 32, 82, 87, 89, 91

Vergegenständlichend 54 f., 72, 75, 79

Vergegenständlichung $53 \mathrm{f} ., 72$

Vergleichsprozess 24, 40, 42, 82, 86, 88, 100

Verlag 16 f., 41, 66, 73, 90, 92, 110, 148, 151

Videothek 16, 92

Vielfalt 26 f., 30, 37, 85, 87 - 90, 105, 107, 148

Vielleser 20 f., 30, 71 f., 94, 100 f., 106, 151

Vorauswahl 27, 72

W

Wenigleser 20 f., 41

Werbeeinblendung 45, $94 \mathrm{f}$., $151 \mathrm{f}$.

Werbeeinnahmen $39 \mathrm{f}$.

Werbeform 44, 81, 95, 107

Werbemittel 44 f., 81 f., 95, $151 \mathrm{f}$.

Werbevermeidungsstrategie 45, 82, 95, 152 
Werbung 44 f., 81 f., 87, 89, $94-96,107, \quad$ Z

$151 \mathrm{f}$.

Werbungtreibende 41, 45

Werkzeug 39, 44, 54, 68 f., 80, 108

Wert 19 f., 30, 38 f., 42, 50, 61, 86, 88, 97, 106, 108

Wissen $13,51,54,62,66,100,110$
Zeichen 59

Zeitraum (Buchzugriff) 18, 21, 40 - 42, 85, 110

Ziele 12, 23, 25, 49, 52, 54, 56, 155

Zufriedenheit 31, 137 
